\title{
IDENTIFICATION OF ROTORDYNAMIC FORCES IN A
}

\section{FLEXIBLE ROTOR SYSTEM USING MAGNETIC BEARINGS}

\author{
A Dissertation \\ by \\ ZACHARY SCOTT ZUTAVERN \\ Submitted to the Office of Graduate Studies of \\ Texas A\&M University \\ in partial fulfillment of the requirements for the degree of \\ DOCTOR OF PHILOSOPHY
}

August 2006

Major Subject: Mechanical Engineering 


\title{
IDENTIFICATION OF ROTORDYNAMIC FORCES IN A
}

\section{FLEXIBLE ROTOR SYSTEM USING MAGNETIC BEARINGS}

\author{
A Dissertation \\ by \\ ZACHARY SCOTT ZUTAVERN \\ Submitted to the Office of Graduate Studies of \\ Texas A\&M University \\ in partial fulfillment of the requirements for the degree of \\ DOCTOR OF PHILOSOPHY
}

\begin{abstract}
Approved by:
Chair of Committee, Dara Childs

Committee Members, John Junkins

Alan Palazzolo

Alexander Parlos

Head of Department, Dennis O'Neal
\end{abstract}

August 2006

Major Subject: Mechanical Engineering 


\author{
ABSTRACT \\ Identification of Rotordynamic Forces in a Flexible Rotor \\ System Using Magnetic Bearings. (August 2006) \\ Zachary Scott Zutavern, B.S., Texas A\&M University; \\ B.A., Texas A\&M University; \\ M.S., Texas A\&M University \\ Chair of Advisory Committee: Dr. Dara Childs
}

Methods are presented for parameter identification of an annular gas seal on a flexiblerotor test rig. Dynamic loads are applied by magnetic bearings (MBs) that support the rotor. $\mathrm{MB}$ forces are measured using fiber-optic strain gauges that are bonded to the poles of the MBs. In addition to force and position measurements, a finite element (FE) rotor model is required for the identification algorithms. The FE rotor model matches free-free characteristics of the test rotor. The addition of smooth air seals to the system introduces stiffness and damping terms for identification that are representative of reaction forces in turbomachines. Tests are performed to experimentally determine seal stiffness and damping coefficients for different running speeds and preswirl conditions. Stiffness and damping coefficients are determined using a frequency domain identification method. This method uses an iterative approach to minimize error between theoretical and experimental transfer functions. Several time domain approaches are also considered; however, these approaches do not produce valid identification results. Stiffness coefficients are measured using static test results and an MB current and position based model. Test results produce seal coefficients with low uncertainties for the frequency domain identification method. Static test uncertainties are an order of magnitude larger, and time domain attempts fail to produce seal coefficient measurements. 
In addition to the primary identification research, an investigation of the relationships between MB force, strain, and magnetic field is conducted. The magnetic field of an MB is modeled using commercial FE software. The magnetic field model is used to predict strain measurements for quasi-static test conditions. The strain predictions are compared with experimental strain measurements. Strain predictions agree with experimental measurements, although strain is typically over-predicted. 


\section{DEDICATION}

To my wife and children for their love and support.

To my parents for encouraging me to

challenge myself and pursue my dreams. 


\section{ACKNOWLEDGEMENTS}

I would like to thank Dr. Dara Childs for this exceptional research opportunity and for his support and guidance in my education. It has been an honor to work for a professor who is both a talented researcher and a considerate person. Stephen Phillips and Eddie Denk were also invaluable with their involvement in this project. I appreciate the willingness of Dr. John Junkins, Dr. Alan Palazzolo, and Dr. Alexander Parlos to serve on my dissertation committee. I would also like to thank my fellow graduate students, Adolfo Delgado and Bradley Kerr, for lending me a hand and providing feedback throughout the course of my research. Finally, thanks to the Turbomachinery Research Consortium, whose financial support made this project possible. 


\section{TABLE OF CONTENTS}

\section{Page}

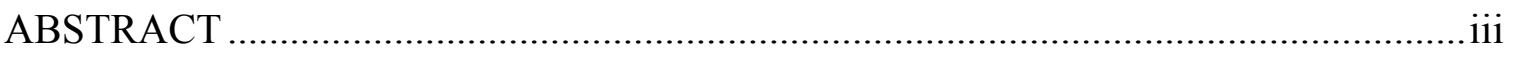

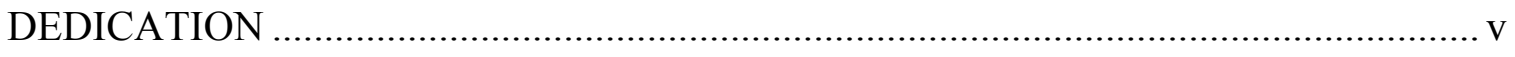

ACKNOWLEDGEMENTS ..............................................................................

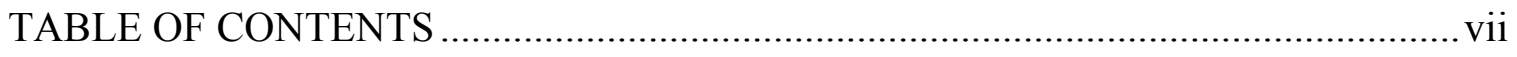

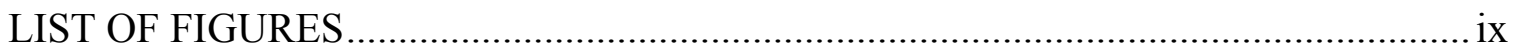

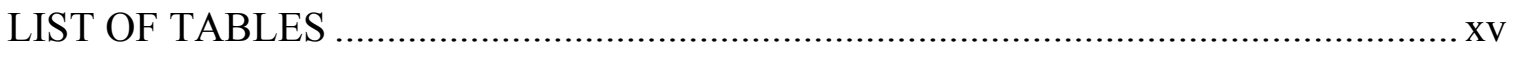

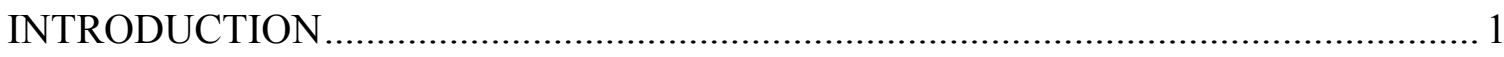

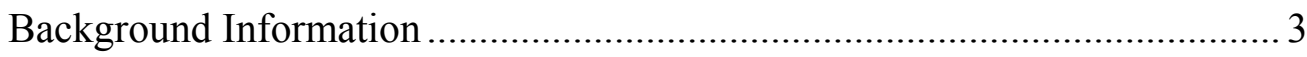

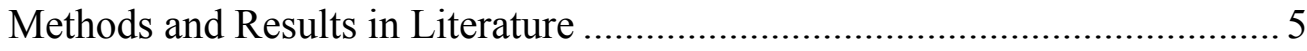

TEST RIG DESCRIPTION AND DESIGN ............................................................ 9

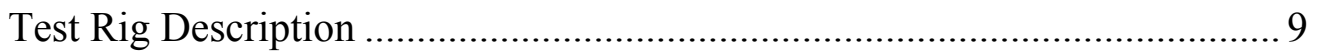

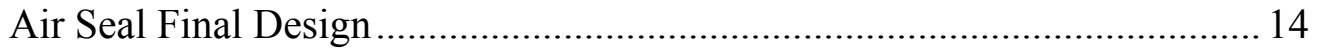

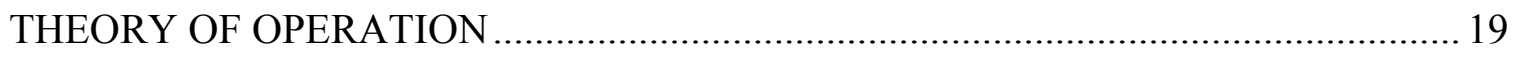

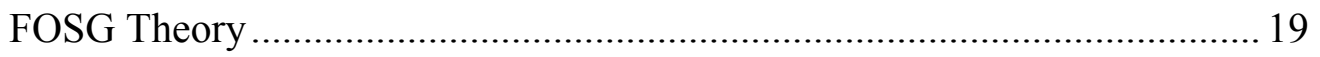

Magnetic Bearing Theory ................................................................. 21

Validation of MB Model for Identification............................................... 23

Dynamic Flexibility Transfer Functions .................................................... 35

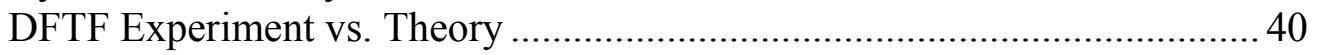

Rotor Model Validation- Rap Test Results and Comparisons ...................... 50

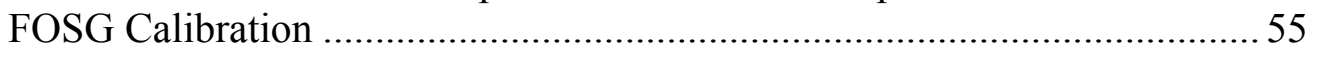

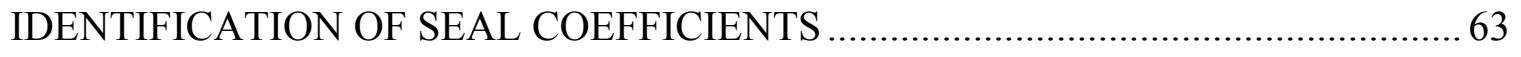

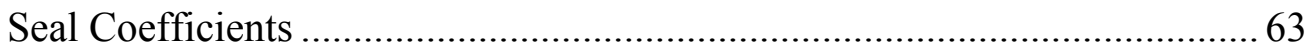

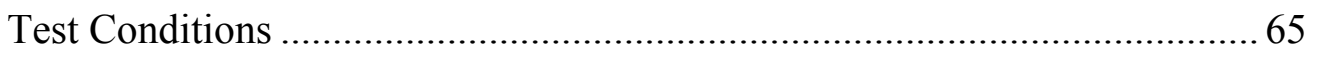

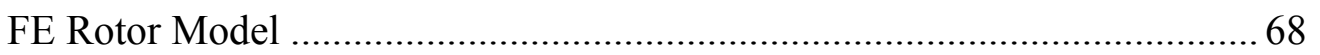

Frequency Domain Identification .................................................... 70

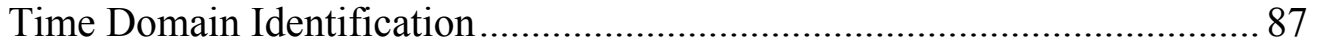




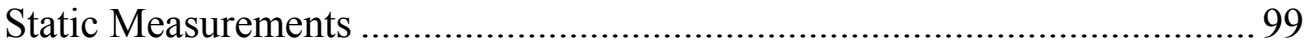

Comparisons with Predictions and Historical Testing .............................. 101

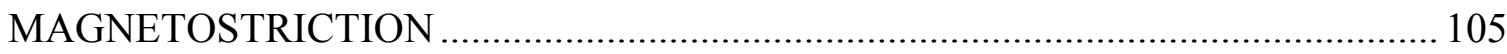

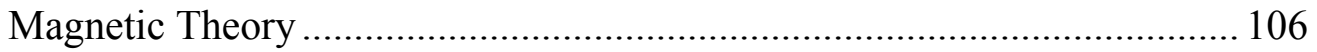

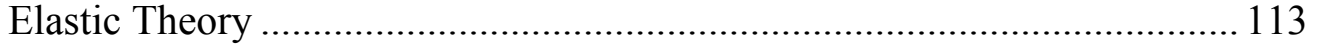

MB Magnetic Field Model................................................................ 114

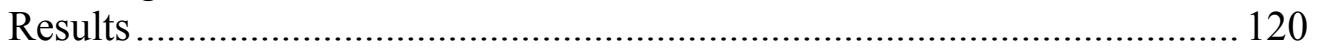

Magnetostriction Summary ......................................................... 125

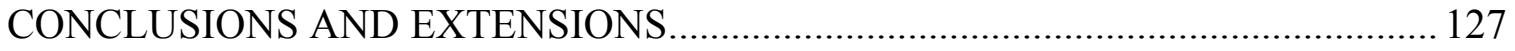

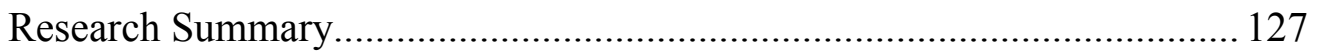

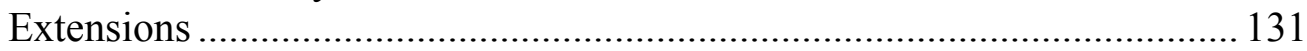

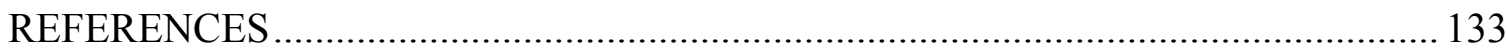

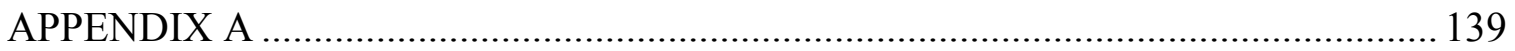

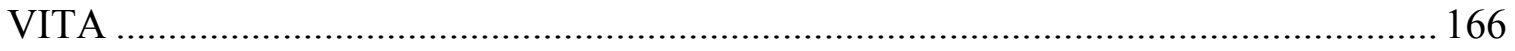




\section{LIST OF FIGURES}

FIGURE

Page

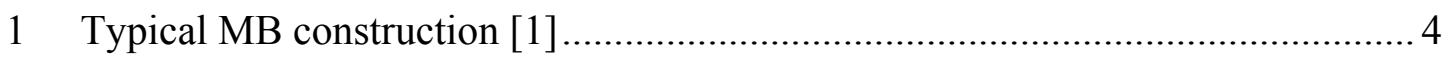

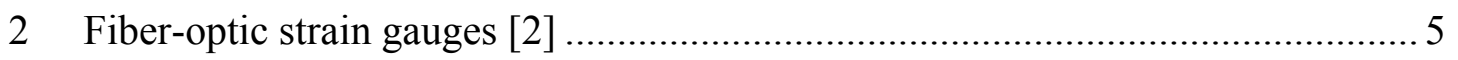

3 Magnetic bearing test rig at TAMU Turbomachinery Laboratory....................... 10

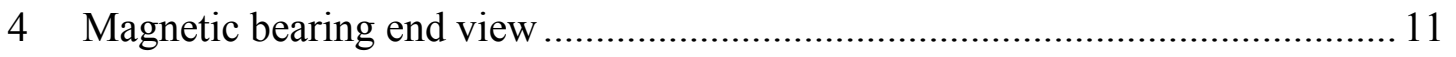

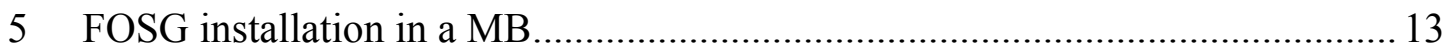

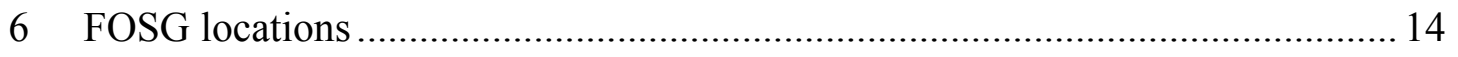

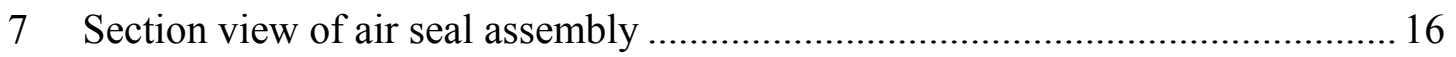

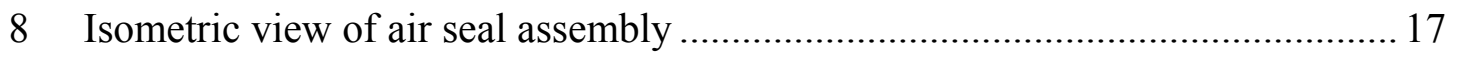

$9 \quad \mathrm{MB}$ test rig with air seal assembly installed................................................... 18

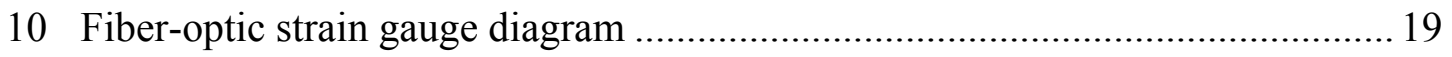

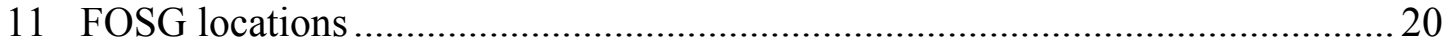

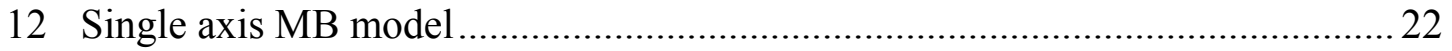

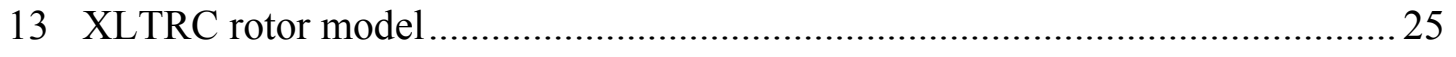

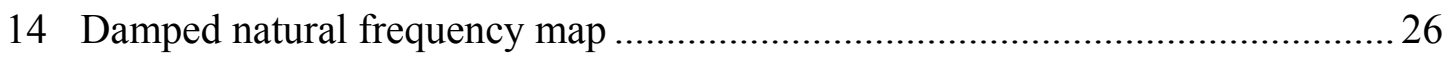

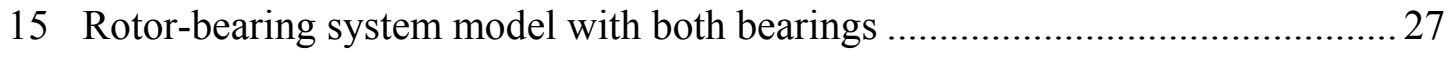

16 Two-bearing response at MB1 to force at MB1 force ..................................... 28

17 Two-bearing response at MB2 to force at MB1 force .................................... 29

18 Two-bearing response at MB1 to force at MB2 force ...................................... 29

19 Two-bearing response at MB2 to force at MB2 force ................................... 30

20 Rotor-bearing system model with drive-end bearing .................................. 31 
FIGURE Page

21 Drive-end response at MB2 to force at MB2 force ........................................ 31

22 Rotor-bearing system model with non-drive bearing...................................... 32

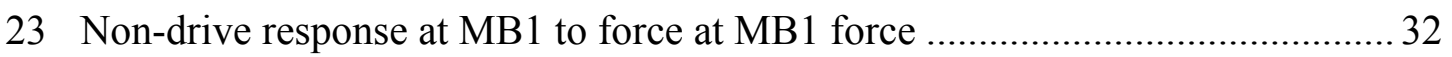

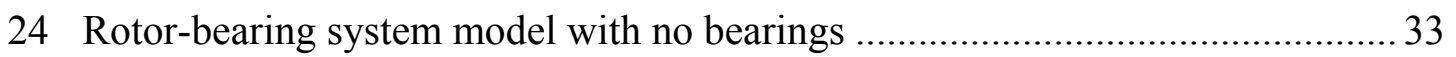

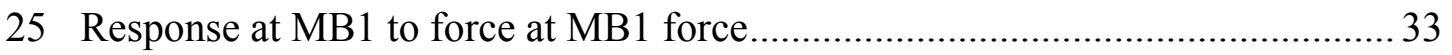

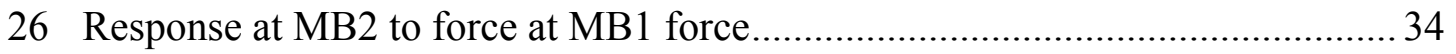

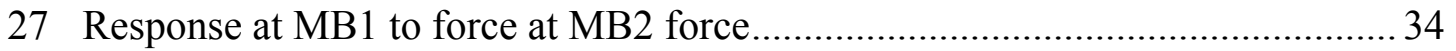

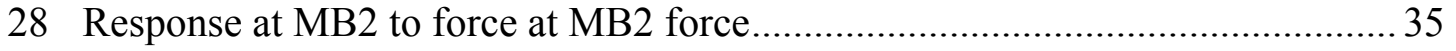

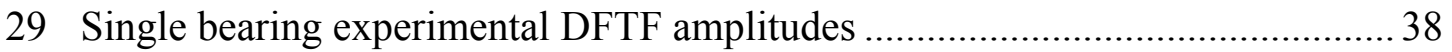

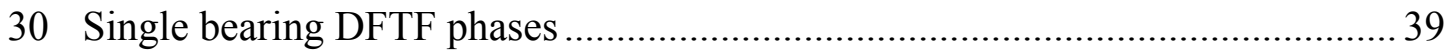

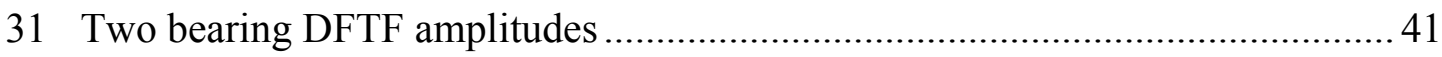

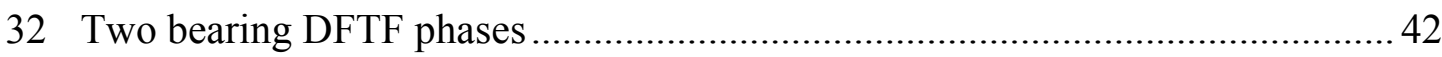

33 Single bearing DFTF comparison for the non-drive bearing

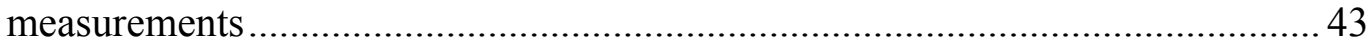

34 Single bearing DFTF comparison for the drive-end bearing

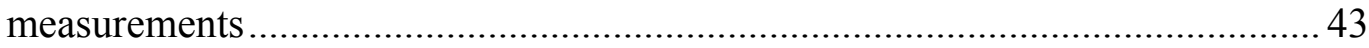

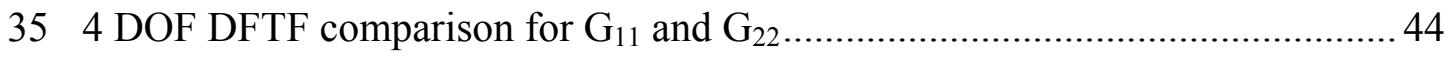

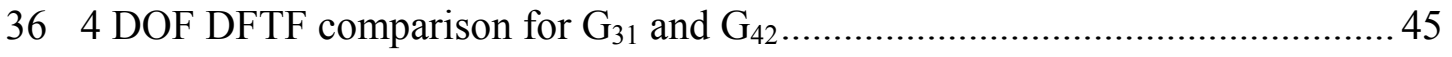

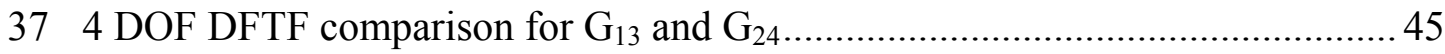

384 DOF DFTF comparison for $\mathrm{G}_{33}$ and $\mathrm{G}_{44 \ldots \ldots \ldots \ldots \ldots \ldots \ldots \ldots \ldots \ldots \ldots \ldots \ldots \ldots \ldots \ldots \ldots \ldots \ldots \ldots \ldots \ldots \ldots \ldots \ldots \ldots \ldots \ldots \ldots \ldots \ldots \ldots \ldots}$

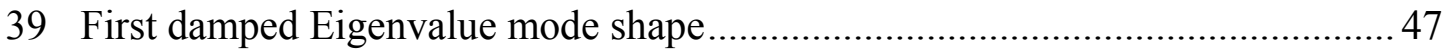

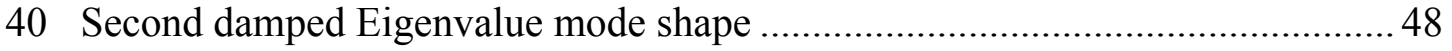

41 Third damped Eigenvalue mode shape ....................................................... 48

42 Model of magnetic bearing as a calibrated exciter.......................................... 49 
FIGURE Page

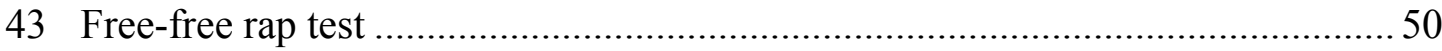

44 Measured free-free rotor modes with coupling ......................................... 51

45 Mode shape comparison between test result and $X L T R C^{2}$ prediction..................52

46 Free-free rotor without coupling mode shapes............................................ 53

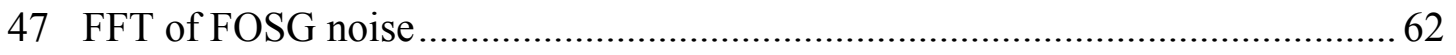

48 Seal pictures- (a) smooth, (b) labyrinth, (c) hole pattern, (d)

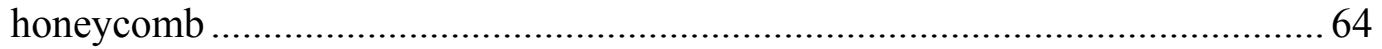

$49 \mathrm{G}_{\mathrm{ij}}($ Eq. (31)) FE model DFTF predictions with and without seal ...................... 68

50 Comparison of experimental and modeled DFTF amplitudes $(\mu \mathrm{m} / \mathrm{N})$

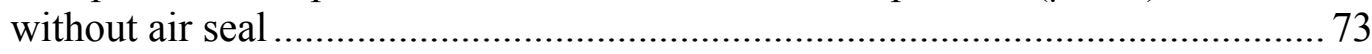

51 Comparison of experimental and modeled DFTF phases $\left(^{\circ}\right)$ without

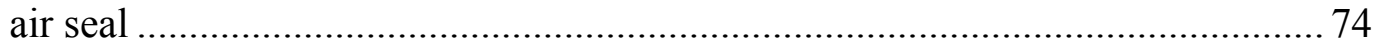

52 Frequency domain identification amplitudes $(\mu \mathrm{m} / \mathrm{N})$ at $0 \mathrm{rpm}$

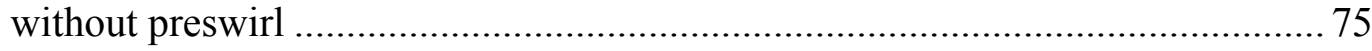

53 Frequency domain identification phases $\left(^{\circ}\right)$ at $0 \mathrm{rpm}$ without

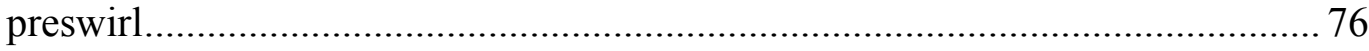

54 Frequency domain identification amplitudes $(\mu \mathrm{m} / \mathrm{N})$ at $7700 \mathrm{rpm}$

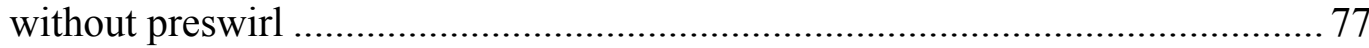

55 Frequency domain identification phases $\left(^{\circ}\right)$ at $7700 \mathrm{rpm}$ without

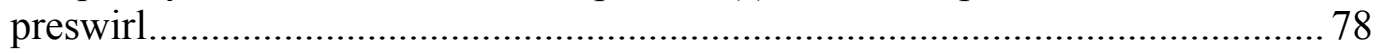

56 Frequency domain identification amplitudes $(\mu \mathrm{m} / \mathrm{N})$ at $0 \mathrm{rpm}$ with

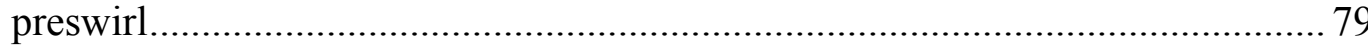

57 Frequency domain identification phases $\left(^{\circ}\right)$ at $0 \mathrm{rpm}$ with preswirl .................... 80

58 Unstable system response to seal with preswirl .......................................... 81

59 Frequency domain identification amplitudes $(\mu \mathrm{m} / \mathrm{N})$ at $7700 \mathrm{rpm}$ with preswirl 
60 Frequency domain identification $\left(^{\circ}\right)$ phases at $7700 \mathrm{rpm}$ with

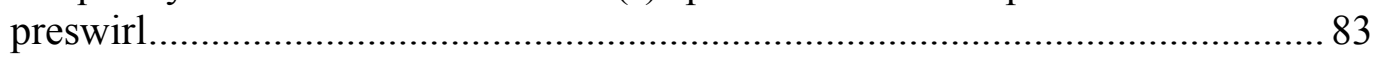

61 Comparison of DFTF amplitudes $(\mu \mathrm{m} / \mathrm{N})$ for two tests with same test conditions 86

62 Comparison of DFTF phases $\left(^{\circ}\right)$ for two tests with same test conditions

63 Comparison of DFTF amplitudes $(\mu \mathrm{m} / \mathrm{N})$ for full and reduced system models

64 Comparison of DFTF phases $\left(^{\circ}\right)$ for full and reduced system models

65 Two degree-of-freedom OKID example 95

66 Comparison of frequency domain test results with XLIsotSl predictions 102

67 Permanent magnet example of compressive forces ........................................ 105

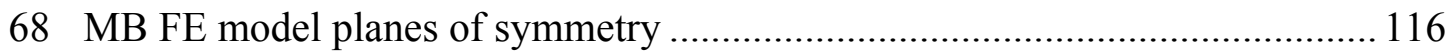

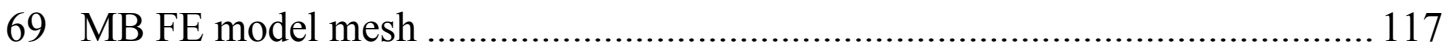

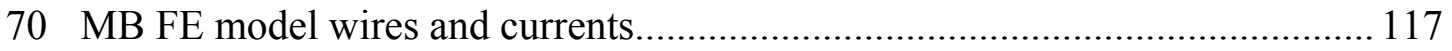

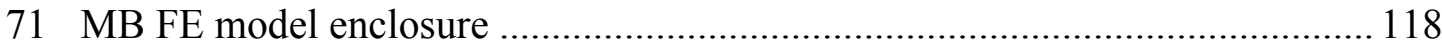

72 MB FE model locations for FOSG measurements........................................ 119

73 B-H curve provide by SKF Magnetic Bearings, Inc...................................... 120

74 MB FE model sample magnetic field..................................................... 121

75 MB FE model sample magnetic field vector plot .................................... 121

76 Non-drive bearing strain test results and FE predictions ............................... 122

77 Non-drive bearing comparison of test results and predictions........................ 123

78 Drive bearing strain test results and FE predictions...................................... 124

79 Non-drive bearing comparison of test results and predictions........................ 125 
FIGURE Page

80 Air flow rates for a single smooth seal with inlet pressure $1.83 \mathrm{MPa}$ (265 psia).

81 Air seal average direct stiffness forces for $10 \mu \mathrm{m}(.39$ mil) shake amplitude

82 Air seal average direct damping forces at $140 \mathrm{~Hz}$

83 Air seal cross-coupled stiffness forces for $10 \mu \mathrm{m}$ (.39 mil) shake amplitude

84 Water flow rates for a single smooth seal with $1.03 \mathrm{MPa}$ (150 psi) pressure drop

85 Water seal direct stiffness forces for $10 \mu \mathrm{m}(.39$ mil $)$ shake amplitude.

86 Water seal direct stiffness forces for $10 \mu \mathrm{m}(.39$ mil) shake amplitude

87 Water seal cross-coupled stiffness forces for $10 \mu \mathrm{m}$ (.39 mil) shake amplitude

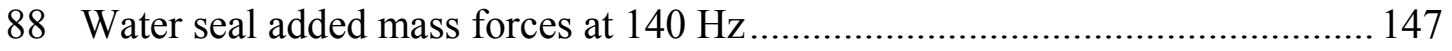

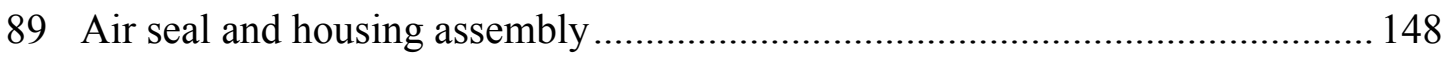

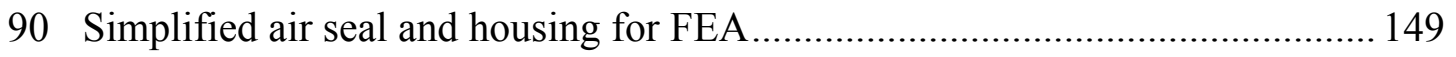

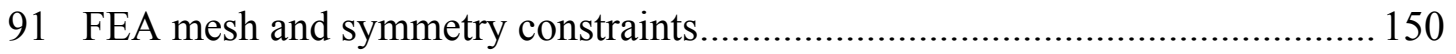

92 FEA bolt-hole constraints and pressure load ........................................... 151

93 FEA deflection results with displacement constraints ................................... 152

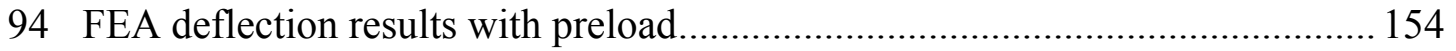

95 FEA Y-direction deflection results with preload …..................................... 155

96 FEA X-direction deflection results with preload .......................................... 155

97 FEA deflection results with preload and global friction ................................. 156

98 FEA Y-direction deflection results with preload and global friction................. 157 
FIGURE $\quad$ Page

99 FEA X-direction deflection results with preload and global friction................. 157

100 FEA von Mises stress results with preload and global friction......................... 158

101 XLTRC model for rotordynamic analysis................................................... 160

102 Rotordynamic root locus plots for no seal configuration............................... 161

103 Rotordynamic root locus plots for seals without preswirl configuration........... 162

104 Rotordynamic root locus plots for seals with preswirl configuration ................ 162

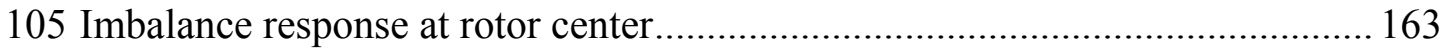

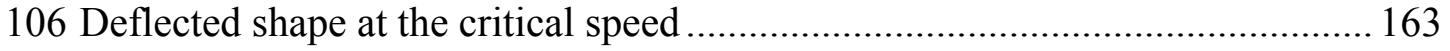

107 Rotordynamic root locus plot for seals without preswirl configuration ............ 165 


\section{LIST OF TABLES}

$\begin{array}{lll}\text { TABLE } & \text { Page }\end{array}$

1 Rotor natural frequency dependent on non-shaft component stiffness................26

2 Rotor natural frequencies for assembled rap tests................................................5

3 Typical FOSG calibration matrices and standard deviations ................................. 60

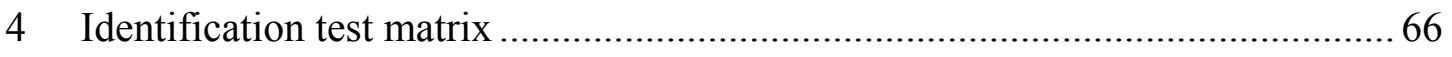

5 Frequency domain identification test results (metric units) …………………....... 84

6 Frequency domain identification test results (English units) ............................... 84

7 Eigenvalues of system model with frequency domain identification

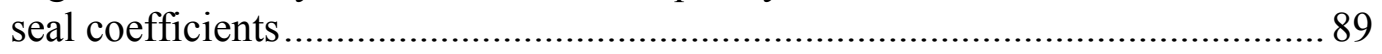

8 System parameters for OKID example ……………………………………....... 96

9 Natural frequencies and damping ratios from OKID example ............................. 97

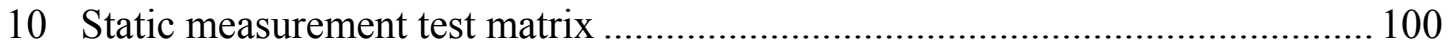

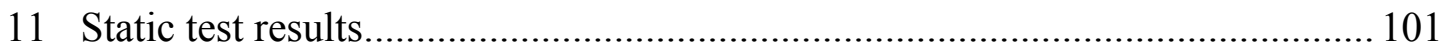

12 Comparison of test results with historical results ............................................ 104

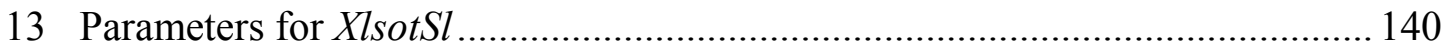

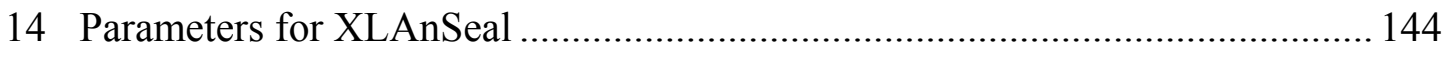

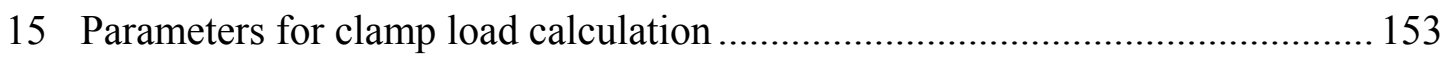

16 Operating conditions for rotordynamic analysis .............................................. 160

17 Damping ratios and damped natural frequencies for different seal configurations. 


\section{INTRODUCTION}

Each year, millions of dollars are lost due to repairs and downtime as a result of turbomachine failure. In a specific instance, ConocoPhillips' Ekofisk platform in the North Sea halted production for 150 days because of an unstable reinjection compressor. The estimated cost due to lost production was on the order of $\$ 150$ million. Turbomachine failure due to rotordynamic instability can be avoided by accurately modeling all system components, including rotors, bearings, seals, impellers, and support structure. However, valid models do not exist for all of these components. Current impeller models for centrifugal compressors have no physical basis and can not be extrapolated for design purposes. Despite predictions for stable operation, many compressors have been subject to rotordynamic instability. To improve the present models, impeller forces must be characterized. This requires parameter identification methods suitable for testing real compressors. This research project examines possible methods of identifying rotordynamic forces in real turbomachines.

Experimental research in rotordynamics, as in other fields, relies heavily on test measurements to characterize dynamic phenomena. Motions of both rotors and support structures are typically measured with proximity probes and accelerometers. These measurements are obtained directly; however, forces are more difficult to determine. Forces are typically measured with strain gauges and/or calibrated load cells, and they are sometimes calculated from inertial properties. Rotordynamic force measurement is complicated by the difficulty of measuring the force applied to machinery rotating at high speeds. Forces applied to the rotor by non-rotating parts such as bearings or seals can be determined by measuring support reaction forces and compensating for support motion.

This dissertation follows the style of ASME Journal of Turbomachinery. 
Another approach to force measurement in turbomachinery utilizes a modern technology know as magnetic bearings (MBs). Magnetic bearings have a unique potential for directly measuring forces applied to a rotor. A MB supports a rotor using a feedbackcontrolled electromagnet to levitate and stabilize the rotor about the centered position. The non-contact interface provides a method for applying forces directly from the stationary MB to the rotor. The applied force is a function of the air gap, the control current, and the magnetic properties of the materials. Historically, there have been attempts to measure the applied forces by (i) modeling the magnetic force using $\mathrm{MB}$ control currents and the rotor position, (ii) measuring the magnetic flux, and (iii) installing load cells within the bearings.

Recent efforts at the Texas A\&M University (TAMU) Turbomachinery Laboratory have focused on an alternative method of measuring forces in MBs. High-sensitivity fiberoptic strain gauges (FOSGs) are bonded to the surface of the magnetic core material within the MBs. FOSGs provide an accurate method of measuring strains and, as a result, the force applied by the MB. These measurements have been used to observe rotordynamic characteristics such as split natural frequencies associated with gyroscopic coupling.

Magnetic bearings clearly have a significant potential for characterizing the behavior of rotating machinery. MBs are used in applications including flywheel energy storage, high speeds machining, and turbomachinery. Some turbomachines, such as the centrifugal compressors mentioned previously, are available with MBs as the primary means of supporting the rotor. Installing FOSGs in these bearings would provide an opportunity for characterizing impeller forces and other rotordynamic phenomena.

The current research at the TAMU Turbomachinery Laboratory is focused on developing a parameter identification method suitable for identification in real turbomachinery. During the course of the present research, the installation of air seals on the existing test 
rig provides an opportunity for developing this parameter identification method. Air seals generate radial forces that can be modeled by stiffness and damping coefficients. Phenomena such as impeller forces in a compressor can be modeled in a similar fashion. The air seal parameter identification is, therefore, a useful representation of the process that would be required for identification in real turbomachinery.

This project examines frequency domain and time domain approaches to the identification problem. Static measurements of stiffness coefficient are performed using empirical formulas related to MB current and position measurements. The results are compared with predictions from a seal coefficient code and with test results from other test programs.

In addition, the present research seeks to compare MB strain measurements for quasistatic conditions with predictions from commercial finite element software. This effort seeks to better understand the physical mechanism producing the strain that allows for force measurement in MBs using FOSGs.

\section{Background Information}

Two modern technologies are utilized throughout this research: magnetic bearings and fiber-optic strain gauges. Combining these technologies allows for accurate force measurements with magnetic bearings. A general description of these technologies follows.

\section{Magnetic Bearings}

Fig. 1 displays the main components of a typical magnetic bearing (MB) [1]. MBs are increasingly used in rotating machinery because they offer several advantages over conventional bearings. A MB uses electrical currents to generate a magnetic field that levitates the rotor. As a result, there is no physical contact between the MBs and the rotor. This significantly reduces the power loss associated with the bearings and eliminates physical wear. Magnetic bearings can also support rotors at higher speeds 
than conventional bearings. Some MB machine tool spindles can rotate at speeds up to $100,000 \mathrm{rpm}$. MBs are also used in high-speed flywheel applications for energy storage. Controllability is another advantage. The bearing settings can be adjusted to produce desired characteristics and responses. In addition to varying standard parameters such as stiffness and damping, many bearing controllers have vibration control options that can produce rotating forces to counteract imbalance.

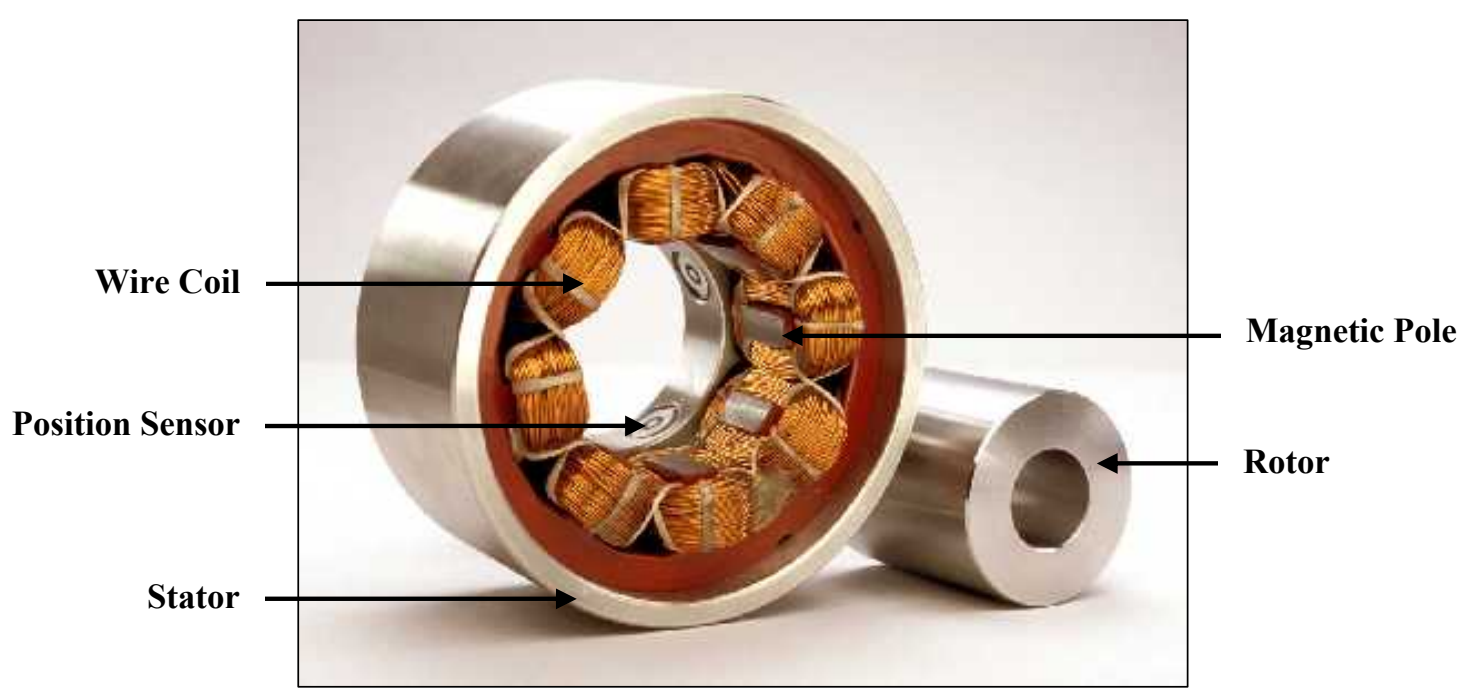

Fig. 1 Typical MB construction [1]

\section{Fiber-Optic Strain Gauge Technology}

Fiber-optic strain gauges (FOSGs), like conventional strain gauges, measure strain in materials. One end of the fiber optic is bonded to the surface of a material. The bonded ends of three fibers are shown in Fig. 2 [2]. The fiber has two reflective surfaces within the bonded region. Light is transmitted from the opposite end of the fiber to the surfaces and then reflected back, creating an interference pattern. As strain is produced in the material, the interference pattern changes, and a signal conditioning unit translates the pattern change into a voltage proportional to material strain. FOSGs are roughly 100 times more sensitive than conventional strain gauges. The optical signals are not 
corrupted by electrical and magnetic noise, which is very important for the present application. By bonding the FOSGs to the poles of MBs, reaction forces between the rotor and the bearing can be determined.

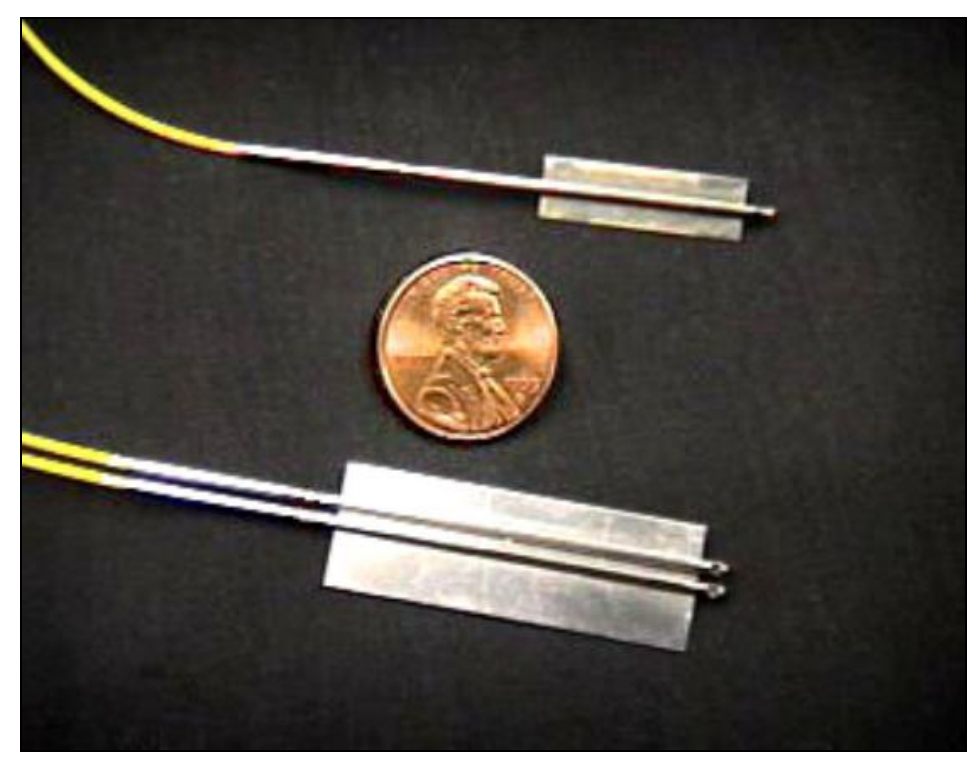

Fig. 2 Fiber-optic strain gauges [2]

\section{Methods and Results in Literature}

General information related to rotordynamics, seals, and rotodynamic testing is well documented in Turbomachinery Rotordynamics: Phenomena, Modeling, \& Analysis by Childs [3] and Rotordynamics of Turbomachinery by Vance [4]. Estimation topics are addressed in Optimal Estimation of Dynamic Systems by Crassidis and Junkins [5]. Frequency and time domain identification and their mathematical backgrounds are covered in Applied System Identification by Juang [6] and System Identification by Ljung [7].

The remainder of the literature review focuses on topics where the proposed research contributes significantly to the present body of knowledge. Historical attempts at force 
measurement in magnetic bearings have experienced limited success. Methods using magnetic flux sensors, load cells, and empirical current and position formulas have produced results with relatively large uncertainties. Fiber-optic strain gauge technology substantially improves the accuracy of the force measurements [8].

\section{Piezoelectric Load Cells}

Traxler and Schweitzer (1984) [9] mount piezoelectric load cells between the magnetic bearing housings and the test platform to measure reaction forces. Accelerations of the bearing housings produce forces that affect the reaction-force measurements. The housing forces are calculated using accelerometer measurements and are then subtracted from the measured total force to calculate the actual force applied to the rotor. The signal-to-noise ratio is low, and the inertial forces are large at higher frequencies.

Lee, Ha, and Kim (1994) [10] use a similar approach to perform system identification. They also experience large uncertainties. In general, this approach is problematic because the load cells must be sized to properly secure the bearings and must have an acceptable sensitivity for forces generated at high frequencies. This causes the low frequency results to have a poor signal-to-noise ratio. The high frequency results are suspect because the bearing motion increases with frequency, creating extraneous forces.

\section{Empirical Current and Position Formulas}

Matros, Sobotzik, and Nordmann (1996) [11] use an empirical formula relating the bearing currents and the rotor position to the applied force. Their formulas neglect eddy current loss, hysteresis, and magnetic saturation effects. Matros et al. model hysteresis and saturation properties in an effort to improve results. The force calculations are used to determine bearing and seal coefficients. In specific cases, stiffness is over-predicted by $8 \%$.

Fittro, Baun, Maslen, and Allaire (1997) [12] measure forces on a static test rig, varying eccentricity and force amplitude. They find that eccentricity changes contribute to most 
of the uncertainty in the results. The mean error distribution and standard deviation were $1 \%$ and $4 \%$ of the bearing load capacity, respectively.

\section{Magnetic Flux Sensors}

Gahler (1998) [13] uses hall sensors to measure the magnetic flux from the bearing poles. The rotor position and magnetic flux are related to the force with an empirical formula. A correction algorithm is implemented to correct for eddy currents, hysteresis, and saturation. Dynamic forces are applied at frequencies from 20 to $200 \mathrm{~Hz}$ with constant amplitude, and the force error is $\pm 11 \%$ of load capacity. Dynamic forces are then applied at $120 \mathrm{~Hz}$ for various amplitudes, and the force error is reduced to $\pm 2 \%$ of load capacity.

Knopf and Nordmann (2000) [14] use flux measurements to identify dynamic properties of hydrodynamic bearings. Uncertainties are around 1\% of load capacity for static measurements, but they deteriorate to $5 \%$ with increasing eccentricity and rotor speed.

Pottie (1999) [15] uses several methods to determine forces of magnetic bearings. A current and position dependent force model is attempted, and considerable time and effort are spent trying to map the model coefficients. Hall sensors are also attempted. A third method is accomplished by supporting the poles (not the bearing housings) with load cells. However load cells with sufficient sensitivity for accurate measurements allow the MB poles to move noticeably. Accelerometers are installed to compensate for the pole inertial forces. Unfortunately, this introduces new vibration modes and resonances. All of these methods are unable to significantly improve uncertainties over previous methods.

\section{Force Measurement in MBs at TAMU}

Raymer and Childs (2001) [16] use FOSGs to measure dynamic forces applied by an external exciter. This method results in dramatic improvements in uncertainty. The uncertainty is $4 \mathrm{~N}(1 \mathrm{lb})$ or $.1 \%$ of the bearing load capacity. In 2002, Pavesi [17] uses 
an empirical formula based on current and position to calibrate the FOSGs at low frequencies. The formula is believed to be sufficiently accurate at low frequencies because of the high repeatability of the results. This method encounters difficulties associated with parameter errors in the empirical formula, and the resulting uncertainties are not as low as in the method of Raymer and Childs.

Zutavern and Childs (2005) [18] develop a dynamic calibration for the FOSGs. In this method, the FOSGs are calibrated using rotor inertial force. The rotor inertial force is generated by exciting the rotor with frequencies below the first bending mode. The calibrated FOSGs are then used to characterize the split natural frequencies resulting from gyroscopic effects. The calibration has an uncertainty of $9 \mathrm{~N}(2 \mathrm{lb})$ or $.2 \%$ of the bearing load capacity, a reduction by a factor of 10 from Gahler's result [13].

FOSGs have produced results with the lowest uncertainties to date. Previous FOSG results provide justification for applying this technology to improve the knowledge of real turbomachinery. Using force measurements from FOSGs for parameter identification in the current test configuration will provide an excellent foundation for identification of rotordynamic forces in real turbomachinery. 


\section{TEST RIG DESCRIPTION AND DESIGN}

The test stand, magnetic bearings, and rotor are designed and assembled during previous research efforts. The sensors and data acquisition system are expanded to accommodate the current test program. Improvements include increasing the number of data acquisition channels, adding accelerometers, installing additional FOSGs, and installing an air temperature sensor. To study parameter identification, seals are added to the midspan of the rotor. This section describes the original test rig and the design process for the new hardware associated with adding seals for identification

\section{Test Rig Description}

The test rig consists of a rotor supported at either end by radial MBs. The rotor is driven by an electric motor, and the system is equipped with pneumatic brakes. The strain gauges are installed in both MBs. Data from the MB controller and the FOSG signal conditioning unit (SCU) are acquired using National Instruments hardware and Labview software. The MB Test Rig is displayed in Fig. 3.

\section{Test Rig}

The MBs (1) have a load capacity of $3560 \mathrm{~N}(800 \mathrm{lb})$ and support a steel rotor (2) weighing $2130 \mathrm{~N}(480 \mathrm{lb})$. Disks (3) provide substantial rotational inertia and, accordingly, gyroscopic coupling. The laminated sleeves (4) are the surface on which the magnetic force is exerted. Auxiliary bearings (5) support the rotor when it is not levitated. The coupling (6) and the quill shaft (8) are both shielded for safety. The brakes (7) can be used to rapidly decelerate the system if the rotor delevitates. A pulley (9) and drive belt transmit power from the motor. The test stand base (10) is constructed of $19 \mathrm{~mm}$ (.75 in.) steel plates with a $76 \mathrm{~mm}$ (3 in.) steel top. 


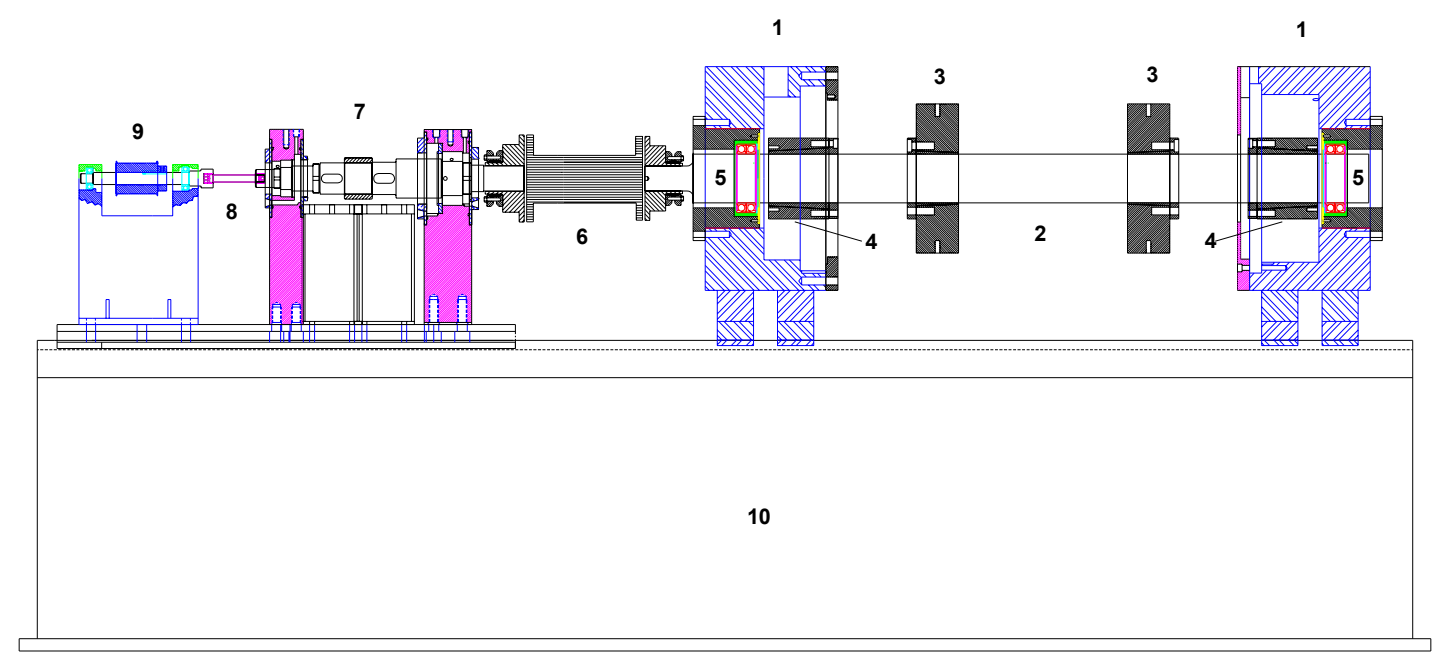

Fig. 3 Magnetic bearing test rig at TAMU Turbomachinery Laboratory

\section{NI Hardware and Labview Data Acquisition Software}

The data acquisition system consists of 2 E-series National Instruments PCI cards. The 6035E card has 2, 12 bit, analog output channels and 8, 16 bit, differential type analog input channels. The $6036 \mathrm{E}$ has 2, 16 bit, analog outputs channels and 8, 16 bit, differential type analog input channels. Each E-series card is connected to an external SC2040 card, which allows the analog inputs for each board to be acquired simultaneously. By connecting the two E-series cards with a RSTI cable, clock signals can be routed from one board to another. This allows for the synchronization of the analog inputs and analog outputs of both cards.

Data points are sampled at $10 \mathrm{kHz}$ for all dynamic measurements. The analog output waveforms, used to excite the test rotor, are updated at $5 \mathrm{kHz}$. More information on the data acquisition system and its operation is available in Zutavern [8].

Labview data acquisition software is used for all dynamic testing. A variety of Virtual Instruments have been developed for tasks including FOSG calibration, waveform generation and refinement, and seal testing. With the input and output capabilities of the 
data acquisition hardware, the Labview programs can excite the test rotor by transmitting analog waveforms to the MB controller and measure positions, accelerations, and strain concurrently.

\section{Magnetic Bearings and MB Controller}

The test rig magnetic bearings are donated to the research program and originally come from a natural gas compressor. An end view of one the magnetic bearings is displayed in Fig. 4. From the figure, 16 wire coils are visible. The coils are wound so that the 4 coils in each quadrant of the MB act in unison. The result is 4 groups of poles per MB. The centerline of each group is oriented at $45^{\circ}$ with respect to the horizontal plane. Since the rotor material is not permanently magnetized, the poles of the MB can only attract (not repel) the rotor, the pole groups act in opposing pairs. The currents in the $\mathrm{MB}$ are controlled by a feedback controller acting to stabilize the rotor.

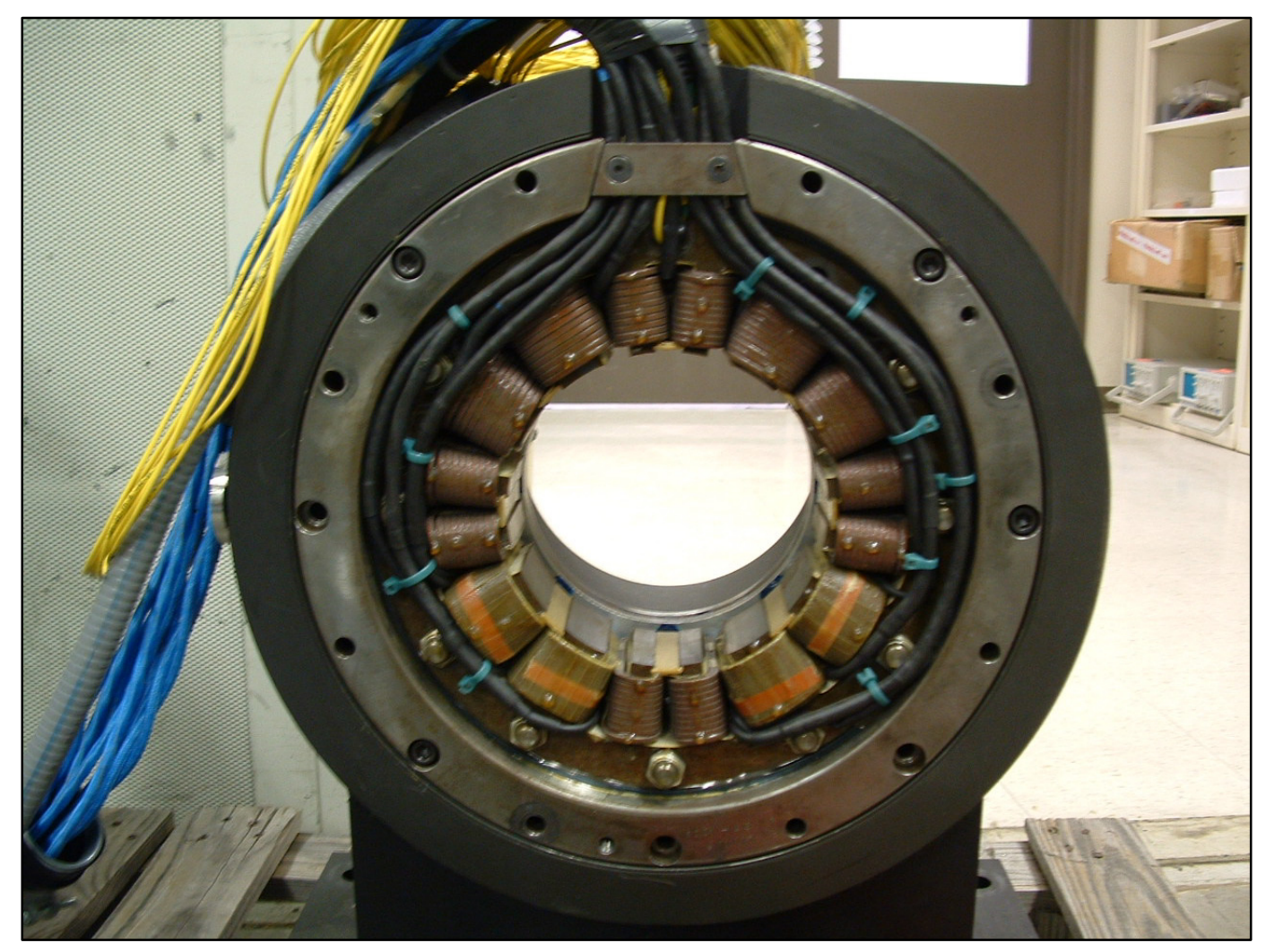

Fig. 4 Magnetic bearing end view 
The magnetic bearing controller is the MBControl module produced by SKF Magnetic Bearings. This module uses position measurements from proximity probes to determine the current response required to levitate the rotor. The MBControl module controls amplifiers that produce the required currents. In addition, the module interfaces with a PC through a serial link and MBScope software that is installed on the PC. The module also creates analog outputs through the MBResearch panel. These outputs are analog voltage signals that indicate rotor position and control currents.

The MBResearch panel also has analog inputs that the MBControl module can use to adjust either target rotor position or the control currents. The analog input must be activated using the MBScope software. Analog waveforms generated by Labview are transmitted to the MBResearch panel inputs. This allows the researcher to excite the test rotor using MBs. Typical rotordynamic testing uses imbalance or impact loads to excite the rotor, or shakers to excite the stator. MBs have a unique ability to function as calibrated exciters while simultaneous supporting the rotor. This results in a high degree of flexibility for a MB test rig.

\section{FOSG Locations}

The FOSGs are installed on the MB poles. Fig. 5 displays a close-up view of one of the MB poles, with two FOSGs visible. One FOSG is bonded to the magnetic material, or laminate, of the MB. The other is not bonded but is used in previous research to monitor temperature changes in the $\mathrm{MB}$. 


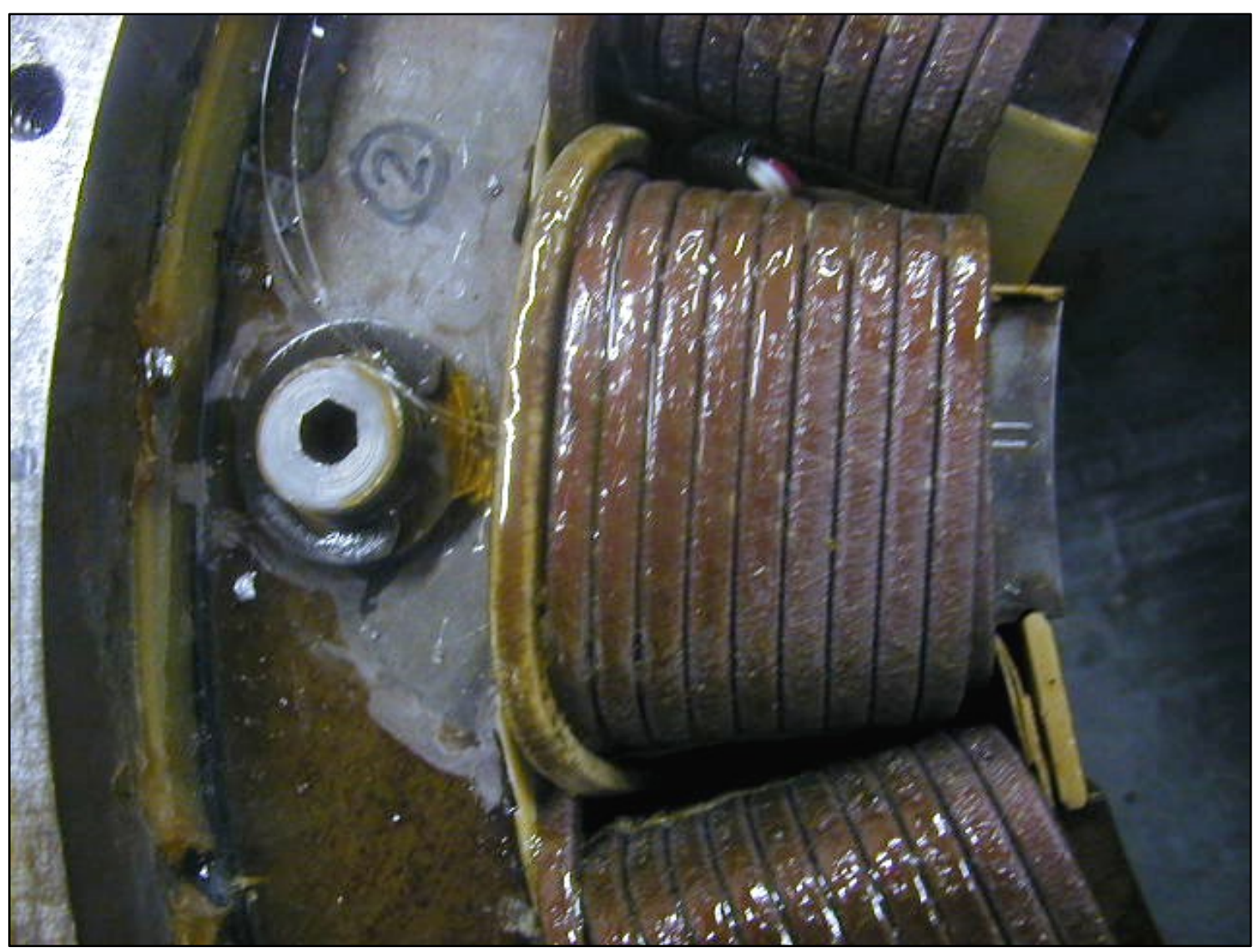

Fig. 5 FOSG installation in a MB

The previous sections describe the aspects of the test rig which have existed prior to the present research. The first step in preparing for the parameter identification research is to install additional FOSGs. The test rig has four original FOSGs bonded to the poles of the non-drive MB. For the present research, the drive end $M B$ is equipped with 4 FOSGs. For both bearings, the FOSGs are oriented as shown in Fig. 6. The FOSGs are offset from the centerline of the pole groupings; however, poles within a group act in unison; accordingly, the strain measurement is representative of the net force applied by the pole group. 


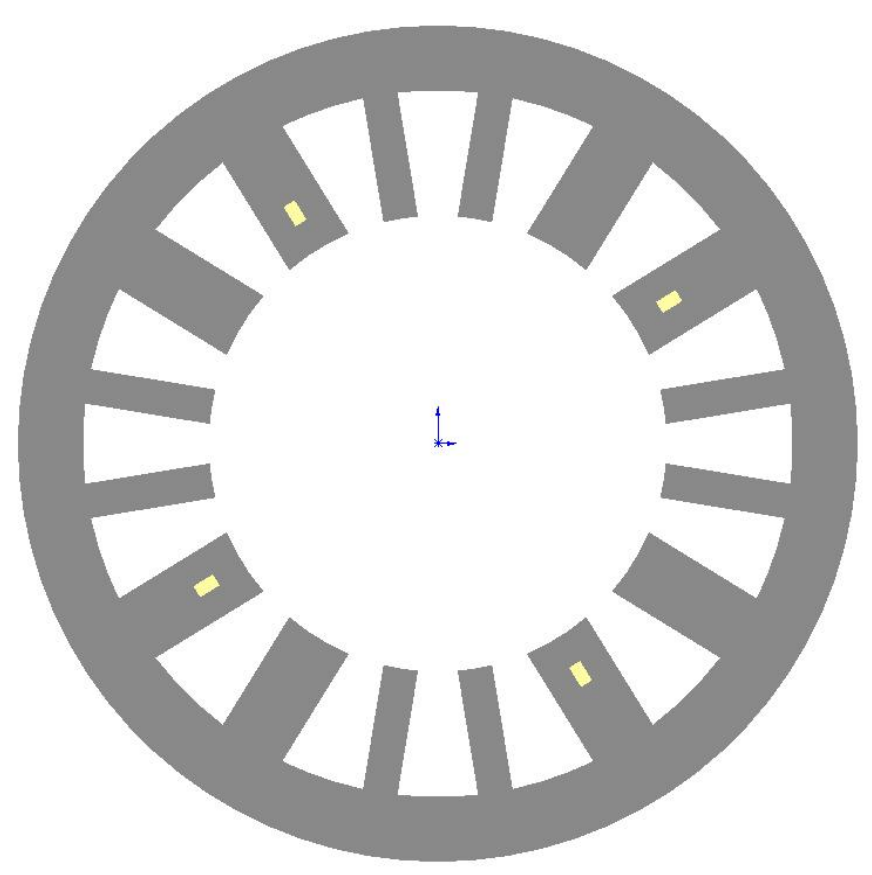

Fig. 6 FOSG locations

The installation of the new FOSGs allows force to be measured at both end of the rotor. The combination of force and position measurements provides the necessary information for parameter identification. The remainder of the section focuses on the hardware design and installation associated with the addition of a seal to the test rig.

\section{Air Seal Final Design}

To reiterate, the overall purpose of the magnetic bearing test rig is parameter identification in real turbomachines. A major step toward this end is performing parameter identification on the current test rig. Modifying the test rig by adding back-toback annular gas seals at the axial center of the rotor could produce suitable forces for identification. The first step in the design process is to consider what type of seal, water or air, is most suitable for achieving this objective. An air seal with a radial clearance of $305 \mu \mathrm{m}(.012 \mathrm{in})$ and a length of .102 $\mathrm{m}$ (4.0 in) is selected based on the analysis 
detailed in Appendix A. The present section discusses the final design of the air seal installed on the MB test rig.

The smooth air seals are now added to the test rig to introduce rotordynamic forces for identification. The seal assembly shown in Fig. 7 and Fig. 8 is located at the rotor midspan. The smooth air seal halves $(1,2)$ are clamped by the lower housing $(3)$ and the upper housing (4). The end seal halves $(5,6)$ have exhaust ports that divert the air away from the test rig. The base plate (7) supports the stands (8) that bolt to the lower housing. The adapters $(9,10)$ are used to center the seals about the rotor. Swirl rings (11) are pressed into the air seals to control the preswirl conditions. The importance of the swirl rings is discussed later.

Fig. 9 shows the test rig with the air seal assembly installed. Pressurized air at $1.7 \mathrm{MPa}$ ( $250 \mathrm{psi}$ ) is supplied to the annulus behind the swirl races. The air seals have a $300 \mu \mathrm{m}$ (.01 in) radial clearance with the rotor that restricts the air flow. Perturbing the rotor or stator results in radial seal forces. The physical mechanisms generating the seal forces are described in [3]. The seal forces are modeled for small perturbations with two degree-of-freedom stiffness and damping matrices. The degrees of freedom correspond to two perpendicular radial directions. Seal force models are discussed in more detail later. 


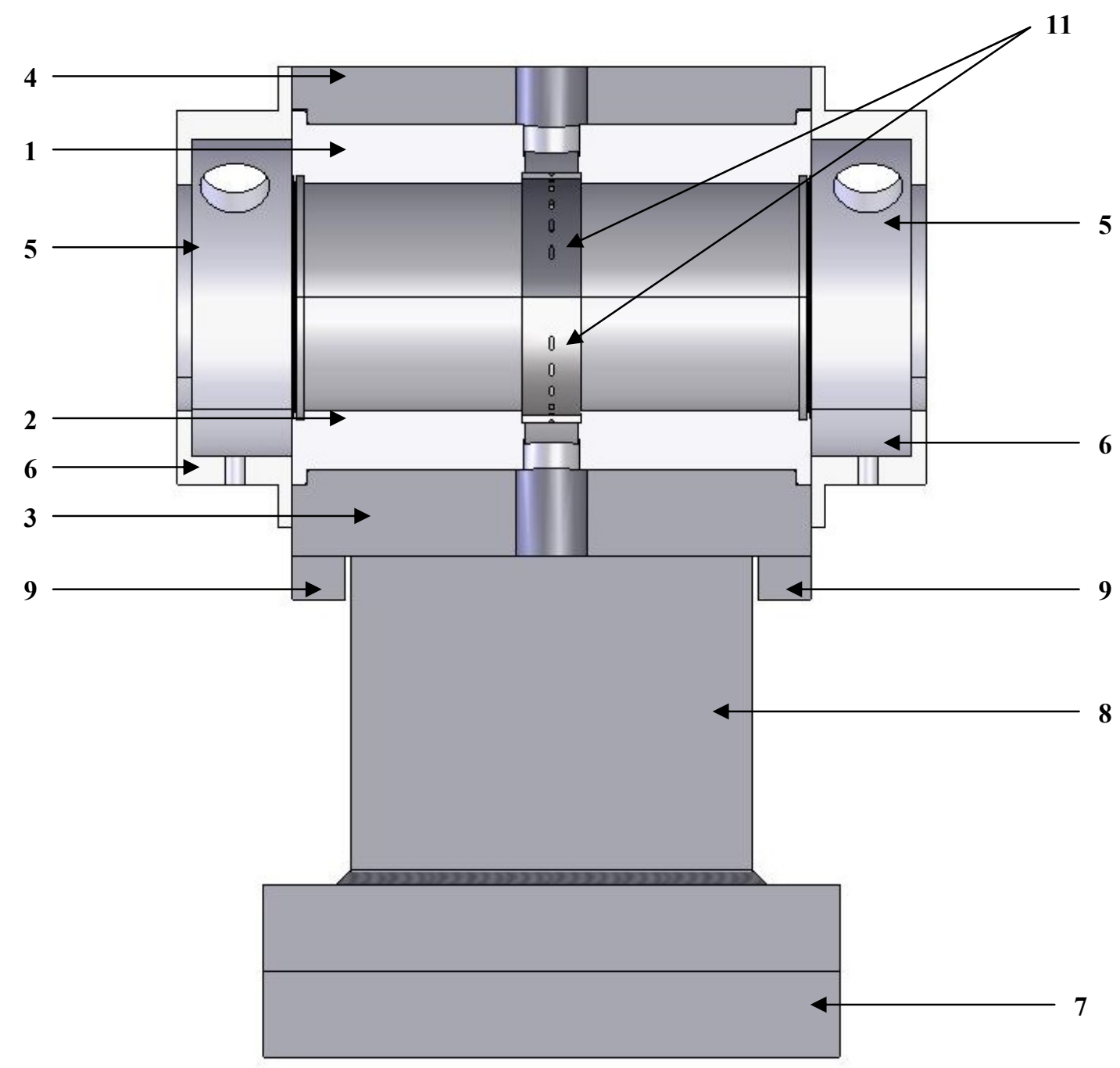

Fig. 7 Section view of air seal assembly 


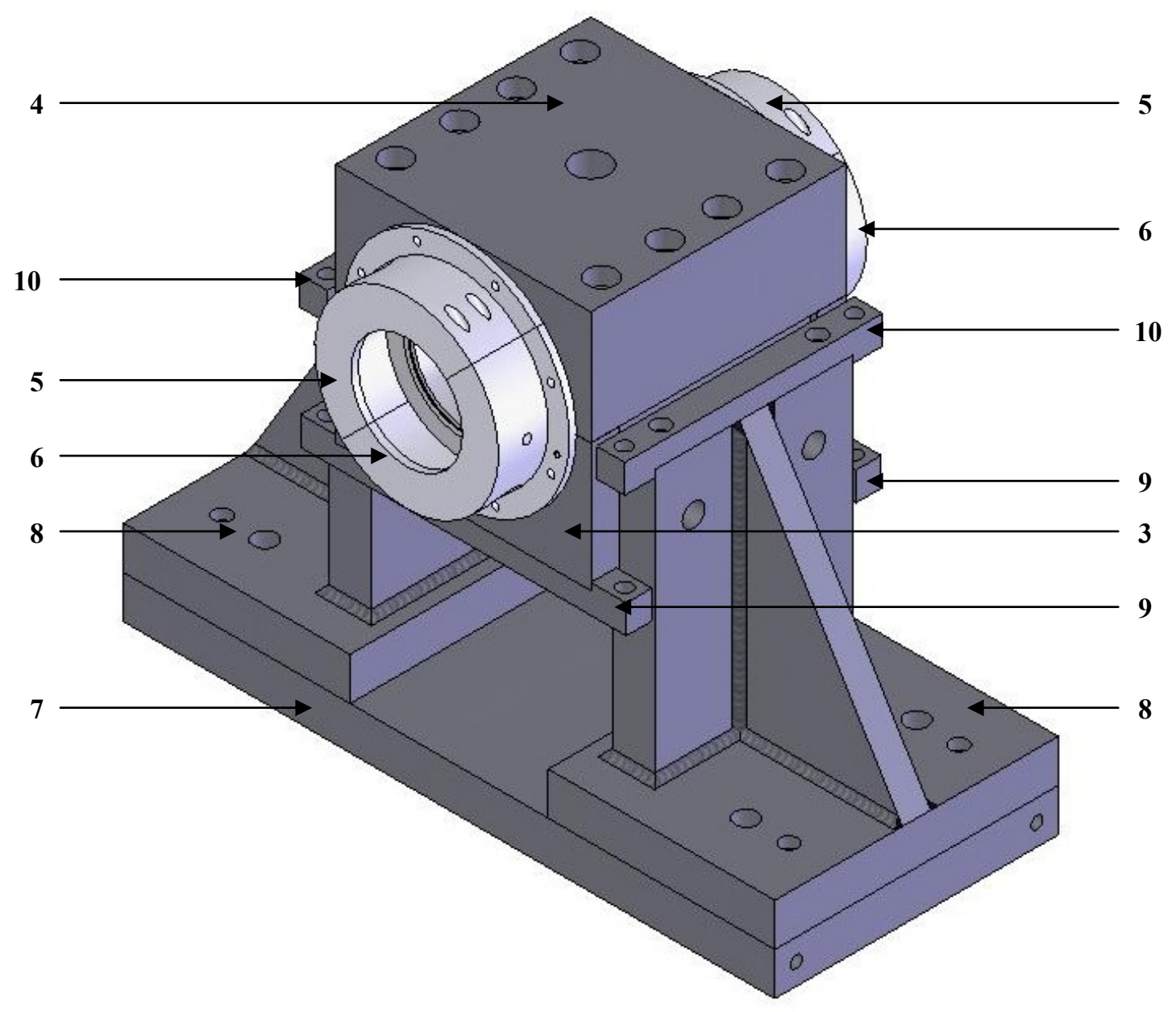

Fig. 8 Isometric view of air seal assembly 


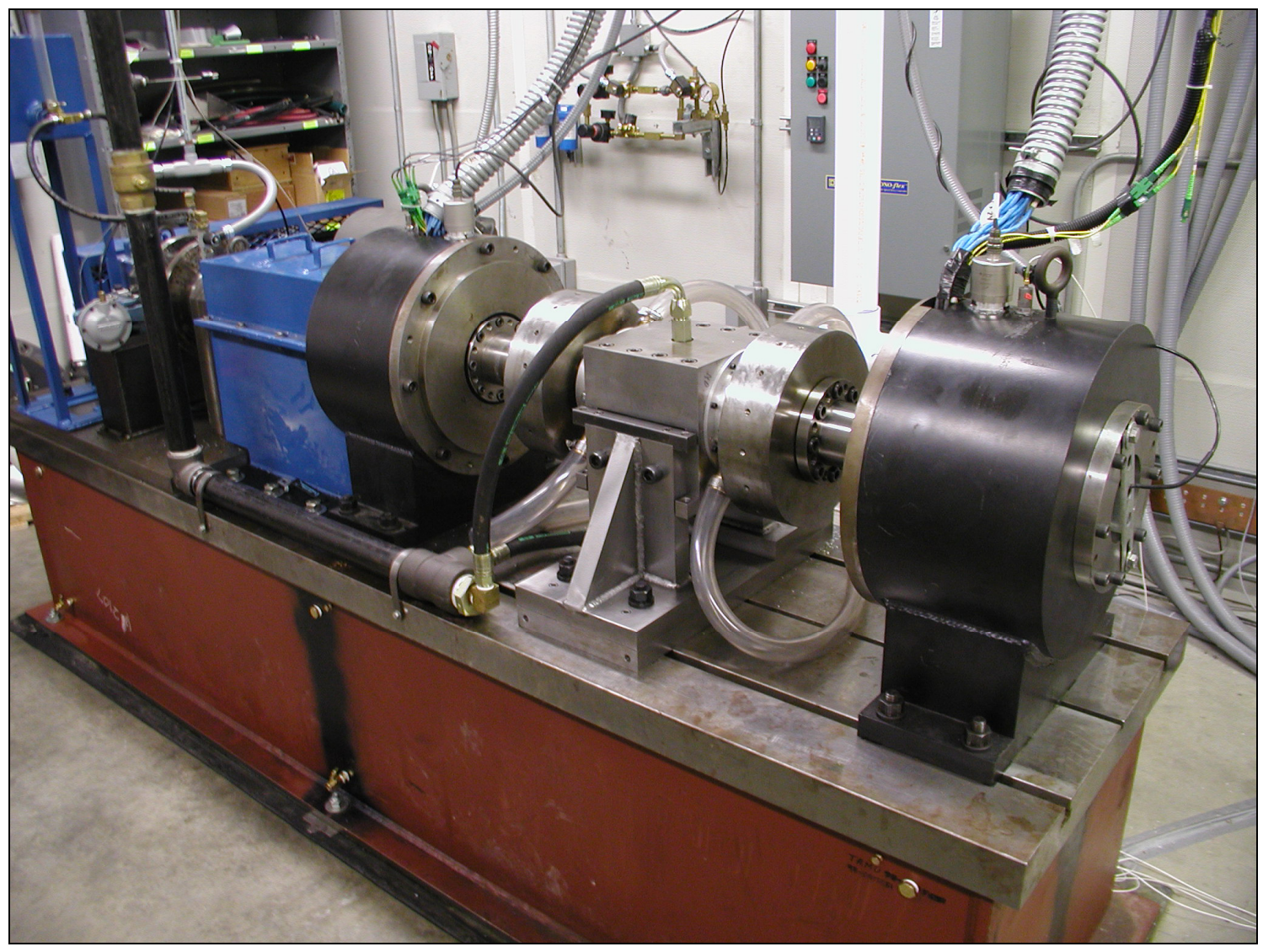

Fig. 9 MB test rig with air seal assembly installed 


\section{THEORY OF OPERATION}

This section focuses in more detail on the theory behind several aspects of the test rig. FOSG operation is explained. MB operation and the appropriate test model are further developed. A calibration method for the FOSGs is derived.

\section{FOSG Theory}

The FOSGs connect to the FOSG signal conditioning unit (SCU). This device creates the laser light that is directed into the fiber optic cables. The light travels down the cables and reaches the end where the strain gauge is bonded to the MB. Fig. 10 demonstrates that the laser light is partially reflected by two surfaces typically spaced 12 $\mathrm{mm}(0.47 \mathrm{in})$ apart. The reflected light travels back through the fiber to the SCU. A Fabry-Perot interferometer technique is utilized to determine the phase shift resulting from the travel length difference of the light. The phase shift is related to the distance between the two surfaces. As the FOSG is strained, the distance between the surfaces changes, the phase of the light changes, and the strain is detected. The SCU output voltage signals indicate the strain. The voltages are recorded by the data acquisition system [19].

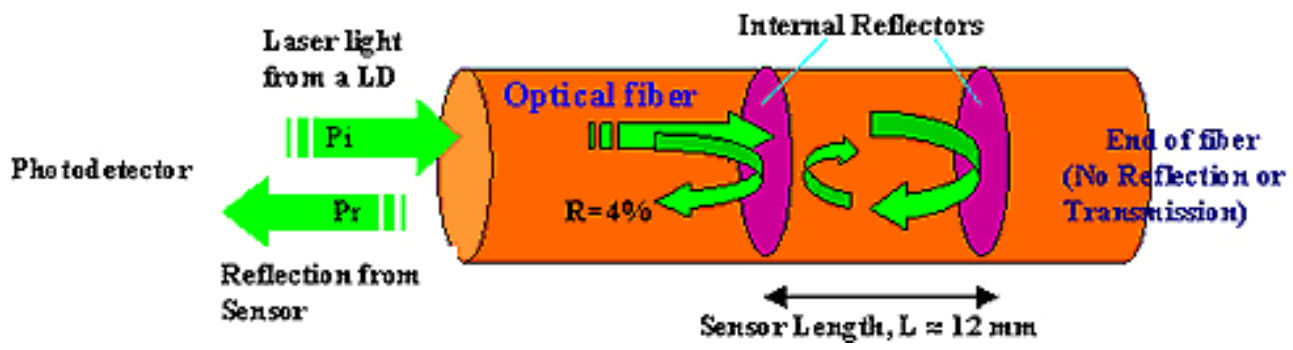

Fig. 10 Fiber-optic strain gauge diagram

The FOSG locations are displayed in Fig. 11 as seen from the non-drive end. Each FOSG is bonded to one of the primary MB poles. These poles do not lie on the axes used by the MBs. However, each pair of primary poles and the adjacent auxiliary poles 
act in unison. This causes the net force exerted by a given pole to occur along an axis. The result is that the FOSG detects the net force exerted by a pole along the poles respective axis.

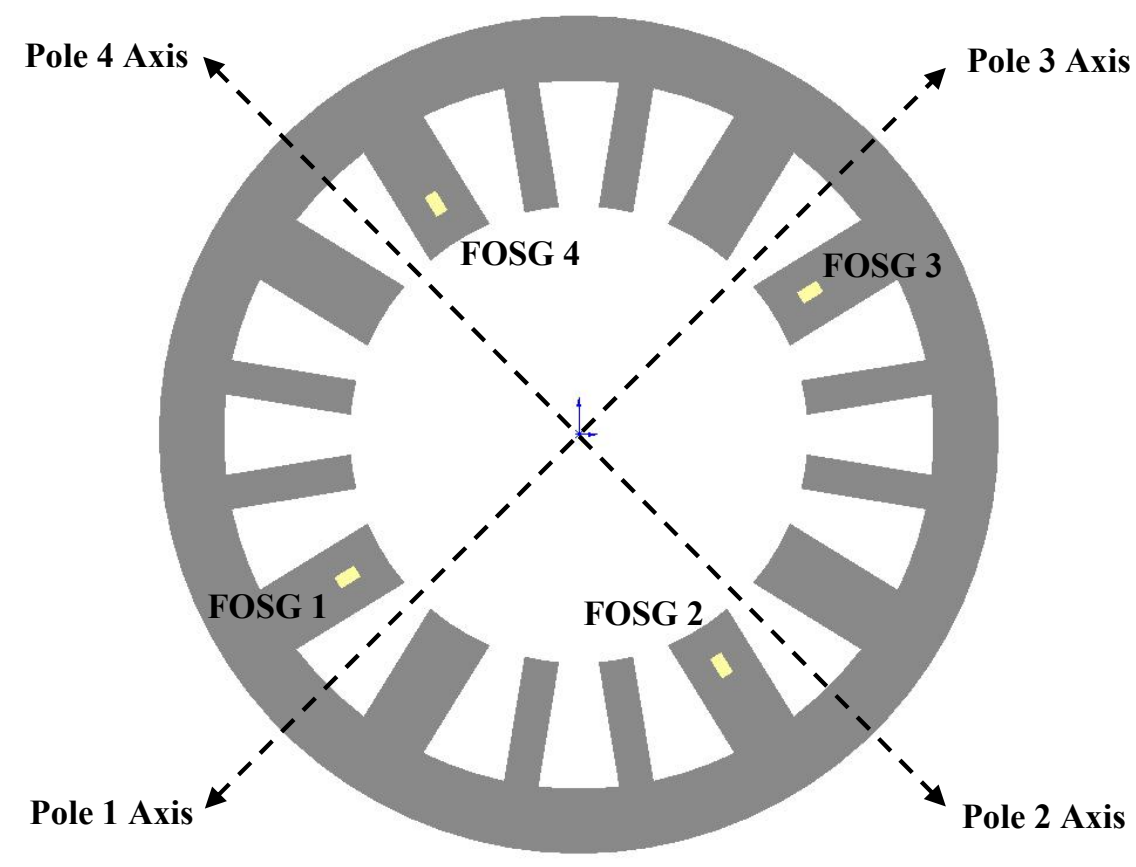

Fig. 11 FOSG locations

The strain measurement reflects the force applied to the rotor by each group of poles. The winding of the poles ensures that the individual forces applied by each pole scale proportionally within each group. An additional assumption arises from the fact that the SCU has only four channels. For tests requiring force measurements at both bearings, this means that only two FOSGs are used per MB. The operation of the MB controller ensures that responses of opposing pole groups also scale proportionally and in a repeatable fashion. 


\section{Magnetic Bearing Theory}

Magnetic bearings utilize a basic principle of electricity and magnetism whereby a magnetic field is generated from the motion of charges. The cross product of the coil current and the position vector from the current to a designate point describes the magnetic field generate at that point. The core material of the MB is a ferromagnetic material that enhances the magnetic field. A detailed explanation of the interaction between the magnetic field and the materials of the MB can be found in a later section.

The interaction between an electromagnet and an object composed of magnetic material contains an unstable equilibrium. Consider an attempt to suspend or levitate the object below the electromagnet. There is a point at which the attractive force applied to the object by and electromagnet is equal and opposition to the force of gravity acting on the object. This point is an unstable equilibrium because a slight increase in the gap between the magnet and the object causes a decrease in the attractive force allowing the object to fall. A decrease in the gap causes an increase in the attractive force, pulling the object into direct contact with the magnet.

This equilibrium can be stabilized by using a feedback controller to adjust the current of the electromagnet. The inertia of the object creates a lag between the change in force and the change in position of the object. This affords the controller time to adjust the current and drive the object back to the equilibrium position. Fig. 12 shows a block diagram for a single axis MB. A prefilter input specifies the target position of the rotor. The error between the target and actual position is the input for the controller. A PD controller is depicted in the figure; however, the MB controller also includes integral control, various filters, and other options such as adaptive control. The controller outputs a voltage, which can be adjusted by a post filter input to excite the system.

The voltage is transmitted to the current amplifiers where the currents for the MB poles are generated. The relationship between the current and the force applied to the rotor is 
highly nonlinear for low current levels and current levels nearing magnetic field saturation. A DC current is used create an operating point from which small perturbations in current result in linear changes in force. The linearized relationship is modeled with a magnetic bearing gain $k_{i}$. The force is applied to the rotor system, and the response of the system is measured with proximity probes.

The final significant feature of the MB model is the change in applied MB force as a function of rotor position. This too is a nonlinear relationship that is linearized for small motions about the operating point. A negative stiffness term $k_{x}$ represents the change in force applied to the rotor resulting from a change in position.

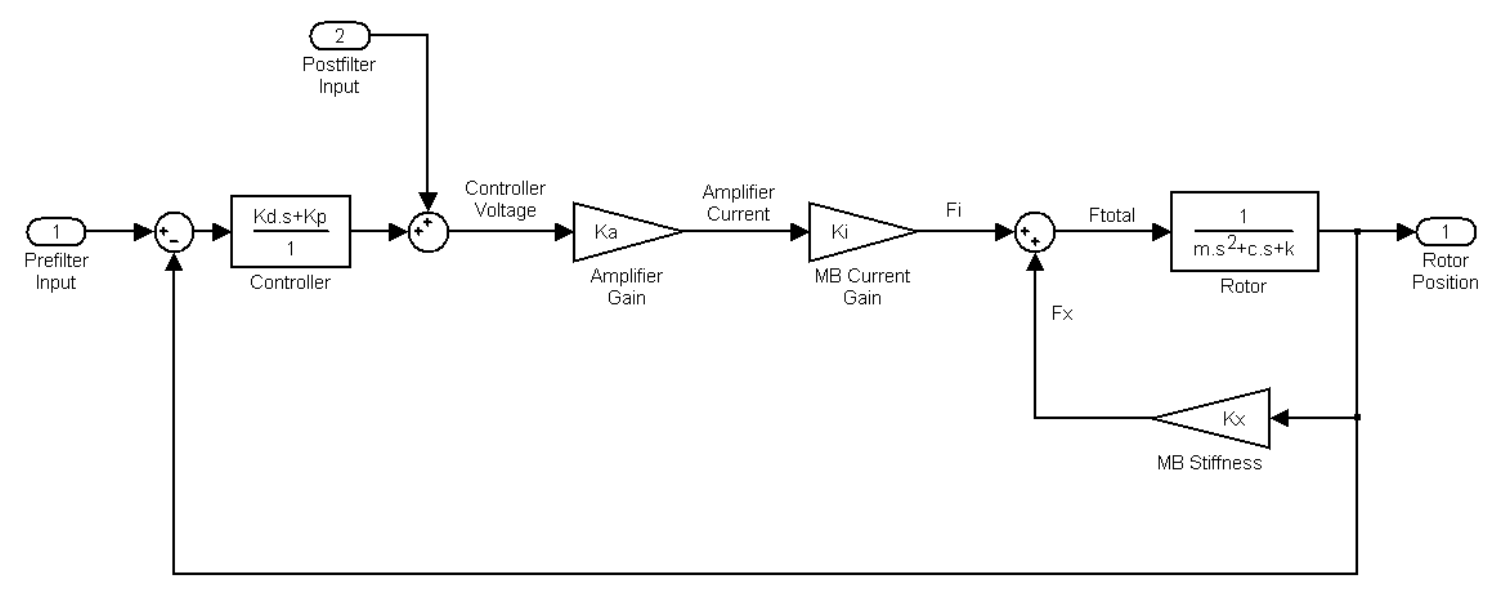

Fig. 12 Single axis MB model

The transfer functions of the system highlight some of the advantages of MBs. A basic rotor system including damping and stiffness terms from a conventional seal or bearing is described by Eq.(1).

$$
T_{1}=\frac{1}{m \cdot s^{2}+c \cdot s+k}
$$


The transfer function from amplifier current to rotor position is display in Eq. (2). It can be seen here that the MB stiffness associated with the change in rotor position $k_{x}$ is negative in sign and acts to destabilize the system.

$$
T_{2}=\frac{T_{1} \cdot k_{i}}{1-T_{1} \cdot k_{x}}=\frac{k_{i}}{m \cdot s^{2}+c \cdot s+k-k_{x}}
$$

In Eq. (3), the controller $C(s)$ and the amplifier $k_{a}$ gains are included. The combined controller and amplifier gains, $\bar{k}_{d}$ and $\bar{k}_{p}$, can be adjusted to arbitrarily place the eigenvalues of the system.

$$
T_{3}=\frac{C \cdot k_{a} \cdot T_{2}}{1+C \cdot k_{a} \cdot T_{2}}=\frac{\bar{k}_{d} \cdot s+\bar{k}_{p}}{m \cdot s^{2}+\left(c+\bar{k}_{d}\right) \cdot s+k-k_{x}+\bar{k}_{p}}
$$

The closed-loop characteristic equation is displayed below. The controller gains can stabilize the system despite the negative stiffness $k_{x}$. The controller can even stabilize the system if the rotor system has negative stiffness and/or damping terms.

$$
m \cdot s^{2}+\left(c+\bar{k}_{d}\right) \cdot s+k-k_{x}+\bar{k}_{p}=0
$$

The example is simplified from the true system used for conducting research. However, it captures the major aspects associate with MB control design. Note that this system is a representation of how the full system operates. The model changes significantly as it is considered for identification. The appropriate identification system model is addressed in the following sections.

\section{Validation of MB Model for Identification}

The appropriate model for an MB acting as a calibrated exciter has been the subject of some debate. The reference by Gahler [13] suggests, "Since the bearing forces can be measured, all forces acting on the rotor are known, and the free-free behaviour of the 
rotor can be measured." Before attempting to explain the appropriate model physically, a scientific approach to answering this question is explored. A series of predicted responses are compared with experimental responses to determine the appropriate model for each scenario.

The previous section demonstrates that controller gains for the MBs can be adjusted to place the eigenvalues of the system. The question arises as to whether the forces exerted by the controller response should be considered separately from the excitation forces that are typically applied by injecting a post-filter signal (Fig. 12). In other words, do the strain measurements detect the total force applied by the bearing to the rotor or only the force resulting from the excitation waveform.

The Turbomachinery Laboratory FE rotordynamics software, XLTRC ${ }^{2}$, is used to compute the force response of the rotor system (without the air seals) for comparison with experimental testing. Initially, the rotor model is analyzed and the natural frequency of interest is compared with results of previous experimentation. Next, different rotor-bearing models are considered, and transfer functions from force to position are determined for each model.

\section{Rotor Model and Natural Frequency Prediction}

The rotor model, shown in Fig. 13, is used in the seal selection and design analysis. It is now further refined to better represent the characteristics of the rotor. The rotor model is developed from the physical geometry of the coupling (1), laminations stacks (2), discs (3), and shaft (4). The densities of the parts are adjusted slightly to agree with component weights determined during the most recent rotor balancing. The modeled mass of the rotor, excluding the coupling, is $212 \mathrm{~kg}$ (468 lb) which is accurate to within $.5 \mathrm{~kg}(1 \mathrm{lb})$. The coupling mass used in the model is an effective mass calculated from the actual mass and the kinematics of the coupling motion. The effective coupling mass is $9.71 \mathrm{~kg}(21.4 \mathrm{lb})$, accurate to within $0.05 \mathrm{~kg}(0.1 \mathrm{lb})$. 


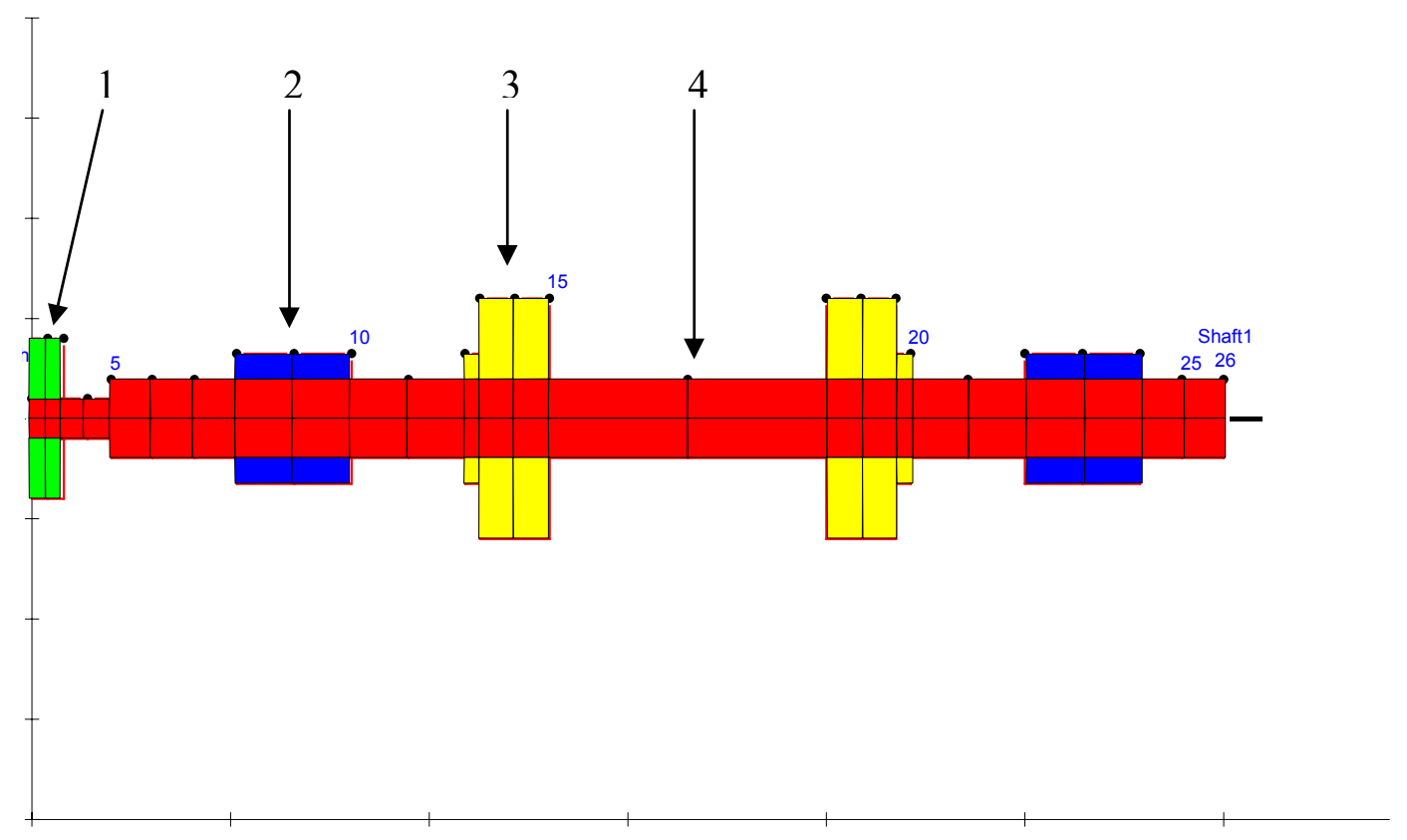

Fig. 13 XLTRC rotor model

Preliminary experimentation revealed that the natural frequency of the physical rotor is $140 \mathrm{~Hz}$. Initially, only the shaft model includes stiffness of $200 \mathrm{GPa}\left(29 \mathrm{x} 10^{6} \mathrm{psi}\right)$; however, this results in a prediction for the natural frequency of $132 \mathrm{~Hz}$. Including full stiffness for the other components results in a natural frequency of $157 \mathrm{~Hz}$. It is determined that using a $1 \%$ of material stiffness, 2 GPa $\left(29 \times 10^{4} \mathrm{psi}\right)$, for non-shaft components results in a natural frequency of $140 \mathrm{~Hz}$. The results are shown in Table 1. The effect of the stiffness on the natural frequency is clearly non-linear. The interpretation is that a slight increase in non-shaft component stiffness substantial stiffens the rotor assembly in the regions of the respective components. The result is that the rotor becomes relatively rigid at these locations, and further increases in the component stiffness do not continue to cause large increases in the rotor natural frequency. 
Table 1 Rotor natural frequency dependent on non-shaft component stiffness

\begin{tabular}{|c|c|c|c|}
\hline $\begin{array}{c}\text { Non-shaft Component } \\
\text { Stiffness (\%) }\end{array}$ & $\begin{array}{c}\text { Elastic Modulus } \\
\mathbf{( G P a )}\end{array}$ & $\begin{array}{c}\text { Shear Modulus } \\
\mathbf{( G P a )}\end{array}$ & $\begin{array}{c}\text { Rotor Natural } \\
\text { Frequency (Hz) }\end{array}$ \\
\hline 0 & 0 & 0 & 132 \\
\hline 1 & 2 & $0.77 \times 10^{4}$ & 140 \\
\hline 50 & 100 & $38 \times 10^{6}$ & 156 \\
\hline 100 & 200 & $77 \times 10^{6}$ & 157 \\
\hline
\end{tabular}

The damped natural frequency map is displayed in Fig. 14. The predicted separation correlates with previous $X L T R C^{2}$ models and experimentation [8,17]. The accuracy of the rotor model is acceptable for the present analysis. The forced responses can now be computed for comparison with experimental results.

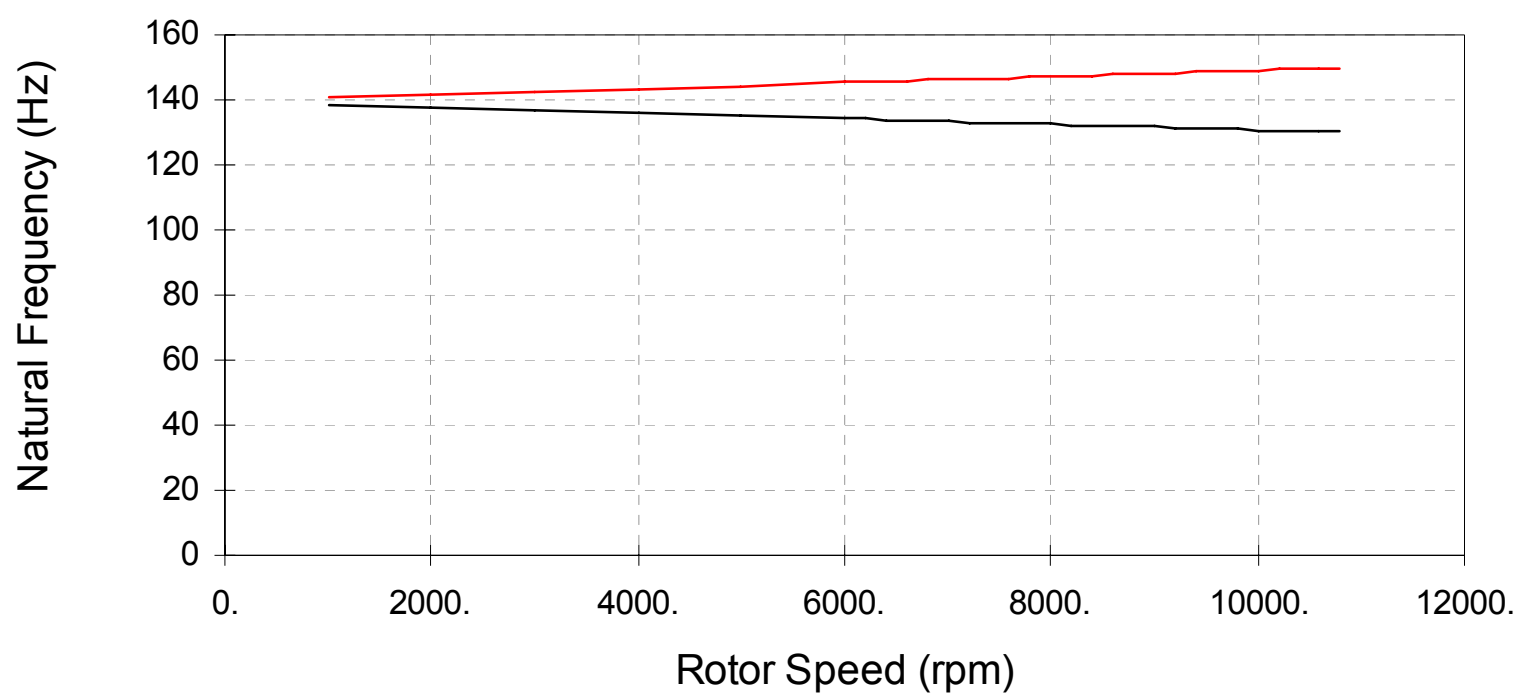

Fig. 14 Damped natural frequency map 


\section{Two Bearing System Model}

Four system models are now considered, and their responses are predicted. The first system is the two bearing system model is displayed in Fig. 15. For this analysis, both MBs are included in the model. The MBs are modeled with a negative direct stiffness, representing the change in force due to a change in rotor position, and transfer functions, representing the controller dynamics. The stiffness values and transfer functions are specified by SKF Magnetic Bearings. An imbalance is applied at either bearing and the response is used to compute the forced response. The Gyroscopics option in XLTRC ${ }^{2}$ is disabled because these results are compared with non-rotating tests.

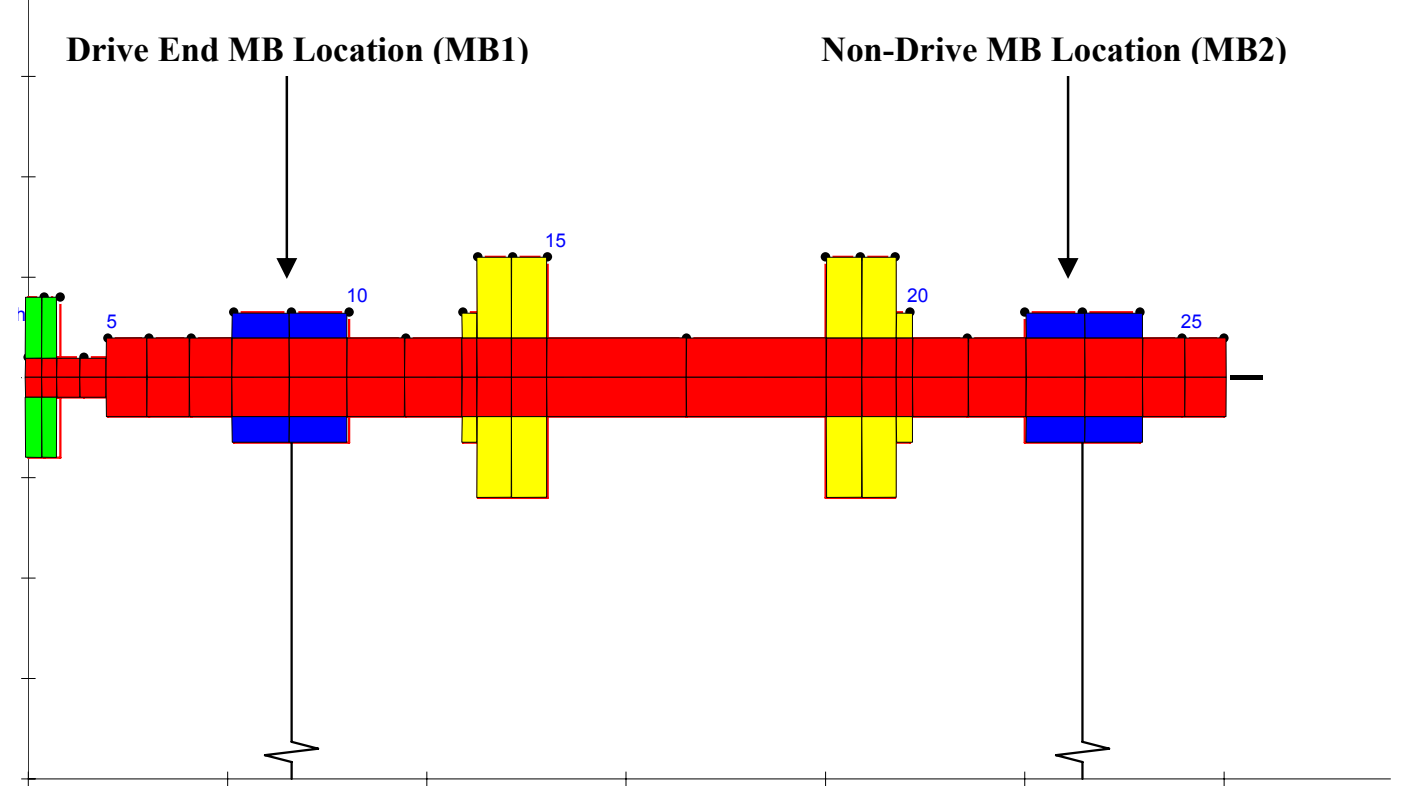

Fig. 15 Rotor-bearing system model with both bearings

An imbalance is applied first at the drive-end bearing (MB1) and then at the non-drive bearing (MB2). The responses at both bearings are computed for each case. The transfer functions from force to position are computed by dividing the response $R$ by the imbalance force $F$ as shown in Eq. (5). The imbalance force is equal to the product of the imbalance mass $m$, the imbalance radial location $u$, and the square of the rotor speed 
$\Omega$. Fig. 16 through Fig. 19 display the XLTRC predictions for the two bearing system model. The predictions do not include vertical and horizontal coupling because this analysis is non-rotating and the bearings are isotropic.

$$
G=\frac{R}{F}=\frac{R}{u m \Omega^{2}}
$$

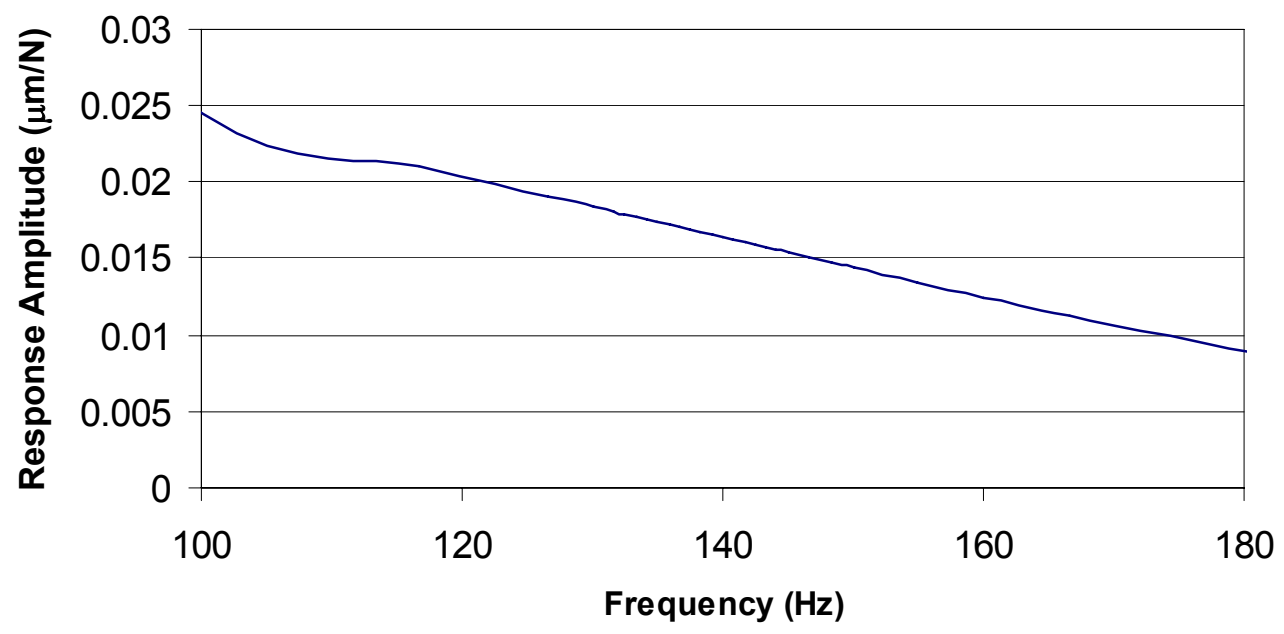

Fig. 16 Two-bearing response at MB1 to force at MB1 force 


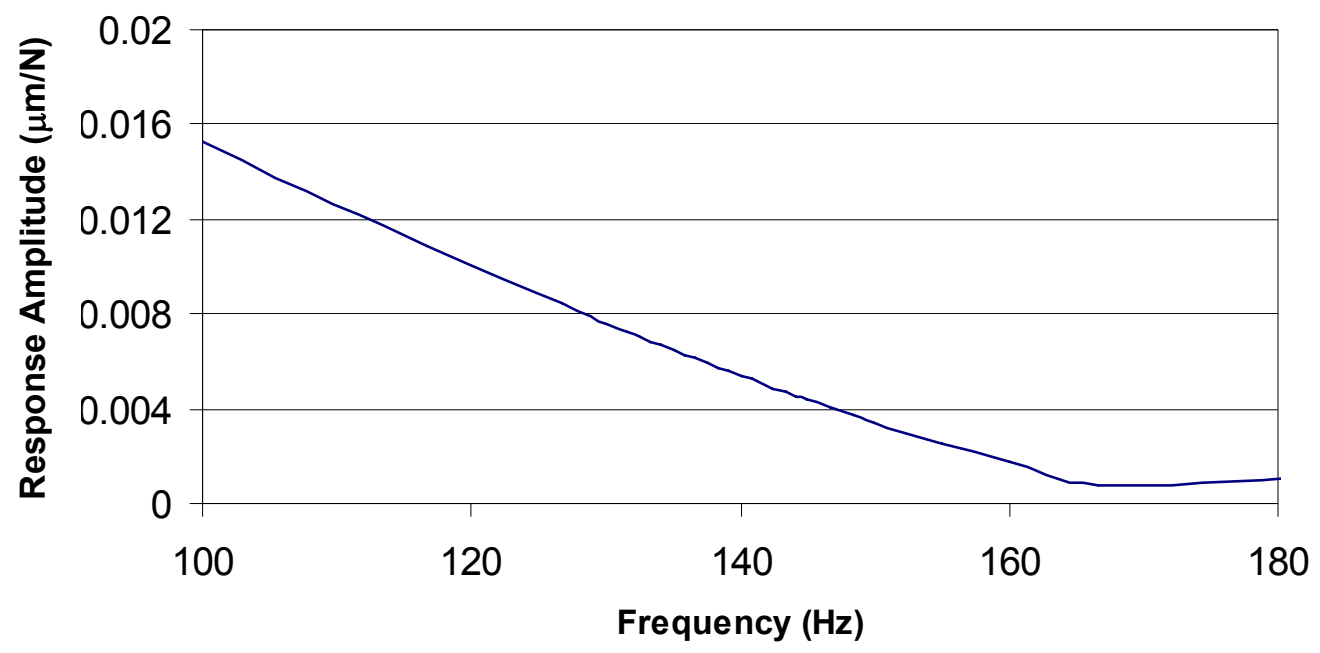

Fig. 17 Two-bearing response at MB2 to force at MB1 force

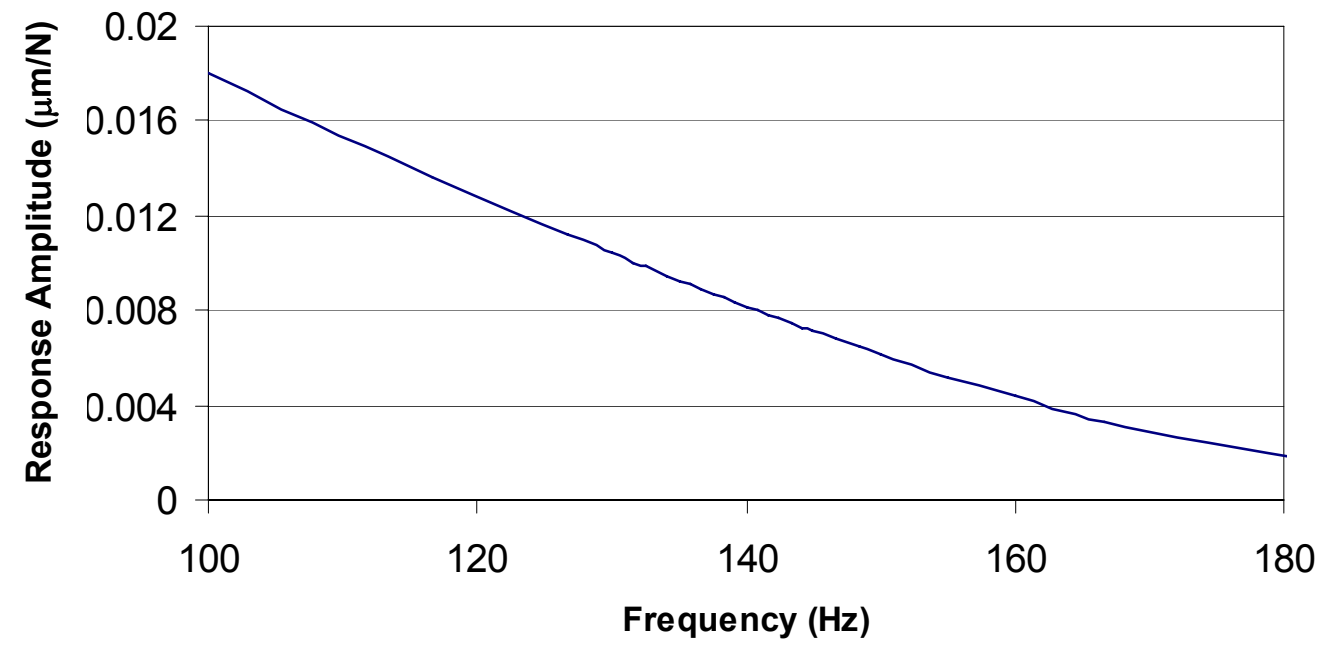

Fig. 18 Two-bearing response at MB1 to force at MB2 force 


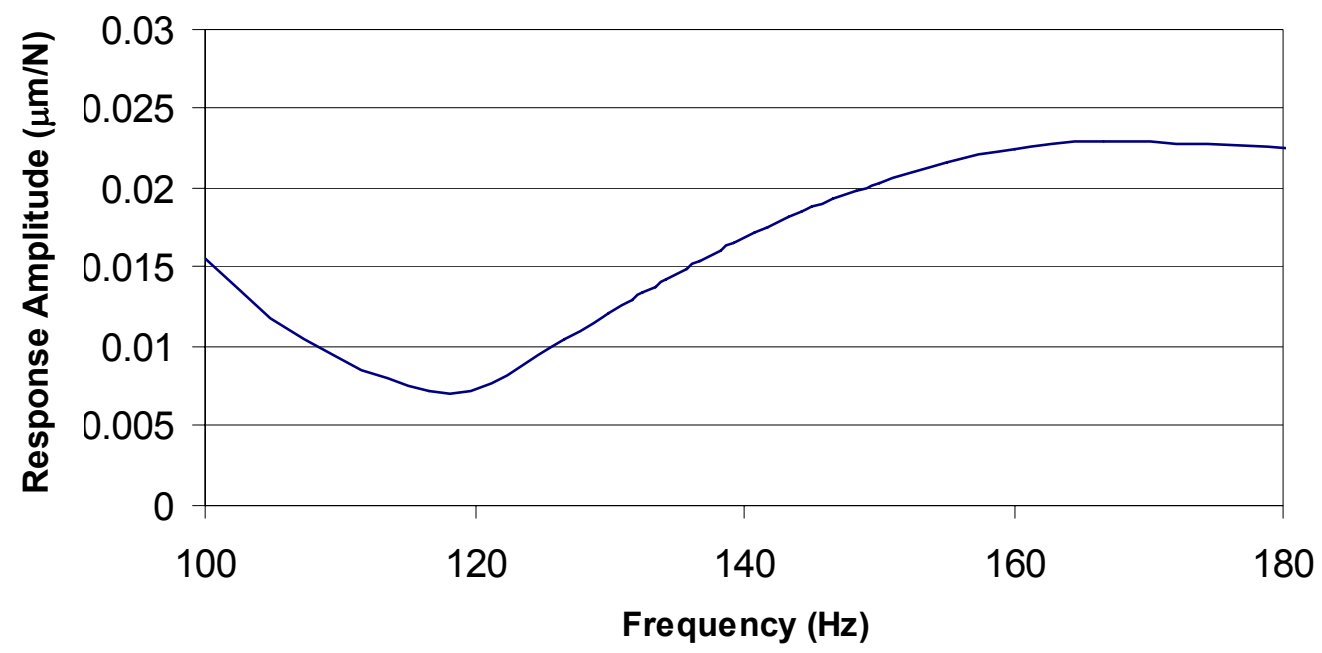

Fig. 19 Two-bearing response at MB2 to force at MB2 force

\section{Drive-End Bearing System Model}

The next system model (Fig. 20) to be considered includes only the drive-end bearing. This model is a candidate for the test case where measurements are taken only at the non-drive bearing. Fig. 21 shows the response of the non-drive MB to a force applied at the non-drive MB. 


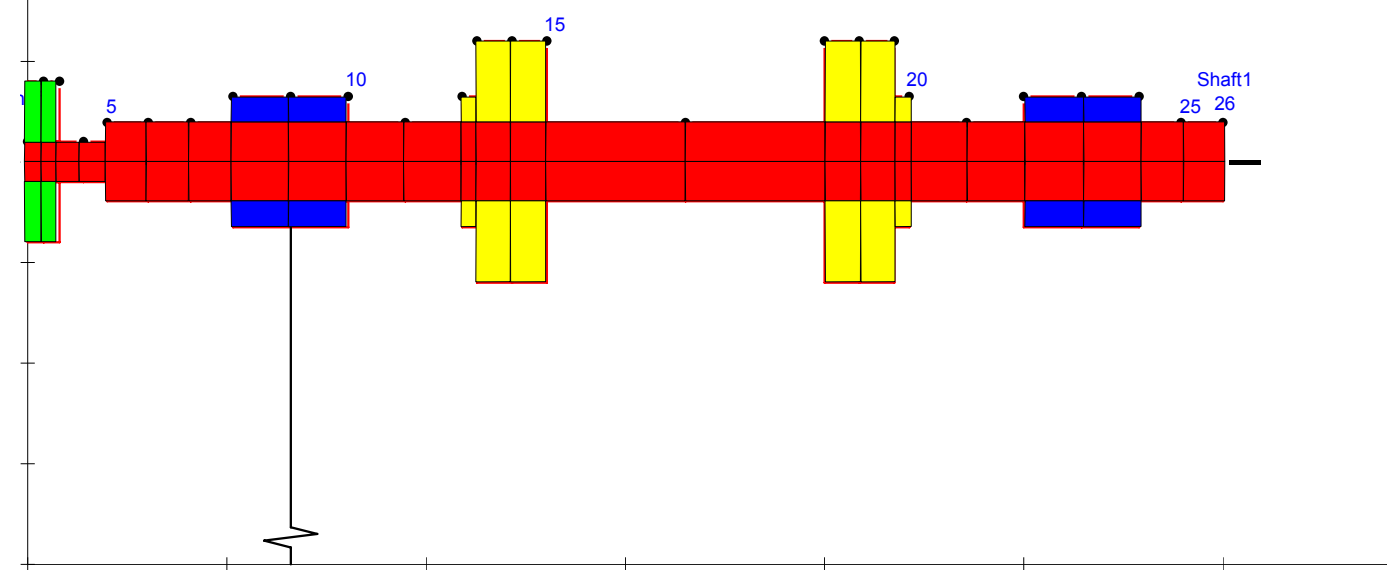

Fig. 20 Rotor-bearing system model with drive-end bearing

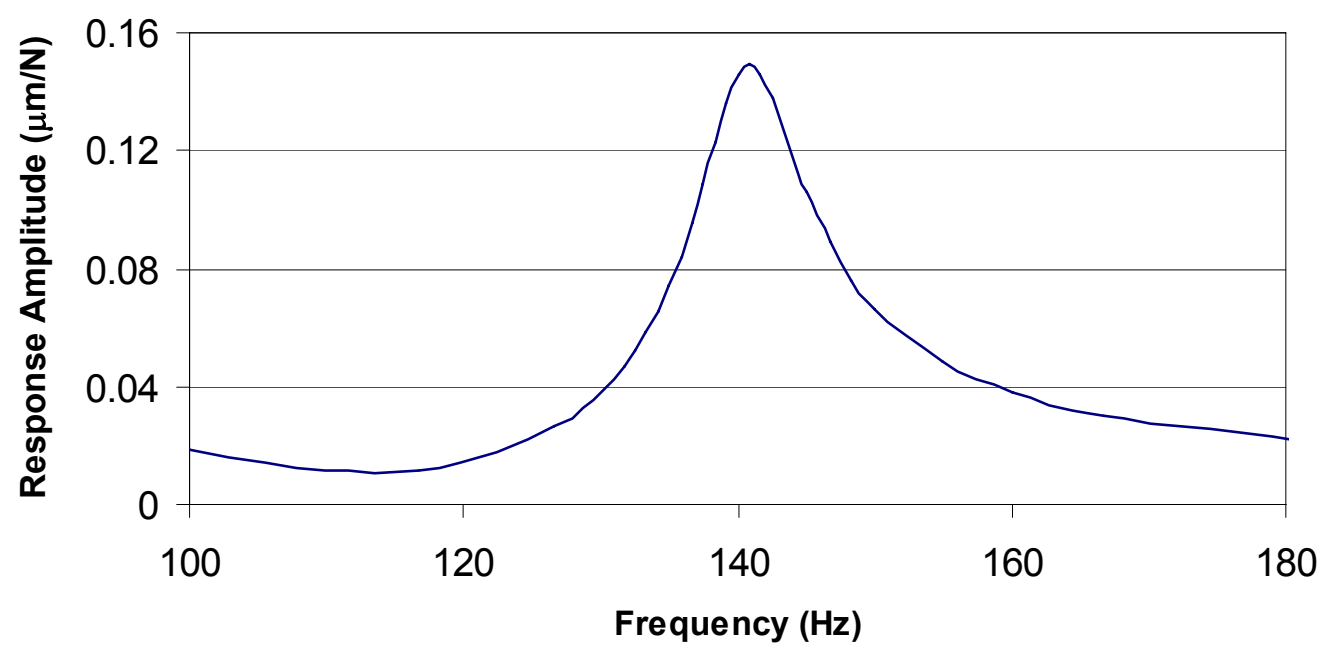

Fig. 21 Drive-end response at MB2 to force at MB2 force 


\section{Non-Drive Bearing System Model}

Fig. 22 displays the model with the non-drive bearing only. This model is a candidate for the test case where measurements are taken only at the drive bearing. Fig. 23 displays the response.

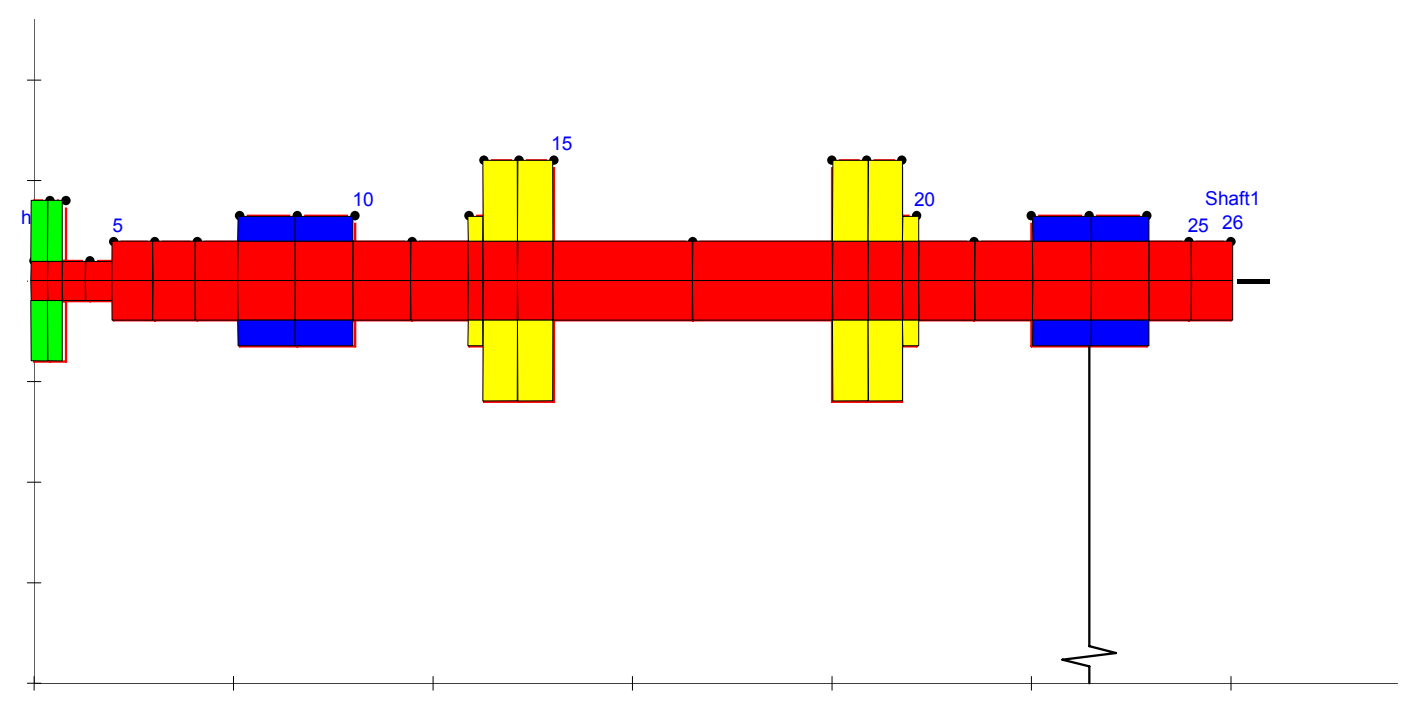

Fig. 22 Rotor-bearing system model with non-drive bearing

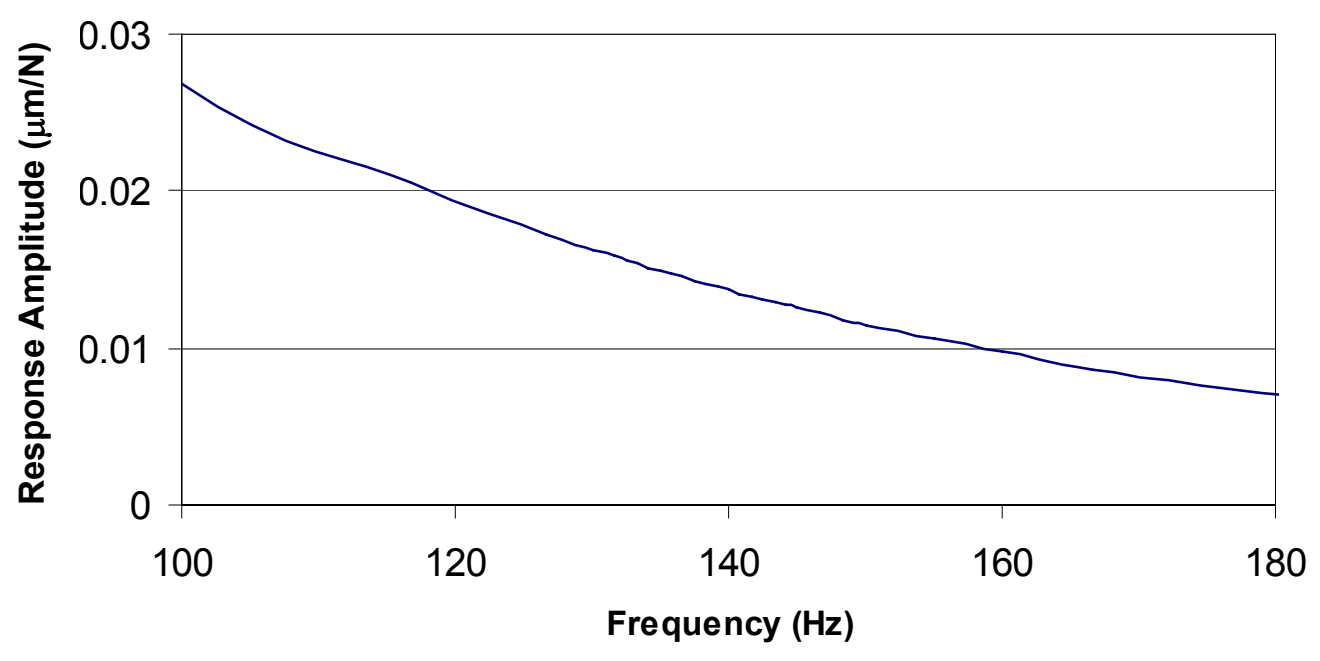

Fig. 23 Non-drive response at MB1 to force at MB1 force 


\section{Free-Free System Model}

The final system model does not include either bearing. Forces are applied at both bearing locations and responses are predicted. Fig. 24 shows the system model, and Fig. 25 through Fig. 28 display the response characteristics.

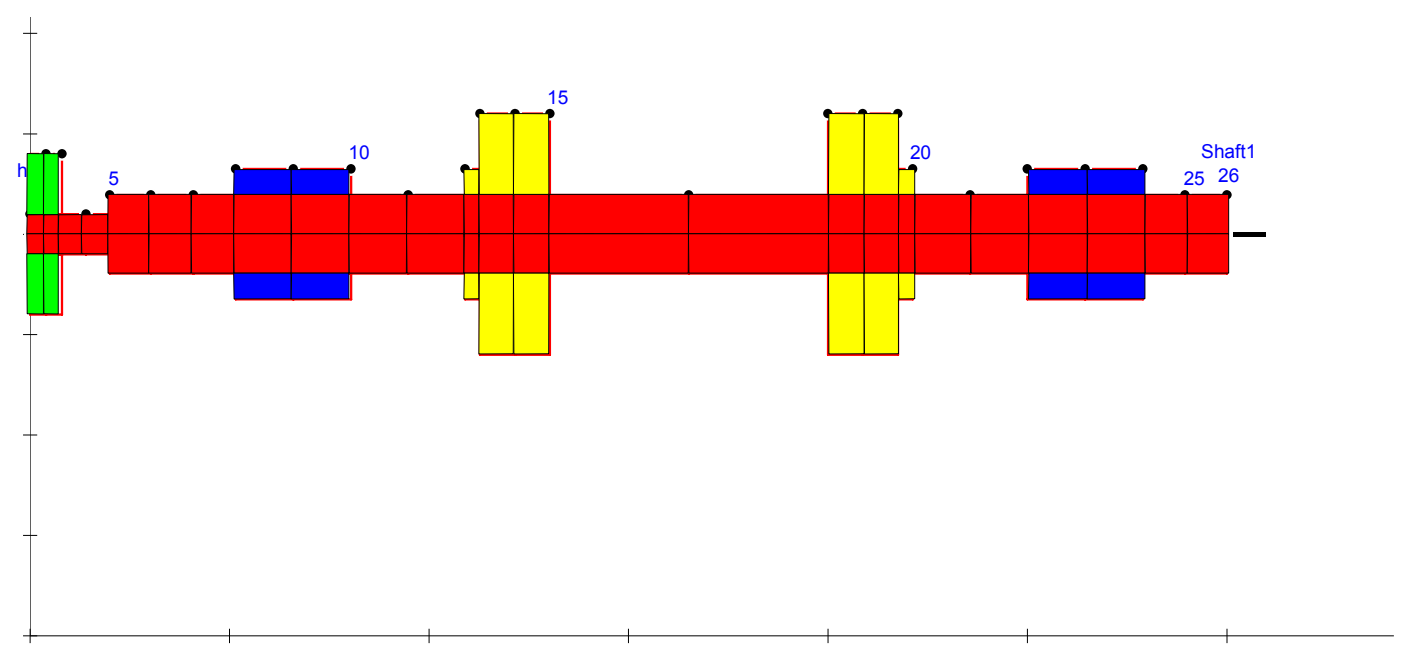

Fig. 24 Rotor-bearing system model with no bearings

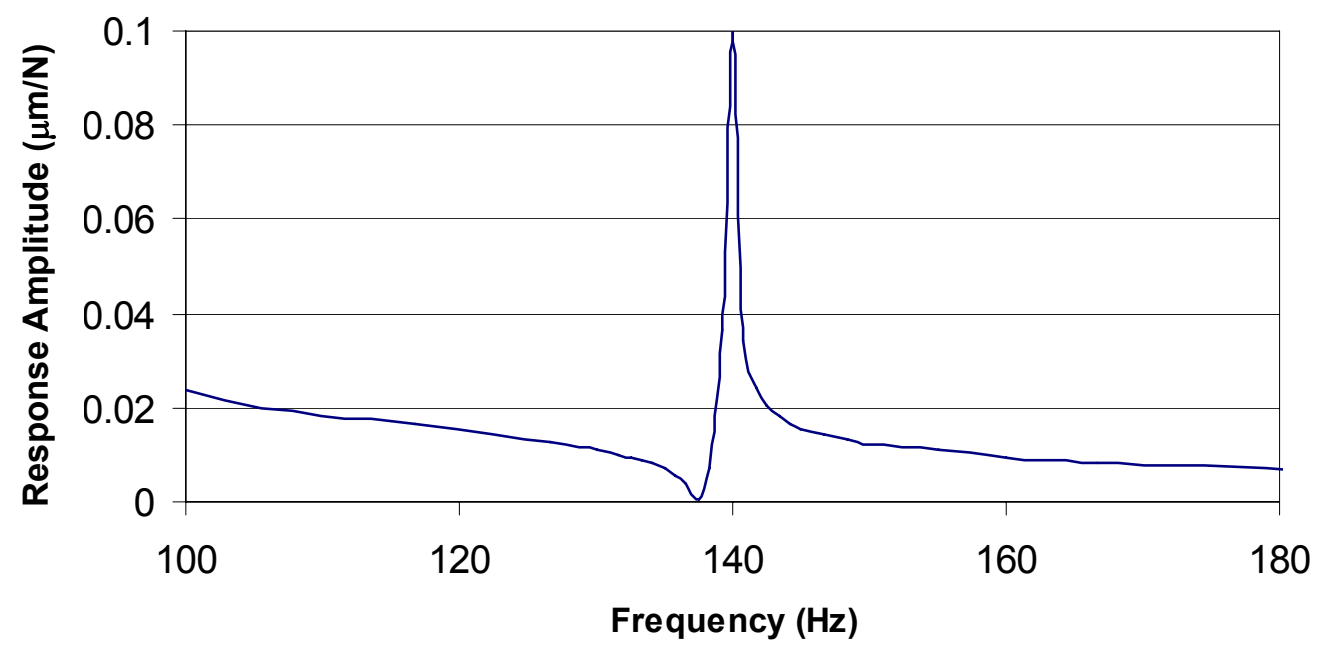

Fig. 25 Response at MB1 to force at MB1 force 


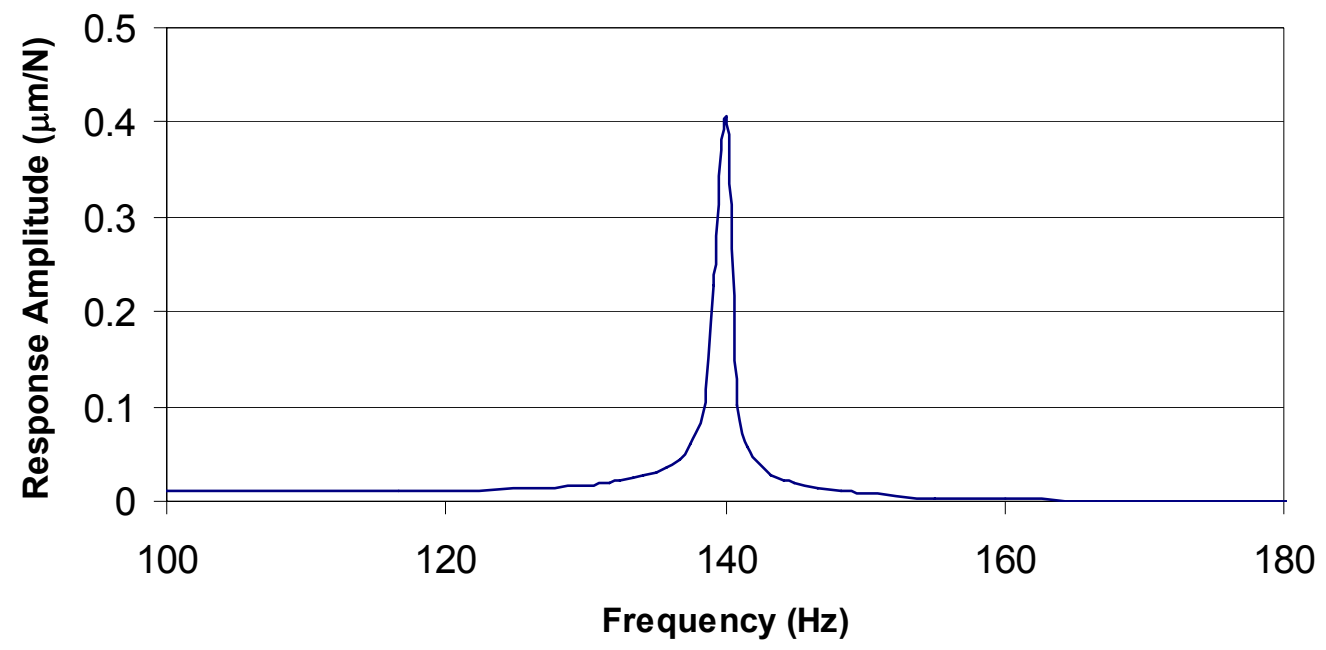

Fig. 26 Response at MB2 to force at MB1 force

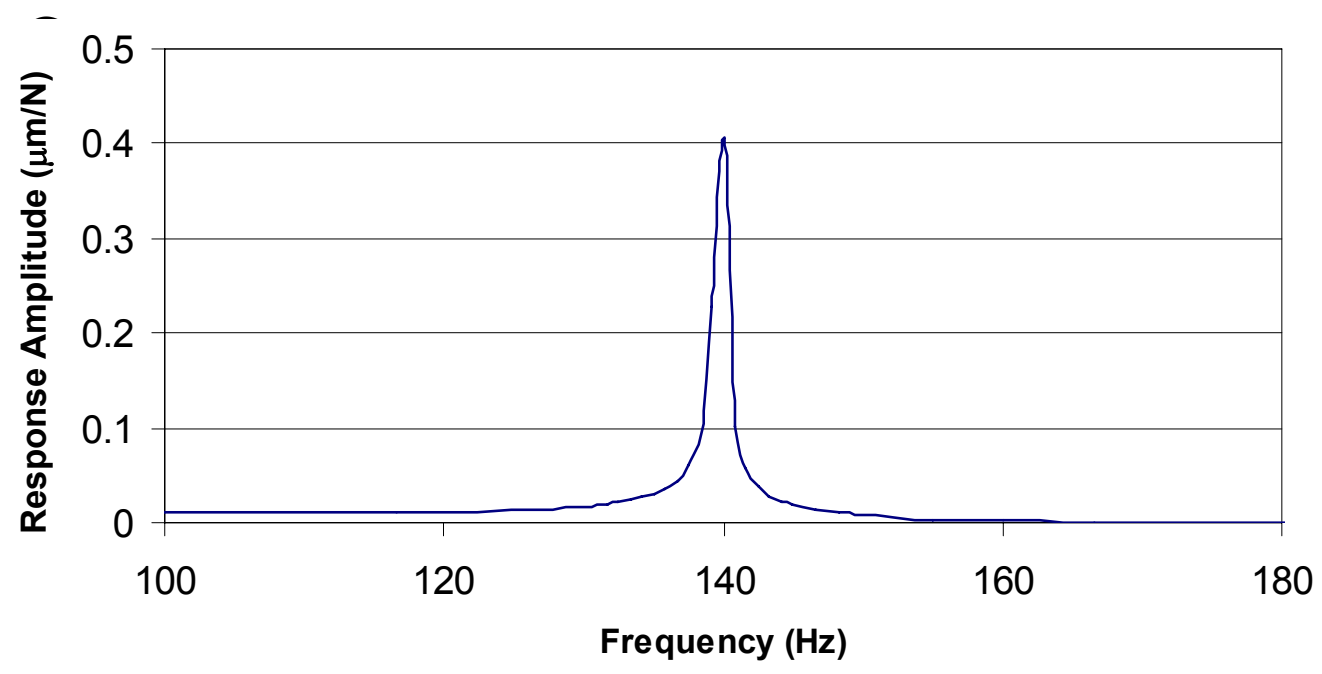

Fig. 27 Response at MB1 to force at MB2 force 


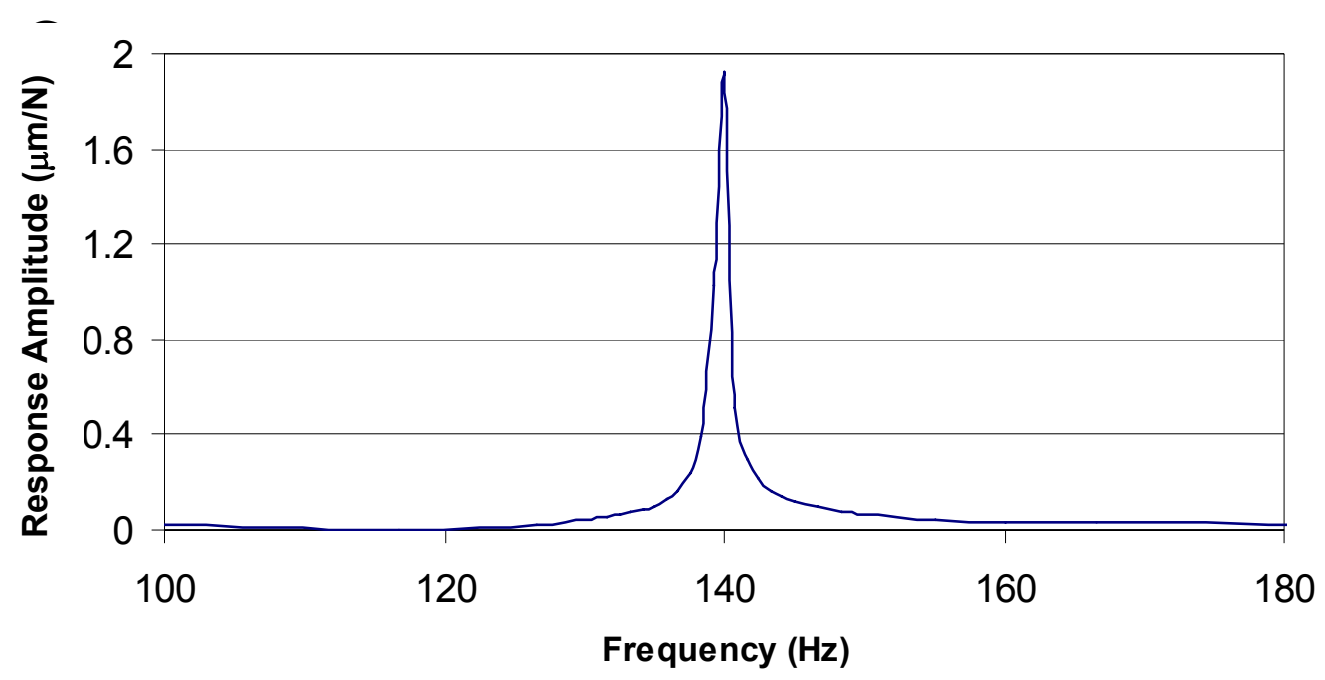

Fig. 28 Response at MB2 to force at MB2 force

\section{Dynamic Flexibility Transfer Functions}

To compare the response predictions from $X L T R C^{2}$ to experimental results, dynamic flexibility transfer functions (DFTFs) need to be discussed. These are simply frequency response functions that capture the position response of the system at specific locations to applied forces. The forces are the DFTF inputs, and the positions are the DFTF outputs. The input forces and output positions need not be at the same physical locations. The DFTFs characterize the multi-input multi-output (MIMO) behavior of the system.

For the present research, the input force is applied by the MB poles and the positions are measured with the motion probes installed in the MBs. This provides two inputs and two outputs for each bearing. DFTFs can be computed using the measurements from one MB, resulting in system model with 2 inputs and 2 outputs. The DFTFs can also be computed using the measurements from both bearings, with a system model of 4 inputs 
and 4 outputs. Experimental computation of DFTFs is addressed in this section and also in references [8,17]. The experimental DFTFs can be compared with the forced response predictions from $X L T R C^{2}$ to determine the appropriate model for an MB acting as a calibrated exciter. The experimental DFTFs also play an important role in the frequency domain parameter identification.

\section{Single Bearing Experimental DFTFs}

Single bearing experimental DFTFs are computed by considering separately the forces and response measured at each bearing. The single bearing DFTFs are experimentally determined by exciting the system at the MB with two linearly independent excitations at each test frequency. Equations (6) describe the mathematical context of the of the DFTF matrix G. Forces $f_{x}$ and $f_{y}$ represent the amplitude and phase (complex values) of the force applied to the rotor by the MB in the horizontal and vertical directions respectively. Positions $x$ and $y$ represent the amplitude and phase of the rotor response at the $\mathrm{MB}$ in the horizontal and vertical directions respectively.

$$
\left[\begin{array}{l}
x \\
y
\end{array}\right]=\left[\begin{array}{ll}
G_{x x}(\omega) & G_{y x}(\omega) \\
G_{x y}(\omega) & G_{y y}(\omega)
\end{array}\right]\left[\begin{array}{l}
f_{x} \\
f_{y}
\end{array}\right]
$$

The transfer function values are computed at each test frequency using the vectors of

position and force measurements from two linearly independent excitations. Position vectors $X_{1}$ and $X_{2}$ and force vectors $F_{1}$ and $F_{2}$ contain the amplitude and phase information from the two excitations.

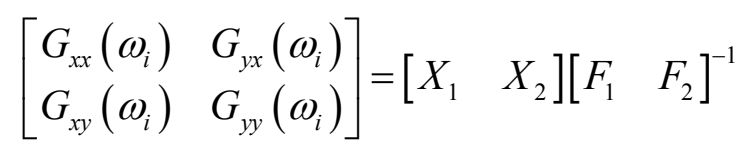

The system can be excited at the desired frequencies sequentially (in separate tests) or simultaneous. In either case a fast Fourier transform (FFT) is performed to obtain the amplitude and phase information from the test measurements. The above calculation is 
performed using the information for each test frequency developing an experimental transfer function. The result is an amplitude and phase map of the position response to the input forces vs. excitation frequency.

In practice, there are numerical difficulties with computing transfer functions this way. For example, the force levels at or near a system natural frequency tend to be very low. This typically results in a poor signal-to-noise ratio for the force measurements at these frequencies. That reduces the accuracy of the force matrix inversion.

Other approaches to computing transfer functions use spectral analysis. The built-in Matlab function spa is commonly used for transfer function computation. This function allows the user to specify a window lag size. This has the effect of smoothing the transfer functions, but also increases the apparent damping near natural frequencies. There is a trade off between reducing the noise in the transfer function and over predicting damping. Fortunately, the measurements taken for this research project have high sensitivity and low noise, and very little, if any, smoothing is required.

For the purpose of MB model validation, the diagonal terms of the DFTF matrix are of primary interest. The system is not rotating and is reasonably symmetric, therefore the off diagonal terms are expected to be negligible. The FE model from $X L T R C^{2}$ assumes the bearings are symmetric and neglects support structure flexibility. For this reason, there will likely be some discrepancy between the experimental results and the predictions. The results below demonstrate that the modeling assumptions are sufficient for the present analysis.

Below are the amplitudes (Fig. 29) and phases (Fig. 30) of the single bearing experimental DFTFs. The upper left displays the drive-end bearing results (MB1), and the lower right displays the non-drive bearing results (MB2). The subplot locations correspond to the locations of the terms displayed in the DFTF matrices above. The 
solid line indicates the amplitude and phase values; the dots represent the uncertainties determined using uncertainties from repeated tests.

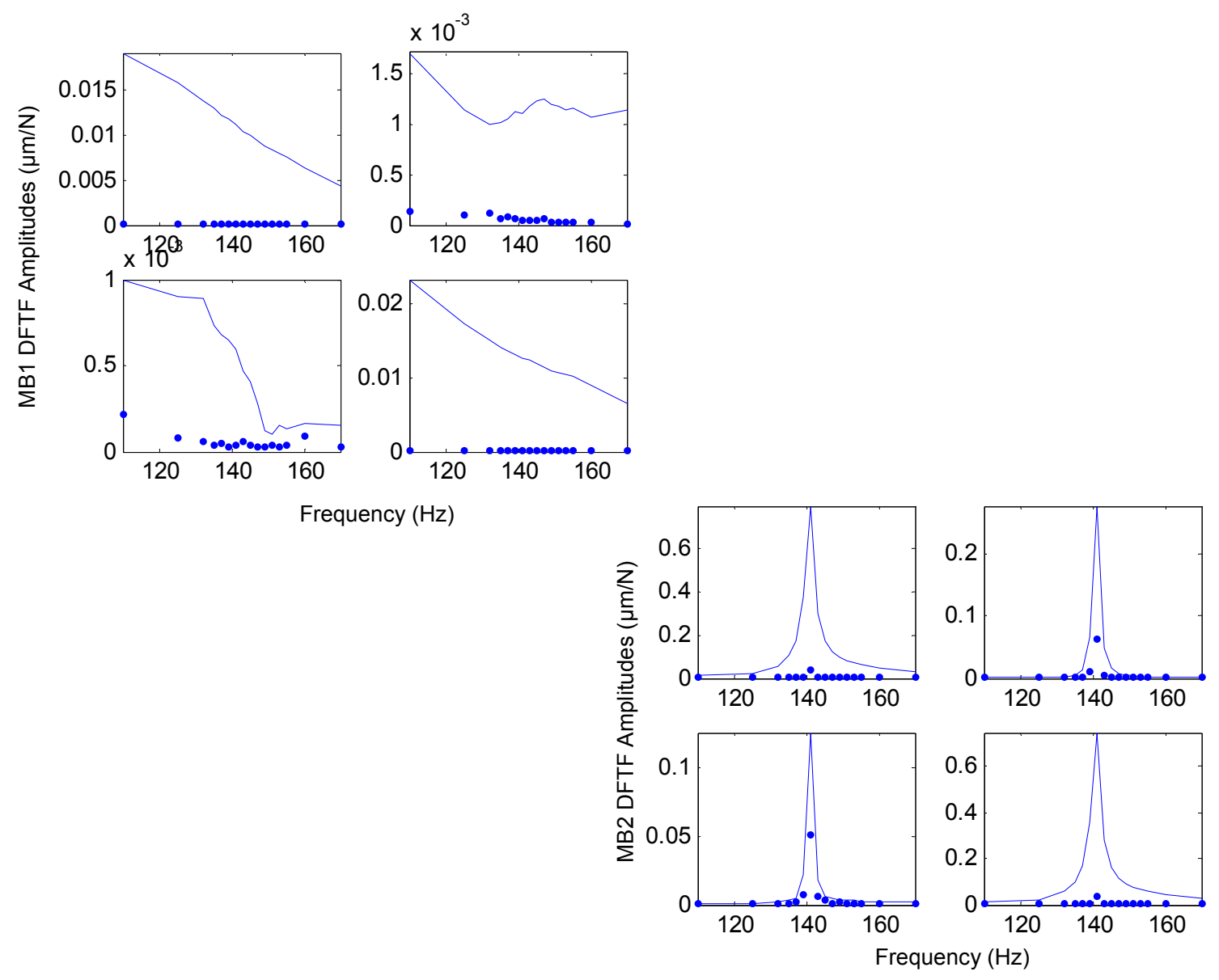

Fig. 29 Single bearing experimental DFTF amplitudes 

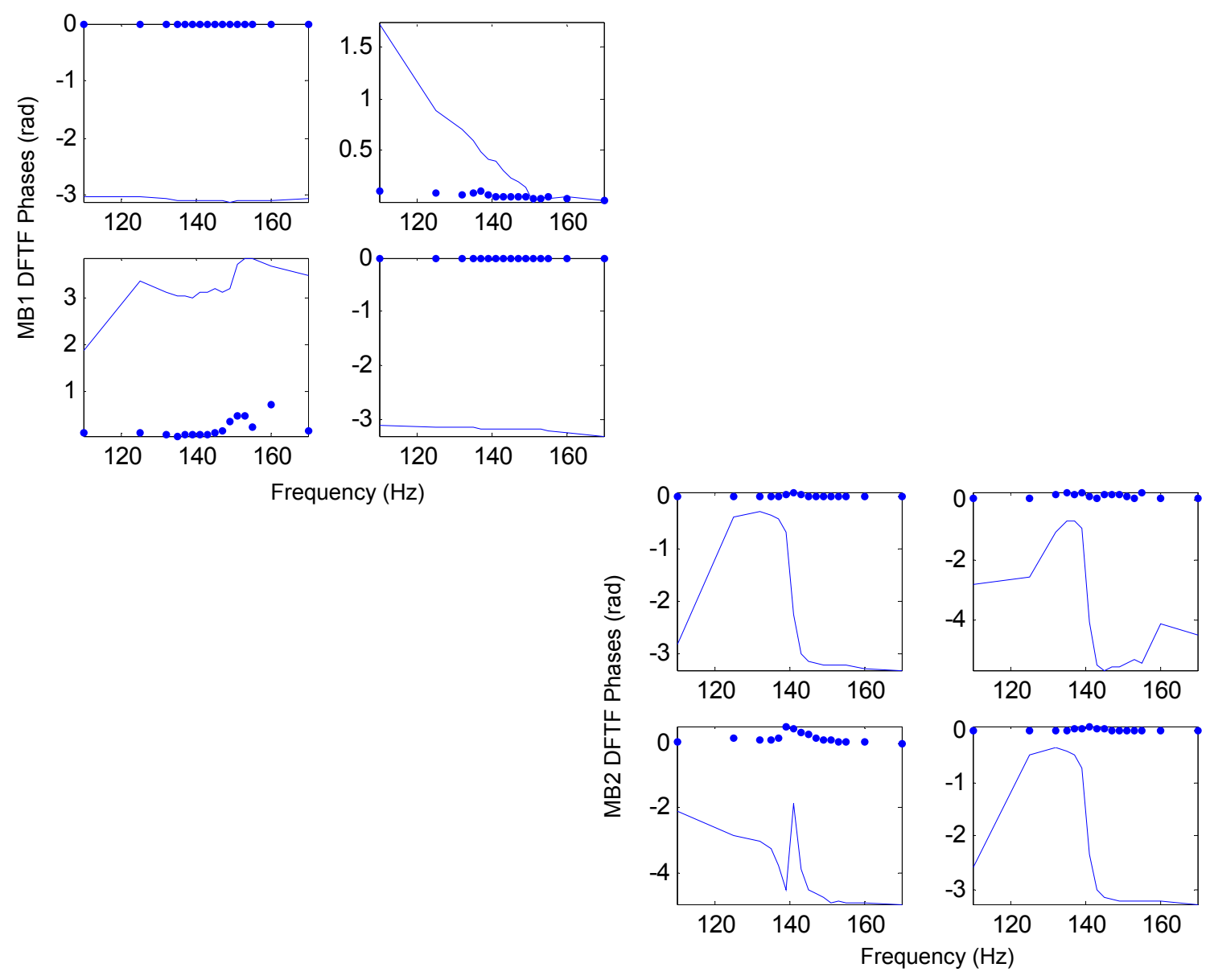

Fig. 30 Single bearing DFTF phases

Results of these tests are comparable to those of previous tests. Uncertainties are displayed as dots relative to the zero point on the y-axis. Uncertainties tend to be high in areas of high flexibility because of low force levels.

\section{Two Bearing Experimental DFTFs}

Two bearing experimental DFTFs are computed by shaking the rotor in the horizontal and vertical directions at each bearing and recording forces at each bearing. This requires four linearly independent excitations. The DFTF matrix and its notations are displayed in Eq. (8). The DFTF matrix $\mathbf{G}$ can again be computed by inverting the matrix 
of force vectors as shown in Eq. (9). However, spectral analysis is preferred because the excitation frequencies are centered about a natural frequency.

$$
\begin{gathered}
{\left[\begin{array}{l}
x_{M B 1} \\
y_{M B 1} \\
x_{M B 2} \\
y_{M B 2}
\end{array}\right]=\left[\begin{array}{llll}
G_{11} & G_{12} & G_{13} & G_{14} \\
G_{21} & G_{22} & G_{23} & G_{24} \\
G_{31} & G_{32} & G_{33} & G_{34} \\
G_{41} & G_{42} & G_{43} & G_{44}
\end{array}\right]\left[\begin{array}{l}
F_{x}^{M B 1} \\
F_{y}{ }^{M B 1} \\
F_{x}{ }^{M B 2} \\
F_{y}{ }^{M B 2}
\end{array}\right]} \\
\mathbf{G}\left(\omega_{i}\right)=\left[\begin{array}{llll}
X_{1} & X_{2} & X_{3} & X_{4}
\end{array}\right]\left[\begin{array}{llll}
F_{1} & F_{2} & F_{3} & F_{4}
\end{array}\right]^{-1}
\end{gathered}
$$

Fig. 31 contains the two bearing DFTF amplitudes, and Fig. 32 shows the DFTF phases. The subplot locations correspond to the elements of the DFTF matrix from Eq. (8).

\section{DFTF Experiment vs. Theory}

The $X L T R C^{2}$ predictions and the experimental results provide a basis for comparison and suggest appropriate system models for each DFTF. The similarities and discrepancies of results provide a motivation for further analysis of the rotor-bearing system. The results and comparisons lead to conclusions about the appropriate system interaction model for magnetic bearings acting as calibrated exciters.

\section{Comparison}

Comparing the $X L T R C^{2}$ predictions to the experimental results reveals the appropriate system model for each of the experimental DFTFs. First, the predictions for the two bearing system (Fig. 15) do not correlate with two-bearing experimental results. In addition to the discrepancies in the shapes of the DFTFs, the amplitudes are an order of magnitude smaller than most of the experimental results. 

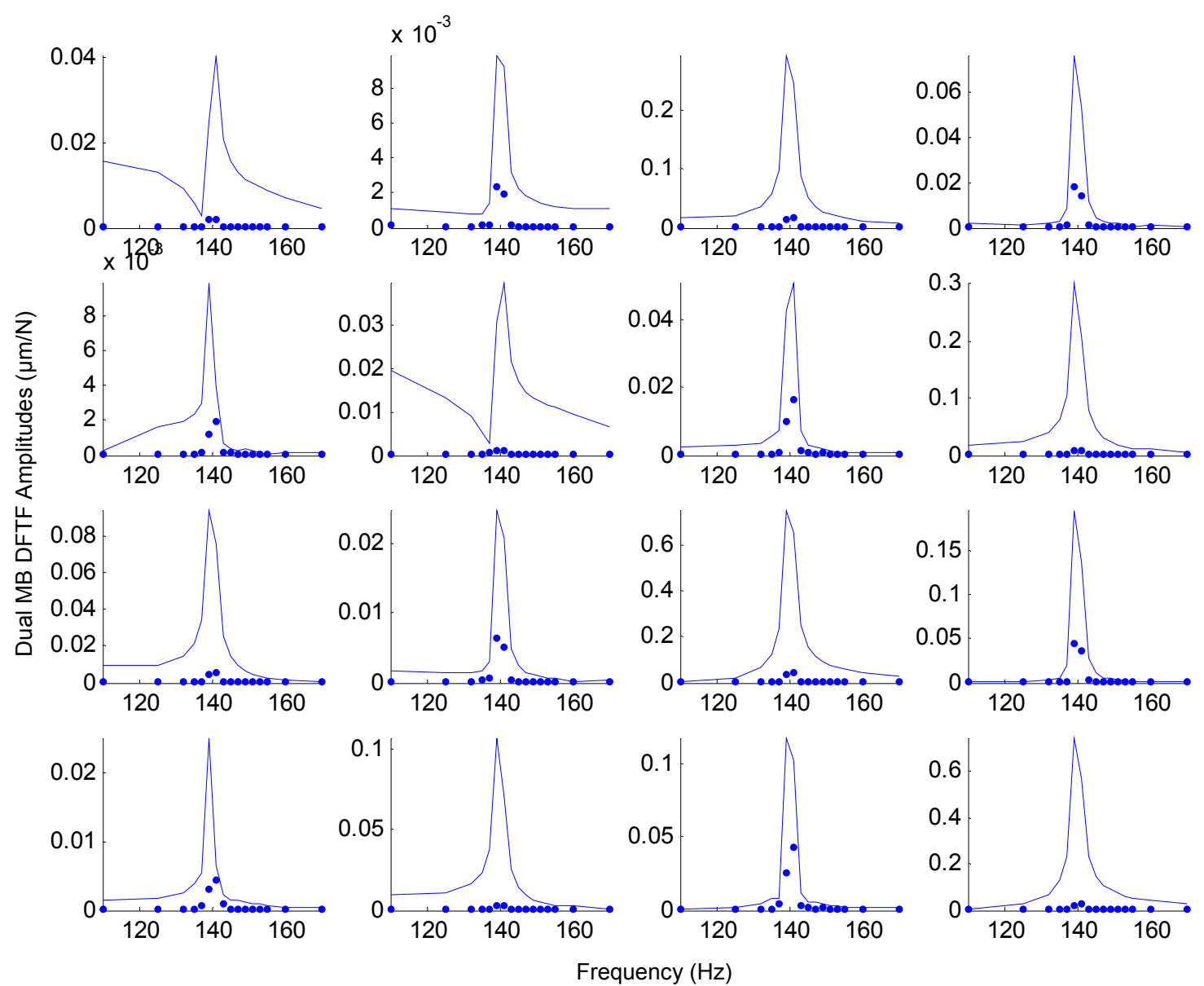

Fig. 31 Two bearing DFTF amplitudes 

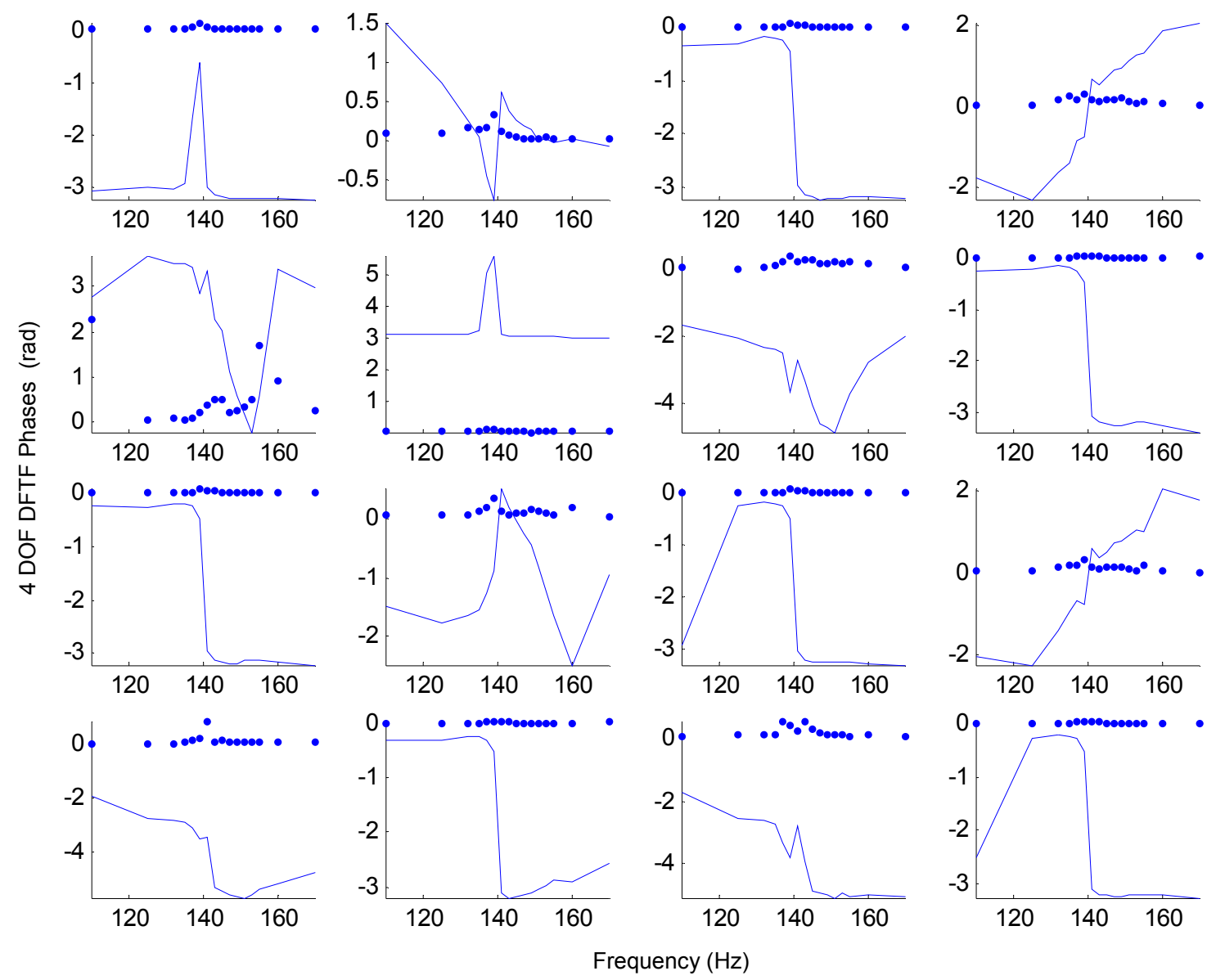

Fig. 32 Two bearing DFTF phases

The drive-end MB system model (Fig. 20) results display some agreement with the experimental single bearing DFTF results at the non-drive bearing. Fig. 33 compares the experimental results to the $X L T R C^{2}$ predictions. The major discrepancy is the prediction of significantly more damping than is found experimentally. The absence of damping can be attributed to the fact that the drive-end bearing has been machined to remove damaged material from the laminate surfaces. This results in an increased air gap and a decreased load capacity. The over-prediction of damping could also be related to a slight error in the predicted mode shape. The reasoning behind the later reason will be explained in the following section. 


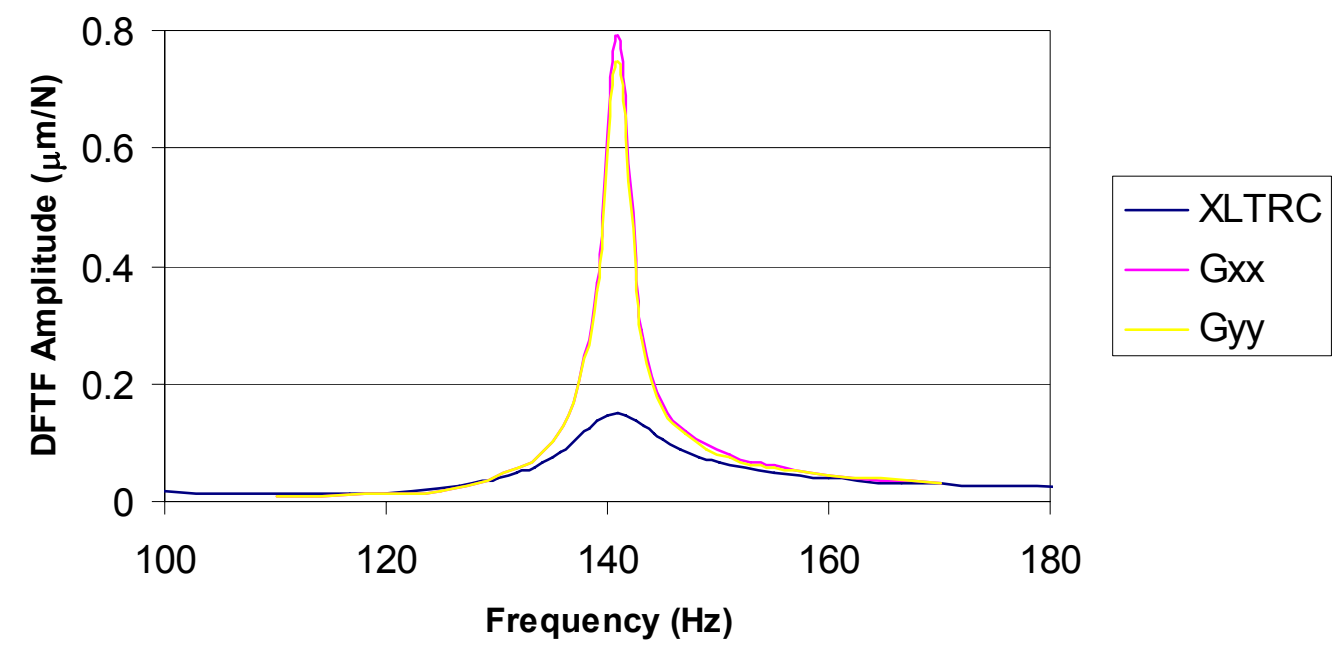

Fig. 33 Single bearing DFTF comparison for the non-drive bearing measurements

The non-drive bearing system model (Fig. 22) displays a strong correlation with the experimental single bearing DFTF results for the drive-end bearing. Reduced agreement of the horizontal transfer function suggests the possible influence of support flexibility on the results. Fig. 34 compares theses results.

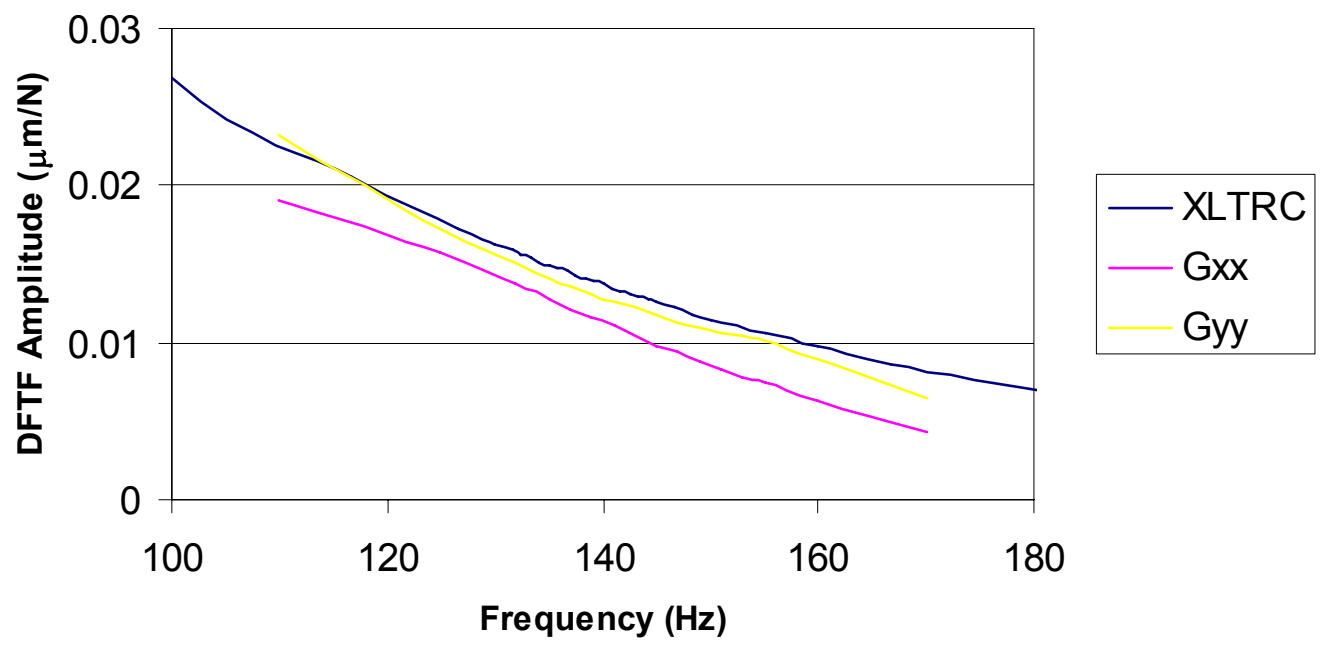

Fig. 34 Single bearing DFTF comparison for the drive-end bearing measurements 
The two bearing experimental results agree with the free-free system model (Fig. 24). The free-free system model does not contain any damping mechanisms. In reality small sources of damping, such as structural damping, exist. In addition, the FOSGs exhibit some position dependence, which produces force measurement errors that are most noticeable in regions of low flexibility. The position dependence of the strain is addressed in [8] and is also addressed later in the FOSG calibration section. The presence of small levels of damping and the force measurement error account for the over prediction of the DFTFs at the natural frequency. Fig. 35 through Fig. 38 compare the experimental and theoretical DFTF amplitudes.

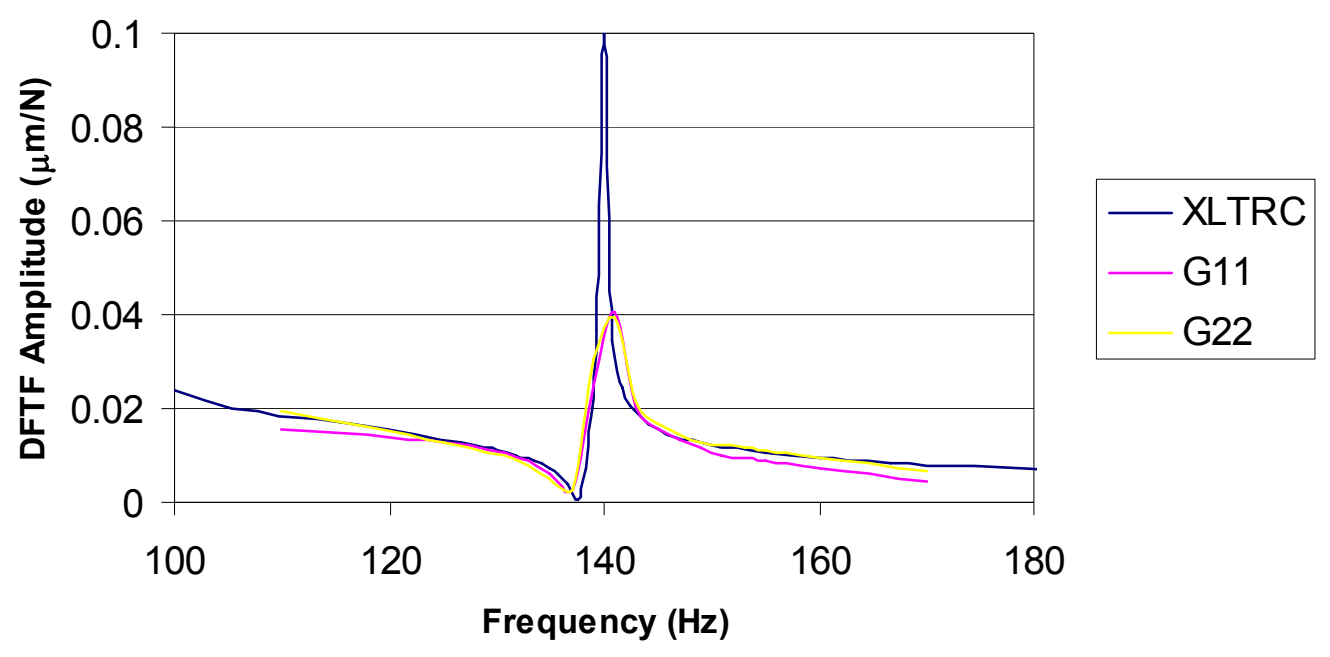

Fig. 354 DOF DFTF comparison for $G_{11}$ and $G_{22}$ 


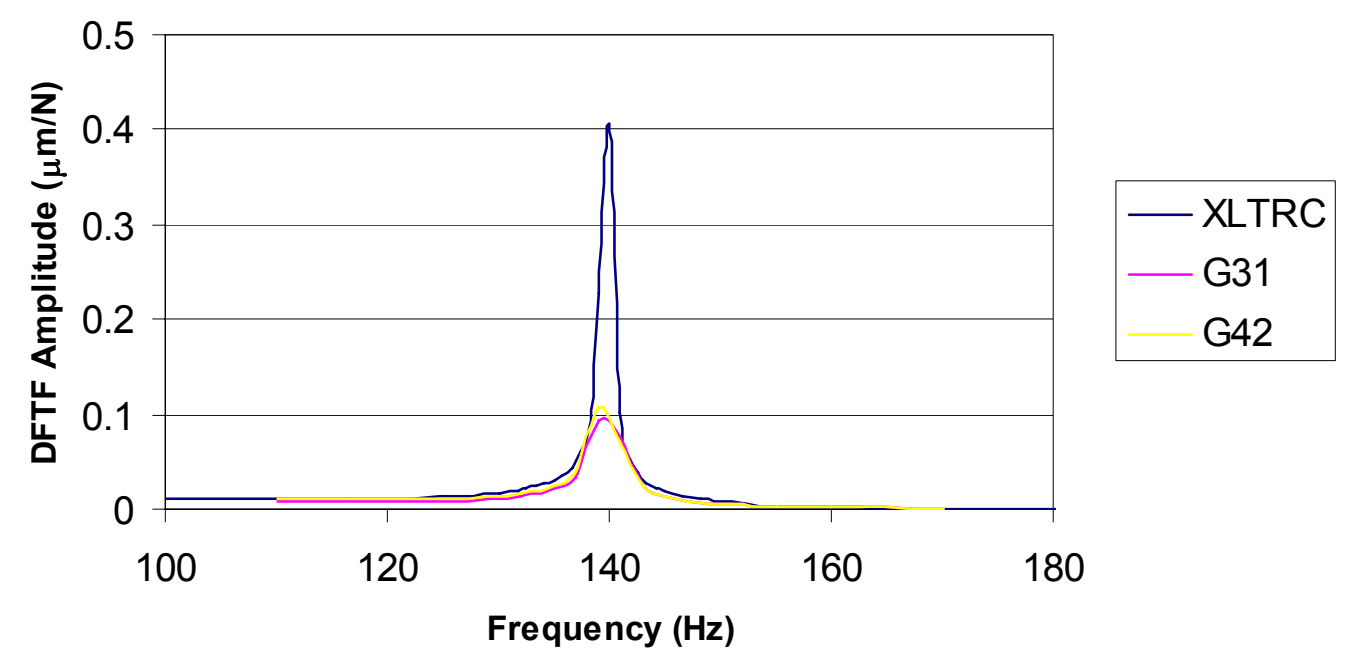

Fig. 364 DOF DFTF comparison for $G_{31}$ and $G_{42}$

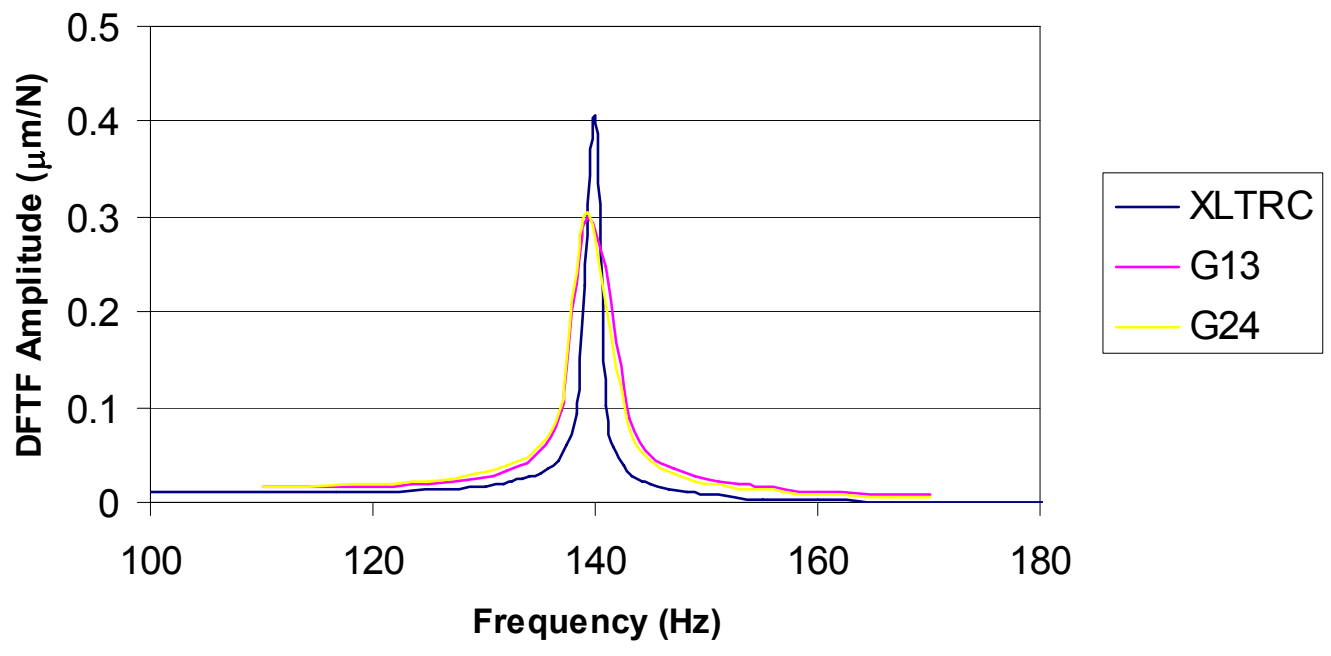

Fig. 374 DOF DFTF comparison for $G_{13}$ and $G_{24}$ 


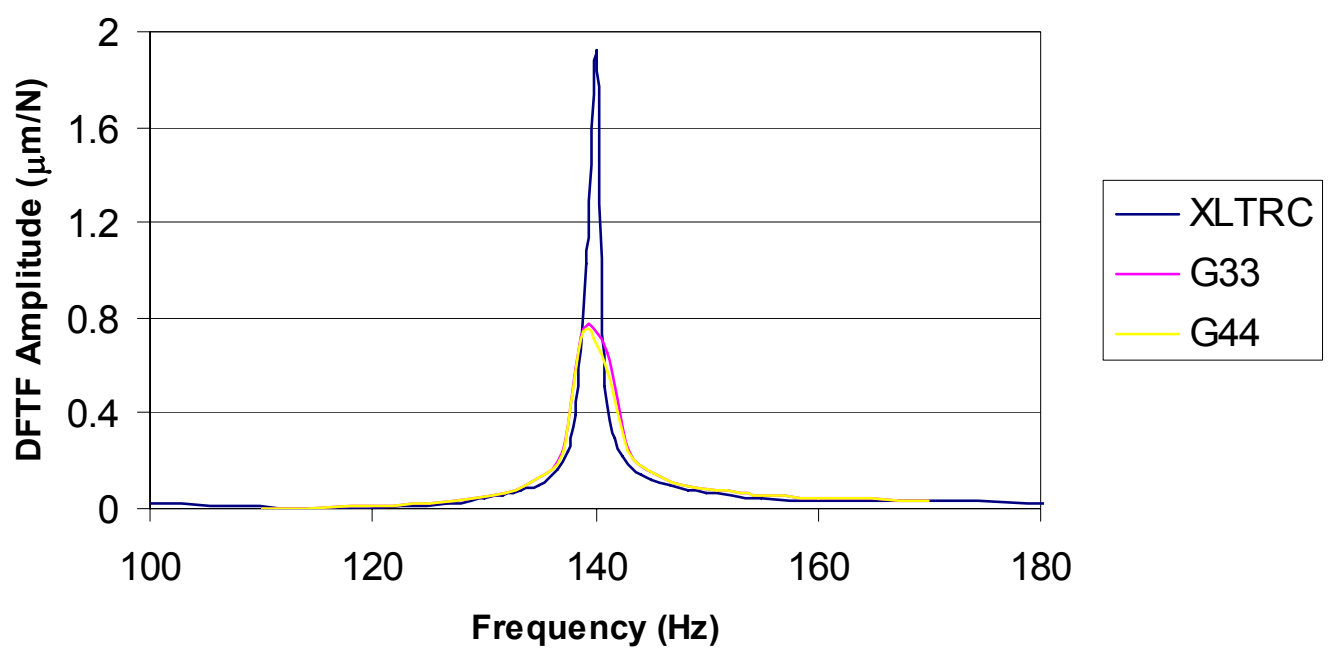

Fig. 384 DOF DFTF comparison for $G_{33}$ and $G_{44}$

The differences between experiment and theory are addressed in due course. The following section addresses issues with the first comparison (non-drive bearing measurements), and later sections include further refinement of the rotor model, resulting in excellent experiment to theory correlation. It is important to draw two conclusions from these comparisons: (i) MB(s) acting as calibrated exciters (MBs where force and position measurements are taken) are excluded from the experimental system model (ii) the hypothesis that the $\mathrm{MB}(\mathrm{s})$ acting as calibrated exciters are included in the system model is clearly refuted. The latter conclusion is, perhaps, more convincing. For verification of this conclusion compare the two bearing predictions (Fig. 16 through Fig. 19) to the two bearing experimental DFTFs (Fig. 31 and Fig. 32).

\section{Modal Analysis}

Closer examination of the rotor model can explain some of the properties of the DFTFs and some of the discrepancies between theoretical and experimental results. The first mode shape of the free-free rotor system is shown in Fig. 39. 


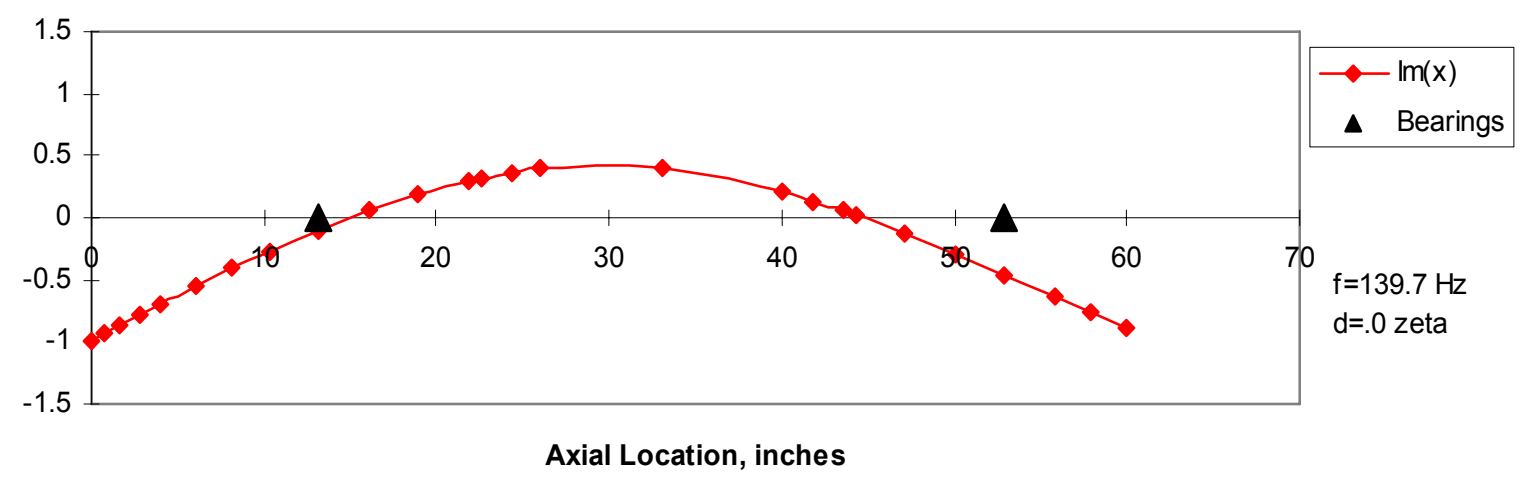

Fig. 39 First damped Eigenvalue mode shape

The most significant revelation of this plot is the impact of the bearing locations. The drive-end bearing is located very near to one of the nodes of the mode shape. This means that an excitation at this location will produce only a slight excitation of the first mode. The location of the non-drive bearing will allow the bearing to damp out the first mode excitation from the other bearing.

Conversely an excitation at the non-drive bearing will excite the first mode, and the drive-end bearing will only be capable of producing slight damping of the first mode. The bearing locations can also contribute to discrepancies between experimental results and predictions. A slight error in the drive-end bearing location or the rotor model can produce a substantial error in predictions for the case with measurements at the nondrive bearing. The over-prediction of damping for the non-drive DFTF may be related to the bearing-node relative position.

The modal analysis could be tested further by attempting to excite higher modes of the rotor using the drive-end bearing. Fig. 40 demonstrates that excitation of the second mode shape is possible using the drive-end bearing; however, the non-drive bearing can damp this mode. The third mode (Fig. 41) can be excited by the drive-end bearing, and only limit damping can be introduced by the non-drive bearing. 


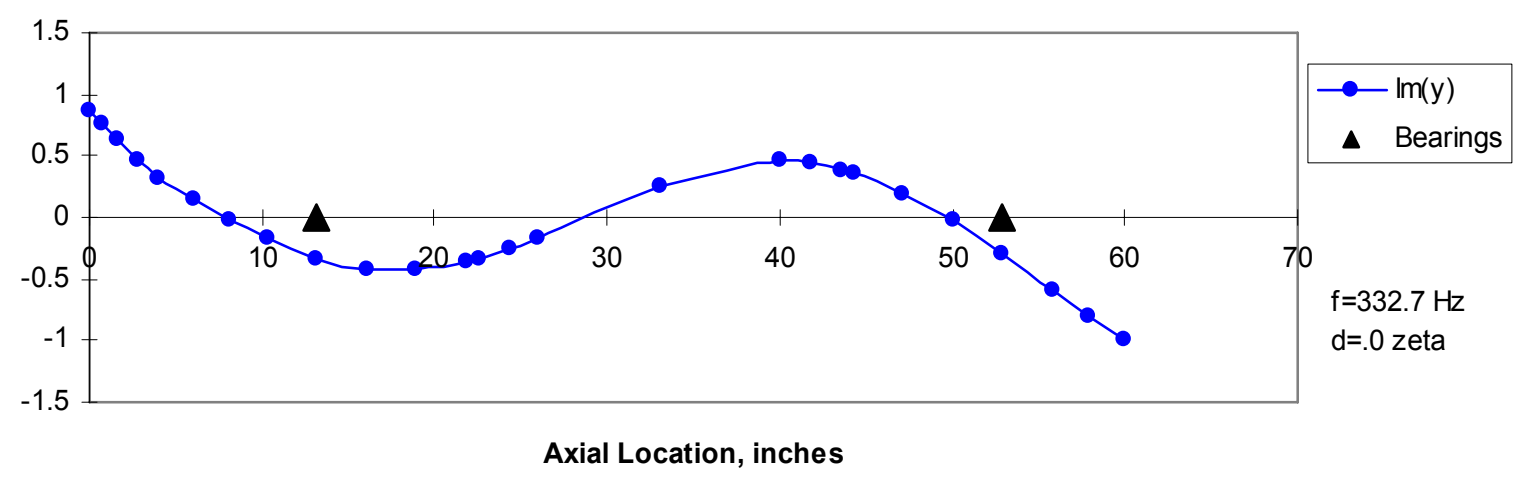

Fig. 40 Second damped Eigenvalue mode shape

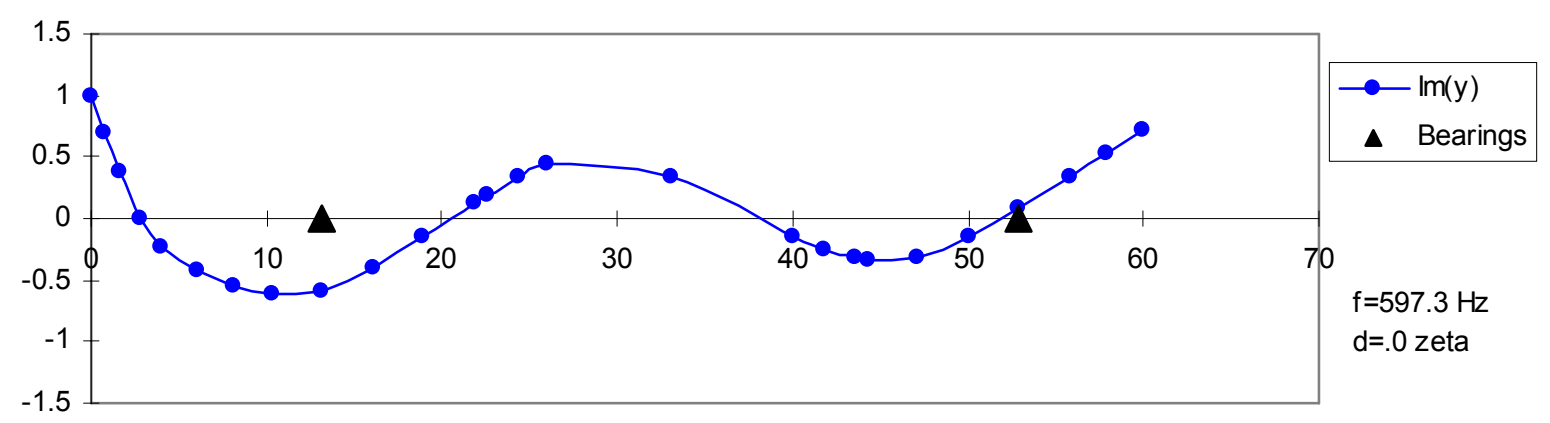

Axial Location, inches

Fig. 41 Third damped Eigenvalue mode shape

\section{Conclusions}

The relevant system models have been reasonably determined for the DFTF experimental tests. The prediction errors can by explained by model factors such as bearing and node locations and experimental factors such as structural damping, force measurement error, and the effects of laminate machining,.

These results indicate that the appropriate model for a magnetic bearing used as a calibrate exciter is simply a known external force. This conclusion is reached because in each of the system models that agree with experimental results, the bearing(s) absent in the system corresponds to the bearing(s) measuring the forces for the DFTFs. 
Fig. 42 shows that the intended excitation force due to the excitation waveform may act in parallel with the representative stiffness and damping characteristics of the bearing. However, the force measurements taken by the FOSGs detect the total force applied to the rotor. The FOSG force measurement is used to compute experimental results, and accordingly, all contributions to the total force acting on the rotor are included in the results.

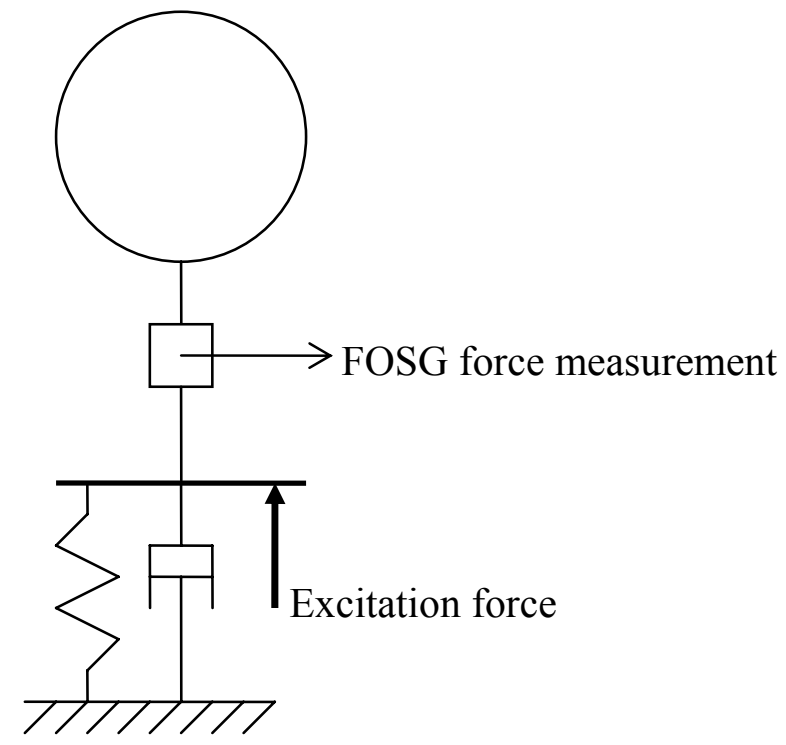

Fig. 42 Model of magnetic bearing as a calibrated exciter

This conclusion is analogous to the physical model for shakers common to other dynamic test rigs. The shaker is hydraulically actuated with either the shaker force or position controlled by a feedback controller. The shaker controller accepts an input waveform used for exciting the shaker. The force measurements for the shaker are taken by a load cell positioned between the test rig and shaker. In this way, the load cell measures the true force applied to the test rig, whether the force is applied as a result of the feedback controller dynamics, the excitation waveform, or both. This analogy 
provides a reasonable illustration of force measurement in a mechanical actuator acting in the same capacity as the MB calibrated exciter.

\section{Rotor Model Validation- Rap Test Results and Comparisons}

The $X L T R C^{2}$ model used in the preceding sections has been tuned to match experimental results from the DFTFs. This model requires additional validation to ensure the accuracy of the model. Performing rap test for various configurations of the rotor can further improve the understanding of the appropriate system model for parameter identification.

\section{Free-Free Rap Test Results}

The first test is performed by suspending the rotor at either end with long (approximately $6 \mathrm{~m}$ ) nylon straps and exciting the rotor with a hammer in the horizontal plane. For this experiment, the coupling is attached to the rotor at one end and a large steel block at the other end. This simulates the condition of the rotor in the test rig when forces are measured (treated as known forces) at both magnetic bearings. The steel block prevents the drive end of the coupling from vibrating significantly.

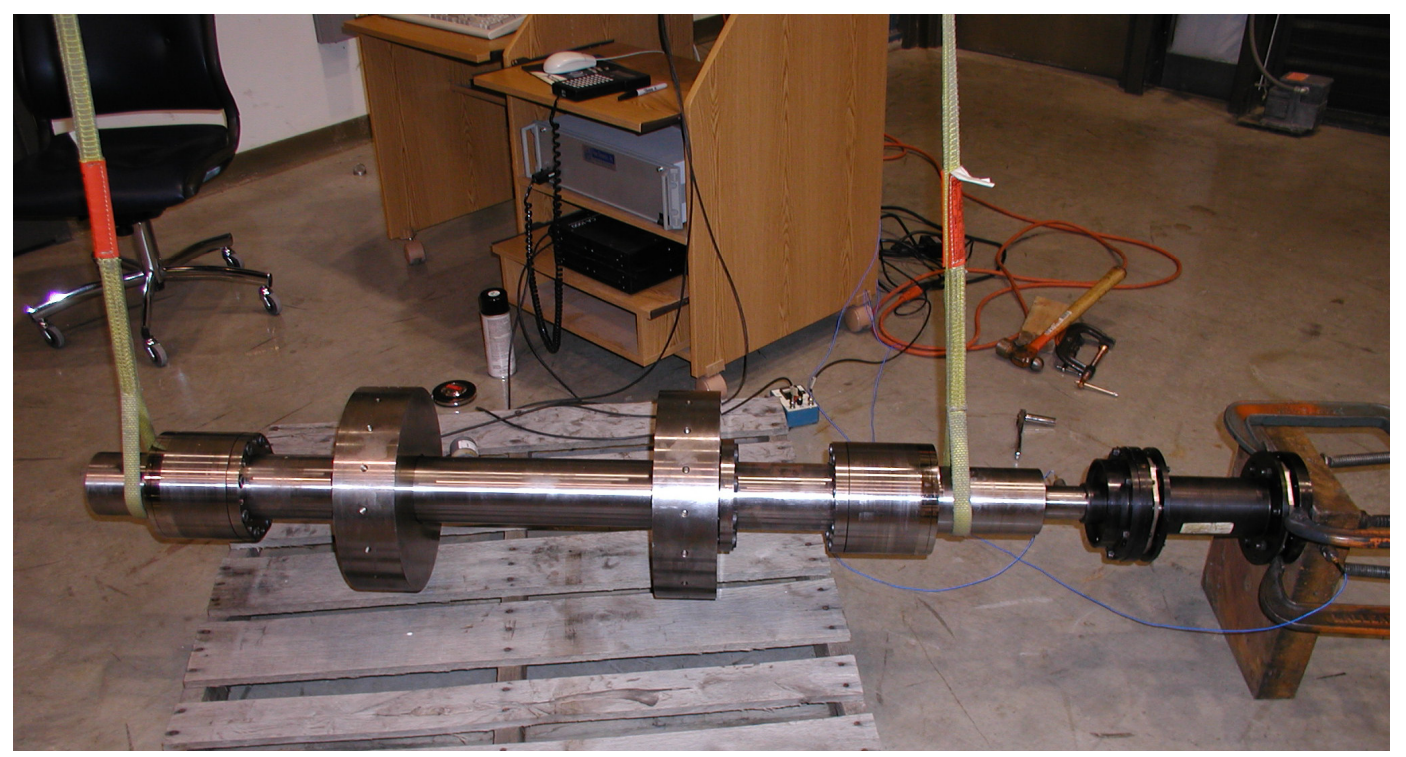

Fig. 43 Free-free rap test 
Fig. 44 displays the first four bending modes of the rotor in above configuration. Fig. 45 compares the first three modes determined experimentally with $X L T R C^{2}$ predictions.

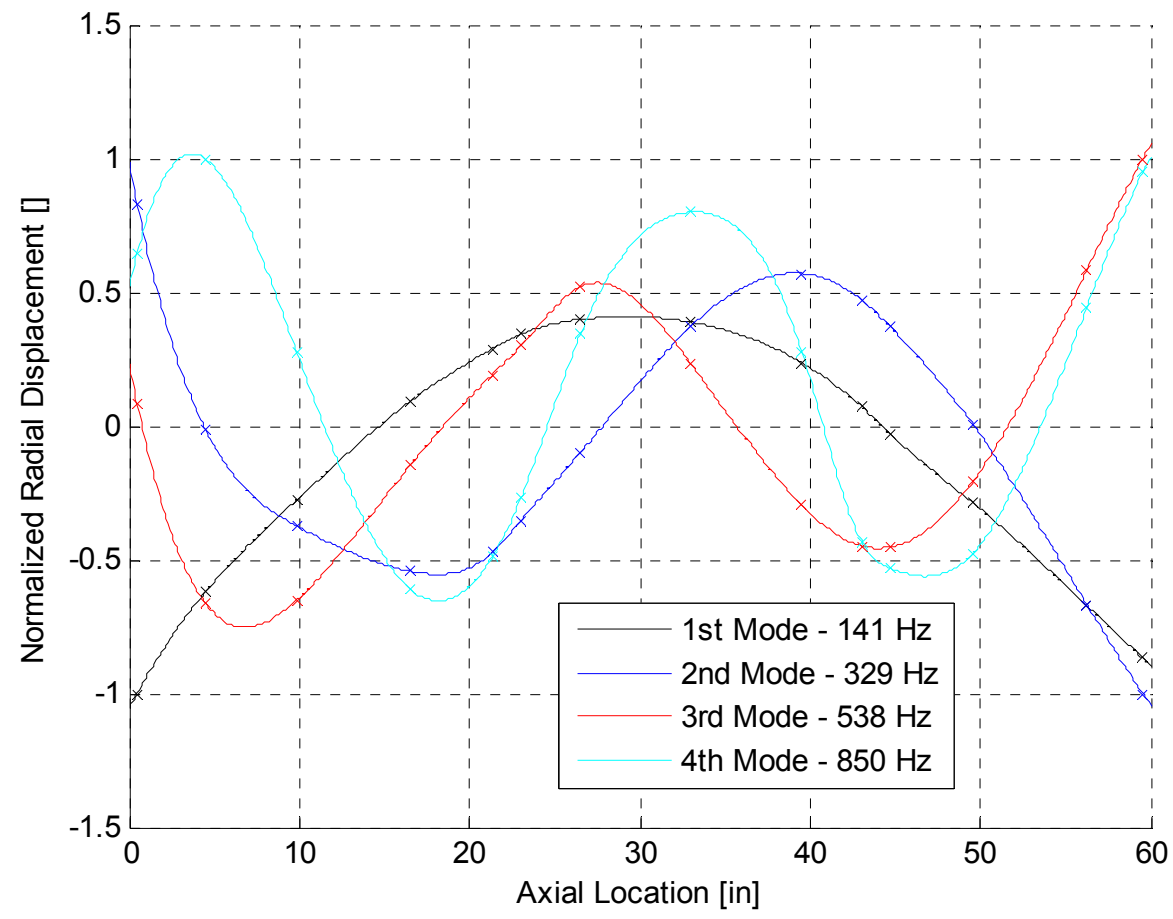

Fig. 44 Measured free-free rotor modes with coupling 

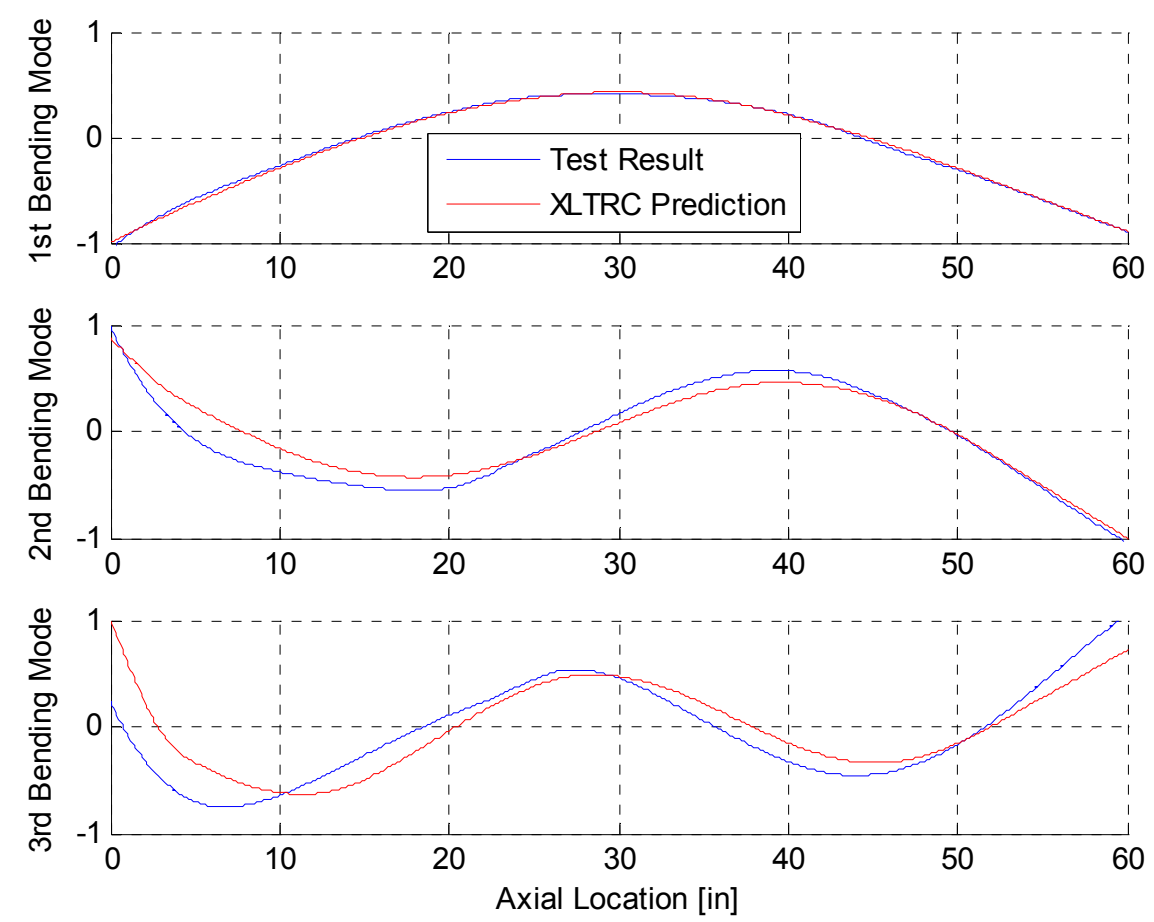

Fig. 45 Mode shape comparison between test result and $X L T R C^{2}$ prediction

The experimental modes, in particular the first and second, display a strong correlation with the FE predictions. The predicted natural frequencies of 140, 333, and $597 \mathrm{~Hz}$ also correlate with experimental results. The larger error associated with the third mode can be attributed to coupling model inaccuracies, as evidenced by the mode shape comparison at the drive end (left).

A free-free rap test was also performed without the coupling. Fig. 46 displays the mode shapes and frequencies. From the natural frequencies, the coupling produce a noticeables affect and is an essential component of an accurate FE system model. 


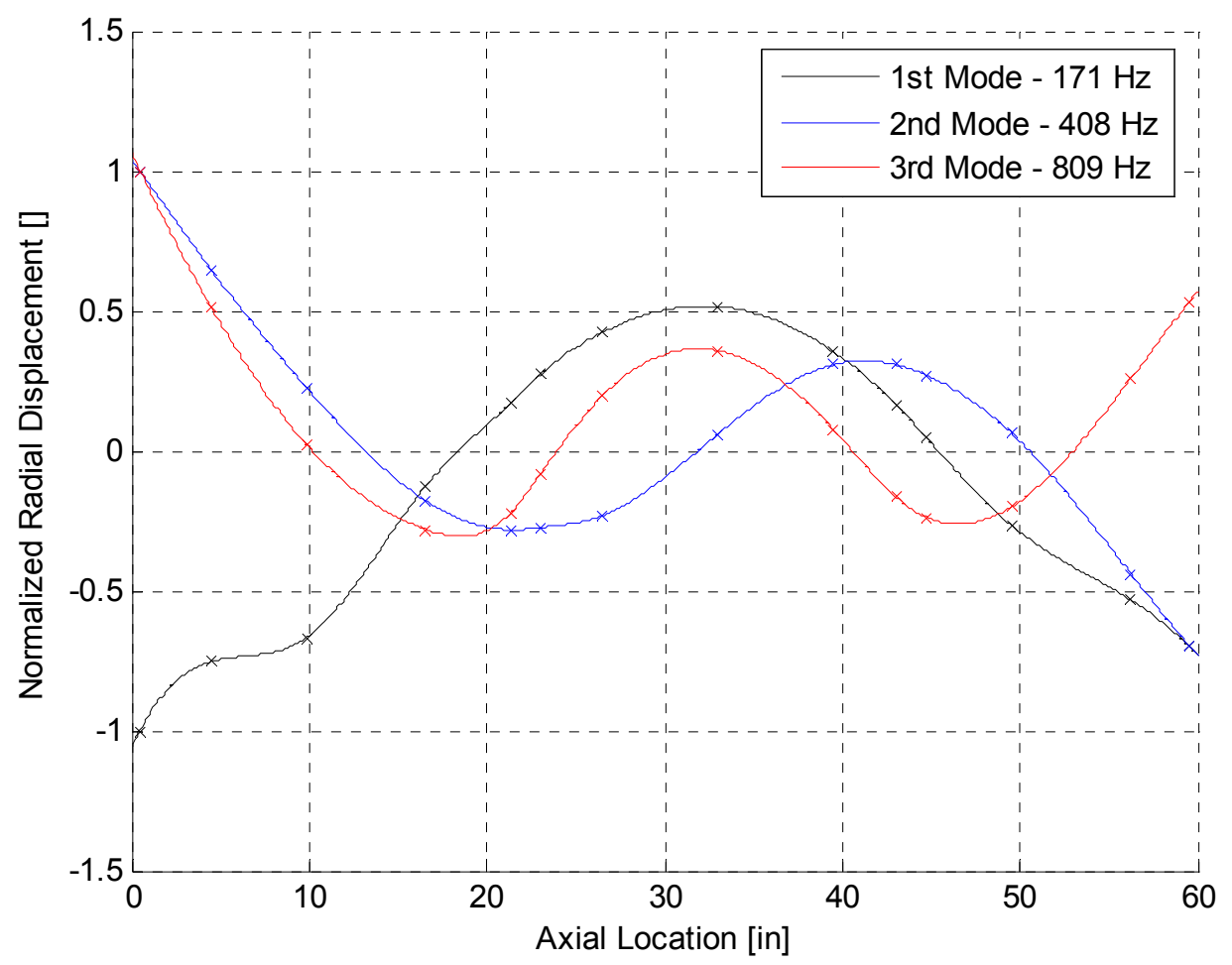

Fig. 46 Free-free rotor without coupling mode shapes

\section{Assembly Rap Test Results}

A rap test was also performed with the rotor-bearing system assembled and levitated. The rotor was excited in both the vertical and horizontal directions. The results are compared to determined effects of the MBs and support structure. XLTRC ${ }^{2}$ predictions are also computed for the two-bearing rotor model. These results are compared to determine the accuracy of the MB models.

Table 2 displays these results. The bending mode natural frequencies have excellent correlations for the horizontal and vertical excitations. This suggests that the influence of the support structure on these modes is either symmetric or negligible. Comparing the rigid mode natural frequencies from the vertical and horizontal tests demonstrates that 
the influence of the support structure is neither negligible nor symmetric. The conclusion is that the support structure flexibility is primarily caused by the rubber mat below the test stand. This mat is removed prior to the parameter identification addressed in later sections. At high frequencies, the large mass of the test stand isolates the rotorbearing system from the effects of the rubber mat. At low frequencies, the mat acts in series with the bearings and softens the system.

The softening effect can be observed by comparing the rigid modes of the experimental results with the XLTRC predictions. The bending mode predictions severely under predict the natural frequencies. This suggests that the magnetic bearing model (negative stiffness values and transfer functions) should be examined more closely if they are used in subsequent test programs.

Table 2 Rotor natural frequencies for assembled rap tests

\begin{tabular}{|c|c|c|c|}
\hline Mode & $\begin{array}{c}\text { Horizontal Excitation } \\
\text { Results (Hz) }\end{array}$ & $\begin{array}{c}\text { Vertical Excitation } \\
\text { Results (Hz) }\end{array}$ & $\begin{array}{c}\text { XLTRC Predictions } \\
(\mathbf{H z})\end{array}$ \\
\hline Cylindrical Mode & 23 & - & 50 \\
\hline Conical Mode & 41 & 35 & 77 \\
\hline $1^{\text {st }}$ Bending Mode & 164 & 161 & 142 \\
\hline $2^{\text {nd }}$ Bending Mode & 345 & 343 & 287 \\
\hline $3^{\text {rd }}$ Bending Mode & 551 & 551 & 365 \\
\hline $4^{\text {th }}$ Bending Mode & 873 & 873 & 627 \\
\hline
\end{tabular}

\section{Conclusions}

The results from the rap tests validate the rotor models used in predicting the DFTFs. They also demonstrate the influence of the coupling on the rotor-bearing system. The results from the assembled test demonstrate the influence of support structure flexibility, and illustrate the need for improved magnetic bearing models if these models are needed for future analysis. 


\section{FOSG Calibration}

The final aspect of testing to address is the FOSG calibration process. A variety of calibration methods are compared in [8]. The method found to be most effective involves exciting the rotor at frequencies well below the first bending mode, and correlating the strain measurements with the inertial force calculated using the rotor position measurements at the MBs. The approach uses a Matlab-based FE model to account for the effects of rotor flexibility. Accelerometers are also used to measure MB housing and test stand motion; these measurements are combined with the rotor position measurements to obtain the absolute position of the rotor.

The mathematical rigor behind this calibration has improved significantly during the course of this research project. This section will address the theory behind obtaining the FOSG calibration matrices. First, the rotor is excited at both MBs to produces a horizontal or vertical translation of the rotor. The rotor is excited with a $20 \mu \mathrm{m}$ amplitude at sequential frequencies of $3,15,25,35,45,55$, and $65 \mathrm{~Hz}$. Five seconds of steady state data are taken. The data are sampled at $10 \mathrm{kHz}$. The test is repeated 10 times at each frequency. The result is 70 horizontal excitations and 70 vertical excitations.

A FFT is performed on each set of data, and the amplitudes and phases at the test frequency are stored for each measurement. The amplitude and phase of the rotor absolute position are used to compute the inertial force generated by the rotor.

The strain measurements respond not only to a change in force applied to the rotor, but also a change in the rotor position $[8,18]$. The latter effect is explored later in the magnetic field FEA. At present, it is sufficient to relate the force applied at one $\mathrm{MB}\left(f_{x}\right.$ $\left.f_{y}\right)$ to the FOSG measurements $\left(s_{1}, s_{2}\right)$ and the rotor position $(x, y)$. Equation $(10)$ assigns the calibration matrices $\mathbf{C}_{\mathbf{S}}$ and $\mathbf{C}_{\mathbf{P}}$ to compute the force from the FOSG measurements and the rotor position. 


$$
\left[\begin{array}{l}
f_{x} \\
f_{y}
\end{array}\right]=\mathbf{C}_{\mathbf{S}}\left[\begin{array}{l}
s_{1} \\
s_{2}
\end{array}\right]+\mathbf{C}_{\mathbf{P}}\left[\begin{array}{l}
x \\
y
\end{array}\right]
$$

There are far more data points (140 tests with 6 known variables per MB) than necessary to compute an algebraic solution for the of the calibration matrices. A least-squares solution is desired. Equations (11) and (12) include vectors containing all force, strain, and position measurements from each test.

$$
\begin{gathered}
F_{x}=\left[\begin{array}{llll}
S_{1} & S_{2} & X & Y
\end{array}\right]\left[\begin{array}{l}
c_{S 11} \\
c_{S 12} \\
c_{P 11} \\
c_{P 12}
\end{array}\right]=\mathbf{H} C_{x} \\
F_{y}=\left[\begin{array}{llll}
S_{1} & S_{2} & X & Y
\end{array}\right]\left[\begin{array}{l}
c_{S 21} \\
c_{S 22} \\
c_{P 21} \\
c_{P 22}
\end{array}\right]=\mathbf{H} C_{y}
\end{gathered}
$$

The coefficients of the calibration matrices $\left(c_{S i j}\right.$ and $\left.c_{P i j}\right)$ can be estimated using a least squares solution.

$$
\begin{aligned}
C_{x} & =\left(\mathbf{H}^{T} \mathbf{H}\right)^{-1} \mathbf{H}^{T} F_{x} \\
C_{y} & =\left(\mathbf{H}^{T} \mathbf{H}\right)^{-1} \mathbf{H}^{T} F_{y}
\end{aligned}
$$

However, this solution is not an optimal solution because the measurements are not weight according to their uncertainties. The variances obtained from statistical analysis of the repeated tests can be used to weight the solution, resulting in a maximum- 
likelihood estimate. Additionally, the previous calculation does not allow weighting of the computed forces, which also vary during the repeated tests.

Gaussian least squares differential correction (GLSDC) is a nonlinear iterative approach to estimation. This method is now used to find optimal estimates for the calibration coefficients. The following computation is based on material from [5]. First, a measurement vector $\bar{Q}_{i}$ for each test $i$ is assembled.

$$
\bar{Q}_{i}=\left[\begin{array}{llllll}
f_{x} & f_{x} & s_{1} & s_{2} & x & y
\end{array}\right]_{i}^{T}
$$

The measurement vectors are combined into a vector $Q$ containing the measurements from all $n$ tests.

$$
Q=\left[\begin{array}{llll}
\bar{Q}_{1}^{T} & \bar{Q}_{2}{ }^{T} & \cdots & \bar{Q}_{n}{ }^{T}
\end{array}\right]^{T}
$$

Next, a vector of estimates for each test $i$ is assembled. The initial values are the actual strain and position measurements. This vector is updated with new estimates during each iteration.

$$
\bar{Z}_{i}=\left[\begin{array}{llll}
s_{1} & s_{2} & x & y
\end{array}\right]_{i}^{T}
$$

A full vector of the estimates including the calibration coefficient estimates is assembled.

$$
Z=\left[\begin{array}{llllll}
\bar{Z}_{1}^{T} & \bar{Z}_{2}^{T} & \cdots & \bar{Z}_{n}^{T} & C_{x}{ }^{T} & C_{y}{ }^{T}
\end{array}\right]^{T}
$$

A function $L$ computes the measurement estimates from the estimate vector $Z$ using its elements $z_{i}$. 


$$
L(Z)=\left[\begin{array}{c}
z_{1} \cdot z_{4 n+1}+z_{2} \cdot z_{4 n+2}+z_{3} \cdot z_{4 n+3}+z_{4} \cdot z_{4 n+4} \\
z_{1} \cdot z_{4 n+5}+z_{2} \cdot z_{4 n+6}+z_{3} \cdot z_{4 n+7}+z_{4} \cdot z_{4 n+8} \\
z_{1} \\
z_{2} \\
z_{3} \\
z_{4} \\
\vdots \\
z_{4 n-3} \cdot z_{4 n+1}+z_{4 n-2} \cdot z_{4 n+2}+z_{4 n-1} \cdot z_{4 n+3}+z_{4 n} \cdot z_{4 n+4} \\
z_{4 n-3} \cdot z_{4 n+5}+z_{4 n-2} \cdot z_{4 n+6}+z_{4 n-1} \cdot z_{4 n+7}+z_{4 n} \cdot z_{4 n+8} \\
z_{4 n-3} \\
z_{4 n-2} \\
z_{4 n-1} \\
z_{4 n}
\end{array}\right]
$$

The partial derivatives of the function $L$ with respect to the elements $z_{i}$ are computed for use in the GLSDC algorithm. The matrix $\overline{\mathbf{H}}$ is a submatrix of the matrix of partials $\mathbf{H}_{\mathbf{P}}$. The matrix of partials has the same number of rows as the measurement vector $Q$ and the same number of columns as the estimate vector $Z$ has rows

$$
\begin{gathered}
\overline{\mathbf{H}}=\left[\begin{array}{cccc}
z_{4 n+1} & z_{4 n+2} & z_{4 n+3} & z_{4 n+4} \\
z_{4 n+5} & z_{4 n+6} & z_{4 n+7} & z_{4 n+8} \\
1 & & & \\
& 1 & & \\
& & 1 & \\
& & & 1
\end{array}\right] \\
\mathbf{H}_{\mathbf{P}}=\left[\begin{array}{lll}
\overline{\mathbf{H}} & & \\
& \ddots & \\
& & \overline{\mathbf{H}}
\end{array}\right]
\end{gathered}
$$

The weight matrix $\mathbf{W}$ is composed of inverses of the variances obtained from statistical analysis of the repeated tests. The submatrix $\overline{\mathbf{W}}_{i}$ contains the variance terms associated 
with each tests. The weight matrix has the same number of rows and columns as the measurement vector $Q$ has rows.

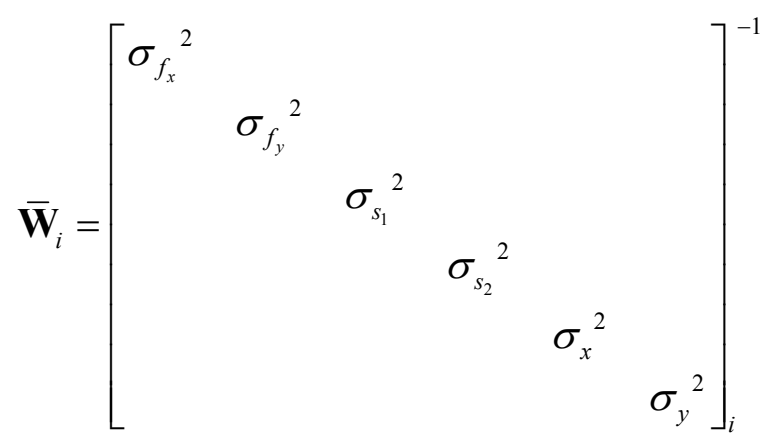

$$
\mathbf{W}=\left[\begin{array}{cccc}
\overline{\mathbf{W}}_{1} & & & \\
& \overline{\mathbf{W}}_{3} & & \\
& & \ddots & \\
& & & \overline{\mathbf{W}}_{n}
\end{array}\right]
$$

The GLSDC is implemented using the previously defined matrices. First the error between the measurements and the measurement estimates, the sum of the squares of the error, and the partials are computed.

$$
\begin{gathered}
\Delta Q=Q-L(Z) \\
J=\Delta Q^{T} \mathbf{W} \Delta Q \\
\mathbf{H}_{\mathbf{P}}=\mathbf{H}_{\mathbf{P}}(Z)
\end{gathered}
$$

Estimate corrections are computed using the weighted least squares method.

$$
\Delta Z=\left(\mathbf{H}_{\mathbf{P}}^{T} \mathbf{W H}_{\mathbf{P}}\right)^{-1} \mathbf{H}_{\mathbf{P}}{ }^{T} \mathbf{W} \Delta Q
$$

The estimate vector is updated and the process is repeated using the new estimate vector. 


$$
Z_{j+1}=Z_{j}+\Delta Z
$$

The algorithm terminates when the error sum $J$ falls below a specified value. The calibration coefficients are then available in the final estimate vector as they are assigned in Eq. (18). The calibration coefficients can then be used to compute force measurements for future tests from strain and positions measurements (Eq. (10)). With calibration coefficients, the forces can now be computed for a wide frequency range and for excitations that are more complicated than low frequency translations of the rotor.

One advantage of using the GLSDC solution is the ability to determine the estimation error covariance matrix from the measurement error covariance. The matrix $\mathbf{P}$ is the estimation error covariance matrix.

$$
\mathbf{P}=\left(\mathbf{H}_{\mathbf{P}}{ }^{T} \mathbf{W} \mathbf{H}_{\mathbf{P}}\right)^{-1}
$$

Typical values for the calibration matrices are listed in Table 3 with units of N/V (FOSG measurements are in Volts and are not converted to strain prior to force calculation). The table also includes the corresponding standard deviations from the estimation error covariance matrix. Note that calibration values vary between test dates noticeably more than indicated by the standard deviations $[8,18]$. The calibration values are determined immediately before each test to minimize the calibration drift.

\begin{tabular}{|c|c|c|c|c|c|c|c|c|}
\hline & \multirow{2}{*}{\multicolumn{2}{|c|}{$\frac{\mathrm{C}_{\mathrm{S}}{ }^{\mathrm{MB} 1}}{(\mathrm{~N} / \mathrm{V})}$}} & \multirow{2}{*}{\multicolumn{2}{|c|}{$\begin{array}{c}\mathrm{C}_{\mathrm{S}}{ }^{\mathrm{MB} 2} \\
(\mathrm{~N} / \mathrm{V})\end{array}$}} & \multirow{2}{*}{\multicolumn{2}{|c|}{$\frac{C_{\mathbf{P}}{ }^{\mathrm{MB1} 1}}{(\mathrm{~N} / \mu \mathrm{m})}$}} & \multirow{2}{*}{\multicolumn{2}{|c|}{$\frac{C_{\mathbf{P}}{ }^{\text {MB2 }}}{(\mathrm{N} / \mu \mathrm{m})}$}} \\
\hline & & & & & & & & \\
\hline \multirow{2}{*}{$\begin{array}{c}\text { Calibration } \\
\text { Matrices }\end{array}$} & -866.7 & 764.7 & -637.3 & 628.2 & -0.2769 & -0.5301 & 0.8525 & 0.7143 \\
\hline & 884.3 & 710.0 & 690.6 & 653.2 & -0.7907 & -1.2328 & -0.5598 & 0.8428 \\
\hline \multirow{2}{*}{$\begin{array}{c}\text { Standard } \\
\text { Deviations }\end{array}$} & 0.56 & 0.42 & 0.12 & 0.12 & 5.7E-05 & 5.0E-05 & $2.0 \mathrm{E}-05$ & 1.9E-05 \\
\hline & 0.57 & 0.43 & 0.13 & 0.13 & 5.9E-05 & 5.1E-05 & $2.2 \mathrm{E}-05$ & 2.1E-05 \\
\hline
\end{tabular}

Table 3 Typical FOSG calibration matrices and standard deviations 
Analyzing the calibration coefficients and standard deviations can yield the force measurement uncertainties. The force measurement uncertainty is the result of (i) the uncertainty in the FOSG measurement, and (ii) the uncertainty in the calibration coefficients. The FOSG SCU has an uncertainty of .025 micro-strain [20], which produces an SCU output voltage of $.012 \mathrm{~V}$. This results in an anticipated force measurement uncertainty of 11 to $13 \mathrm{~N}$ or .3 to $.4 \%$ of load capacity. The maximum SCU voltage amplitude for all tests is on the order of $1 \mathrm{~V}$; therefore, the uncertainty associated with the calibration coefficients is negligible as compared with the uncertainty due the FOSG measurement uncertainty. Additionally, the contributions of the position dependent strain $\left(\mathbf{C}_{\mathbf{P}}\right)$ are only slightly larger than the uncertainty for the test amplitude of $20 \mu \mathrm{m}$.

Previous results indicate that force measurement uncertainties of $5 \mathrm{~N}$ or $.1 \%$ of load capacity are achievable $[8,16,17,18,20]$. This is achieved by averaging test results or taking an FFT of tests containing many sinusoidal periods. The statistical effect is that the uncertainties are reduced. Taking an FFT of 1 second of FOSG data sample at 10 $\mathrm{kHz}$ reveals a maximum noise floor of .006 micro-strain for the test frequency range (Fig. 47). This supports previous findings that force measurement uncertainties of $5 \mathrm{~N}$ or .1\% of load capacity are achievable. 


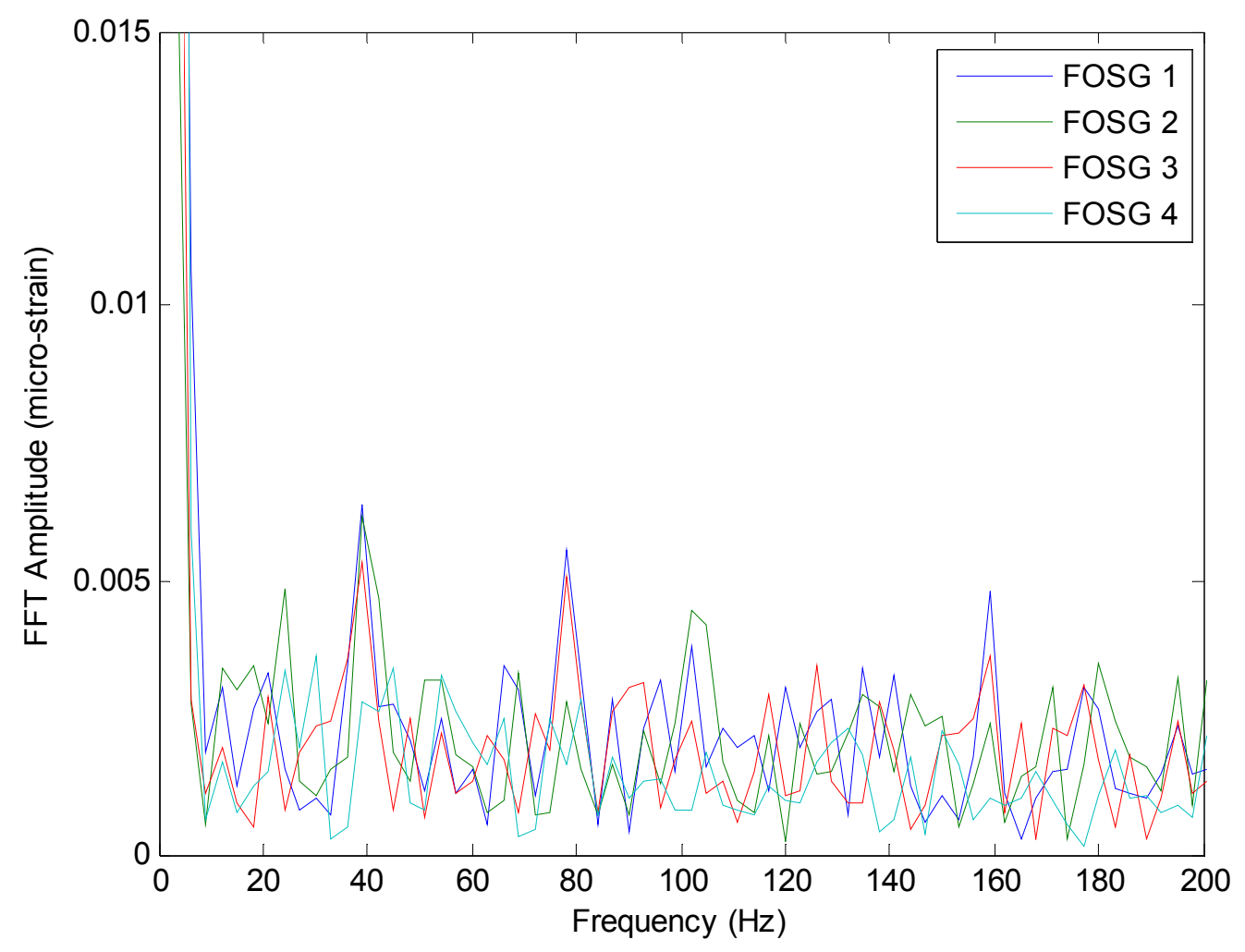

Fig. 47 FFT of FOSG noise 


\section{IDENTIFICATION OF SEAL COEFFICIENTS}

Parameter identification using magnetic bearings is the primary focus of this research project. More specifically, a parameter identification method applicable to real turbomachinery is demonstrated by estimating the relevant air seal coefficients at a variety of test conditions. The parameter identification attempts use time domain and frequency domain approaches detailed below. Static tests will also be performed to measure the seal stiffness coefficients

\section{Seal Coefficients}

Seals are used in turbomachinery to limit the leakage of fluid from a high pressure region to a low pressure region. Leakage negatively impacts the turbomachine efficiency and is therefore undesirable. Some seals such as brush seals make physical contact with the rotor and have very low leakage characteristics as a result. Brush seals have disadvantages including a pressure drop limitation across the seal.

Non-contacting annular seals limit leakage by allowing only a minimal clearance between the rotor and the seal. These seals also have a variety of mechanism to further reduce the leakage. Grooves or teeth can be added to the rotor and/or the seal to inhibit the fluid flow through the seal. Grooved seals and labyrinth seals can have a negative impact on turbomachine stability [21]. Honeycomb and hole pattern seals can produce similar leakage characteristic without reducing the turbomachine stability [22,23]. Specialized seals such as pocket damper seals can actually improve stability characteristics by producing damping forces [24]. Perhaps the most basic type of noncontacting seal is the smooth seal [25]. For this seal, the clearance, diameter, and length are the only geometric properties affecting the fluid leakage. These seal types are displayed in Fig. 48. 


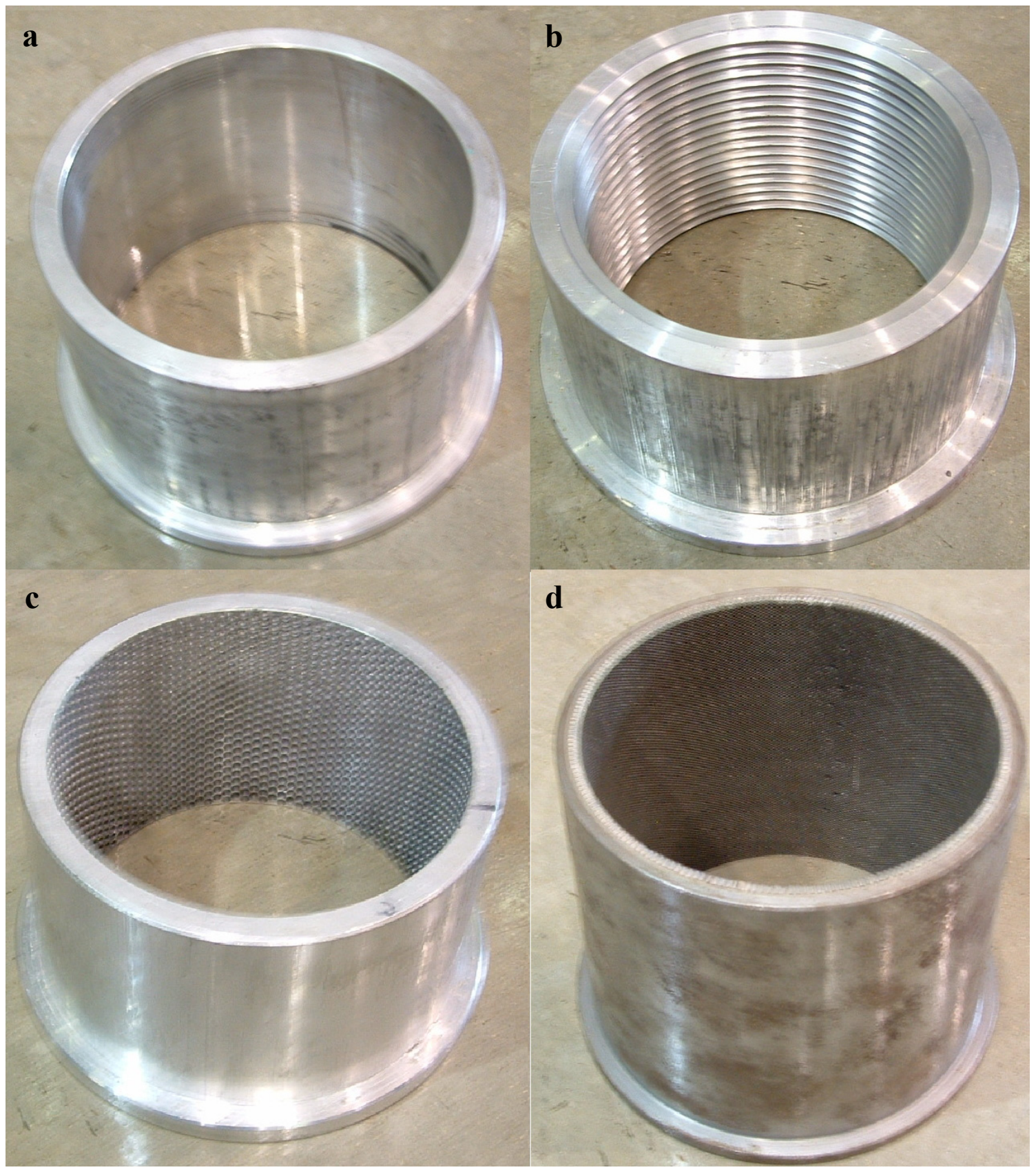

Fig. 48 Seal pictures- (a) smooth, (b) labyrinth, (c) hole pattern, (d) honeycomb

Historical and current test results for smooth air seals indicate that the model from Eq. (30) is representative of smooth seal forces for an eccentricity ratio of less than 0.5 [25]. The radial forces $f_{x}$ and $f_{y}$ applied to the rotor are modeled as stiffness and damping 
forces associated with radial displacements and velocities or the rotor at the seal. Note that the diagonal terms are equal, and the off diagonal terms are equal and opposite. In addition, the cross-coupled damping forces are considerably smaller than the other forces and are neglected for the purposes of identification [26]. This limits the number of parameters for identification to 3 . The focus of the present research is on identifying these parameters. The hole pattern and honeycomb seals mentioned previously have frequency dependent force coefficients, and they would require a more complex model than the air seal model.

$$
\left[\begin{array}{l}
f_{x} \\
f_{y}
\end{array}\right]=-\left[\begin{array}{cc}
C & c \\
-c & C
\end{array}\right]\left[\begin{array}{l}
\dot{x} \\
\dot{y}
\end{array}\right]-\left[\begin{array}{cc}
K & k \\
-k & K
\end{array}\right]\left[\begin{array}{l}
x \\
y
\end{array}\right]
$$

The swirl rings, shown in the air seal assembly description (Fig. 7) are used to simulate conditions of a real turbomachine. The air entering a seal in a real turbomachine may already have a significant circumferential velocity. This characteristic contributes substantially to the cross-coupled stiffness $k$; specifically, increasing the inlet tangential velocity increases $k$. Theoretical calculations for the seal coefficients predict a significant impact on the rotor response characteristics due to the air seal. This suggests a high probability of successfully identifying the seal coefficients.

\section{Test Conditions}

Table 4 contains the identification test matrix. The test variables are the rotor speed and the air seal preswirl. As mentioned previously, time and frequency domain identification approaches are attempted. The frequency domain identification produces excellent result that are detailed later in this section. The time domain attempt did not produce valid identification results. The methods attempted and the problems that were encountered are discussed in the section on time domain identification. The air seals are tested with no preswirl and with high preswirl. The high preswirl condition is determined by the geometry of the preswirl ring pressed into the air seal. Test results are 
obtained at 0 speed and the maximum test speed of $7700 \mathrm{rpm}$. 10 sets of data are collected for the $0 \mathrm{rpm}$ frequency domain cases. 1 set of data is collected for the other cases. A set of data consists of sufficient experimental measurements in the frequency range of the first bending mode $(140 \mathrm{~Hz})$ for identification. The 10 data sets from the 0 rpm frequency domain cases are used to calculate uncertainties for the frequency domain identification. In addition to the test mentioned previously, a second test on a different test date is performed for each condition, to establish the repeatability of the identification methods.

Static measurements of the stiffness coefficients are also performed at three operating conditions: non-rotating with and without preswirl, and rotating with preswirl. The measurements use an empirical MB model based on the MB control currents and rotor position to calculate forces without using FOSGs. These measurements are compared with results from the previously mentioned identification procedures.

Table 4 Identification test matrix

\begin{tabular}{|r|c|c|c|c|}
\hline \multicolumn{4}{|c|}{ Number of Data Sets for Various Test Conditions } \\
\hline $\begin{array}{r}\text { Speed } \\
\text { (rpm) }\end{array}$ & Frequency Domain ID & Static Measurements \\
\hline $\mathbf{0}$ & No Preswirl & High Preswirl & No Preswirl & High Preswirl \\
\hline $\mathbf{8 0 0 0}$ & 1 Sets & 10 Sets & 1 Meas. & 1 Meas. \\
\hline
\end{tabular}

The frequency range is centered about the first bending mode natural frequency because the system response exhibits a dramatic change due to the seal coefficients at these frequencies. To predict the change, dynamic flexibility transfer functions (DFTFs) are computed with an FE model that is detailed in the following section. Equation (31) displays the function of the DFTF matrix $\mathbf{G}$ as it relates to the force vector $F$ and position vector $X$. Each element of the DFTF matrix in Eq. (31) is a complex valued function that varies with excitation frequency to represent the amplitude and phase of the system response $X$ to force $F$. A detailed description of DFTFs and computation 
methods is found in the previous section. Both forces and positions are measured in the horizontal and vertical directions at each bearing (denoted by $M B 1$ and $M B 2$ ).

$$
\begin{aligned}
X & =\mathbf{G} F \\
X & =\left[\begin{array}{l}
x_{M B 1} \\
y_{M B 1} \\
x_{M B 2} \\
y_{M B 2}
\end{array}\right] \\
\mathbf{G} & =\left[\begin{array}{llll}
G_{11} & G_{12} & G_{13} & G_{14} \\
G_{21} & G_{22} & G_{23} & G_{24} \\
G_{31} & G_{32} & G_{33} & G_{34} \\
G_{41} & G_{42} & G_{43} & G_{44}
\end{array}\right] \\
F & =\left[\begin{array}{l}
f_{x}^{M B 1} \\
f_{y}^{M B 1} \\
f_{x}^{M B 2} \\
f_{y}^{M B 2}
\end{array}\right]
\end{aligned}
$$

Fig. 49 shows the predicted DFTFs with and without the air seal. The response with the seal is calculated using seal coefficient predictions from the air seal design analysis. The graphs plot the amplitudes of the DFTFs vs. frequency with chart position indicating the respective position within the DFTF matrix (Eq. (31)). The direct damping term dramatically decreases the peak amplitudes in the direct DFTFs, while the cross-coupled stiffness creates a cross-coupled response. The direct stiffness has a slight effect on the location of the natural frequency. The effect of the coefficients was determined by adding each coefficient to the model separately and observing the resulting changes. The modeled DFTFs indicate that the frequencies about the first bending mode natural frequency are a reasonable selection for identification of the seal parameters. 

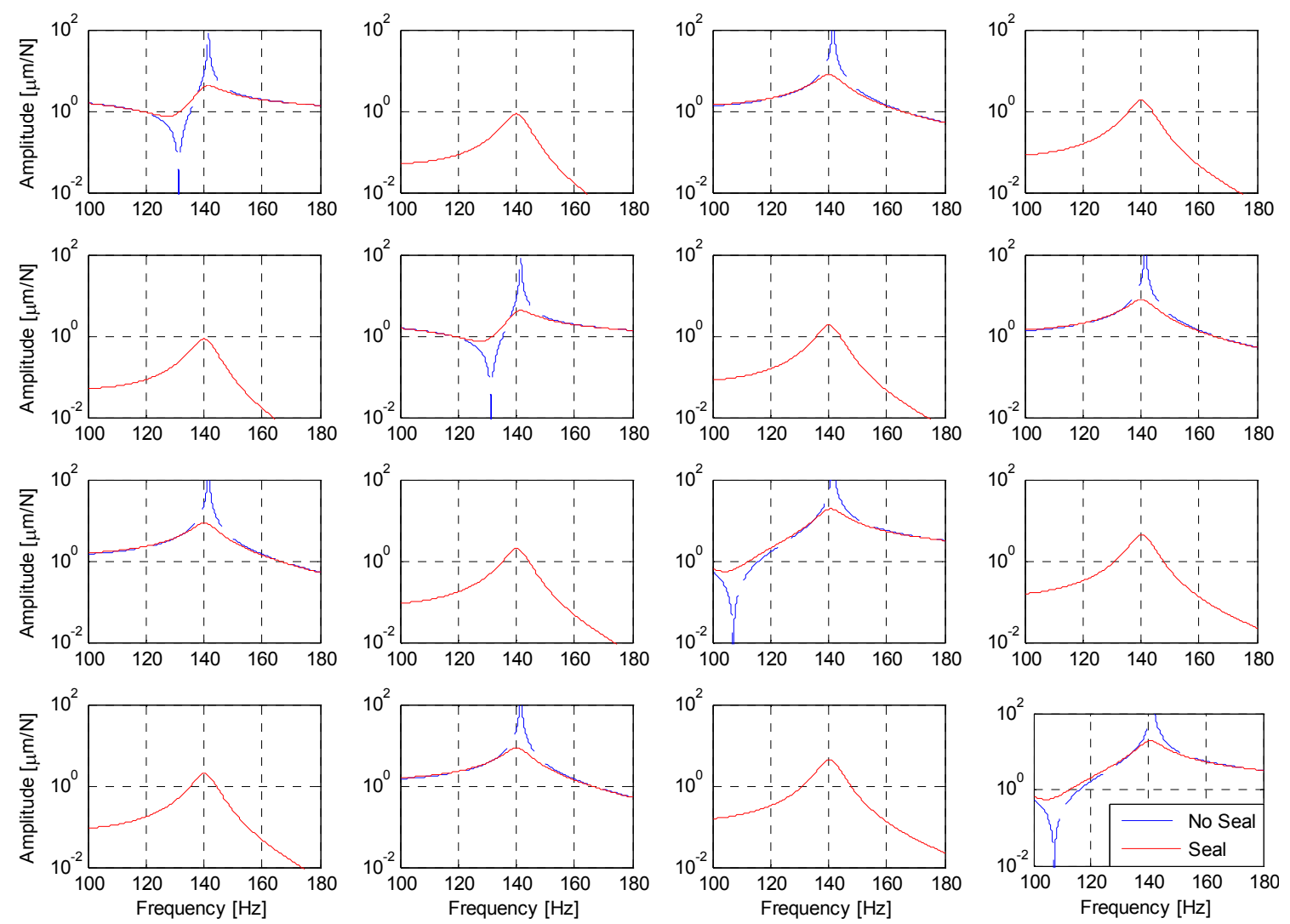

Fig. $49 G_{i j}$ (Eq. (31)) FE model DFTF predictions with and without seal

\section{FE Rotor Model}

Both identification methods described later require an accurate model of the rotor. The rotor model used in the previous analysis was developed using the Turbomachinery Laboratory rotordynamics program $X L T R C^{2}$. A new model was developed using in Matlab to facilitate the identification process. The Matlab FE model is also used in the FOSG calibration procedure to account for rotor flexibility. 
The rotor is modeled using finite elements described in [3]. At constant running speed $\omega$, Eq. (32) displays the general linear equation of motion for a free-free rotor. The matrices are developed from the rotor geometry and mechanical properties [27].

$$
\mathbf{M} \ddot{q}-\omega \mathbf{G} \dot{q}+\mathbf{K} q=F
$$

The vector of generalized coordinates $q$ includes 4 degrees-of-freedom at each station along the length of the rotor. Radial displacements of the rotor along two orthogonal axes are included as are rotations about those axes. Equation (33) shows the arrangement of the displacement and rotation variables within the generalized coordinate vector.

$$
q=\left[\begin{array}{lllllllll}
x_{1} & y_{1} & \beta_{x_{1}} & \beta_{y_{1}} & \cdots & x_{n} & y_{n} & \beta_{x_{n}} & \beta_{y_{n}}
\end{array}\right]^{T}
$$

This model includes a reduction for the disc and rotor laminate stiffness, as in the $X L T R C^{2}$. However, this model is improved by further refinement of the disc stiffness value and the coupling inertial properties. The model is tuned to match the natural frequencies and mode shapes for the first three free-free bending modes. The resulting good degree of model-experimental agreement ensures that the model accurately captures necessary dynamics of the rotor. The process of matching frequencies and mode shapes is detailed in the following section.

The seal coefficients contained in the seal damping and stiffness matrices, $C_{s}$ and $K_{s}$ respectively, can be readily added to the rotor model. For the parameter identification process, the rotor model matrices are known (constants), and the seal coefficient matrices are treated as unknown (variables). 


$$
\begin{aligned}
& F_{s}=-C_{s} \dot{q}-K_{s} q \\
& M \ddot{q}+\left(C_{s}-\omega G\right) \dot{q}+\left(K_{s}+K\right) q=F \\
& C_{s}=\left[\begin{array}{lllll}
0 & & & & \\
& \ddots & & & \\
& & C & 0 & \\
& & 0 & C & \\
& & & & \ddots
\end{array}\right] \\
& K_{s}=\left[\begin{array}{lllll}
0 & & & & \\
& \ddots & & & \\
& & K & k & \\
& & & K & \\
& & & &
\end{array}\right]
\end{aligned}
$$

\section{Frequency Domain Identification}

The frequency domain identification is accomplished by experimentally measuring DFTFs using the MBs, and then matching the experimental results with modeled DFTFs. Modeled DFTFs from the FE rotor-seal model (Eq. (34)) are fitted to the experimental DFTFs using a standard non-derivative search minimization algorithm. The seal parameters are identified as coefficients that minimize the theoretical vs. experimental error in the least-squares sense. Similar frequency domain identification approaches are outlined in the literature by Maslen and Wang et al. [28,29,30]

DFTFs are measured experimentally by taking an FFT of the rotor position and applied force at the MBs. The excitation frequencies are tightly space about the first bending mode natural frequency from 120 to $160 \mathrm{~Hz}$. The DFTF amplitudes and phases at each frequency characterize the matrix G. Experimental DFTF computation is addressed in the previous section. For the $0 \mathrm{rpm}$ cases, the tests are repeated 10 times to obtain uncertainties at each frequency. Using these results an uncertainty model based on DFTF amplitude is developed. The theoretical DFTFs are computed by obtaining the 
steady state system response to unit amplitude force excitation at each input for each test frequency. The process of determining the seal coefficients is detailed below.

1. The experimental DFTFs and uncertainties are measured. Experimental DFTF computation is addressed in the previous section. DFTF uncertainties are characterized by calculating the standard deviations in the test results for repeated tests ate $0 \mathrm{rpm}$.

2. The FE DFTFs are computed using an initial set of seal coefficient values (predictions from a seal code) for the seal coefficients. The rotor FE model (excluding seal terms) is known and is not altered during the identification process.

3. The errors between the measured DFTFs and FE DFTFs are computed at each frequency $j$. The total error vector $e$ is a column vector of all the errors for each transfer function and each frequency.

$$
\begin{aligned}
\mathbf{E}_{\mathbf{j}} & =\mathbf{G}_{\mathbf{j}}^{\text {measured }}-\mathbf{G}_{\mathbf{j}}^{\text {predicted }} \\
\mathbf{E}_{\mathbf{j}} & =\left[\begin{array}{llll}
E_{j, 1} & E_{j, 2} & E_{j, 3} & E_{j, 4}
\end{array}\right] \\
e_{j} & =\left[\begin{array}{c}
E_{j, 1} \\
E_{j, 2} \\
E_{j, 3} \\
E_{j, 4}
\end{array}\right] \\
e & =\left[\begin{array}{c}
e_{1} \\
e_{2} \\
\vdots \\
e_{n}
\end{array}\right]
\end{aligned}
$$

4. The sum of the error squares $J$ is computed. The error terms are initially weighted inversely to the corresponding variances to obtain a maximum likelihood solution. However, test rig factors (addressed in the frequency domain conclusions) cause an unweighted error calculation to produce superior results. 


$$
J=e^{T} e
$$

5. A multidimensional unconstrained nonlinear minimization algorithm know as the Nelder-Mead simplex method is used to iterate (repeating steps 2 through 4) with new FE model seal coefficients to find the local minimum of $J$ [31].

6. Once the seal coefficients are identified, a Monte Carlo type uncertainty analysis is performed. The experimental DFTF values are perturbed with errors from the uncertainty model. The errors are normally distributed with standard deviations based on the repeated test results. The identification process is repeated with for 10 sets of perturbed experimental DFTFs. A statistical analysis of the seal coefficient identification results yields standard deviations for each of the identified seal coefficients, reflecting the uncertainty in the identification of each coefficient.

The parameter values that minimize $J$ solve the local least squares minimization problem. If convergence is achieved, the parameters are therefore guaranteed (locally) to achieve the best fit in the least squares sense.

\section{Frequency Domain DFTF Comparison}

The frequency domain identification results indicate a remarkably accurate identification. Prior to displaying identification results, displaying a comparison of the measured DFTFs without the seal to the DFTFs using the new FE model is helpful. Fig. 50 and Fig. 51 display the amplitudes and phases, respectively, of the experimental and modeled DFTFs with the air seal. The subplot locations reflect the position within the DFTF matrix from Eq. (31). Some of the plots are omitted because there is no predicted response for those DFTF terms. The excellent agreement of both the amplitude and phase properties is a strong indication of an accurate system model. 

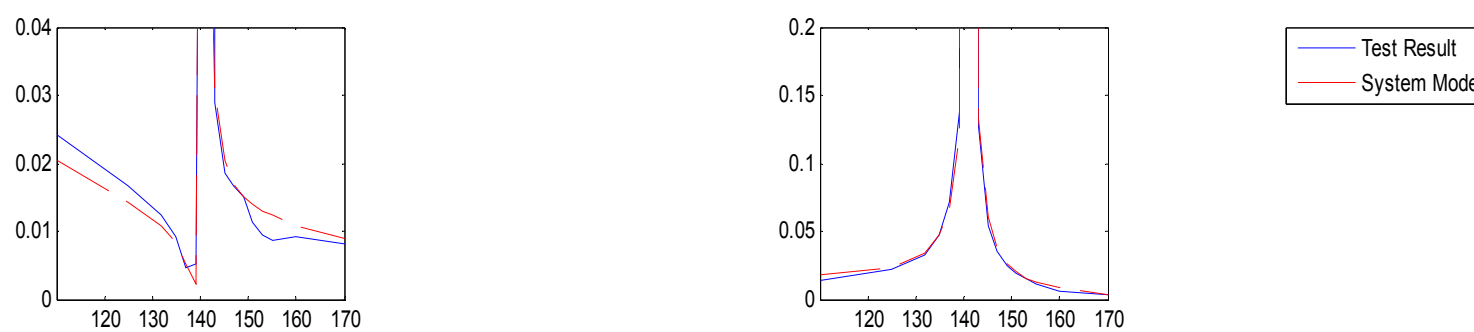

System Model
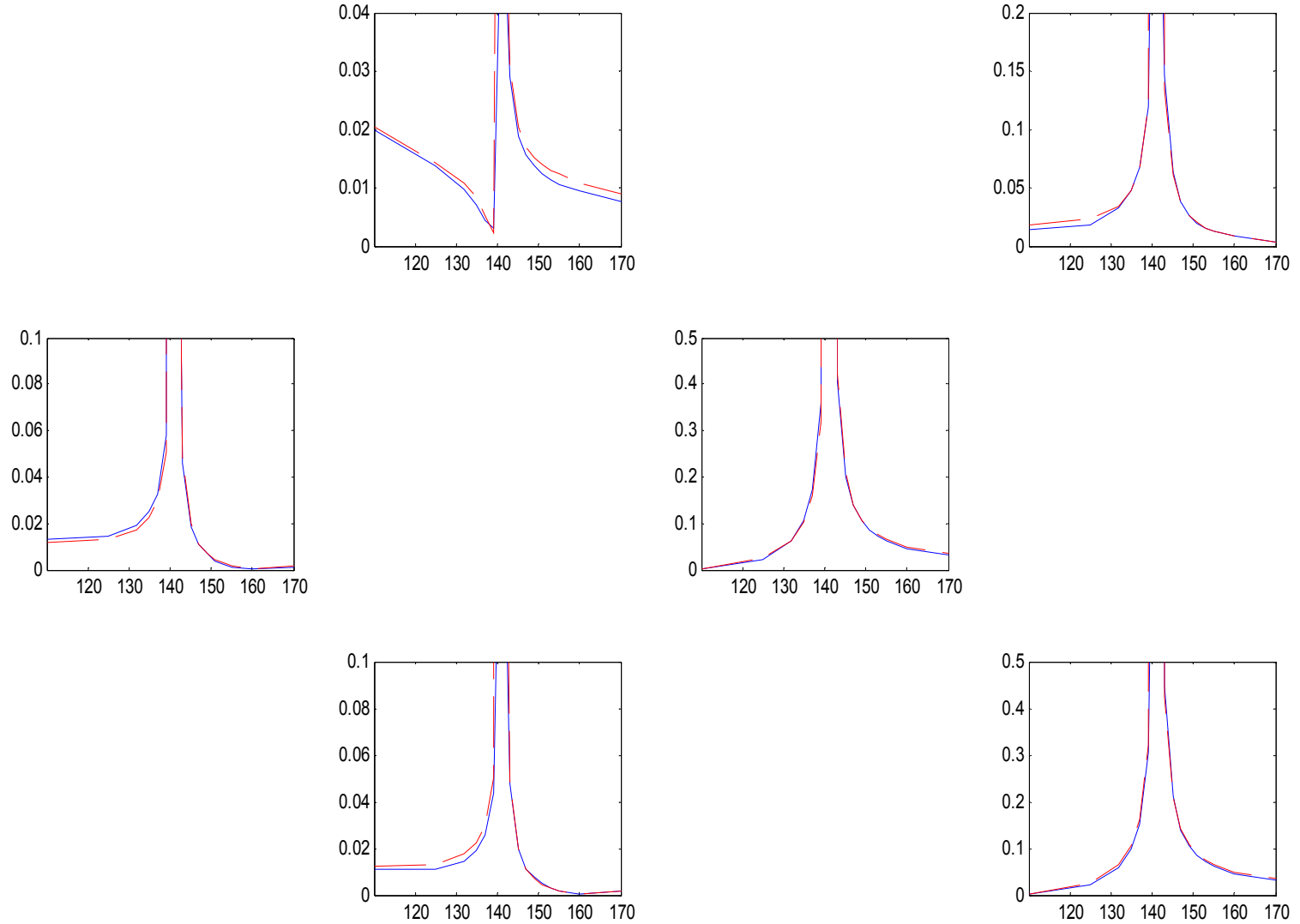

Frequency [Hz]

Fig. 50 Comparison of experimental and modeled DFTF amplitudes $(\mu \mathrm{m} / \mathrm{N})$ without air seal 

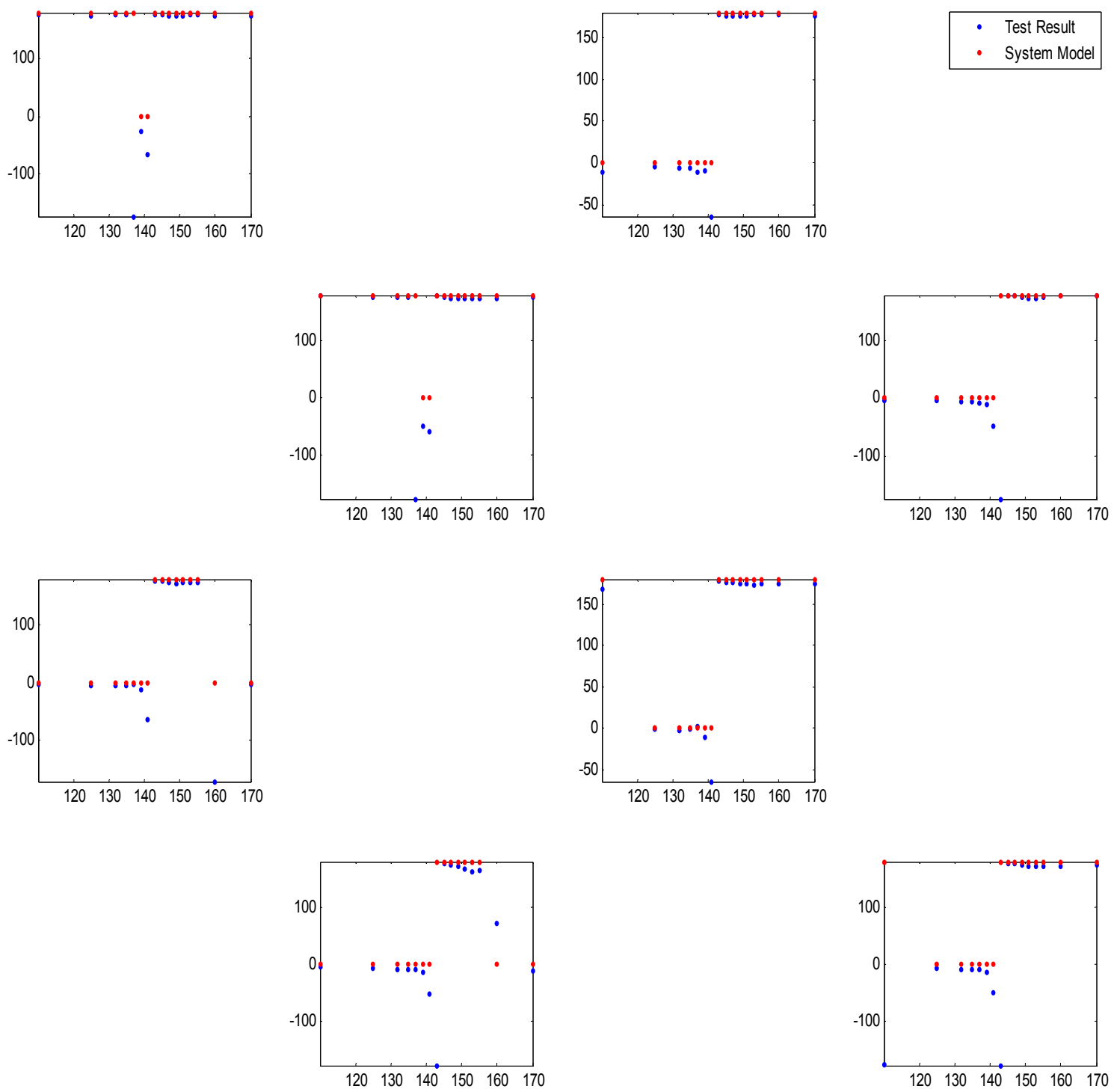

Frequency $[\mathrm{Hz}]$

Fig. 51 Comparison of experimental and modeled DFTF phases $\left({ }^{\circ}\right)$ without air seal

The results for the first test case, $0 \mathrm{rpm}$ without preswirl, are shown in Fig. 52 and Fig. 53. Strong agreement is, again, an indication of the accuracy of the system model. Uncertainty levels (standard deviations) are plotted on the amplitude plots. True measured uncertainties and modeled uncertainties are displayed for comparison. There is a reasonable agreement between measured and modeled uncertainties. The identified 
coefficients for all cases are tabulated later in this section. The results display noticeable damping and a slight reduction in natural frequency. The coupling measured between the horizontal and vertical directions is minimal.
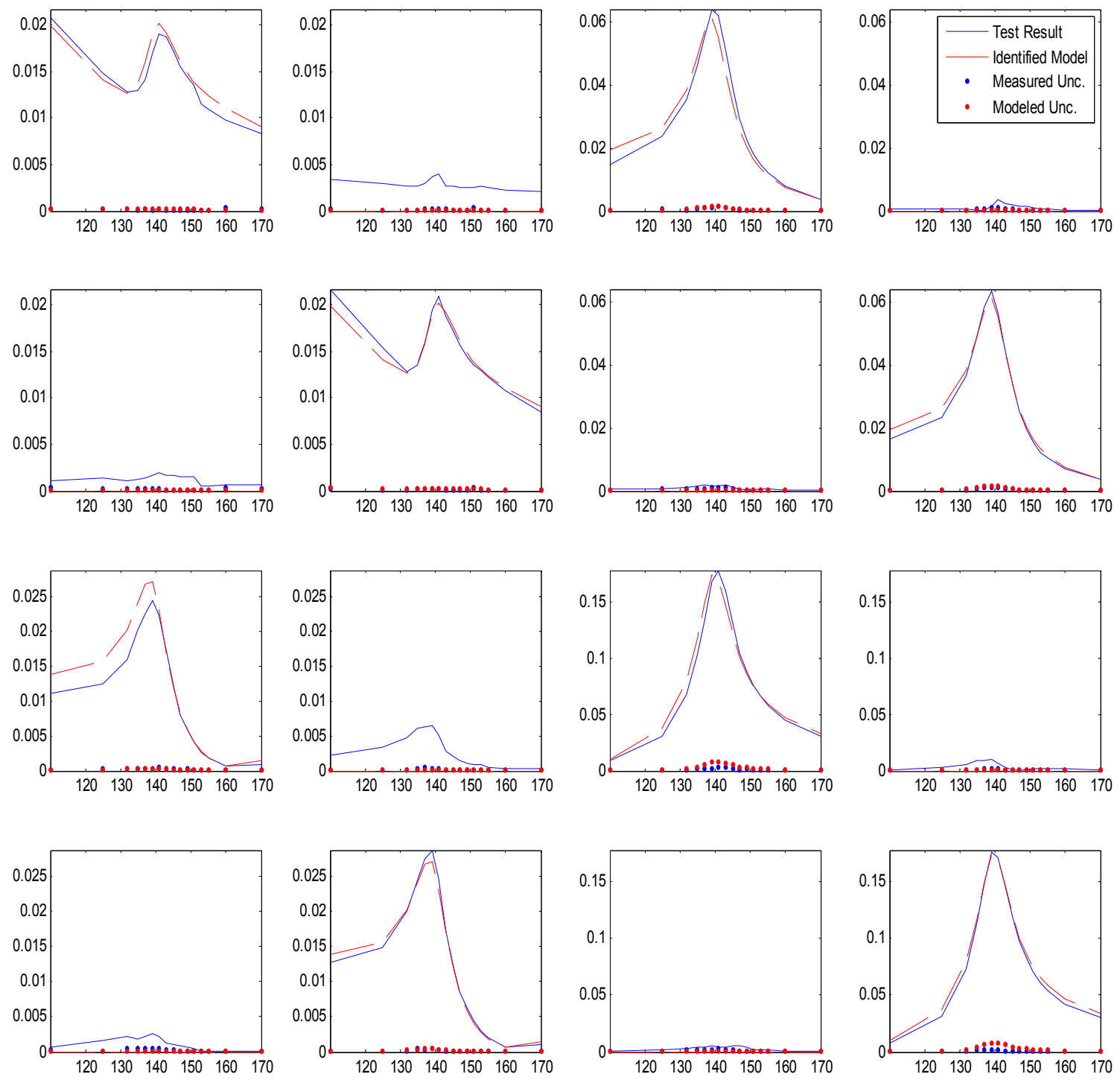

Frequency [Hz]

Fig. 52 Frequency domain identification amplitudes $(\mu \mathrm{m} / \mathrm{N})$ at $0 \mathrm{rpm}$ without preswirl 

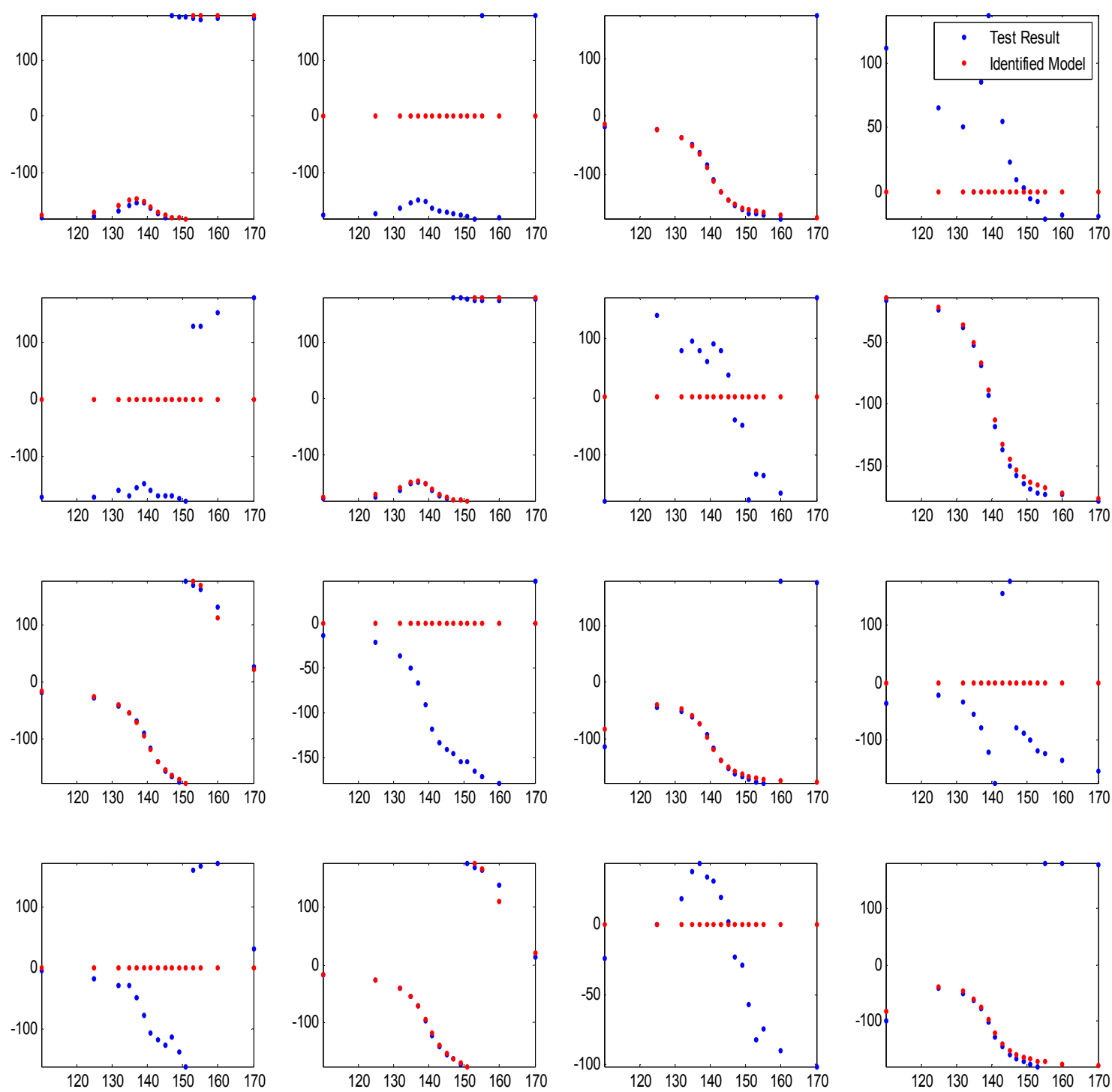

Frequency $[\mathrm{Hz}]$

Fig. 53 Frequency domain identification phases $\left({ }^{\circ}\right)$ at $0 \mathrm{rpm}$ without preswirl

Fig. 54 and Fig. 55 display the identification results for the second case, 7700 rpm without preswirl. The experimental and modeled DFTFs, and the modeled uncertainties are shown. This case exhibits the most significant discrepancies of any cases. This is presumably because the coupling between the horizontal and vertical directions, while noticeable is small as compared with results from the cases with preswirl. The cross- 
coupled stiffness for this case is difficult to detect with the strong gyroscopic influence on the test results. Clearly the uncertainties based on repeated tests are noticeably smaller than the experimental-theoretical error. This suggests that there are sources of uncertainty in the test procedure that are not adequately captured by repeating test over a short time frame. For this reason, all tests are repeated at different test dates to verify repeatability of the seal coefficient identification.
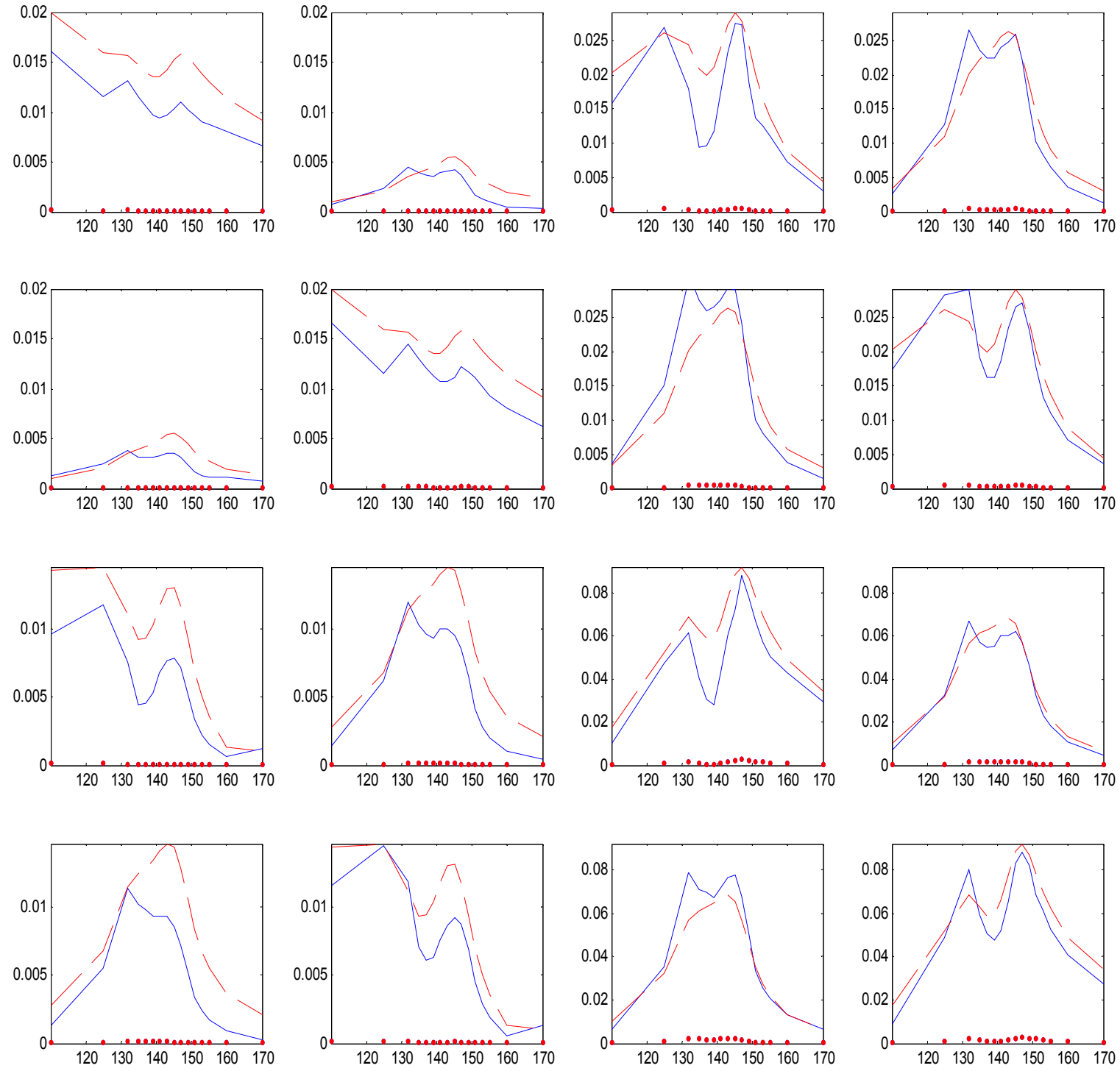

Frequency $[\mathrm{Hz}]$

Fig. 54 Frequency domain identification amplitudes $(\mu \mathrm{m} / \mathrm{N})$ at $7700 \mathrm{rpm}$ without preswirl 

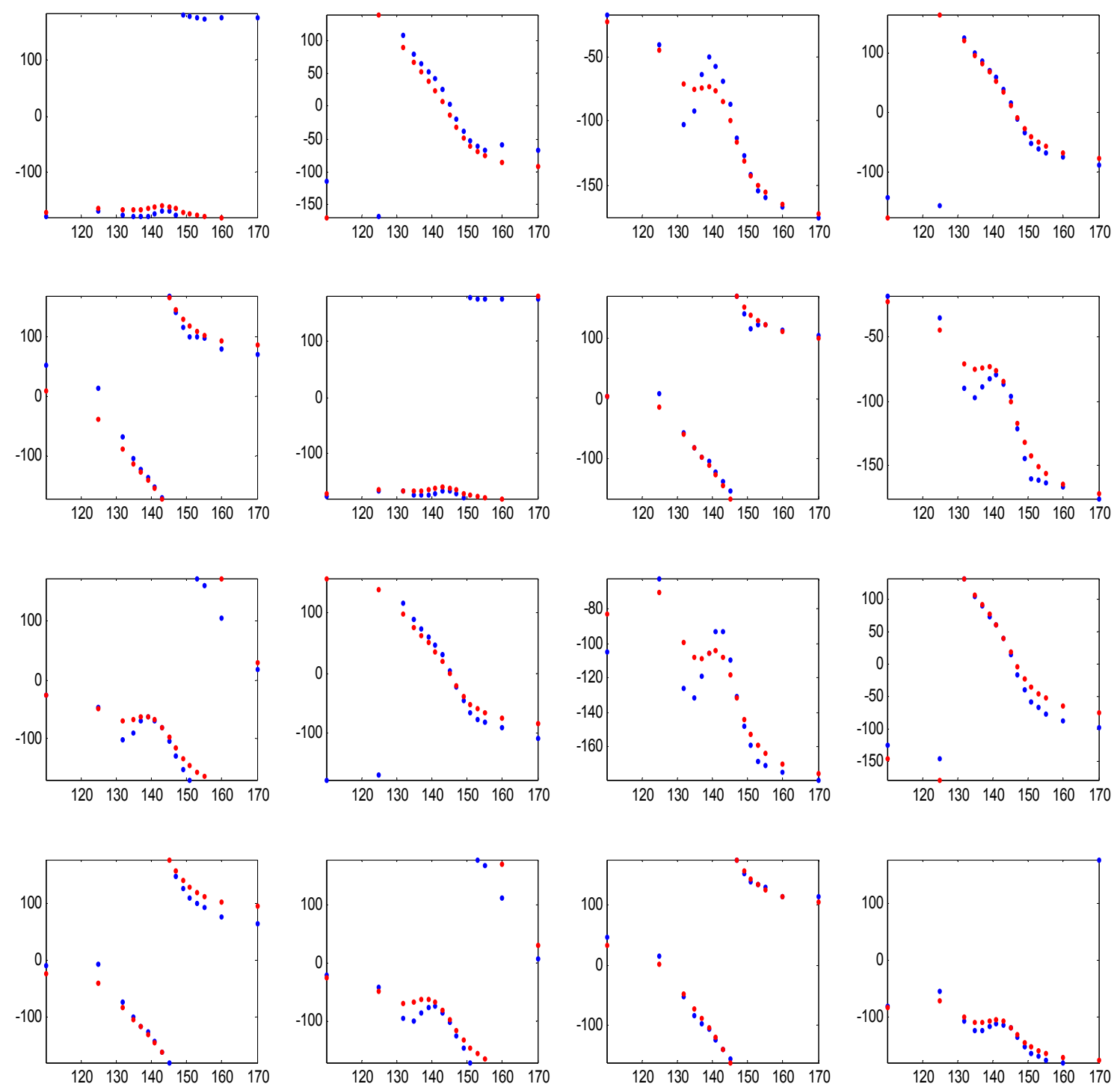

Frequency $[\mathrm{Hz}]$

Fig. 55 Frequency domain identification phases $\left(^{\circ}\right)$ at $7700 \mathrm{rpm}$ without preswirl

Fig. 56 and Fig. 57 display the results for the third test case, $0 \mathrm{rpm}$ with preswirl. The coupling between the vertical and horizontal directions is substantial. This is the result of the large cross-coupled stiffness caused by the preswirl. 

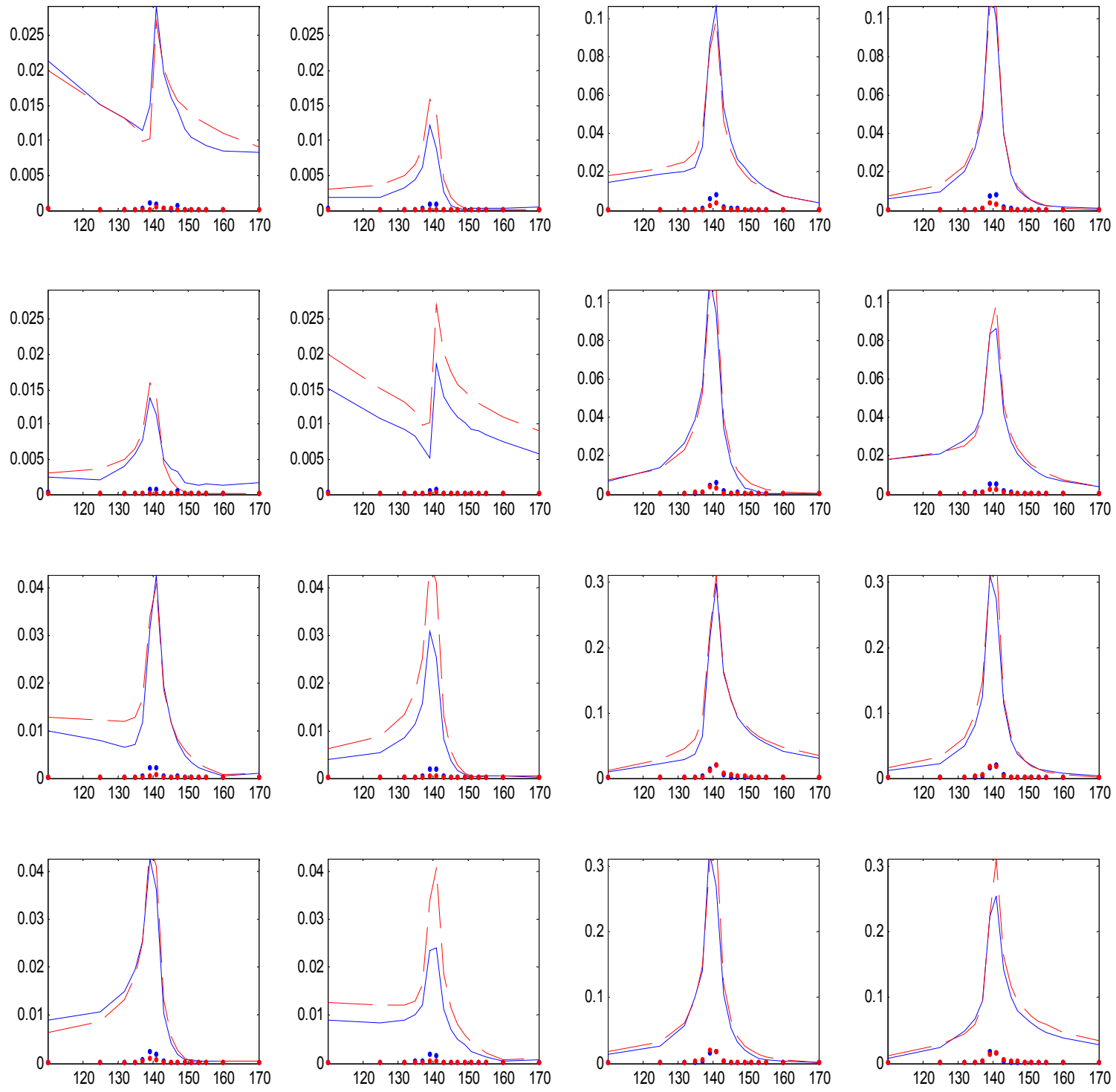

Frequency $[\mathrm{Hz}]$

Fig. 56 Frequency domain identification amplitudes $(\mu \mathrm{m} / \mathrm{N})$ at $0 \mathrm{rpm}$ with preswirl 

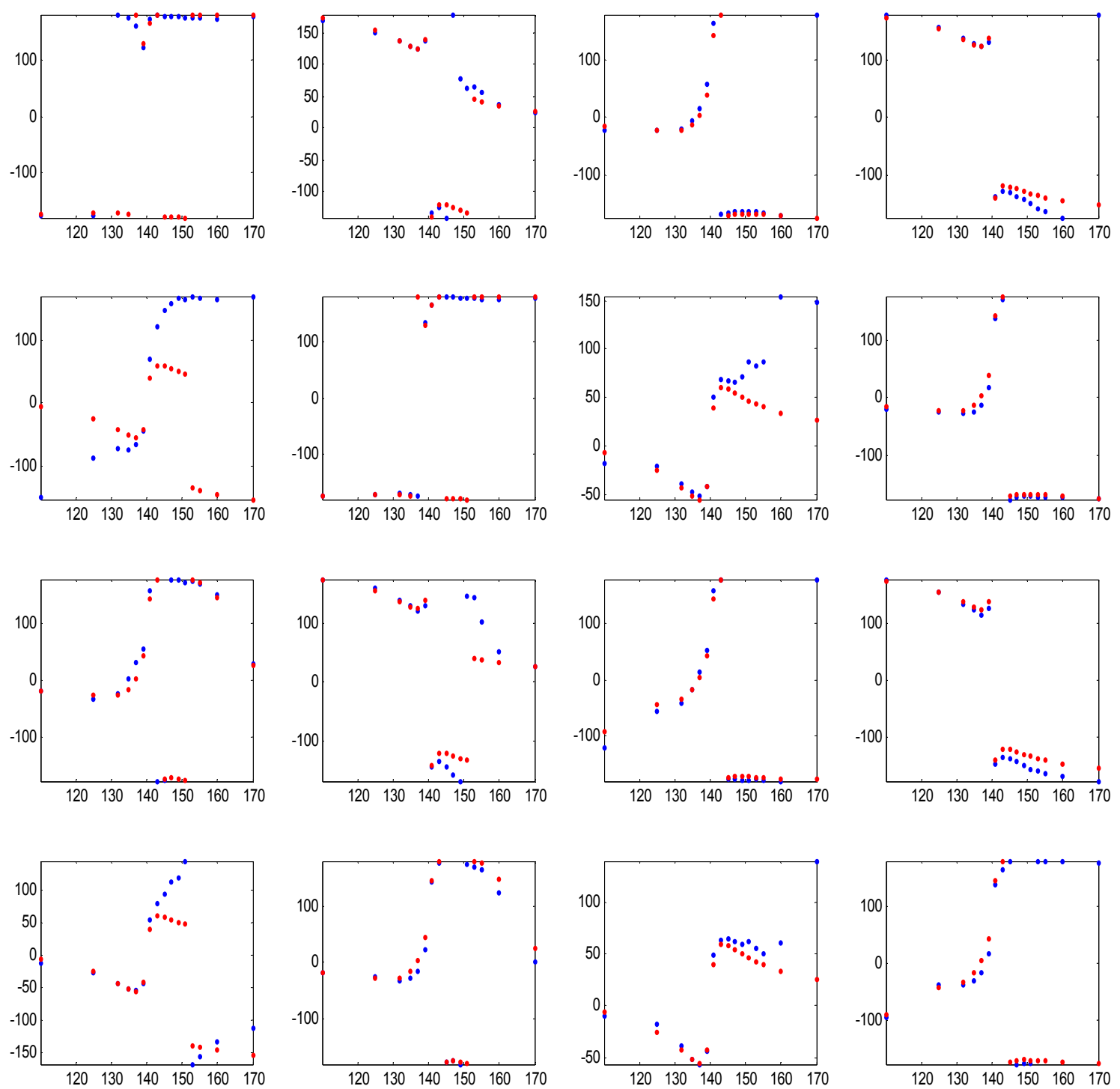

Frequency $[\mathrm{Hz}]$

Fig. 57 Frequency domain identification phases $\left({ }^{\circ}\right)$ at 0 rpm with preswirl

Initially, testing the preswirl configuration is not possible because the full rotor-bearing system becomes unstable when the pressurized air was added to the seal. Fig. 58 displays the unstable transient response of the system. Subscripts $D$ and $N D$ indicate the drive and non-drive MBs respectively. The instability occurs as the supply air pressure reaches $1.69 \mathrm{MPa}(245 \mathrm{psia})$. The response demonstrates that the instability occurs at 
the rigid forward cylindrical mode. The instability is eliminated by increasing MB controller gains, effectively stiffening the system without reducing the damping.
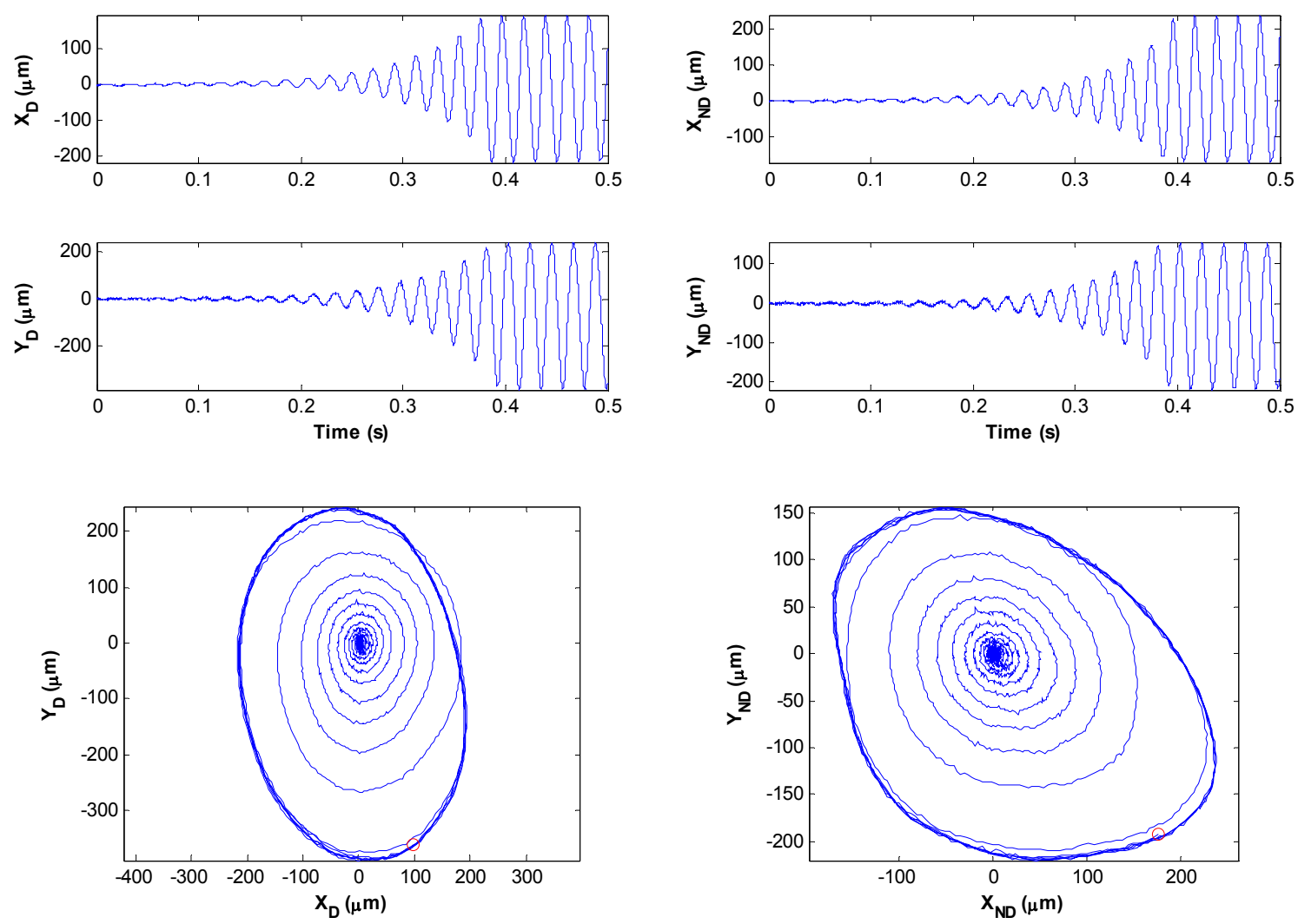

Fig. 58 Unstable system response to seal with preswirl

The final test case, $7700 \mathrm{rpm}$ with preswirl, is shown in Fig. 59 and Fig. 60. The results display a substantial degree of cross-coupling associated with the seal cross-coupled stiffness. The natural frequency has increased as a result of the gyroscopic coupling. The backward mode (visible in Fig. 54) is not observed. While the cross-coupled stiffness reduces the stability of the forward mode, it effectively dampens the backward mode. 

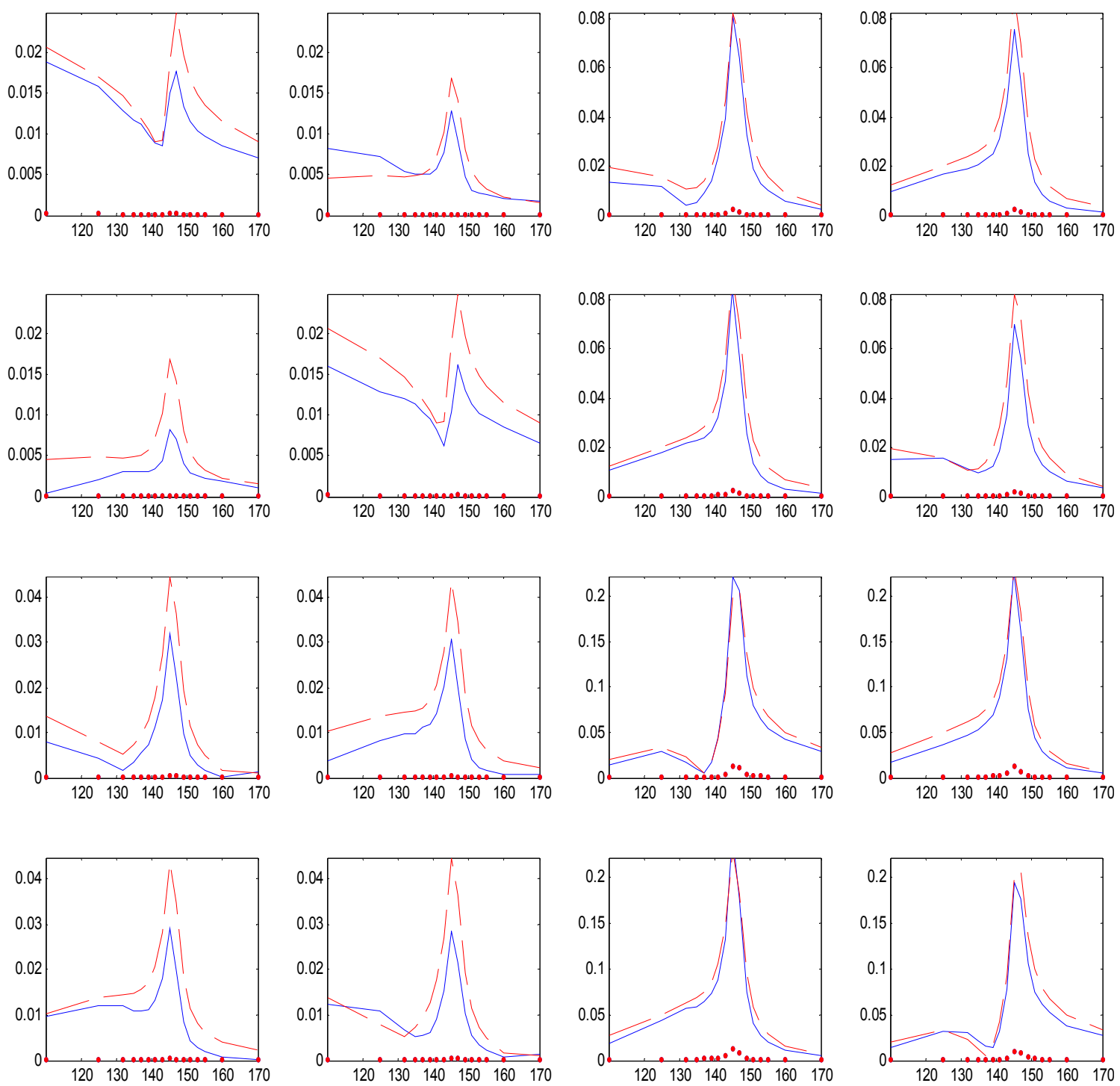

Frequency $[\mathrm{Hz}]$

Fig. 59 Frequency domain identification amplitudes $(\mu \mathrm{m} / \mathrm{N})$ at $7700 \mathrm{rpm}$ with preswirl 

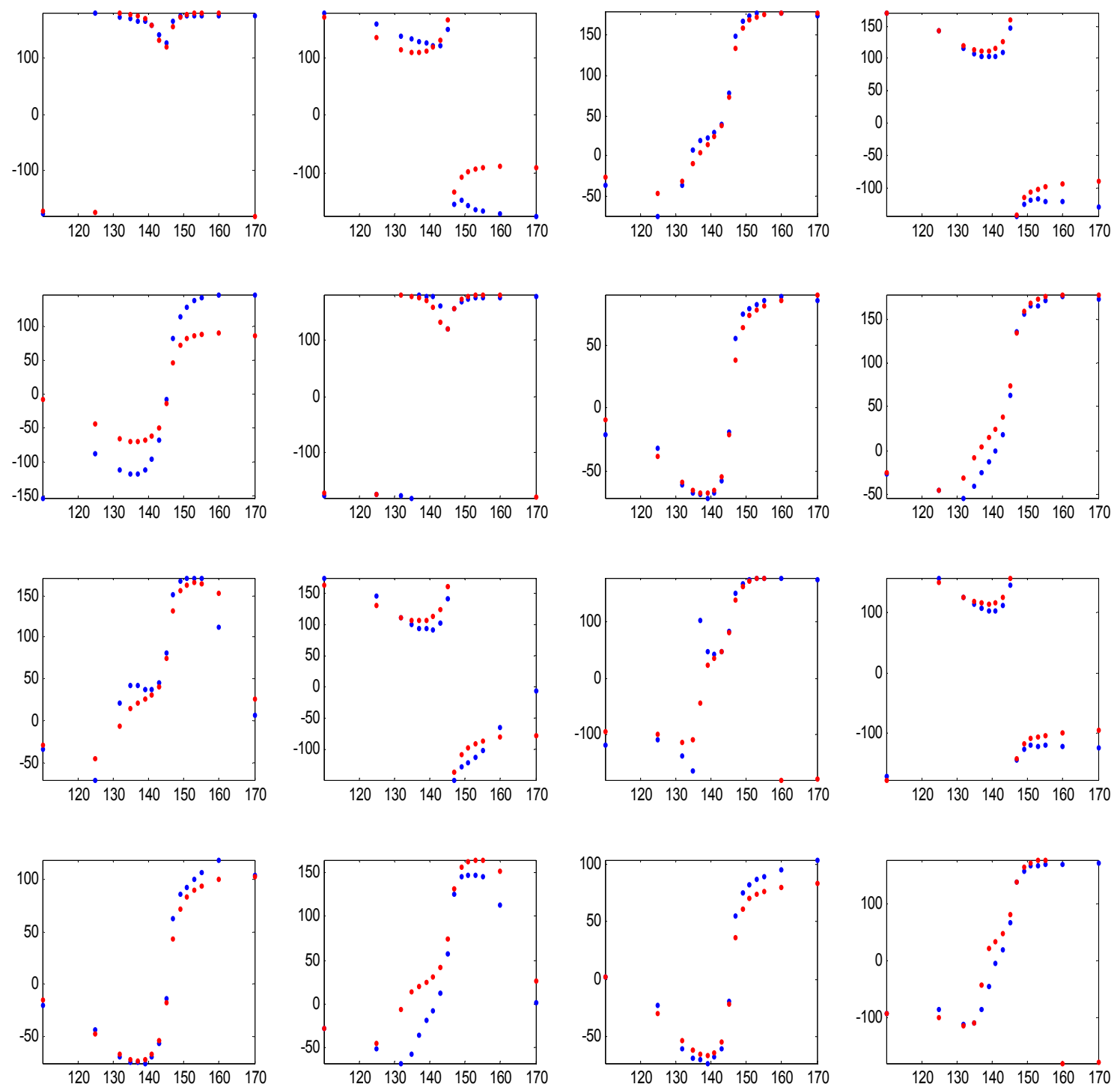

Frequency [Hz]

Fig. 60 Frequency domain identification $\left({ }^{\circ}\right)$ phases at $7700 \mathrm{rpm}$ with preswirl

Initial attempts at rotating for this configuration are problematic. Imbalance response begins to increase at $2000 \mathrm{rpm}$; at around $3000 \mathrm{rpm}$ the response prohibits any further increase in rotor speed. The large imbalance response is the result of the reduced damping of the forward cylindrical mode. The testing is accomplished by accelerating 
the rotor to the test speed (well above the cylindrical mode) and then introducing the pressurized air to the seal.

\section{Frequency Domain Identification Results and Conclusions}

The identified seal coefficients are displayed in Table 5 and Table 6 . On the first test date, the non-rotating tests consist of 10 repeated tests. All test cases are repeated on a different test date for repeatability comparison. The standard deviations for the seal coefficients are computed using a Monte Carlo simulation achieved by perturbing the DFTF results with random errors based on standard deviations from repeated tests. The force levels associated with each coefficient are computed using the excitation amplitude of $10 \mu \mathrm{m}$ and, in the case of the damping coefficient, a frequency of $140 \mathrm{~Hz}$. Force levels are included for comparison with previous uncertainty results.

Table 5 Frequency domain identification test results (metric units)

\begin{tabular}{|c|c|c|c|c|c|c|c|c|c|c|c|c|}
\hline Preswirl & Speed & Tests & Date & $K$ & K St. Dev & K Force & C & C St. Dev. & C Force & k & k St. Dev. & k Force \\
\hline- & rpm & - & - & $\mathrm{N} / \mathrm{m}$ & $\mathrm{N} / \mathrm{m}$ & $\mathrm{N}$ & $\mathrm{N}-\mathrm{s} / \mathrm{m}$ & $\mathrm{N}-\mathrm{s} / \mathrm{m}$ & $\mathrm{N}$ & $\mathrm{N} / \mathrm{m}$ & $\mathrm{N} / \mathrm{m}$ & $\mathrm{N}$ \\
\hline $\mathrm{N}$ & 0 & 10 & $12 / 2 / 2006$ & $-1.93 E+06$ & $4.17 E+04$ & 19.4 & $7.26 \mathrm{E}+03$ & $8.77 \mathrm{E}+01$ & 63.9 & - & - & - \\
\hline $\mathrm{N}$ & 0 & 1 & $2 / 2 / 2006$ & $-1.96 \mathrm{E}+06$ & $3.69 \mathrm{E}+04$ & 19.7 & $7.49 \mathrm{E}+03$ & $7.71 \mathrm{E}+01$ & 65.9 & - & - & - \\
\hline $\mathrm{N}$ & 8000 & 1 & $2 / 2 / 2006$ & $-2.00 \mathrm{E}+06$ & $2.26 \mathrm{E}+04$ & 20.0 & $8.97 E+03$ & $3.37 \mathrm{E}+01$ & 79.0 & $1.74 \mathrm{E}+06$ & $2.84 \mathrm{E}+04$ & 17.4 \\
\hline $\mathrm{N}$ & 7700 & 1 & $3 / 2 / 2006$ & $-2.09 E+06$ & $2.22 \mathrm{E}+04$ & 20.9 & $1.07 E+04$ & $4.94 \mathrm{E}+01$ & 94.3 & $2.20 E+06$ & $2.96 \mathrm{E}+04$ & 22.0 \\
\hline$Y$ & 0 & 10 & $2 / 6 / 2006$ & $-1.70 E+06$ & $2.63 \mathrm{E}+04$ & 17.0 & $7.85 \mathrm{E}+03$ & $2.34 \mathrm{E}+02$ & 69.1 & $8.18 E+06$ & $1.96 \mathrm{E}+05$ & 81.9 \\
\hline$Y$ & 0 & 1 & $2 / 8 / 2006$ & $-2.04 E+06$ & $2.05 \mathrm{E}+04$ & 20.4 & $8.11 \mathrm{E}+03$ & $2.87 \mathrm{E}+02$ & 71.4 & $7.88 \mathrm{E}+06$ & $2.65 \mathrm{E}+05$ & 78.9 \\
\hline$Y$ & 7700 & 1 & $2 / 8 / 2006$ & $-2.97 \mathrm{E}+06$ & $2.57 \mathrm{E}+04$ & 29.7 & $1.01 \mathrm{E}+04$ & $8.12 \mathrm{E}+01$ & 89.1 & $1.16 \mathrm{E}+07$ & $8.83 E+04$ & 115.7 \\
\hline $\bar{Y}$ & 7700 & 1 & $2 / 27 / 2006$ & $-2.94 E+06$ & $2.00 \mathrm{E}+04$ & 29.5 & $1.14 \mathrm{E}+04$ & $2.39 \mathrm{E}+02$ & 100.1 & $1.21 \mathrm{E}+07$ & $2.26 \mathrm{E}+05$ & 121.6 \\
\hline
\end{tabular}

Table 6 Frequency domain identification test results (English units)

\begin{tabular}{|c|c|c|c|c|c|c|c|c|c|c|c|c|}
\hline Preswirl & Speed & Tests & Date & $\mathrm{K}$ & K St. Dev & K Force & C & C St. Dev. & C Force & $\mathbf{k}$ & k St. Dev. & k Force \\
\hline- & $\mathrm{rpm}$ & - & - & Ib/in & $\mathrm{Ib} / \mathrm{in}$ & $\mathrm{lb}$ & Ib-s/in & lb-s/in & $\mathrm{Ib}$ & $\mathrm{lb} / \mathrm{in}$ & $\mathrm{lb} / \mathrm{in}$ & $\mathrm{Ib}$ \\
\hline $\mathrm{N}$ & 0 & 10 & $12 / 2 / 2006$ & -11049 & 238 & 4.4 & 41.4 & 0.50 & 14.4 & - & - & - \\
\hline $\mathrm{N}$ & 0 & 1 & $2 / 2 / 2006$ & -11218 & 211 & 4.4 & 42.8 & 0.44 & 14.8 & - & - & - \\
\hline $\mathrm{N}$ & 8000 & 1 & $2 / 2 / 2006$ & \begin{tabular}{|l|}
-11412 \\
\end{tabular} & 129 & 4.5 & 51.2 & 0.19 & 17.8 & 9922 & 162 & 3.91 \\
\hline $\mathrm{N}$ & 7700 & 1 & $3 / 2 / 2006$ & \begin{tabular}{|l|}
-11922 \\
\end{tabular} & 127 & 4.7 & 61.1 & 0.28 & 21.2 & 12565 & 169 & 4.95 \\
\hline$Y$ & 0 & 10 & $2 / 6 / 2006$ & -9718 & 150 & 3.8 & 44.8 & 1.34 & 15.5 & 46735 & 1122 & 18.41 \\
\hline$Y$ & 0 & 1 & $2 / 8 / 2006$ & -11645 & 117 & 4.6 & 46.3 & 1.64 & 16.1 & 45003 & 1514 & 17.73 \\
\hline $\bar{Y}$ & 7700 & 1 & $2 / 8 / 2006$ & -16972 & 147 & 6.7 & 57.8 & 0.46 & 20.0 & 66029 & 504 & 26.02 \\
\hline$Y$ & 7700 & 1 & $2 / 27 / 2006$ & -16806 & 114 & 6.6 & 64.9 & 1.37 & 22.5 & 69363 & 1288 & 27.33 \\
\hline
\end{tabular}


In some cases, the results from the repeated test fall within the standard deviations. In other cases this is not true; i.e. direct stiffness $K$ for the tests with preswirl at $0 \mathrm{rpm}$. This demonstrates that the changes in the results that are not explained by statistical random errors. Some possible explanations include issues with the fiber-optic strain gauges (FOSGs), changes in operating conditions, and physical changes in the system. Fig. 61 and Fig. 62 display DFTFs for tests on two different days for the case at $0 \mathrm{rpm}$ with preswirl. A slight shift in the natural frequency is noticeable and serves as an explanation for the change in the direct stiffness identification. Also, note that the difference in force levels for the identified direct stiffness coefficients from the two tests is only $3.4 \mathrm{~N}(.8 \mathrm{lb})$, which is $.1 \%$ of the bearing load capacity.

These results are consistent with uncertainty claims from the previous section. The conclusion is that the uncertainties for the frequency domain identification, at most, correspond with force levels of $.1 \%$ of load capacity. In some cases smaller uncertainties appear reasonable. The statistical uncertainty analysis indicates that, in the absence of all but random errors, uncertainties of $.01 \%$ to $.05 \%$ of load capacity are achievable. 

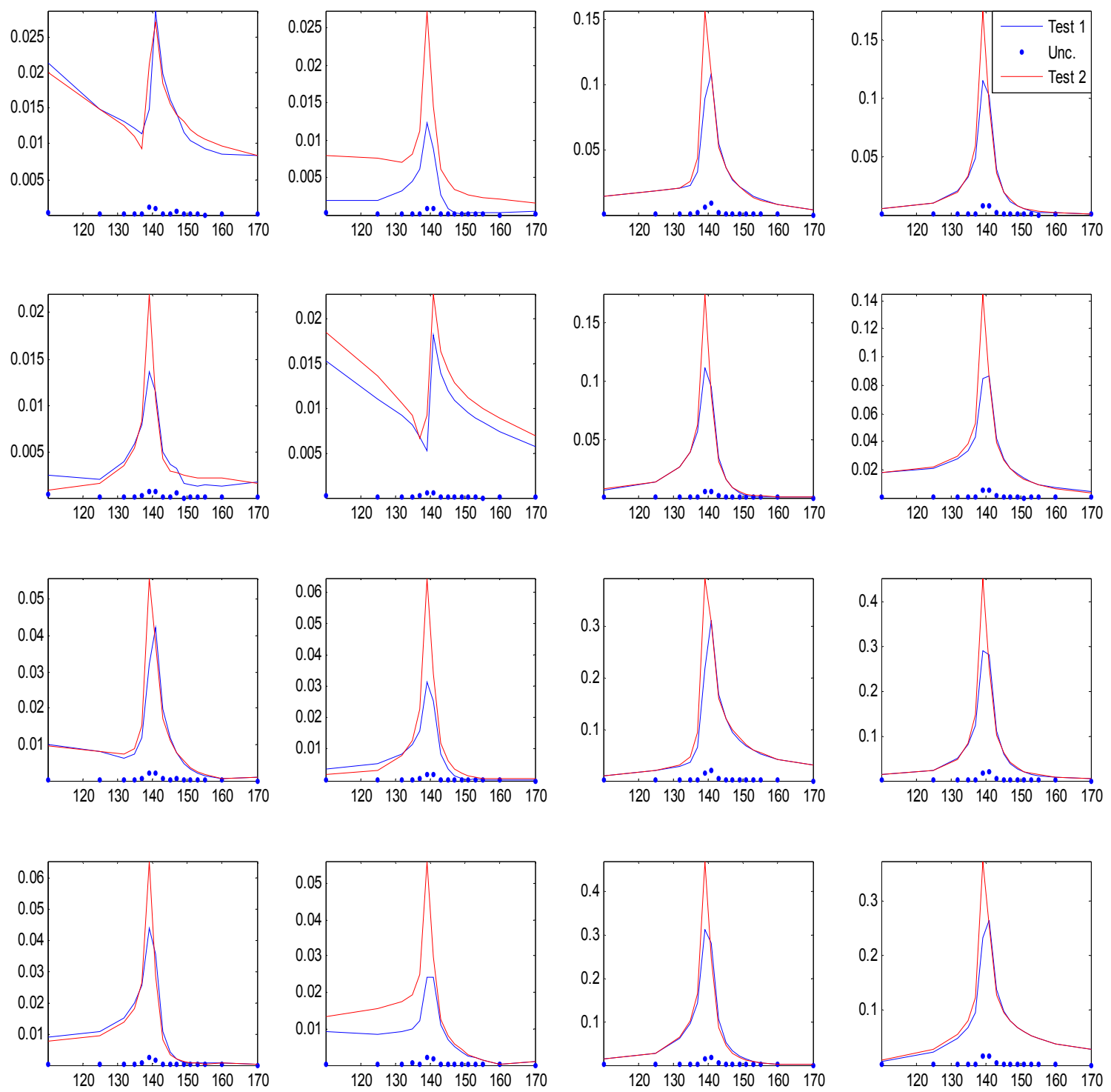

Frequency $[\mathrm{Hz}]$

Fig. 61 Comparison of DFTF amplitudes $(\mu \mathrm{m} / \mathrm{N})$ for two tests with same test conditions 

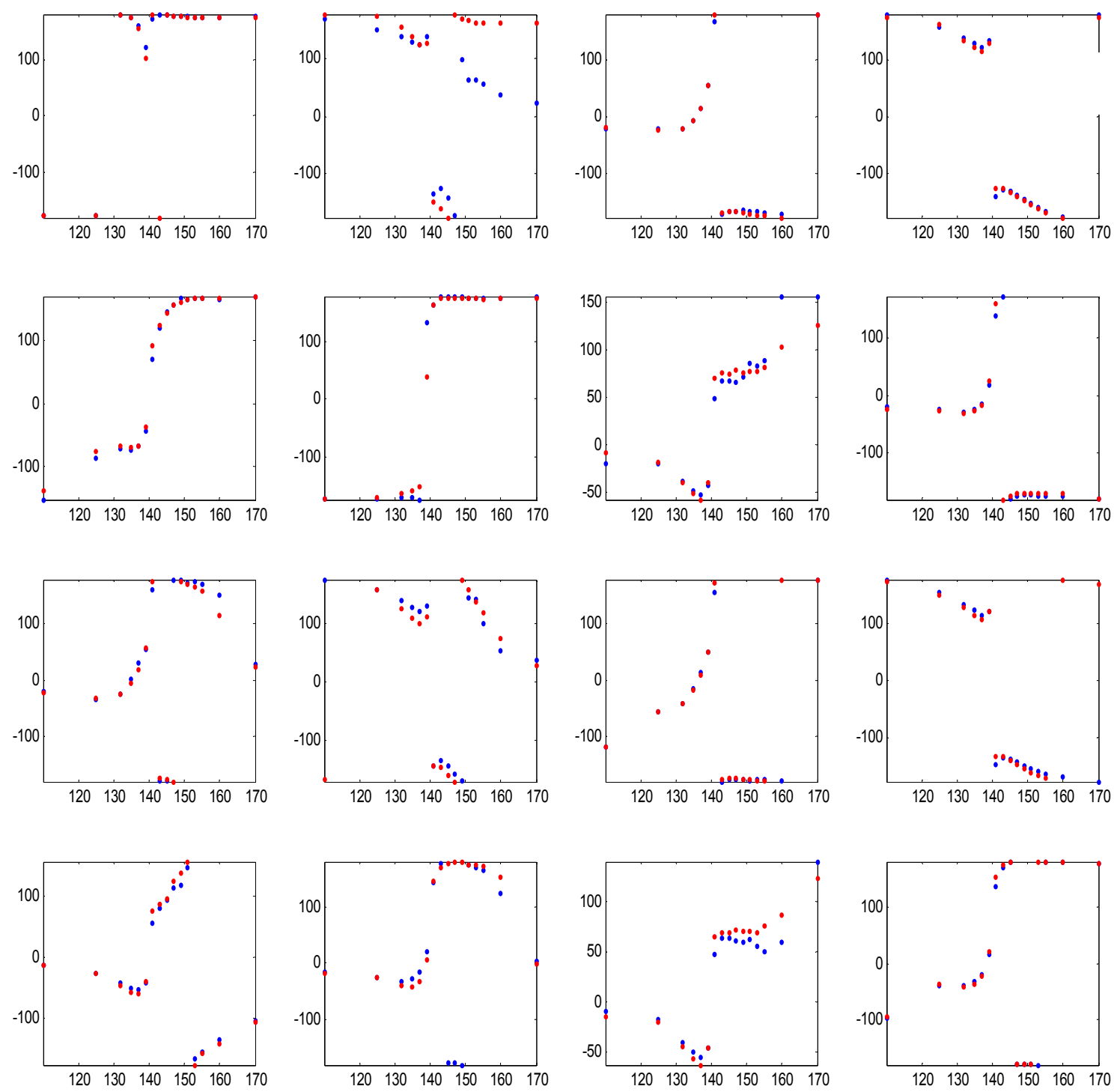

Frequency [Hz]

Fig. 62 Comparison of DFTF phases $\left(^{\circ}\right)$ for two tests with same test conditions

\section{Time Domain Identification}

The time domain identification efforts do not result in a successful determination of the seal coefficients. This section highlights the time domain methods that are attempted, and explains the reasons for the identification failure using numerical examples. While 
the identification is unsuccessful, the process yields some useful conclusion regarding time domain identification.

\section{Direct Integration Method}

One basic approach to time domain identification is to directly integrate the modeled equations of motion. Initial values for the parameters are used for the first integration, and the parameters are iterated for each subsequent iteration to minimize the error between the experimental response and the predicted response. For low order models, and derivative minimization algorithm, such as Gaussian least squares differential correction (GLSDC) [5], is well suited. For a high order system model, a non-derivative method such as the Nelder-Mead simplex method can be used to minimize the experiment-model error.

For the present experimental setup, a complication arises for the direct integration method. Predictions and frequency domain results indicate that the seal direct stiffness is negative. In addition, the rotation and preswirl both generate significant cross-coupled stiffness terms. The result is that the rigid modes of the free-free model are unstable. Table 7 shows the first twelve eigenvalues and the corresponding damped natural frequencies of the system for the different test cases. These results are obtained by using the FE system model with seal coefficients from the frequency domain identification. The table demonstrates that each case contains four low-frequency eigenvalues with positive real terms.

These unstable modes preclude the use of the direct integration approach for time domain identification. For an experimental setup, any measurement contains some level of error. For the MB test rig, there is significant measurement noise, in particular at high frequencies. While a frequency domain approach eliminates the noise at non-test frequencies by taking a FFT of the test data, the time domain approach is susceptible to noise at all frequencies. Any perturbation of the true applied force or rotor position 
excites the unstable modes. The error grows exponentially with time, rendering the direct identification method for time domain identification unsuccessful.

Table 7 Eigenvalues of system model with frequency domain identification seal coefficients

\begin{tabular}{|c|c|c|c|c|c|c|c|}
\hline \multicolumn{2}{|c|}{ 0 RPM No Preswirl } & \multicolumn{2}{c|}{ 7700 RPM No Preswirl } & \multicolumn{2}{c|}{ 0 RPM Preswirl } & \multicolumn{2}{c|}{ 7700 RPM Preswirl } \\
\hline$\omega_{\mathrm{d}}$ & $\lambda$ & $\omega_{\mathrm{d}}$ & $\lambda$ & $\omega_{\mathrm{d}}$ & $\lambda$ & $\omega_{\mathrm{d}}$ & $\lambda$ \\
\hline$(\mathrm{Hz})$ & $\left(\mathrm{s}^{-1}\right)$ & $(\mathrm{Hz})$ & $\left(\mathrm{s}^{-1}\right)$ & $(\mathrm{Hz})$ & $\left(\mathrm{s}^{-1}\right)$ & $(\mathrm{Hz})$ & $\left(\mathrm{s}^{-1}\right)$ \\
\hline 0.0 & -174.4 & 0.0 & $-22+0.1 \mathrm{i}$ & 4.1 & $32.5+26 \mathrm{i}$ & 3.7 & $-40.8+23.2 \mathrm{i}$ \\
\hline 0.0 & -174.4 & 0.0 & $-22-0.1 \mathrm{i}$ & 4.1 & $32.5-26 \mathrm{i}$ & 3.7 & $-40.8-23.2 \mathrm{i}$ \\
\hline 0.0 & 104.5 & 2.8 & $20+17.3 \mathrm{i}$ & 4.1 & $-34.3+26 \mathrm{i}$ & 6.4 & $38.7+40.4 \mathrm{i}$ \\
\hline 0.0 & 104.5 & 2.8 & $20-17.3 \mathrm{i}$ & 4.1 & $-34.3-26 \mathrm{i}$ & 6.4 & $38.7-40.4 \mathrm{i}$ \\
\hline 0.0 & -21.5 & 8.6 & $109.8+53.8 \mathrm{i}$ & 27.4 & $174.7+172.1 \mathrm{i}$ & 33.7 & $206.8+211.9 \mathrm{i}$ \\
\hline 0.0 & -21.5 & 8.6 & $109.8-53.8 \mathrm{i}$ & 27.4 & $174.7-172.1 \mathrm{i}$ & 33.7 & $206.8-211.9 \mathrm{i}$ \\
\hline 0.0 & 19.9 & 9.1 & $-196.8+56.9 \mathrm{i}$ & 29.8 & $-252.9+187.3 \mathrm{i}$ & 38.3 & $-308.1+240.8 \mathrm{i}$ \\
\hline 0.0 & 19.9 & 9.1 & $-196.8-56.9 \mathrm{i}$ & 29.8 & $-252.9-187.3 \mathrm{i}$ & 38.3 & $-308.1-240.8 \mathrm{i}$ \\
\hline 139.5 & $-29.3+876.7 \mathrm{i}$ & 131.5 & $-39+826.3 \mathrm{i}$ & 138.2 & $-69.2+868.2 \mathrm{i}$ & 128.6 & $-83.6+807.9 \mathrm{i}$ \\
\hline 139.5 & $-29.3-876.7 \mathrm{i}$ & 131.5 & $-39-826.3 \mathrm{i}$ & 138.2 & $-69.2-868.2 \mathrm{i}$ & 128.6 & $-83.6-807.9 \mathrm{i}$ \\
\hline 139.5 & $-29.3+876.7 \mathrm{i}$ & 145.8 & $-31+916.4 \mathrm{i}$ & 139.8 & $3.5+878.7 \mathrm{i}$ & 145.6 & $10.9+914.7 \mathrm{i}$ \\
\hline 139.5 & $-29.3-876.7 \mathrm{i}$ & 145.8 & $-31-916.4 \mathrm{i}$ & 139.8 & $3.5-878.7 \mathrm{i}$ & 145.6 & $10.9-914.7 \mathrm{i}$ \\
\hline
\end{tabular}

In an effort to eliminate the unstable modes from the system model, the model is reduced using the built-in Matlab function modreal. This function produces a slow realization and fast realization of the system, separated at the specified number of eigenvalues. Fig. 63 and Fig. 64 compare the DFTFs of the fast realization (all unstable modes are eliminated) to the full order model DFTFs in the frequency range of the first bending mode. There are substantial differences between the responses of the two systems. The attempt to eliminate the unstable modes while preserving the behavior of the system at the other modes is unsuccessful. A method that can accomplish time domain identification of a system with unstable modes is required. 

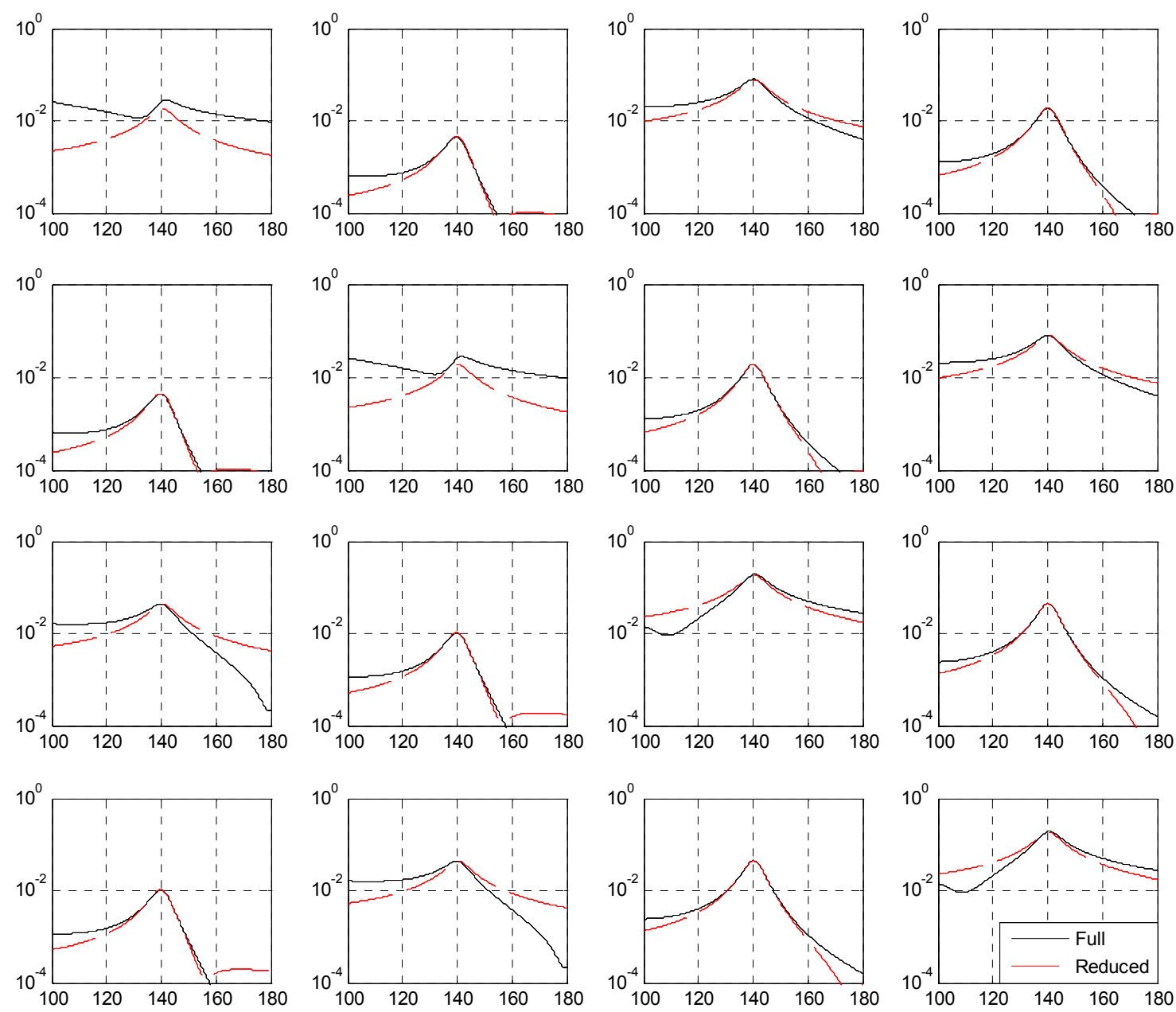

Frequency $[\mathrm{Hz}]$

Fig. 63 Comparison of DFTF amplitudes $(\mu \mathrm{m} / \mathrm{N})$ for full and reduced system models 

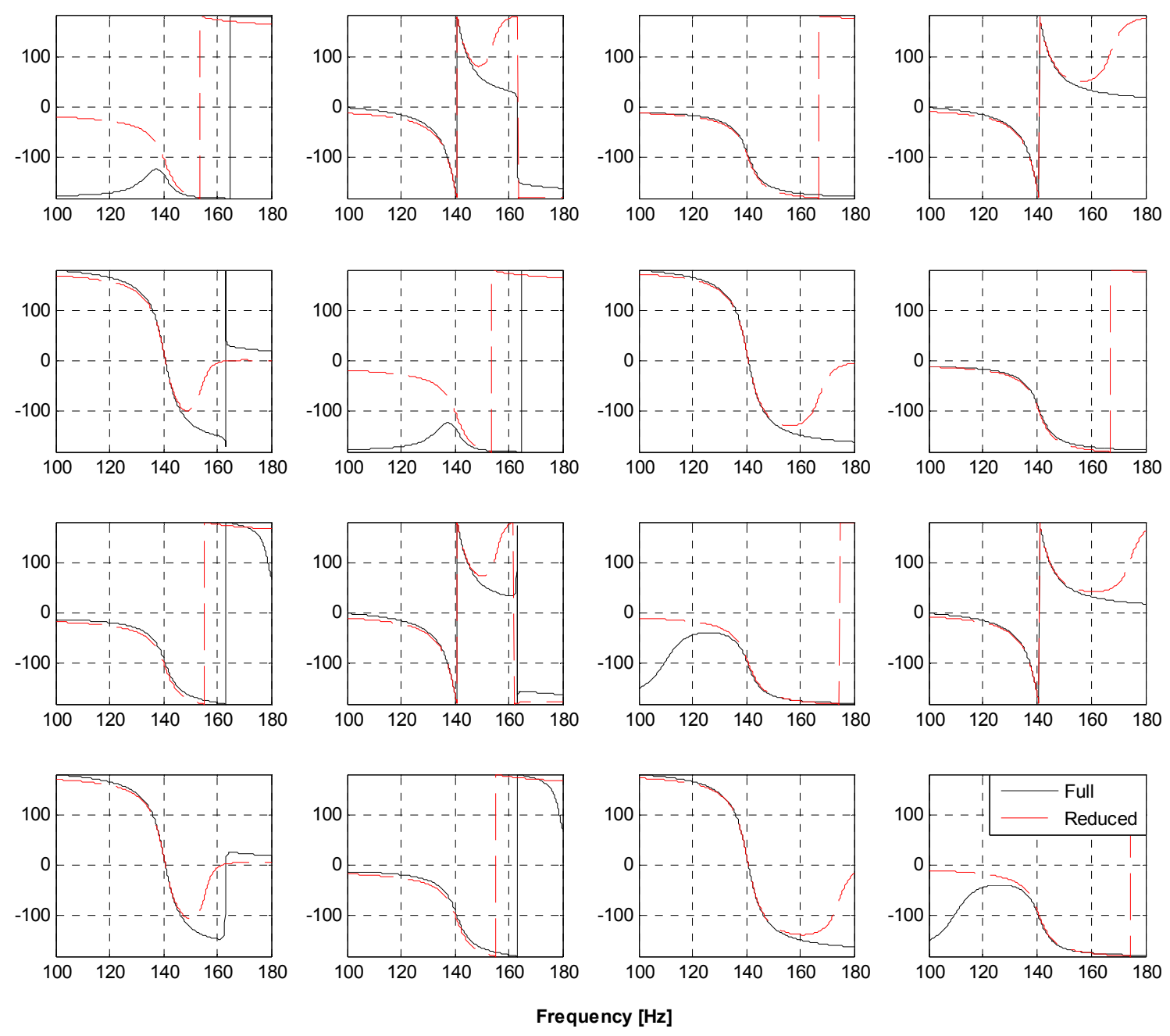

Fig. 64 Comparison of DFTF phases $\left({ }^{\circ}\right)$ for full and reduced system models

\section{Observer/Kalman Filter Identification with ERA}

Another approach to the time domain identification problem uses a discrete system model and the system response to identify the system. The system response is characterized by sampled pulse system response histories know as system Markov parameters. In cases where the system has lightly damped or unstable modes, the system model can be augmented to include a stable observer. The observer Markov parameters are identified, and a system realization can be computed from the Markov parameters 
with a procedure known as the Eigensystem Realization Algorithm (ERA). This process is explained in detail in the text by Juang [6].

The discrete time system model is given in Eq. (37). The discrete state matrix $A_{d}$ is computed from the time step $\Delta t$ and the continuous state matrix $A$ using a Taylor series expansion. In practice, more efficient numerical methods are used to approximate the Taylor series expansion. The discrete input matrix is computed using the discrete and continuous state matrices and the continuous input matrix $B$. The output and feedthrough matrices are unchanged [6]. In practice, there are more numerically efficient and robust ways to compute the approximate discrete state matrix [32].

$$
\begin{aligned}
& x(k+1)=A_{d} x(k)+B_{d} u(k) \\
& y(k)=C x(k)+D u(k) \\
& A_{d}=e^{A \Delta t}=I+A \Delta t+\frac{1}{2 !}(A \Delta t)^{2} \ldots \\
& B_{d}=\int_{0}^{\Delta t} e^{A \Delta \tau} d \tau B=\left(A_{d}-I\right) A^{-1} B
\end{aligned}
$$

The state variables can be eliminated from the equations. This solution assumes zero initial conditions for the states.

$$
\begin{aligned}
y & =Y U \\
y & =\left[\begin{array}{llll}
y(0) & y(1) & \cdots & y(n-1)
\end{array}\right] \\
Y & =\left[\begin{array}{lllll}
D & C B_{d} & C A_{d} B_{d} & \cdots & C A_{d}{ }^{n-2} B_{d}
\end{array}\right] \\
U & =\left[\begin{array}{cccc}
u(0) & u(1) & \cdots & u(n-1) \\
& u(0) & & \vdots \\
& & \ddots & u(1) \\
& & & u(0)
\end{array}\right]
\end{aligned}
$$


The parameters given in matrix $Y$ are the Markov parameters. The number of Markov parameters can be reduced by truncating the $Y$ and $U$ matrices. This is only a reasonable approximation if the system is well damped.

$$
\begin{aligned}
y & =\tilde{Y} \tilde{U} \\
\tilde{Y} & =\left[\begin{array}{llllll}
D & C B_{d} & C A_{d} B_{d} & \cdots & C A_{d}{ }^{p-1} B_{d}
\end{array}\right] \\
\tilde{U} & =\left[\begin{array}{cccccc}
u(0) & u(1) & & \cdots & u(n-1) \\
& u(0) & & & \vdots \\
& & \ddots & & & \\
& & & u(0) & \cdots & u(n-p-1)
\end{array}\right]
\end{aligned}
$$

In the case of lightly damped systems, an observer gain matrix can be added to improve the system stability [33]. The system and input matrices for the augmented system are displayed below in Eq. (40).

$$
\begin{aligned}
& \bar{A}=A_{d}+G C \\
& \bar{B}=\left[B_{d}+G D,-G\right] \\
& v(k)=\left[\begin{array}{l}
u(k) \\
y(k)
\end{array}\right]
\end{aligned}
$$

The Markov parameters for the augment system are known as observer Markov parameters. The states can again be eliminated from the equations with a slight modification from Eq. (39). 


$$
\begin{aligned}
& y=\bar{Y} \bar{V} \\
& \bar{Y}=\left[\begin{array}{lllll}
D & C \bar{B} & C \bar{A} \bar{B} & \cdots & C \bar{A}^{p-1} \bar{B}
\end{array}\right]
\end{aligned}
$$

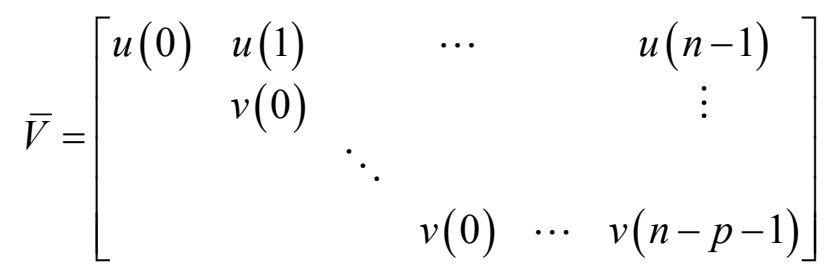

The observer Markov parameters can be determined with the least squares solution or using the pseudo-inverse $\bar{V}^{+}$shown below in Eq. (42). The pseudo-inverse must be used if $\bar{V} \bar{V}^{T}$ is rank deficient.

$$
\begin{gathered}
\bar{Y}=y \bar{V}^{T}\left(\bar{V} \bar{V}^{T}\right)^{-1} \\
\bar{Y}=y \bar{V}^{+}
\end{gathered}
$$

The identified observer gain is in fact the Kalman filter gain for the appropriate process and measurement noise statistics [6]. For this reason, this identification approach is known as Observer/Kalman Filter Identification (OKID). By augmenting the system, systems with lightly damped and unstable modes can be identified.

Once the observer Markov parameters are determined, a procedure known as the Eigensystem Realization Algorithm can be used to determine the state-space matrices [34]. This procedure assembles the Markov parameters into a Hankel matrix, which is then decomposed using singular value decomposition. By analyzing the singular values, the significant modes are determined and a minimal realization is obtained. The ERA is explained in detail in the text by Juang [6]. The minimal realization is obtained and transformed into modal coordinates. These results can then be compared with predictions from the FE model to determine the seal coefficients. This time domain identification procedure is summarized below. 
1. Markov parameters are identified using the method described above from the time domain experimental data.

2. Natural frequencies, damping ratios, and mode shapes are determined from the Markov parameters using the Eigensystem Realization Algorithm.

3. Identification results are compared with predictions from the identification system FE model to obtain the seal coefficients.

Unfortunately, attempts to perform time identification using this method are unsuccessful. This method can accommodate the system instabilities; however, there are numerical complications and test rig limitations that prevent this method from determining the seal coefficients. A simple example is developed in an effort to explain the problems encountered with OKID.

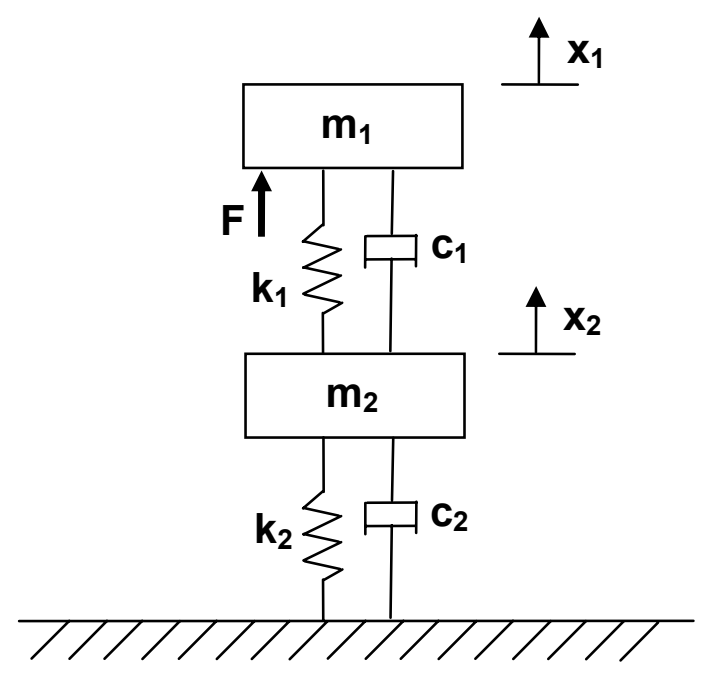

Fig. 65 Two degree-of-freedom OKID example

The model shown in Fig. 65 is used to develop two systems with parameters displayed in Table 8. The first system has two natural frequencies with the second $(180 \mathrm{~Hz})$ roughly twice the frequency of the first $(89 \mathrm{~Hz})$. The second system has a significantly lower natural frequency; the first natural frequency is $2.9 \mathrm{~Hz}$, and the second is $123 \mathrm{~Hz}$. This 
is accomplished by changing the value of $k_{2}$. The second system is representative of the MB test rig. Referring to Table 7, the low frequency eigenvalues of the identification system model range from 0 to $40 \mathrm{~Hz}$, with most below $10 \mathrm{~Hz}$. The first bending mode natural frequencies are around $140 \mathrm{~Hz}$.

\section{Table 8 System parameters for OKID example}

\begin{tabular}{|c|c|c|}
\hline & System 1 & System 2 \\
\hline $\mathbf{m}_{\mathbf{1}}$ & 1 & 1 \\
\hline $\mathbf{m}_{\mathbf{2}}$ & 2 & 2 \\
\hline $\mathbf{k}_{\mathbf{1}}$ & 40000 & 40000 \\
\hline $\mathbf{k}_{\mathbf{2}}$ & 2000000 & 1000 \\
\hline $\mathbf{c}_{\mathbf{1}}$ & 100 & 100 \\
\hline $\mathbf{c}_{\mathbf{2}}$ & 30 & 30 \\
\hline
\end{tabular}

Both systems are identified for two cases, with and without noise. The excitation signal consists of random numbers normally distributed about zero with unit standard deviation. For the cases with noise, normally distributed random noise is added to the true measurements. The standard deviation of the noise is calculated to be $1 \%$ of the measurement standard deviation. In all cases, the sample frequency, number of samples, and size of the sampled pulse system response matrix $\bar{V}$ are adjusted to produce the best possible identification results.

The true and identified natural frequencies and damping ratios for each system are displayed in Table 9. For the identification of the first system without measurement noise, the identified natural frequencies and damping ratios very nearly match the true values. With the addition of noise, natural frequencies are identified to within $2 \%$ of the true values; damping ratios are identified with errors of $73 \%$ and $4.8 \%$ respectively. These results are consistent with examples from the text by Juang [6] for systems with lightly damped lower frequencies. These errors might still be acceptable for identification; however, this problem worsens for the case with a very low first natural frequency. The characteristics of the second system are identified reasonably well for 
the case without noise. The addition of noise precludes any reasonable identification of the first natural frequency. The identified frequency is 5.6 times larger than the true value, and the identified damping ratio is 2.3 times greater than the true damping ratio.

Table 9 Natural frequencies and damping ratios from OKID example

\begin{tabular}{|l|c|c|c|c|}
\hline \multicolumn{1}{|c|}{ Case } & Freq. 1 & Damp \% & Freq 2 & Damp \% \\
\hline Sys. 1- True Values & 88.8379 & 5.0296 & 180.1234 & 4.8034 \\
\hline Sys. 1- No Measurement Noise & 88.8377 & 5.0381 & 180.1231 & 4.8035 \\
\hline Sys. 1- 1\% Measurement Noise & 87.3656 & 8.7227 & 176.6311 & 5.0267 \\
\hline Sys. 2- True Values & 2.9055 & 27.3759 & 123.2930 & 10.0045 \\
\hline Sys. 2- No Measurement Noise & 2.9080 & 27.3200 & 123.2930 & 10.0045 \\
\hline Sys. 2- 1\% Measurement Noise & 16.1830 & 63.9848 & 124.7228 & 10.3500 \\
\hline
\end{tabular}

The problem associate with the large natural frequency separation prevents any reasonable identification of seal parameters. If the example system masses are determined prior to identification and treated as know values, the error associated with the first natural frequency would result in an over-estimation of stiffness $k_{2}$ by a factor of roughly 30. As shown previously in Fig. 63 and Fig. 64 the low frequency modes cannot be eliminate from the system model, preventing an attempt to avoid the adverse effects of the low frequency modes.

The problems associated with the low natural frequencies are further compound by the fact that the system is difficult to excite at low frequencies. Displacements at low frequencies require very little force. The FOSG calibration is only valid to excitation amplitudes to $20 \mu \mathrm{m}$ (.00079 in). A sinusoidal excitation of this amplitude at $5 \mathrm{~Hz}$ requires a force amplitude of only $4.3 \mathrm{~N}\left(1.0 \mathrm{lb}_{\mathrm{f}}\right)$. This force amplitude is on the order of the FOSG measurement uncertainty. The result is that the force measurements from the FOSGs contain much more noise than $1 \%$ noise levels used in the previous example.

Rotordynamic systems typically have inherent cyclic responses that are deleterious to experimental measurements. Imbalance response is a cyclic vibration of the rotor that 
occurs as a result of imperfections in the mass distribution of the rotor. From an identification stand point, the system appears to be vibrating without any excitation force. Another cyclic property is the motion probe runout. Imperfections in the rotor surface produce measurement in the motion probes that do not reflect the true position of the rotor. These effects combined with the large natural frequency separations and the high noise levels provide a suitable explanation for the failure of this identification method.

The time domain identification methods attempted on the MB test rig failed to obtain identified seal coefficients for a variety of factors. The following list highlights the major characteristics of the test rig and air seal that prevent effective time domain identification, using the algorithms considered:

- The seal forces, specifically negative direct stiffness and significant crosscoupled stiffness, result in an unstable system configuration for identification.

- Seal and rotor characteristics produce a large separation of system natural frequencies between rigid and flexible rotor modes.

- Excitation amplitude limitations result in low force levels and high signal to noise ratios for force measurements at low frequencies.

- Imbalance response and runout produce cyclic sources of noise that affect the identification results.

Time-domain methods encountered significant challenges that were avoided by frequency domain methods. Frequency-domain methods were advantageous because of their ability to focus on a narrow frequency range and eliminate the adverse effects of measurement noise. The final approach to determining the seal coefficients, using static measurements, is addressed in the following section. 


\section{Static Measurements}

Seal coefficients are also determined using an alternate method of force measurement for comparison. The MB forces can be calculated using an empirical current and position based formula. Results found in literature for this method are discussed previously $[11,12]$. This method does not use FOSGs and will serve as a comparison for the previous results.

The force applied by an MB can be modeled by the control currents from the top and bottom poles, $I_{\text {top }}$ and $I_{b o t t o m}$, and the location of the rotor $x$. The gap parameter $g$ represents the air gap between the $\mathrm{MB}$ poles and the rotor. The tare force $F_{0}$ is used to zero the applied force $F$.

$$
F=C\left[\left(\frac{I_{\text {top }}}{(g-x)}\right)^{2}-\left(\frac{I_{\text {bottom }}}{(g+x)}\right)^{2}\right]-F_{0}
$$

This model is calibrated by applying a known load to the rotor. The force is applied at two know axial locations (the axial center each disc show in Fig. 9), and the force applied to each MB is calculated statically. The force is applied in the horizontal and vertical directions. Current and position measurements are obtained when the rotor is initially centered, displaced horizontally by $14 \mu \mathrm{m}$ (.00056 in), and displaced vertically by $14 \mu \mathrm{m}$ (.00056 in). Data sets consist of 10 measurement averages $(.1 \mathrm{~s}$ window with 1 s spacing) taken by the MB controller. 5 data sets are taken at each calibration configuration

With a calibrated model, the rotor is displaced, and the measured force is used to compute stiffness coefficients. The force is computed with the rotor centered and at two displaced locations, horizontal and vertical. The measurements were taken for three test configurations: (i) $0 \mathrm{rpm}$ without preswirl, (ii) $0 \mathrm{rpm}$ with preswirl, (iii) $7700 \mathrm{rpm}$ with preswirl. The change in force is used to compute direct and cross-coupled stiffness 
coefficients. This is accomplished by dividing the net force components by the displacements. Table 10 displays the rotor location and configuration for the formula calibration and static seal tests.

Table 10 Static measurement test matrix

\begin{tabular}{|c|c|c|}
\hline Type & Configuration & Rotor Location \\
\hline Calibration & Disc 1 Horizontal & Centered \\
\hline Calibration & Disc 1 Horizontal & Horizontal Displacement \\
\hline Calibration & Disc 1 Horizontal & Vertical Displacement \\
\hline Calibration & Disc 1 Vertical & Centered \\
\hline Calibration & Disc 1 Vertical & Horizontal Displacement \\
\hline Calibration & Disc 1 Vertical & Vertical Displacement \\
\hline Calibration & Disc 2 Horizontal & Centered \\
\hline Calibration & Disc 2 Horizontal & Horizontal Displacement \\
\hline Calibration & Disc 2 Horizontal & Vertical Displacement \\
\hline Calibration & Disc 2 Vertical & Centered \\
\hline Calibration & Disc 2 Vertical & Horizontal Displacement \\
\hline Calibration & Disc 2 Vertical & Vertical Displacement \\
\hline Seal Test & 0 RPM, No Preswirl & Centered \\
\hline Seal Test & 0 RPM, No Preswirl & Horizontal Displacement \\
\hline Seal Test & 0 RPM, No Preswirl & Vertical Displacement \\
\hline Seal Test & 0 RPM, Preswirl & Centered \\
\hline Seal Test & 0 RPM, Preswirl & Horizontal Displacement \\
\hline Seal Test & 0 RPM, Preswirl & Vertical Displacement \\
\hline Seal Test & 7700 RPM, Preswirl & Centered \\
\hline Seal Test & 7700 RPM, Preswirl & Horizontal Displacement \\
\hline Seal Test & 7700 RPM, Preswirl & Vertical Displacement \\
\hline
\end{tabular}

\section{Results}

The static test results are displayed below in Table 11. Typical static measurement uncertainties (.1\% of load capacity per axis) specified by SKF Magnetic Bearings Inc. are used to calculate specified coefficient uncertainties. A comparison with the frequency domain identification results is used to estimate the true uncertainties. The specified uncertainties are questionable because they are valid for zero eccentricity measurements. 
Table 11 Static test results

\begin{tabular}{|c|c|c|c|c|c|}
\hline \multicolumn{2}{|c|}{} & \multicolumn{2}{c|}{ Metric Units } & \multicolumn{2}{c|}{ English Units } \\
\hline Preswirl & Speed & $\mathbf{K}$ & $\mathbf{k}$ & $\mathbf{K}$ & $\mathbf{k}$ \\
\hline- & $\mathbf{r p m}$ & $\mathbf{N} / \mathbf{m}$ & $\mathbf{N} / \mathbf{m}$ & $\mathbf{l b} / \mathbf{i n}$ & $\mathbf{I b} / \mathbf{i n}$ \\
\hline $\mathrm{N}$ & 0 & $-9.83 \mathrm{E}+05$ & - & $-5.61 \mathrm{E}+03$ & - \\
\hline $\mathrm{Y}$ & 0 & $4.65 \mathrm{E}+06$ & $1.81 \mathrm{E}+07$ & $2.66 \mathrm{E}+04$ & $1.03 \mathrm{E}+05$ \\
\hline $\mathrm{Y}$ & 7700 & $8.24 \mathrm{E}+05$ & $1.61 \mathrm{E}+07$ & $4.71 \mathrm{E}+03$ & $9.22 \mathrm{E}+04$ \\
\hline Specified Uncertanties (e=0): & $1.0 \mathrm{E}+06$ & $1.0 \mathrm{E}+06$ & $5.7 \mathrm{E}+03$ & $5.7 \mathrm{E}+03$ \\
\hline \multicolumn{2}{|r|}{ Estimated Uncertanties: } & $5.0 \mathrm{E}+06$ & $5.0 \mathrm{E}+06$ & $2.9 \mathrm{E}+04$ & $2.9 \mathrm{E}+04$ \\
\hline
\end{tabular}

The direct stiffness measurements are inaccurate. In two cases the measurements are on the same level as the specified zero eccentricity uncertainty. In the other case, the direct stiffness has the opposite sign from frequency domain identification results. This suggests that the uncertainties for the static measurements with non-zero eccentricities are a minimum of 5 times greater than those specified for zero eccentricities. The crosscoupled stiffness forces are substantially larger and, as a result, the static measurements of these coefficients are valid. The signs of the cross-coupled stiffness coefficients are correct, and the magnitudes are comparable to the frequency domain results considering the estimated uncertainty levels.

Static test results have uncertainties that are at least 10 times greater the results from the frequency domain identification. Primary factor responsible for this difference is the method of force measurement. The FOSGs bypass the need for a theory to convert from MB current and rotor position to force. The capability of FOSGs to directly measure forces applied to the rotor produces a substantial improvement in the usage of MBs as calibrated exciters.

\section{Comparisons with Predictions and Historical Testing}

The frequency domain identification coefficients are compared with predictions from the Turbomachinery Laboratory seal coefficient code XLIsotSl and with historical test data from another test rig. Both comparisons verify that results from the frequency domain identification are reasonable. 


\section{XLIsotSl}

Fig. 66 displays predictions from XLIsotSl for the various test conditions. The coefficients values are plotted against the pressure drop $(\Delta \mathrm{P})$ across the seal. The pressure drop for the experimental results is unknown, but is assumed to be the inlet gauge pressure for plotting purposes. The negative direct stiffness terms indicate that the air seal leakage predictions are incorrect and that the air flow is choked in the seal.
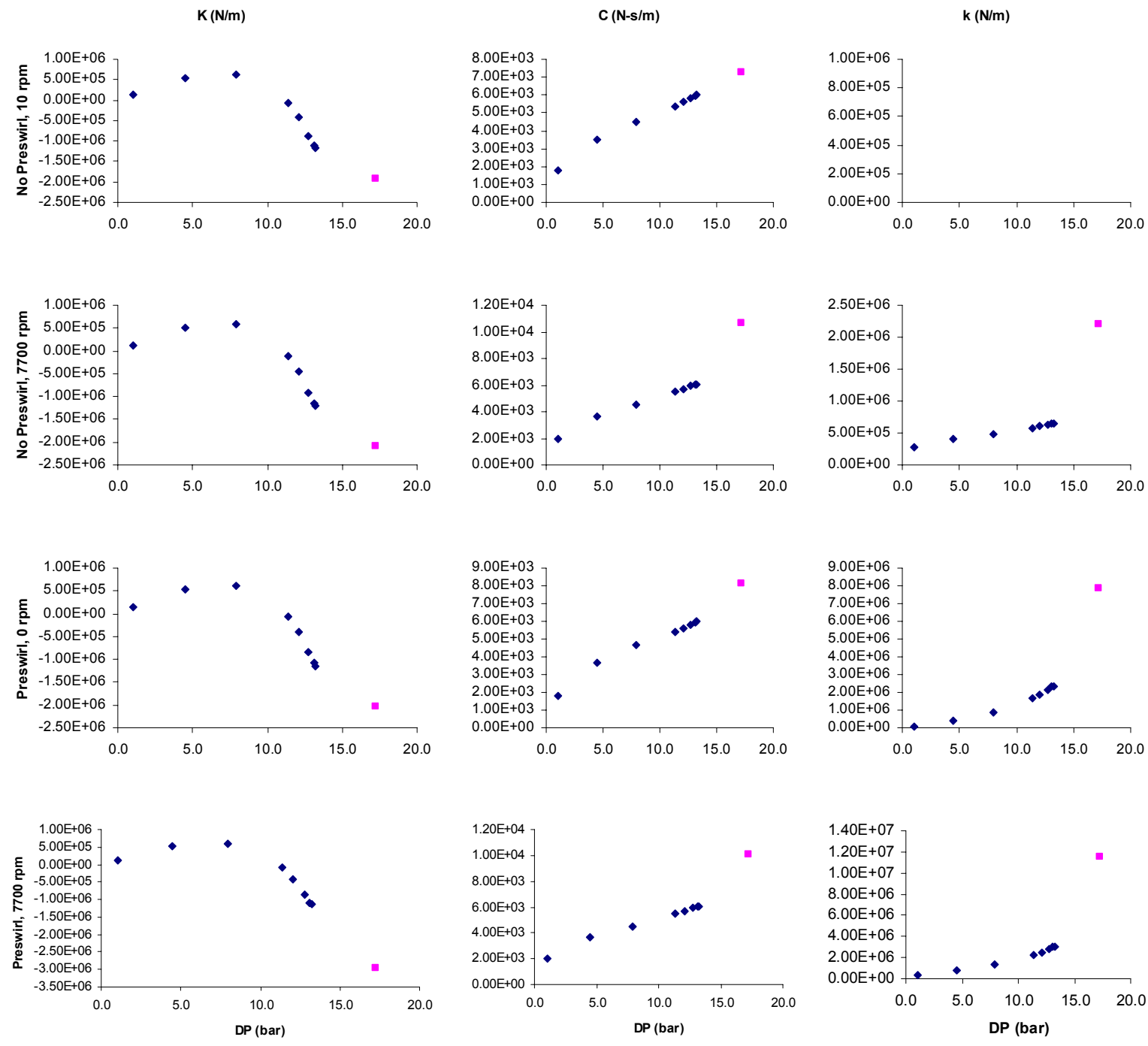

Fig. 66 Comparison of frequency domain test results with XLIsotSl predictions 
The XLIsotSL predictions are limited to air flow below the choked condition. The predictions produce a reasonable comparison with the test results. The predicted coefficients, in all cases, approach the test results as the pressure drop increases. The direct stiffness initially increases, but then decreases to a negative value, which correlates with test results. Cross-coupled stiffness values are under predicted. This is likely because preswirl values were calculated, rather than measured, based on the calculated air flow rate and the geometry of the swirl races. Overall, the predictions agree reasonably well with experimental results.

\section{Comparison with Results from Air Seal Test Rig}

Another test rig at the Turbomachinery Laboratory is specifically designed for testing high pressure air seals. This test rig measured seal coefficients for a variety of seals, including annular gas seals. Childs and Kerr [25] tested smooth seals with variables of preswirl, rotor speed, and backpressure. The cases that are most comparable to the conditions of the current research are at 10,200 rpm with $30 \%$ back pressure. The inlet pressure is 17.9 bar (260 psia) and both zero and medium preswirl results are available. Test results are non-dimensionalized and normalized according to Eq. (44) through (46). The constants are the seal radial clearance $C_{r}$, the pressure drop $\Delta P$, the seal diameter $D_{s}$, and the seal length $L$.

$$
\begin{gathered}
K_{N D}=K\left(\frac{C_{r}}{\Delta P \cdot D_{s} \cdot L}\right) \\
C_{N}=C\left(\frac{C_{r}}{\Delta P \cdot D_{s} \cdot L}\right) \\
k_{N D}=k\left(\frac{C_{r}}{\Delta P \cdot D_{s} \cdot L}\right)
\end{gathered}
$$


The non-dimensionalized and normalized results for both test rigs are listed in Table 12. The damping coefficients are very similar. Damping coefficient have a strong agreement because the inlet pressures for both test rigs were comparable, and damping coefficient are fairly independent of the rotor speed and preswirl. The cross-coupled stiffness measurements vary substantially for the no preswirl cases. This is likely because force levels associate with the cross-coupled stiffness are low for the test configurations without preswirl. The cross-coupled stiffness coefficients are comparable for cases with preswirl. The direct stiffness measurements for the air seal test were indistinguishable.

Table 12 Comparison of test results with historical results

\begin{tabular}{|c|c|c|c|c|c|c|}
\hline & \multicolumn{3}{|c|}{ Rotation, No Preswirl } & \multicolumn{3}{c|}{ Rotation, Preswirl } \\
\cline { 2 - 7 } & $\mathrm{K}(-)$ & $\mathrm{C}(\mathrm{s})$ & $\mathrm{k}(-)$ & $\mathrm{K}(-)$ & $\mathrm{C}(\mathrm{s})$ & $\mathrm{k}(-)$ \\
\hline MB Rig Tests & -0.039 & 0.00020 & 0.041 & -0.055 & 0.00019 & 0.21 \\
\hline Air Seal Rig Tests & - & 0.00017 & 0.025 & - & 0.00022 & 0.17 \\
\hline
\end{tabular}

The comparison with other test results indicates that the results from the present research are reasonable. This comparison also demonstrates that direct stiffness measurements can potentially be measured more accurately on a flexible rotor test rig because of the increased sensitivity to the direct stiffness at the first bending mode. This occurs because the location of the test seal is at the midspan of the rotor, where the maximum displacement occurs for the first bending mode. 


\section{MAGNETOSTRICTION}

Throughout the research process, the understanding of the mechanism causing the MB strain has developed. An intuitive assessment of the MB mechanics might lead one to conclude that the MB poles are in tension, based on the fact that the poles pull, rather than push, the rotor. However, this is assessment is false. The strain occurs as a result of the magnetic field, and it is, in fact, a compressive stress that produces the strain. Considering two permanent magnets (Fig. 67), if the magnets are positioned with a north pole aligned with a south pole, the magnets will pull together. The net forces acting on each magnet, $F_{1}$ and $F_{2}$, are compressive forces acting on the interface between the two magnets. Recognizing that each magnet could, itself, be divided into many smaller magnets with the orientation shown below reveals that the entire magnet is in compression as a result of the magnetic field.

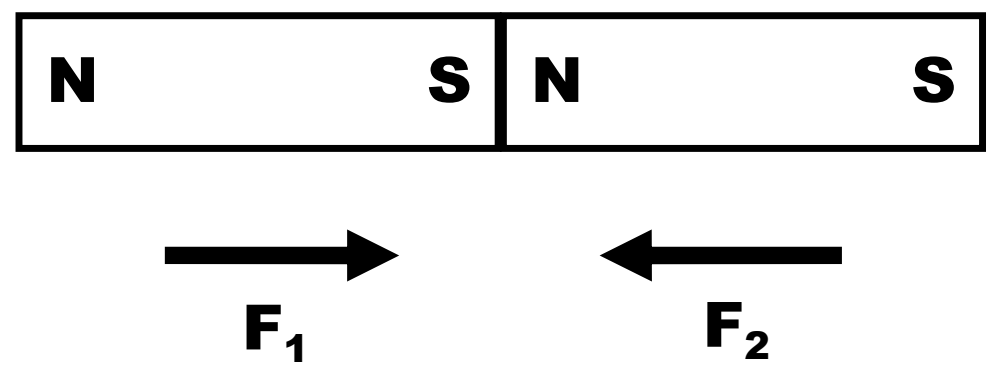

Fig. 67 Permanent magnet example of compressive forces

This analogy applies to paramagnetic materials as well. In a paramagnetic material, an externally applied magnetic field is increased within the material by the alignment of magnetic dipoles. The dipoles have a north to south orientation, resulting in a compressive stress and strain. The compressive strain that occurs in a material as a result of a magnetic field is known as magnetostriction. This section develops the theory behind magnetostriction. A FE model of the MB magnetic field is also developed and 
used to predict the strain observed by the FOSGs. The predictions are compared with test results at quasi-static conditions.

\section{Magnetic Theory}

Developing a solid background of electromagnetic equations will both improve the qualitative understanding of the MB principles and supply the necessary tools for producing theoretical predictions for strain measurements. For references to these equations and extended derivations, refer to sources [35,36].

\section{Electrostatics}

Coulomb's law produces the force acting on a given charge due to surrounding charges and/or charge distributions. Equation (47) is Coulomb's law in its simplest form, the force on charge $Q$ exerted by charge $q$. The vector $\mathbf{d}$ is drawn from charge $Q$ to charge $q$. Note that throughout this derivation, the hat symbol denotes a unit vector. The relative permittivity $\varepsilon_{0}$ is $8.85 \times 10^{-12} \mathrm{C}^{2} / \mathrm{Nm}^{2}$ for force measured in Newtons, charge in Coulombs, and distance in meters.

$$
\mathbf{F}=\frac{1}{4 \pi \varepsilon_{0}} \frac{q Q}{d^{2}} \hat{\mathbf{d}}
$$

For a finite number of charges, the force acting on a given charge is given by Eq. (48).

$$
\mathbf{F}=\frac{Q}{4 \pi \varepsilon_{0}} \sum_{i} \frac{q_{i}}{d_{i}^{2}} \hat{\mathbf{d}}_{i}
$$

Introducing the concept of an electric field produces a vector field from which the force applied to an arbitrary charge at any location can be readily determined. The distance vector $\mathbf{d}_{i}$ is now drawn from the arbitrary location to the charge $q_{i}$. 


$$
\mathbf{E}=\frac{1}{4 \pi \varepsilon_{0}} \sum_{i} \frac{q_{i}}{d_{i}^{2}} \hat{\mathbf{d}}_{i}
$$

The electric field generated by a charge distribution is obtained by integration. The vector $\mathbf{r}$ designates the arbitrary location with respect to the origin. The charge density $\rho$ may vary throughout the integration volume.

$$
\mathbf{E}(\mathbf{r})=\frac{1}{4 \pi \varepsilon_{0}} \int \frac{\rho}{d^{2}} \hat{\mathbf{d}} d V
$$

With the intention of producing an equation similar to Eq. (50) in differential form, it is useful to consider the flux of the electric field. The flux through a closed surface of the electric field due to a single charge at the origin is show below.

$$
\oint_{s} \mathbf{E}(\mathbf{r}) \cdot d \mathbf{A}=\frac{q}{4 \pi \varepsilon_{0}} \int\left(\frac{1}{r^{2}} \hat{\mathbf{r}}\right) \cdot\left(\frac{r^{2} \sin \theta}{\cos \gamma} \hat{\mathbf{n}}\right) d \theta d \phi
$$

The angle $\gamma$ is the angle between the radius vector and the vector normal to the surface $\mathbf{n}$. This unusual way of representing the differential area is convenient because the cosine term cancels as a result of the dot product. The position squares cancel, resulting in and integral equal to $4 \pi$, and canceling yet another term from the equation. Since the shape of the closed surface is arbitrary, clearly this result can be applied to multiple charges or charge distributions enclosed by a surface. A similar procedure can prove that the electric field due to charges outside the closed surface produces zero net flux through the closed surface [37]. As a result, Eq. (52) applies in general to a charge distribution bound by closed surface $S$.

$$
\oint_{s} \mathbf{E}(\mathbf{r}) \cdot d \mathbf{A}=\int \frac{\rho}{\varepsilon_{0}} d V
$$


Applying the divergence theorem allows the area integral to be converted into a volume integral. Differentiating each side of the equation to remove the integrals produces Eq. (53), know as Gauss's law.

$$
\nabla \cdot \mathbf{E}=\frac{\rho}{\varepsilon_{0}}
$$

\section{Magnetostatics}

While electric fields apply a force to a charge dependent upon position, magnetic fields produce forces that are dependent on velocity. The force acting on a charge $Q$ moving with velocity $v$ is computed in Eq. (54), know as the Lorentz force law.

$$
\mathbf{F}=Q(\mathbf{v} \times \mathbf{B})
$$

In addition to being affected by magnetic fields, moving charges produce magnetic fields. Equation (55) is an approximation to the Biot-Savart law. This approximation illustrates the basic factors and properties of a magnetic field.

$$
\mathbf{B}=\frac{\mu_{0}}{4 \pi} \frac{q \mathbf{v} \times \hat{\mathbf{d}}}{d^{2}}
$$

The Biot-Savart law can be written in several forms. The volume integral form is displayed in Eq. (56). Here the charge and velocity terms from Eq. (55) are replaced by the current density (per unit volume) $\mathbf{J}$.

$$
\mathbf{B}(\mathbf{r})=\frac{\mu_{0}}{4 \pi} \int \frac{\mathbf{J} \times \hat{\mathbf{d}}}{d^{2}} d V
$$


By considering the divergence of Eq. (56) an important property of magnetic fields can be discovered. The right side of the equation can be shown to be zeros using a vector identity [36]. This demonstrates that the divergence of the magnetic filed is zero.

$$
\nabla \cdot \mathbf{B}=0
$$

If the curl is considered, the right side of Eq. (56) can be manipulated, again by a vector identity, to produce Ampere's law, Eq. (58).

$$
\nabla \times \mathbf{B}=\mu_{0} \mathbf{J}
$$

\section{Electrodynamics}

There remain two equations to complete the set of what is know as Maxwell's equations. The first concerns the effect of a changing magnetic field. Faraday's law of induction describes the electromotive force (EMF) E around a close loop caused by the change in magnetic flux through the loop.

$$
\mathbf{E}=-\frac{d \Phi}{d t}
$$

The EMF is also equal to the line integral of the electric field. Substituting the definition of magnetic flux and the line integral into Eq. (59) results in Eq. (60).

$$
\oint \mathbf{E} \cdot d \mathbf{l}=-\int \frac{\partial \mathbf{B}}{\partial t} \cdot d \mathbf{A}
$$

Using stokes theorem, the line integral can be convert into a volume integral. Differentiating each side produces Eq. (61), which is Faraday's law in differential form. 


$$
\nabla \times \mathbf{E}=-\frac{\partial \mathbf{B}}{\partial t}
$$

As mention previously, this law states that a changing magnetic field produces an electric field. As it turns out, a changing electric field also produces a magnetic field. Maxwell discovered that Ampere's law was incomplete. Taking the divergence of Eq. (58) produces the following equation.

$$
\nabla \cdot(\nabla \times \mathbf{B})=\nabla \cdot \mu_{0} \mathbf{J}
$$

The divergence of the curl of a vector field is zero, and therefore the left side of Eq. (62) zero. However, the right side is only zero for steady currents. Eq. (63) is the continuity equation for electric charge.

$$
\frac{\partial \rho}{\partial t}+\nabla \cdot \mathbf{J}=0
$$

Using the continuity equation and Gauss's law (Eq. (53)), the right side of Eq. (62) becomes a function of the change in electric field. Maxwell corrected Ampere's law by adding a term to the equation that causes a cancellation upon taking the divergence of the equation. Eq. (64) is Ampere's law with Maxwell's correction.

$$
\nabla \times \mathbf{B}=\mu_{0} \mathbf{J}+\mu_{0} \varepsilon_{0} \frac{\partial \mathbf{E}}{\partial t}
$$

\section{Magnetic Materials}

Dynamics of charges or currents in magnetic and electric fields acting in free space are readily computed using Maxwell's equations. While these equations apply in general, it is often useful to rewrite the equations for applications in matter. Rewriting the equations allows the externally applied electric and magnetic fields to be separated from those resulting from material properties. The analysis that follows emphasizes materials 
with significant magnetic properties. Many parallels can be drawn between magnetic and electrical material properties; however, the electrical properties will only be briefly mentioned.

Magnetic properties in materials are the result of magnetic dipoles that exist at the atomic level. In introductory texts, magnetic dipoles are often conceptualized as tiny magnets with north and south poles. In reality, dipoles are formed by electron currents within atoms. The motions of the electrons within atoms produces a magnetic field. Atoms with partially filled electron orbitals can have electrons motions that produce a nonzero average magnetic field. In a continuum, the average magnetic properties of the atoms become an acceptable representation for the magnetic properties of the continuum. In paramagnetic materials, an externally applied magnetic filed tends to align the magnetic dipoles thereby enhancing the magnetic field within the material. The electron motions can be represented as infinitesimal current loops. Eq. (54) can be rewritten to calculate the force applied to a current loop.

$$
\mathbf{F}=I \oint d \mathbf{l} \times \mathbf{B}
$$

For a uniform magnetic field, the components of the cross product cancel when integrated. Accordingly, the net force acting on a current loop in a uniform magnetic field is zero. Considering the contrapositive, if the net force on a current loop is nonzero, the magnetic field must not be uniform.

\section{Maxwell's Equations}

Equations (66) comprise what is know as Maxwell's equations. These include Gauss's law, Faraday's law, and Ampere's law with Maxwell's correction. 


$$
\begin{aligned}
& \nabla \cdot \mathbf{E}=\frac{\rho}{\varepsilon_{0}} \\
& \nabla \cdot \mathbf{B}=0 \\
& \nabla \times \mathbf{E}=-\frac{\partial \mathbf{B}}{\partial t} \\
& \nabla \times \mathbf{B}=\mu_{0} \mathbf{J}+\mu_{0} \varepsilon_{0} \frac{\partial \mathbf{E}}{\partial t}
\end{aligned}
$$

Summing the electric force from Eq. (50) and the magnetic force from Eq. (54) results in a force equation that, combined with Maxwell's laws, serves as a suitable foundation for examining stresses in magnetic materials.

$$
\mathbf{F}=Q(\mathbf{E}+\mathbf{v} \times \mathbf{B})
$$

Eq. (67) can be rewritten for force per unit volume.

$$
\mathbf{f}=\rho \mathbf{E}+\mathbf{J} \times \mathbf{B}
$$

Gauss's law and Ampere's law with Maxwell's correction can be substituted to obtain Eq. (69).

$$
\mathbf{f}=\varepsilon_{0}(\nabla \cdot \mathbf{E}) \mathbf{E}+\left(\frac{1}{\mu_{0}} \nabla \times \mathbf{B}-\varepsilon_{0} \frac{\partial \mathbf{E}}{\partial t}\right) \times \mathbf{B}
$$

Faraday's law can be applied to replace the time derivative of the electric field. This generates an additional term.

$$
\mathbf{f}=\varepsilon_{0}((\nabla \cdot \mathbf{E}) \mathbf{E}-\mathbf{E} \times(\nabla \times \mathbf{E}))-\left(\frac{1}{\mu_{0}} \mathbf{B} \times(\nabla \times \mathbf{B})\right)-\varepsilon_{0} \frac{\partial}{\partial t}(\mathbf{E} \times \mathbf{B})
$$


The terms involving two cross products can be replaced using a vector product rule. The result can be conveniently written using Maxwell's stress tensor T.

$$
\begin{aligned}
& \mathbf{f}=\nabla \cdot \mathbf{T}-\varepsilon_{0} \frac{\partial}{\partial t}(\mathbf{E} \times \mathbf{B}) \\
& T_{i j}=\varepsilon_{0}\left(E_{i} E_{j}-\frac{1}{2} \delta_{i j} E^{2}\right)+\frac{1}{\mu_{0}}\left(B_{i} B_{j}-\frac{1}{2} \delta_{i j} B^{2}\right)
\end{aligned}
$$

The net force acting on a body is obtained by integrating over the volume. The volume integral for the stress tensor can be converted into a surface integral using the divergence theorem. The first term is often used in FEA magnetics codes to compute the net magnetic force on object.

$$
\mathbf{F}=\oint_{S} \mathbf{T} \cdot d \mathbf{A}-\varepsilon_{0} \frac{d}{d t} \int(\mathbf{E} \times \mathbf{B}) d V
$$

\section{Elastic Theory}

Combining Maxwell's stress tensor with relevant equation from elastic theory produces a concise direct relation between the magnetic field and the strain. First, the definition of Maxwell's stress tensor can provide confusion as to the direction of the stress related its sign. Eq. (73) is Cauchy's equation of motion. The left side contains the acceleration terms, and the right side contains body force (per unit volume) and material stress terms respectively.

$$
\rho \mathbf{a}=\mathbf{f}+\nabla \cdot \widetilde{\mathbf{T}}
$$

By considering the static case and substituting the force from Eq. (71), the following relation is produced.

$$
\nabla \cdot \mathbf{T}=-\nabla \cdot \widetilde{\mathbf{T}}
$$


The stress tensor can now be employed to compute strain occurring in linear isotropic magnetic materials under static conditions. Eq. (75) calculates the strain matrix $\mathbf{S}$ from the stress tensor [38]. Young's modulus $E_{Y}$ and Poisson's ratio $v$ are material properties.

$$
S_{i j}=\frac{1}{E_{Y}}\left((1+v) \widetilde{T}_{i j}-v \widetilde{T}_{k k} \delta_{i j}\right)
$$

Inside a conductor, the electric field is zero and will not be considered further. Substituting the stress tensor into Eq. (75) produces the following equation.

$$
S_{i j}=-\frac{1}{\mu_{0} E_{Y}}\left((1+v) B_{i} B_{j}-\frac{1}{2} B^{2} \delta_{i j}\right)
$$

This result indicates that the resulting strain along the direction of the magnetic field will be compressive. The geometry of the MB causes the dominant component of the magnetic field at the FOSG to be radial. As a result, computing the strain from the magnetic field is relatively straightforward. However, the magnetic field must first be modeled for the MB.

\section{MB Magnetic Field Model}

Magnetic field models for electromechanical devices are typically developed using finite elements. In many instances, assumptions and simplifications allow the use of a twodimensional magnetic field models. Modelers are usually interested in obtaining the net force or torque generated as the primary function of the device. In these cases, reasonable predictions for the global magnetic field are required; however, accurate localized predictions are unnecessary. The force and torque values are compute in a fashion similar to Eq. (72), where the divergence theorem is utilized to compute the force with a surface integral. The result is that there are limited resources and available 
literature for high accuracy localized magnetic field predictions in electromechanical devices.

The task of modeling the magnetic field in an $\mathrm{MB}$ requires significant geometric complexity. The FOSGs are located at the axial end of the MB laminates. This require a three-dimensional analysis. The mesh density must also be great enough to achieve consistent results at the FOSG locations. The tight clearance of rotor and stator at the MB also requires high mesh density at the rotor-stator interface. An adequate FE model poses significant, but not insurmountable challenges to the modern day desktop computer. By reducing the model size with planes of symmetry, consistent results can be achieved with reasonable computation times.

The magnetic field of the MB is modeled using ANSYS, a commercial FE software package. The modeled geometry is displayed in Fig. 68. The model is simplified by slicing the model along the two planes of symmetry displayed in the figure. The rotor model is limited to extend $19 \mathrm{~mm}$ (.75 in) axially beyond the MB stator laminates. Further increase in the rotor length produces no effect on results, and increases computation time significantly. 

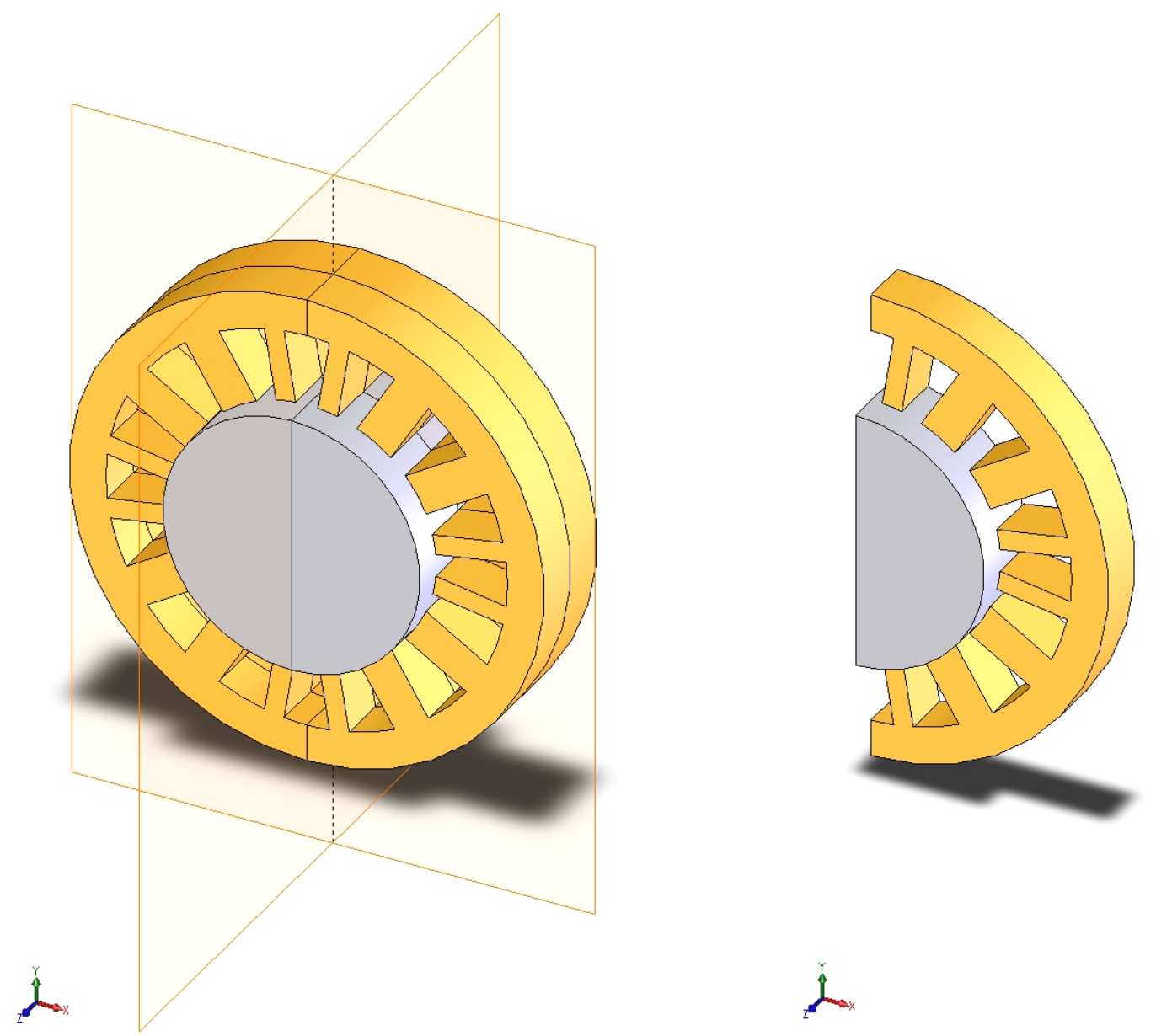

Fig. 68 MB FE model planes of symmetry

The mesh, displayed in Fig. 69, is generated automatically. Program assigned elements are used to model the three dimensional magnetic vector field. The mesh is refined at the FOSG locations and at the surface near the rotor-stator interface. The resulting FE model contains approximately 400,000 nodes and 300,000 elements.

Fig. 70 shows the model items representing the MB currents. The currents of each loop displayed in the figure are assigned at the beginning of each FE simulation. The geometry of the wire loops displayed on the right is an accurately representation of the wire coils in the MB. 


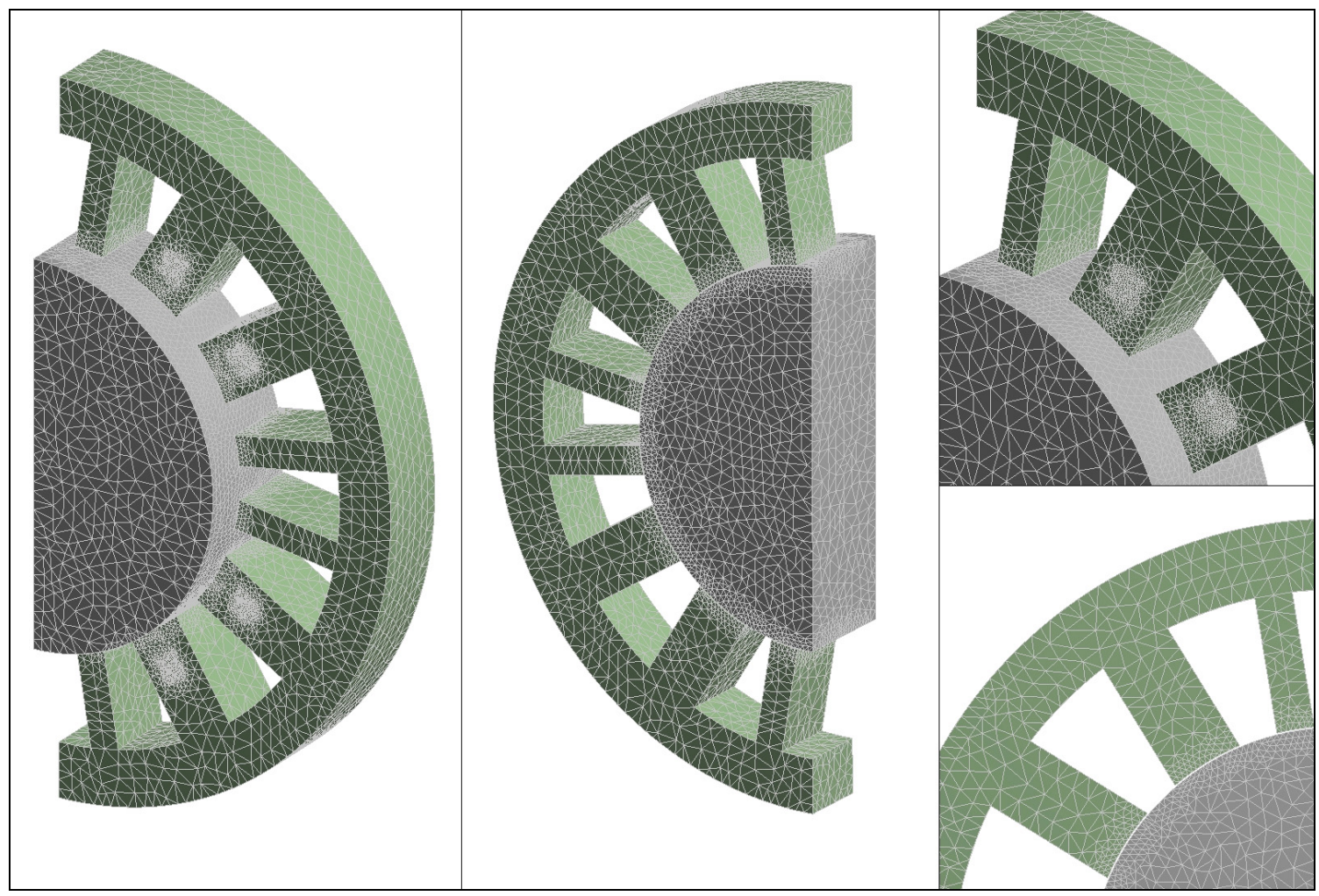

Fig. 69 MB FE model mesh
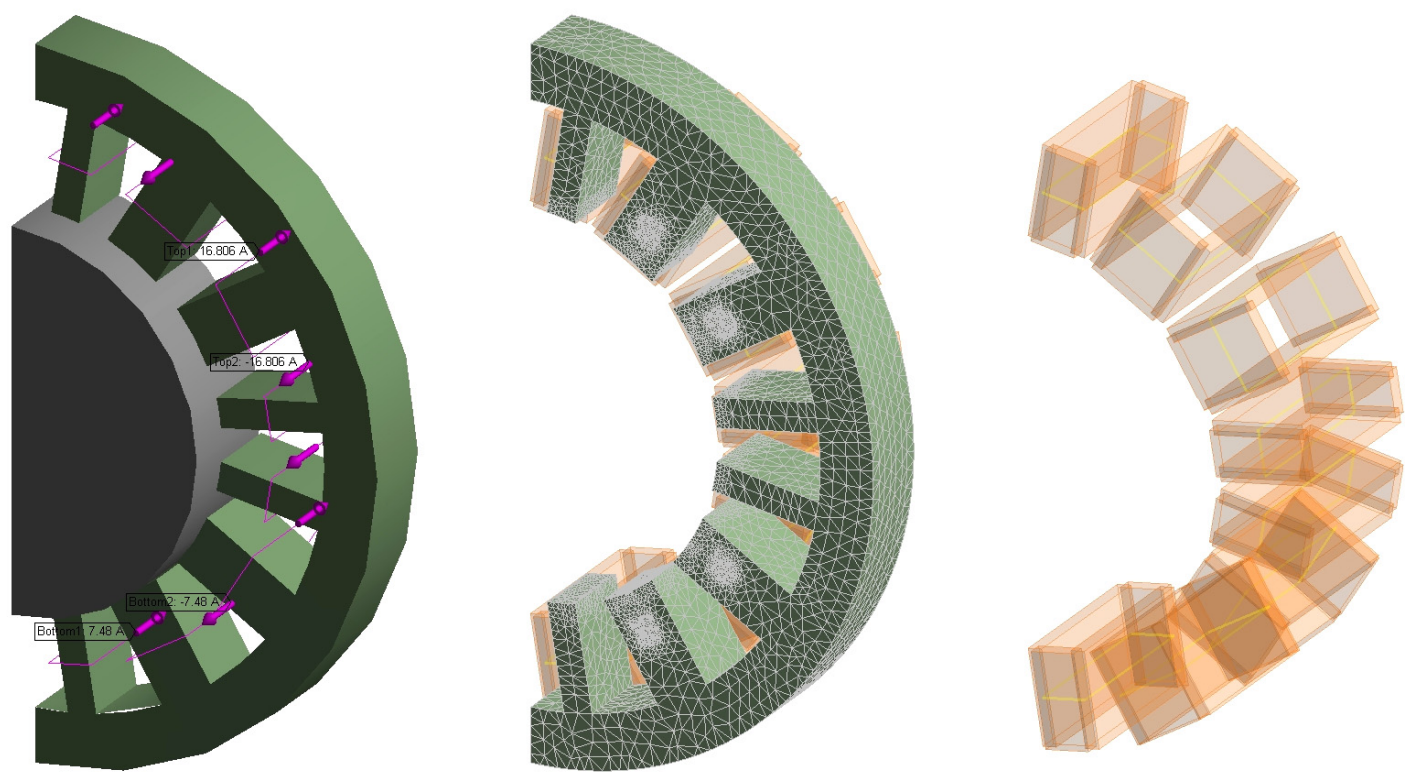

Fig. 70 MB FE model wires and currents 
The model also includes an enclosure that extends $13 \mathrm{~mm}(.5 \mathrm{in})$ in the radial direction beyond the MB stator. Parallel magnetic flux boundary conditions are applied the surfaces of the enclosure and the planes of symmetry. Fig. 71 shows the enclosure and the full mesh of the model.
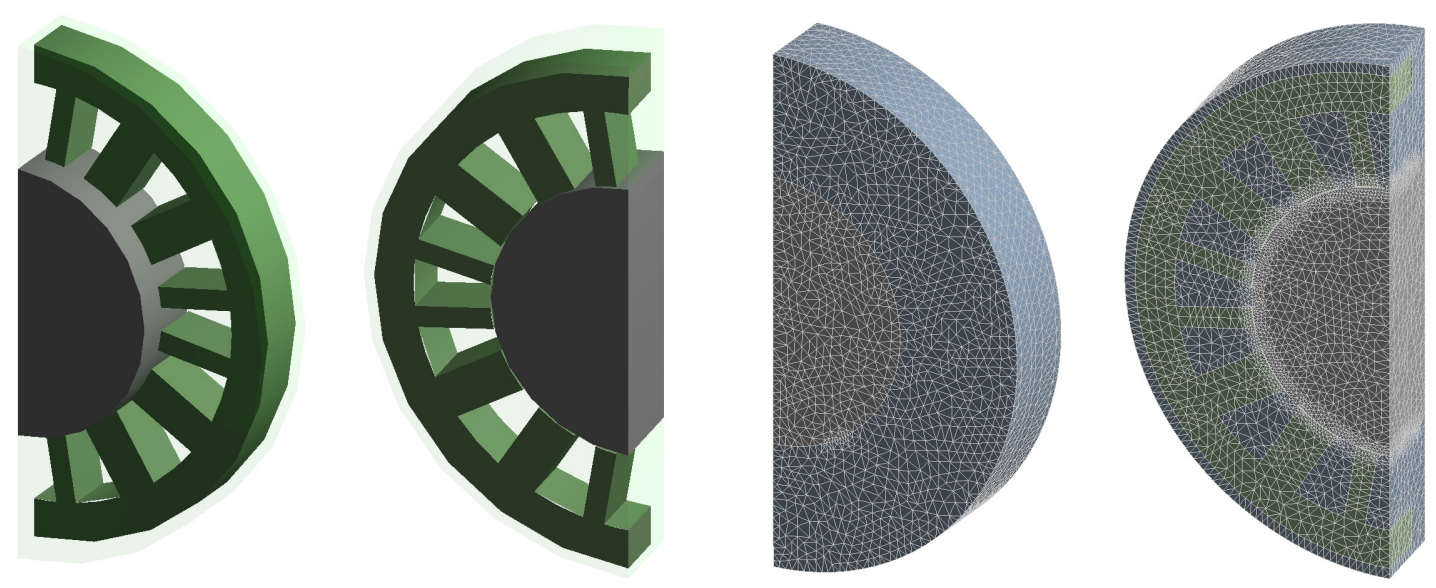

Fig. 71 MB FE model enclosure

The FOSG locations are displayed in Fig. 72. The magnetic field is determined at the locations and used to model the strain measurements. The strain predictions from each group of model points are averaged to determine an average strain prediction for each FOSG. 


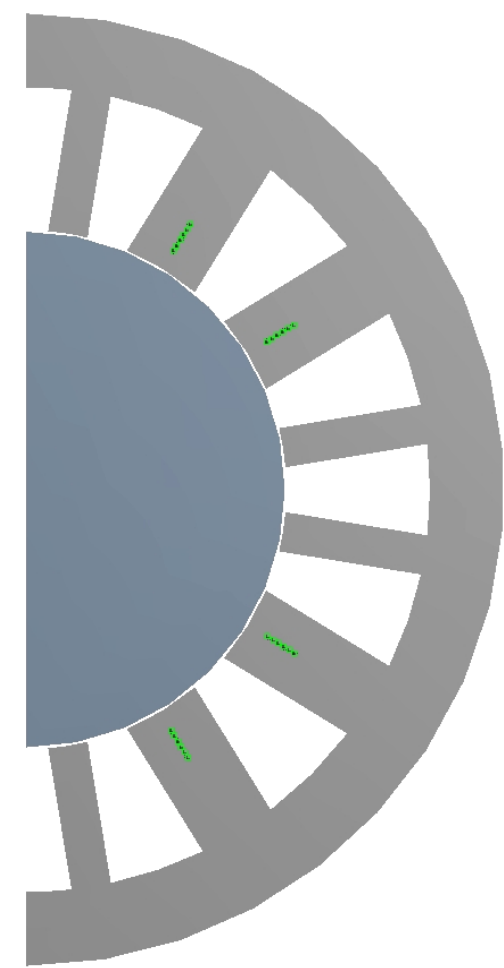

Fig. 72 MB FE model locations for FOSG measurements

The material properties of the $\mathrm{MB}$ rotor and stator models are provided by $\mathrm{SKF}$ Magnetic Bearings for M-19 steel. Fig. 73 shows the B-H curve for M-19 steel. The MB wires are modeled with the copper alloy model from the ANSYS material library. The enclosure material is air, also taken from the ANSYS material library. 


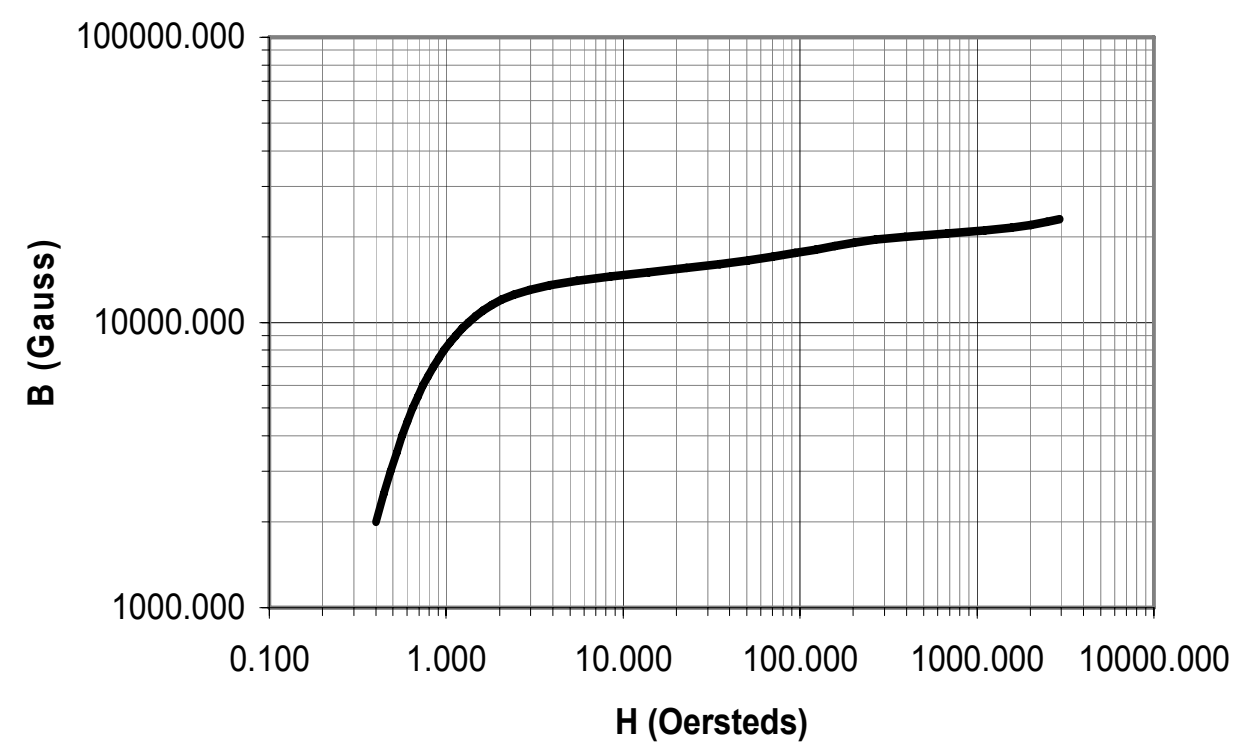

Fig. 73 B-H curve provide by SKF Magnetic Bearings, Inc.

\section{Results}

Experimental FOSG measurements are taken while the rotor is translated in the vertical direction. The vertical excitation preserves the symmetry assumed in the FE model. The excitation frequency is $3 \mathrm{~Hz}$ and the amplitude is $50 \mu \mathrm{m}$ (.002 in). Data are taken for many excitations and averaged to obtain measurements of rotor position, MB strain, and MB control currents. Ten data points are selected from the averaged period and used for the FE predictions. Fig. 74 and Fig. 75 show sample results for the FE magnetic field predictions at one of the data points. The magnetic field has the greatest intensity in the top poles, where the MB currents are the highest. Fig. 75 displays the magnetic vector field. 


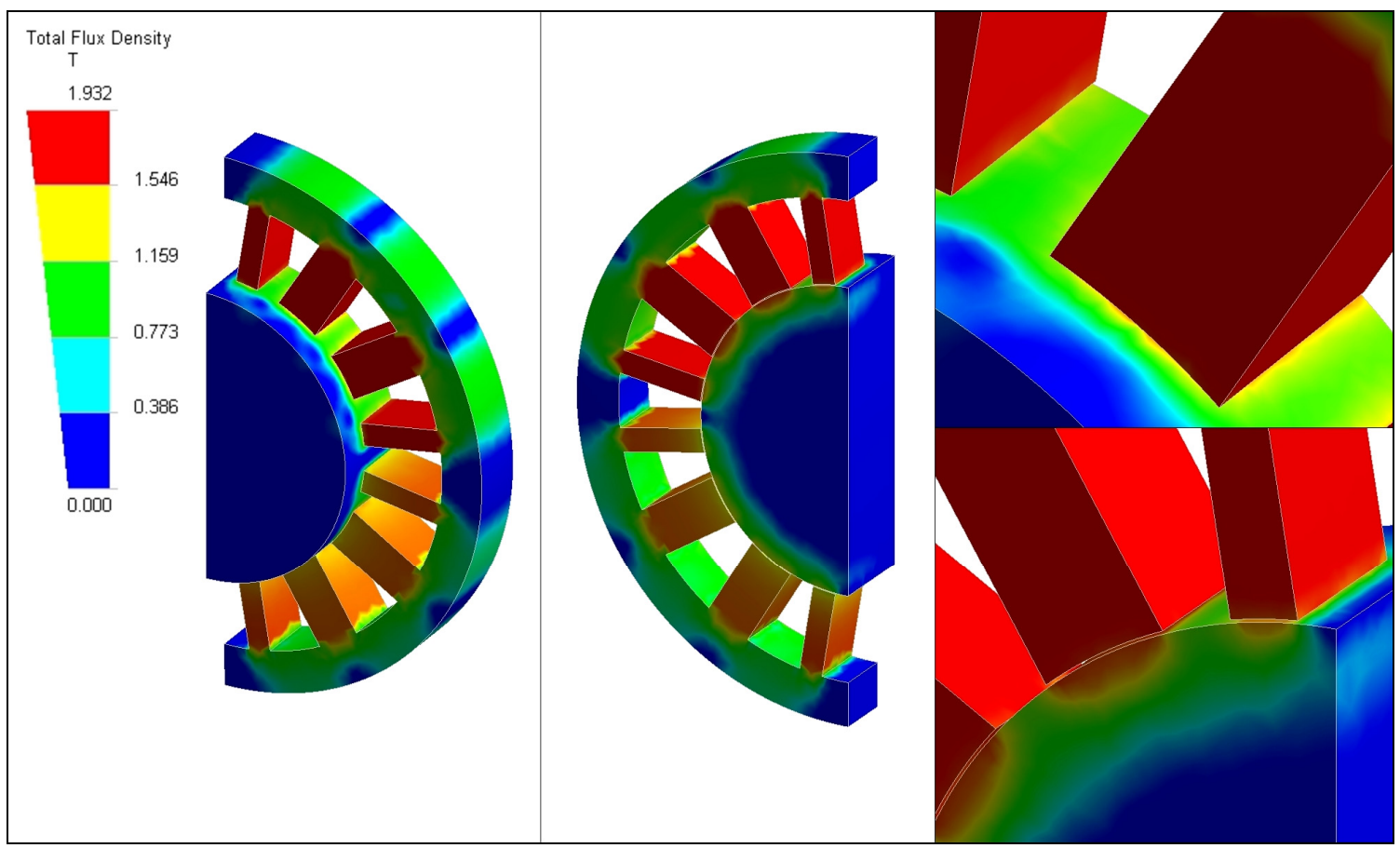

Fig. 74 MB FE model sample magnetic field

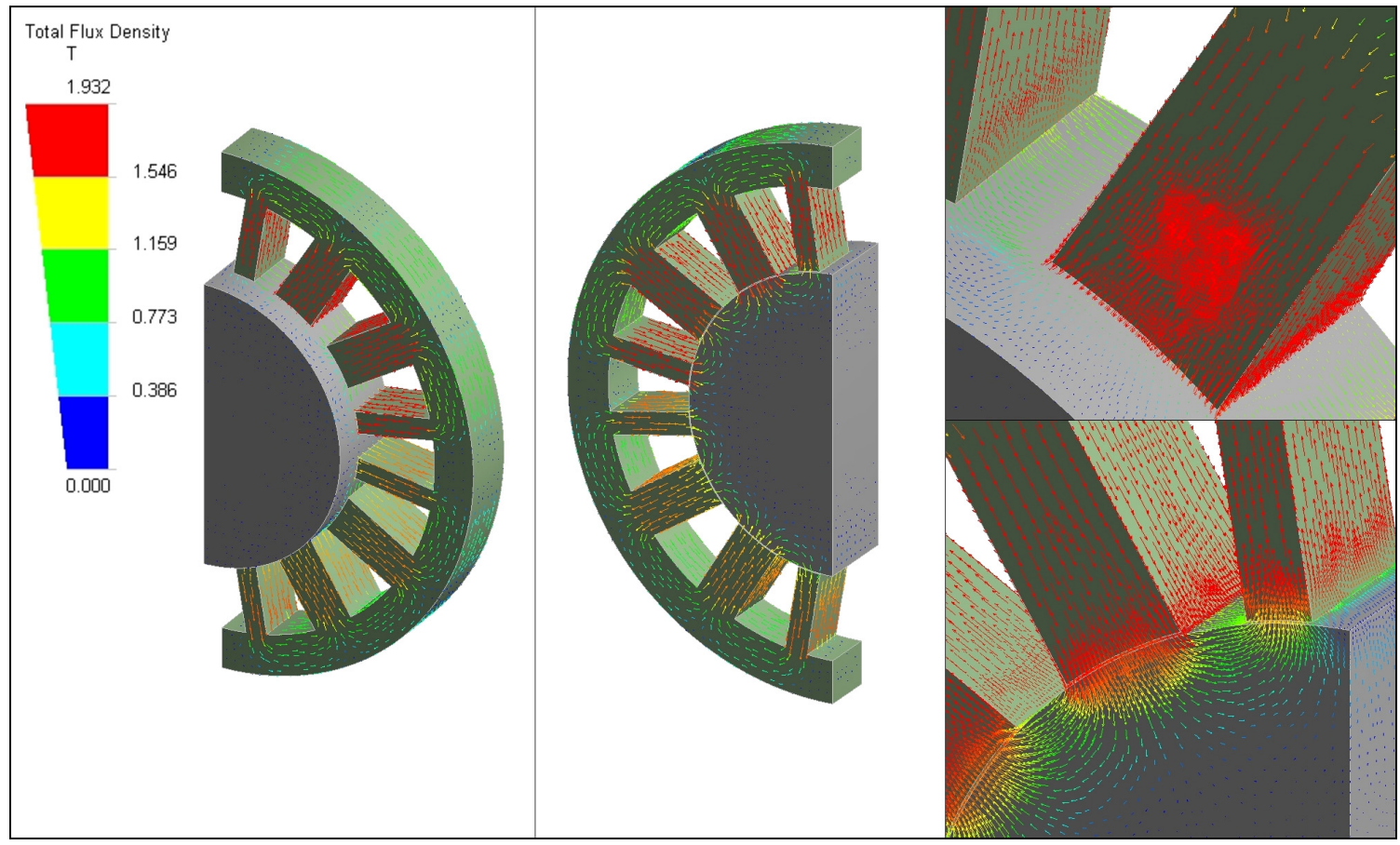

Fig. 75 MB FE model sample magnetic field vector plot 
The figures below compare experimental strain measurements and FE predictions. The non-drive bearing results (Fig. 76) display the same behavior in terms of relative strain amplitudes and phases. A true comparison of the experimental and predicted values (Fig. 77) demonstrates that the strain is typically over-predicted.
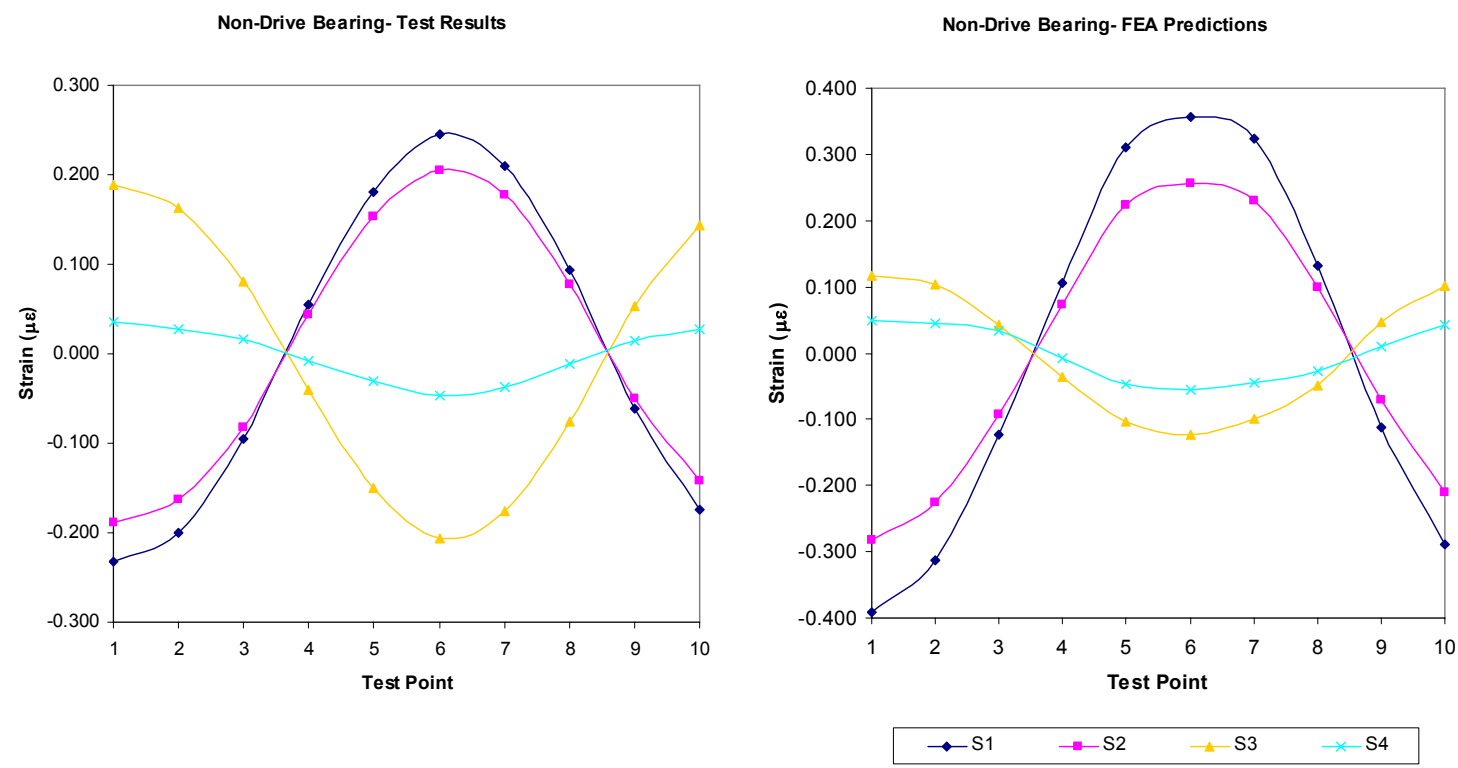

Fig. 76 Non-drive bearing strain test results and FE predictions 

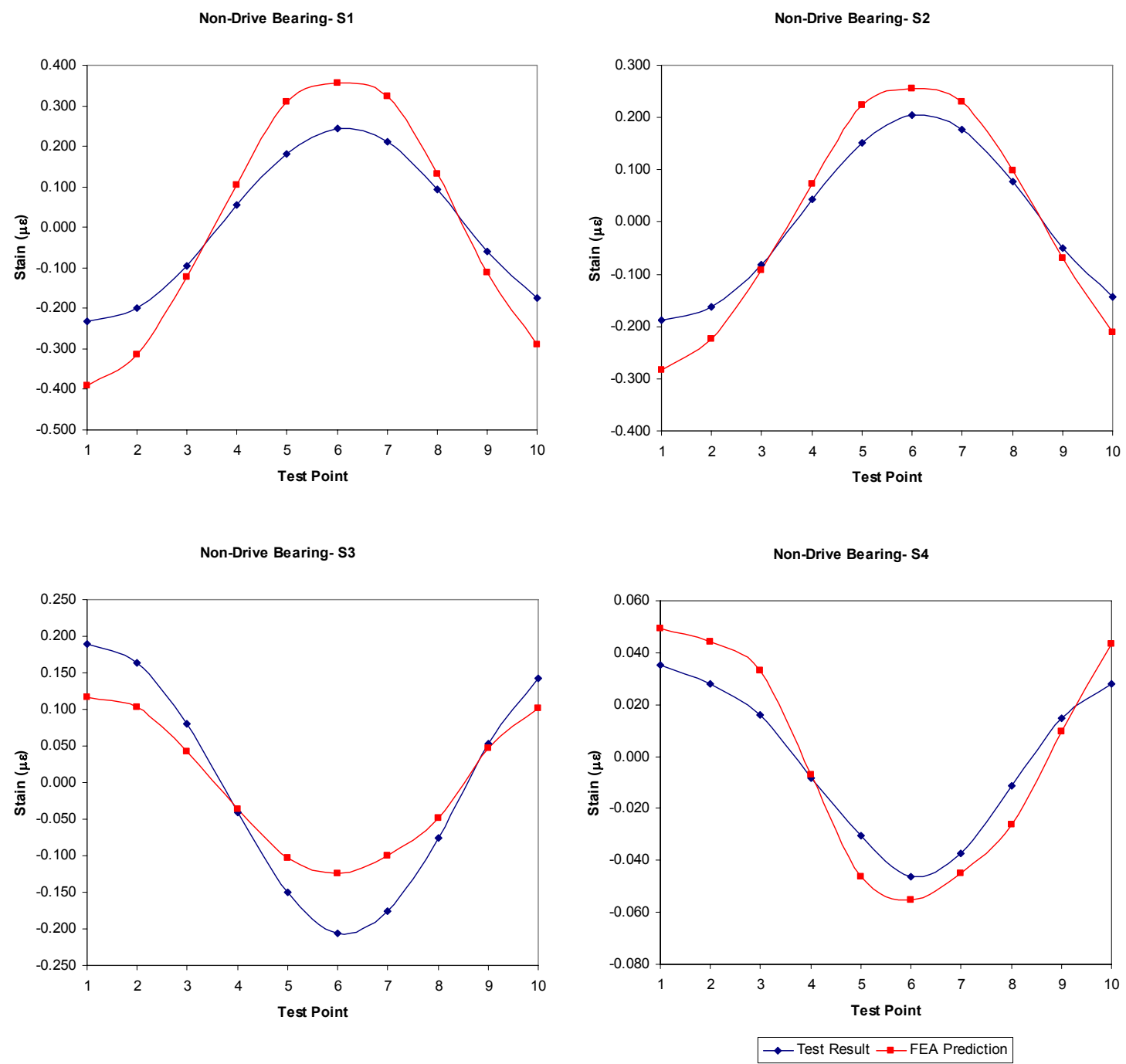

Fig. 77 Non-drive bearing comparison of test results and predictions 
The drive bearing predictions also match the behavior of the experimental results qualitatively (Fig. 78). The direct comparison (Fig. 79) of the drive bearing results contains noticeable discrepancies. The predictions for FOGSs S2 and S3 contain irregularities that can be attributed to their small amplitudes. Predictions for FOSG S4 are much larger than experimental results, suggesting that this sensor is either malfunctioning or is poorly bonded to the MB laminates. In general, the inaccuracies of the drive bearing results may also be attributed to excessive rotor-stator clearance resulting from remachining after a high-speed delevitaion.
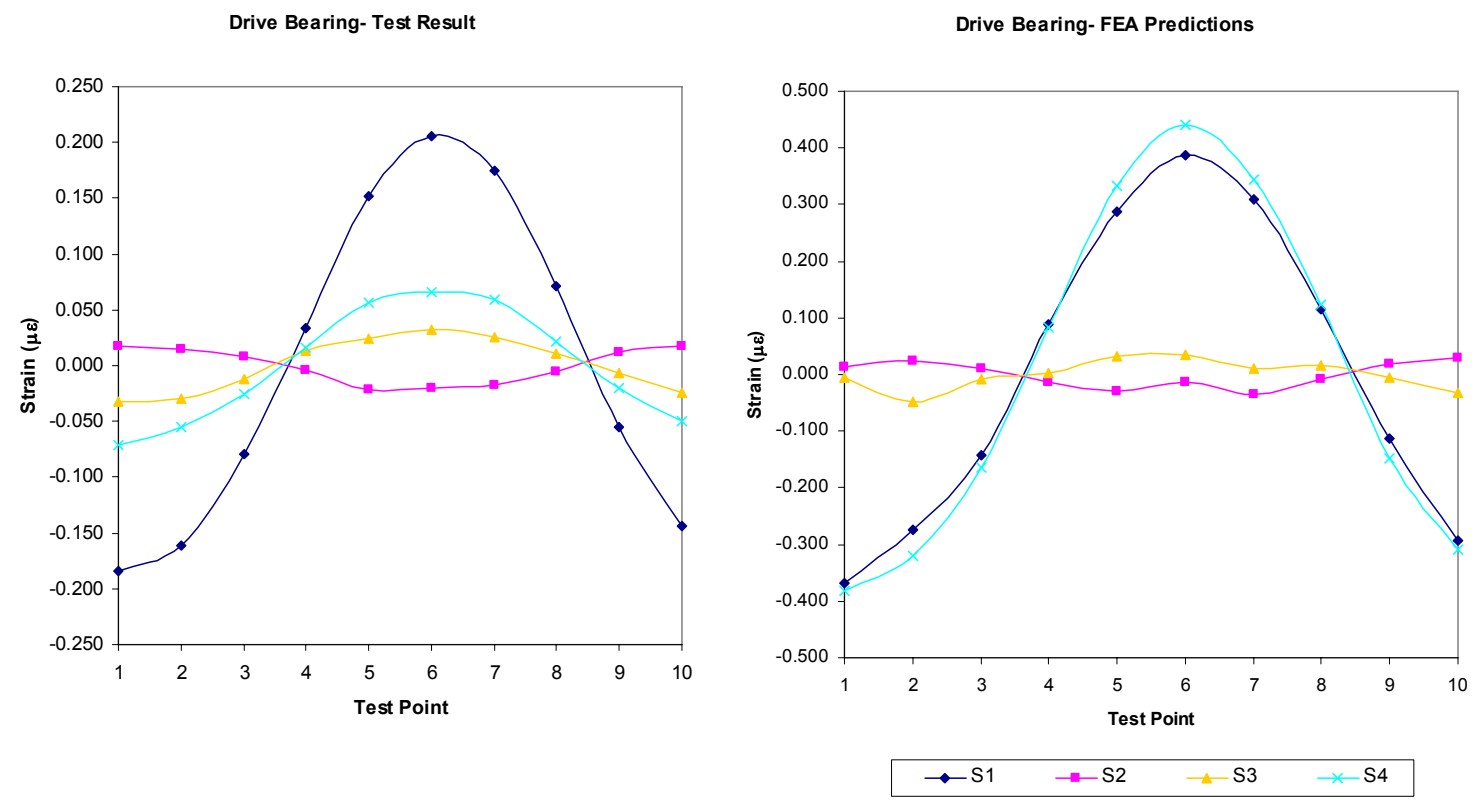

Fig. 78 Drive bearing strain test results and FE predictions 

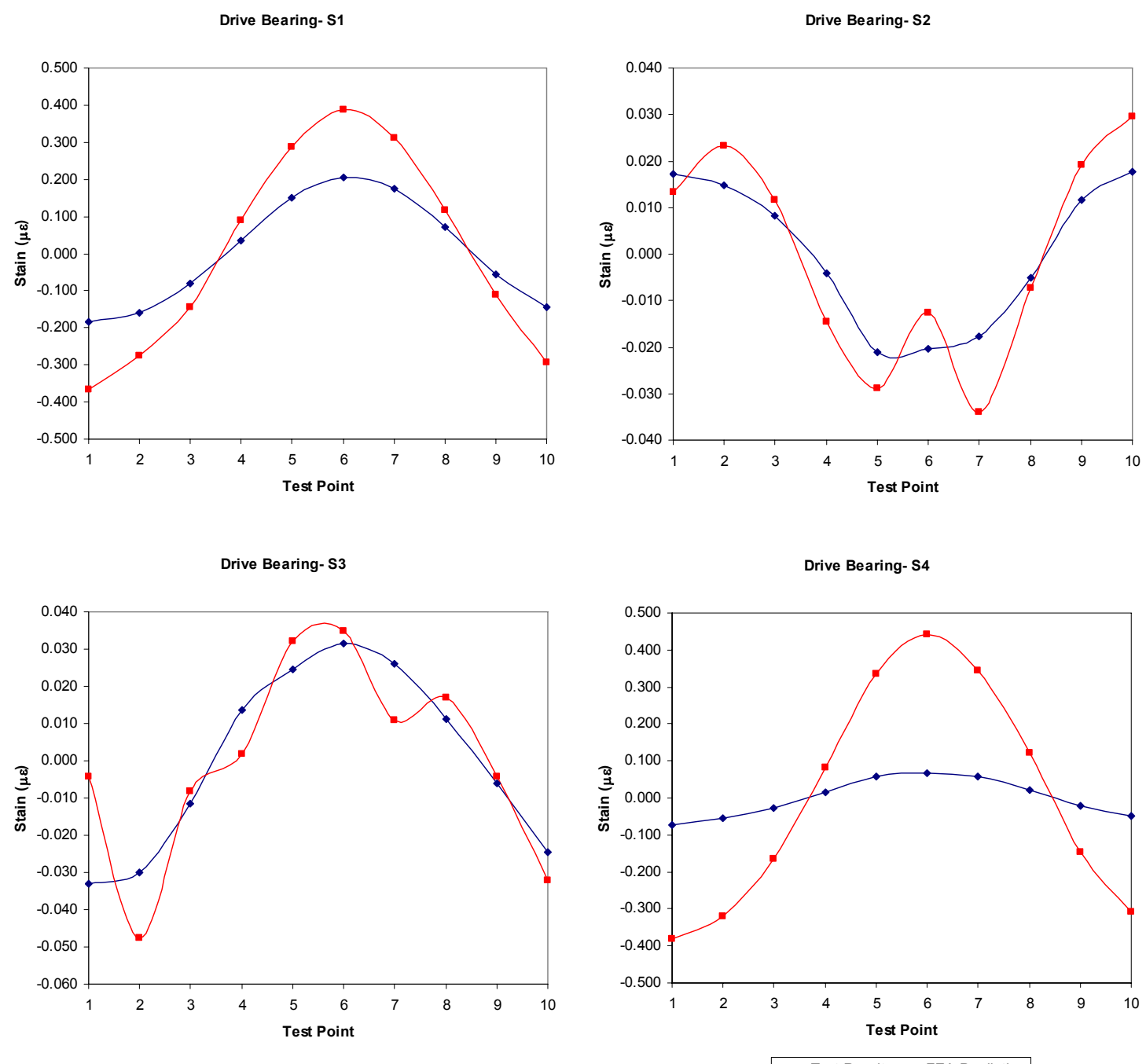

Fig. 79 Non-drive bearing comparison of test results and predictions

\section{Magnetostriction Summary}

FE predictions correlate reasonably well with experimental results. The FE predictions capture the correct phase and relative amplitudes of experimental results. A direct comparison of the results shows reasonable agreement in most case. This comparison also reveals noticeable discrepancies in several cases. 
There are a number of factors that can contribute to the discrepancies. The model mesh size was limited by the computational capabilities and available run time. Further mesh refinement might smooth some of the irregular predictions. The FE model does not include the bond interface between the FOSG and the MB laminates, which may contribute to the over-prediction of the strain. The FE model uses ideal MB and coil geometries and material properties. Any damage or manufacturing error in the rotor, stator, or wire coils, or local variation in material properties could alter the behavior of the MB. An example of such a defect is the delamination that has occurred on the MB stator. 


\section{CONCLUSIONS AND EXTENSIONS}

The section summarizes the theory, experimental process, results, and conclusions detailed previously. The contributions of the research to the present body of knowledge are considered. The author addresses the applications and extensions including recommendations for future testing.

\section{Research Summary}

This research effort focuses on identifying air seal coefficients on a flexible rotor system using MBs. This research aim is to develop a test method applicable for identification of rotordynamic forces in a real turbomachine. One primary application of this method is to identify impeller coefficients in high-performance, shrouded, centrifugal compressors. The present research provides a significant step toward accomplishing this objective by producing an identification method capable of measuring impeller coefficients.

The identification developments are made possible by the use of MBs as calibrated exciters. MBs have long been recognized for their potential to simultaneous support a rotor and gather test data for experimentation. Historical attempts to use MBs as calibrated exciters have experienced limited success because of low force measurement accuracy. In this research, FOSGs have been installed in MBs and used for a variety of rotordynamic testing. In contrast to other attempts, FOSGs have produced low noise, high sensitivity force measurements; uncertainties are an order of magnitude less than other methods discussed in literature.

Air seals have been added to the MB test rig to provide rotordynamic forces for identification. Air seals with a supply pressure of $1.83 \mathrm{MPa}$ (265 psia) are installed at the midspan of the rotor. The seals produce a noticeable effect upon the system, in particular at the first bending mode. The air seal forces are modeled as direct and crosscoupled stiffness terms and direct damping terms. The system changes due to the 
addition of the air seals are readily observed by considering the system transfer functions from $\mathrm{MB}$ force to MB position measurements known as DFTFs.

The first step in determining experimental DFTFs or other system characteristics is the calibration of the FOSGs. The FOSGs are calibrated by exciting the system with horizontal and vertical translations of the rotor at frequencies well below the first bending mode. The inertial force of the rotor is computed using experimental rotor position measurements and MB housing acceleration measurements base on an FE rotor model. Gaussian least squares differential correction method is then used to determine the FOSG calibrations that produce the best fit of computed inertial force.

The FE model used in previous research and for the FOSG calibration is further developed for use in the identification process. The FE model is tuned to match freefree rotor characteristics without the seals in operation. The rotor FE model is then treated as known and used to identify the seal coefficients at the following four test conditions: (i) $0 \mathrm{rpm}$, no preswirl, (ii) $7700 \mathrm{rpm}$, no preswirl, (iii) $0 \mathrm{rpm}$ with preswirl, (iv) $7700 \mathrm{rpm}$ with preswirl.

Frequency domain identification is accomplished by minimizing the experiment versus model error of the rotor-seal DFTFs. Initial values for the seal coefficients are assumed, and the values are iterated using a general non-derivative search algorithm to match the modeled DFTFs to the experimental DFTFs. The results exhibit consistent seal coefficients for repeated tests at the same operating conditions. Uncertainty analysis suggests that the identified coefficient have a high degree of precision and that changes in operating conditions may account the small variation in results from test to test.

Time domain identification methods encounter a variety of problems that prevent any successful determination of seal coefficients. The direct integration of the model equations is precluded by the existence of unstable modes for the identification system. 
An advanced time domain approach known as Observer/Kalman Filter Identification is can identify a system with unstable modes, but it encounters numerical difficulties associated with the large frequency separation between rigid and flexible rotor modes. In addition, test rig limitations prevent accurate force measurements at low frequencies, and the imbalance response of the rotor introduces cyclic errors to the identification process.

The final method for determining seal coefficients is static measurement of the stiffness coefficients. The stiffness coefficients are measured using experimental measurements of the rotor position and MB currents. The cross-coupled stiffness coefficients are determined, but with noticeably larger uncertainties than in the frequency domain identification. Manufacturer specified uncertainties for force measurements from a current-position model assume zeros eccentricity. The rotor must be displaced from the centered position to measure the stiffness coefficients, and uncertainties are substantially larger than the specifications as a result. Direct stiffness measurements are of the same magnitude as the measurement uncertainties.

Frequency domain identification produces superior results because of several advantages over the other methods. The frequency domain identification uses an FFT of the raw time data prior to the identification process. This prevents noise at non-test frequencies and imbalance response from negatively impacting results. The frequency domain identification focuses on a narrow frequency band that is intentionally selected because the system exhibits a dramatic change in response due to the presence of the air seal. Frequencies are avoided that have force measurement limitations. Finally, frequency domain identification uses DFTFs that exaggerate the seal forces. The static measurements attempt to directly measure the seal forces, while the frequency domain method focuses on the characteristics of the first bending mode, which are extremely sensitive to the seal forces. The results from the present research on the MB test rig 
demonstrate that frequency domain identification is better suited for parameter identification in this situation.

The secondary focus of the research has been to better understand the mechanism causing strain in MBs. This topic has little background in the literature primarily because strain levels are insignificant from an MB design standpoint. Derivations based on common equations from the fields of electricity, magnetism, and continuum mechanics show that strain in a paramagnetic material caused by a magnetic field, a property known as magnetostriction, can be directly computed if the magnetic field is known.

FEA of the MBs is conducted to model the magnetic fields. A commercial software package, ANSYS, is used to develop a 3-dimensional model incorporating a non-linear magnetization curve. The model is simplified by utilizing planes of symmetry, and the magnetic field in the proximity of each FOSG is computed for 10 data points. The magnetic field predictions are used to compute strain, and the predictions are compared with experimental results. The phases and relative amplitudes of the predictions correlate well with experimental results. In general, strain is over-predicted, and in cases involving low strain levels, some erratic predictions occur. One FOSG appears to have a bonding complication based on the low experimental strain levels observed.

The MB magnetic FEA offers an insight into the force measurements used in the identification process. Both endeavors, the identification and the magnetic FEA, have yielded results that are reasonable, useful, and in some cases promising. Some of the methods developed herein can readily be applied to impeller or seal coefficient testing. This research serves as and adequate foundation for future rotordynamic testing in real turbomachines. 


\section{Extensions}

Impeller coefficient testing has been cited as a primary motivation for the present research, and there are significant commonalities worthy of mention. Impeller forces likely produce a significant changes in the system response versus the free rotor behavior. In particular, destabilizing impeller forces, potentially the cause of many historic cases of compressor instability, will be readily observable from DFTFs. The availability of compressors with MBs provides not only the ability to use the MBs for testing, but also a reduction in the system complexity from an identification standpoint. As demonstrated previously, the MBs are not part of the identification system model; the model therefore includes only the rotor, the impeller forces, and any seal forces.

However, there are some noticeable differences between impeller testing and the present research. The present research did not identify moment coefficients, which would likely be present in impeller testing. This issue could be addressed by using test frequencies around the first and second bending modes. With the proper rotor configuration, the first mode would primarily determine the lateral coefficients, and the second mode would determine the moment coefficients. Another complication is that the impeller identification system includes several seals. These seals would require adequate models or separate testing so that impeller forces can be extracted from the test results.

There are also several recommendations to consider. The air seal coefficients corresponding to larger force levels are more accurately identified. Accordingly, the operating conditions should be selected to maximize the impeller forces subject to the constraint of remaining applicable for typical field configurations. Benefits will likely be realized by using new high quality MBs. The MB test rig at the Turbomahinery Laboratory, while suitable for the present and similar test programs, exhibits some effects from aging and excessive MB clearance. Finally, more FOSG measurements at different poles within the MBs would not only provide redundancy in case a FOSG were 
damaged, but it would likely improve the force measurements by providing a better characterization of the MB behavior.

A potential test program for the MB test rig at the Turbomachinery Laboratory involves determining coefficients of frequency dependent seals. The recommended approach is to determine a set of basis functions for the seal coefficients, and identify constants for the basis functions. If this is not possible, or if it precludes the scientific process of the intended research, literature by San Andres [39], De Santiago [40], and Balantrapu [41] addresses parameter identification at each test frequency. Post-identification coefficient trending could then be performed to alleviate larger uncertainties associate with calculating coefficients separately at each frequency.

The successful use of MBs as calibrated exciters for air seal testing opens the door for future test programs. Just as this test program has relied on those before it, perhaps this effort will contribute to the future understanding of untested rotordynamic phenomena. The author would like to express his continued interest in the development of this line of research, and he would enjoy offering any advice or insight, albeit limited as an initial condition and decreasing with time, to interested parties. In the words of John Donne, "No man in an island, entire of itself." 


\section{REFERENCES}

[1] SKF Magnetic Bearings, 2006, "How They Work," http://www.revolve.com

[2] Fiber Dynamics Inc., 2003, "Products," http://www.fiberdynamics.com

[3] Childs D., 1993, Turbomachinery Rotordynamics: Phenomena, Modeling, \& Analysis, John Wiley \& Sons Inc., New York.

[4] Vance J., 1988, Rotordynamics of Turbomachinery, John Wiley \& Sons Inc., New York.

[5] Crassidis J., and Junkins J., 2004, Optimal Estimation of Dynamic Systems, CRC Press LLC, New York.

[6] Juang J., 1994, Applied System Identification, Prentice Hall PTR, Englewood Cliffs, New Jersey.

[7] Ljung L., 1999, System Identification, Prentice Hall PTR, Upper Saddle River, New Jersey.

[8] Zutavern Z., 2004, "Fiber Optic Strain Gauge Calibration and Dynamic Flexibility Transfer Function Identification in Magnetic Bearings," M.S. thesis, Texas A\&M University, College Station, Texas.

[9] Traxler A., and Schweitzer G., 1984, Measurement of the Force Characteristics of a Contactless Electromagnetic Rotor Bearing, Institute for Mechanics, ETH Zurich, Switzerland. 
[10] Lee C., Ha Y., and Kim C., 1994, "Identification of Active Magnetic Bearing System Using Magnetic Force Measurement," Proc. $4^{\text {th }}$ International Symposium on Magnetic Bearings, Zurich, pp 305-309.

[11] Matros M., Sobotzik J., and Nordmann R., 1996, “A New Model-Based Method for the Accurate Measurement of Magnetic Bearing Forces," Proc. $4^{\text {th }}$ International Symposium on Magnetic Suspension Technology, Gifu City, Japan, pp 239-248.

[12] Fittro R., Baun D., Maslen E., and Allaire P., 1997, "Calibration of an 8-Pole Planar Radial Magnetic Actuator,” ASME Paper 97-GT-18, Proc. International Gas Turbine \& Aeroengine Congress \& Exhibition, Orlando, Florida.

[13] Gahler C., 1998, "Rotor Dynamic Testing and Control with Active Magnetic Bearings,” Ph.D. dissertation, ETH, Zurich, Switzerland.

[14] Knopf E., and Nordmann R., 2000, "Identification of the Dynamic Characteristics of Turbulent Journal Bearings Using Active Magnetic Bearings," IMechE Paper, C576/110/2000.

[15] Pottie K., Matthijssen J., Norbart C., and Gielen L., 1999, "Modal Parameter Estimation of Rotation Machinery," IMechE, C556/005/99.

[16] Raymer S., and Childs D., 2001, "Force Measurements in Magnetic Bearings Using Fiber Optic Strain Gauges," ASME Paper 2001-GT-0027, Proc. International Gas Turbine \& Aeroengine Congress \& Exhibition, New Orleans, Louisiana. 
[17] Pavesi L., 2002, "Force Measurement in Magnetic Bearings Using Fiber Optic Strain Gauges,” M.S. Thesis, Texas A\&M University, College Station, Texas.

[18] Zutavern Z., and Childs D., 2005, "Fiber-Optic Strain Gauge Calibration and Dynamic Flexibility Transfer Function Identification in Magnetic Bearings," Proc. ASME TurboExpo 2005, GT2005-68484.

[19] Product Literature, 2000, Fiberdynamics Inc. Bryan, Texas.

[20] Raymer S., 2000, "Force Measurements in Magnetic Bearings Using Fiber Optic Strain Gauges,” M.S. thesis, Texas A\&M University, College Station, Texas.

[21] Picardo A., 2003, "High Pressure Testing of See-through Labyrinth Seals," M.S. thesis, Texas A\&M University, College Station, Texas.

[22] Wade J., 2004, “Test versus Predictions for Rotordynamic Coefficients and Leakage Rates of Hole-Pattern Gas Seals at Two Clearances in Choked and Unchoked Conditions," M.S. thesis, Texas A\&M University, College Station, Texas.

[23] Seifert B. 2005, "Measurements versus Predictions for Rotordynamic Coefficients and Leakage Rates for a Novel Hole-Pattern Seal," M.S. thesis, Texas A\&M University, College Station, Texas.

[24] Ertas B., 2005, "Rotordynamic Force Coefficients of Pocket Damper Seals," Ph.D. dissertation, Texas A\&M University, College Station, Texas. 
[25] Kerr B., 2005, "Experimental and Theoretical Rotordynamic Coefficients and Leakage of Straight Smooth Annular Gas Seals," M.S. thesis, Texas A\&M University, College Station, Texas.

[26] Nelson C. C., Childs D. W., Nicks C., and Elrod D., 1986, "Theory versus Experiment for the Rotordynamic Coefficients of Annular Gas Seals: Part 2 Constant-Clearance and Convergent Tapered Geometry," ASME Journal of Tribology, 108, pp. 433-438.

[27] Nelson H., and McVaugh J., 1976, "The Dynamics of Rotor-Bearing Systems Using Finite Elements,” Journal of Engineering for Industry, 98, pp. 593-600.

[28] Maslen E., Vazquez J., and Sortore C., 2002, "Reconciliation of Rotordynamic Models with Experimental Data," Journal of Engineering for Gas Turbines and Power, 124, pp. 351-356.

[29] Wang Q., and Maslen E., 2005, "Identification of Frequency Dependent Parameters in a Flexible Rotor System," Proc. ASME Turbo Expo 2005, GT2005-68577.

[30] Wang Q., Maslen E., and Ahn H., 2003, "Incorporating Data Uncertainty in Rotordynamic Model Reconciliation,” Proc. AMSE Turbo Expo 2003, GT200338595.

[31] Lagarias J., Reeds J., Wright M., and Wright P., 1998, "Convergence Properties of the Nelder-Mead Simplex Method in Low Dimensions," SIAM Journal of Optimization, 9(1), pp. 112-147. 
[32] Moler C., and Loan C., 2003, "Nineteen Dubious Ways to Compute the Exponential of a Matrix, Twenty-Five Years Later," Society for Industrial and Applied Mathematics Review, 45(1), pp. 3-49.

[33] Phan M., Horta L., Juang J., and Longman R., 1992, "Linear System Identification Via an Asymptotically Stable Observer," NASA Technical Paper, 3164 .

[34] Juang J., and Pappa R., 1985, “An Eigensystem Realization Algorithm for Modal Parameter Identification and Model Reduction," Journal of Guidance, Control, and Dynamics, 8(5), pp. 620-627.

[35] Young H. D., and Freedman R. A., 1996, University Physics, Addison-Wesley Publishing Company, Inc., Reading, Massachusetts.

[36] Griffiths D. J., 1999 Introduction to Electrodynamics, Prentice Hall, Upper Saddle River, New Jersey.

[37] Geraint W., and Rosser V., 1997, Interpretation of Classical Electromagnetism, Kluwer Academic Publishers, Dordrecht.

[38] Lai W. M., Rubin D., and Krempl E., 1993, Introduction to Continuum Mechanics. Butterworth Heinemann, Woburn, Massachusetts.

[39] San Andres L., 2003, "A Method for Identification of Foce Coefficients in Flexible Rotor-Bearing Systems," Technical Report to the Turbomachinery Research Consortium, Texas A\&M University, College Station, Texas. 
[40] De Santiago O., 2002, “Identification of Bearing Supports' Force Coefficients from Rotor Response Due to Imablances and Impact Loads," Ph.D. dissertation, Texas A\&M University, College Station, Texas.

[41] Balantrapu K., and San Andres L., 2004, "Identification of Force Coefficients in Flexible Rotor-Bearing Systems- Enhancements and Further Validations," Technical Report to the Turbomachinery Research Consortium, Texas A\&M University, College Station, Texas. 


\section{APPENDIX A SEAL SELECTION AND DESIGN}

\section{Seal Selection: Air Seal vs. Water Seal}

To reiterate, the overall purpose of the magnetic bearing test rig is parameter identification in real turbomachines. A major step toward this end is performing parameter identification on the current test rig. A modification to add back-to-back seals at the axial center of the rotor could produce suitable forces for identification. The first step in the design process is to consider what type of seal, water or air, is most suitable for achieving this objective. In both cases, smooth seals are considered because of the availability of theoretical predictions and test data for comparison.

\section{Air Seal Analysis}

The first modification to be considered for the magnetic bearing test rig is the addition of smooth back-to-back air seals. The primary issues are flow rate requirements and seal force levels as compared with the uncertainty of previous measurements on the test rig. An air seal coefficient prediction code developed at the Turbomachinery Laboratory, $X L I s o t S l$, is used to analyze the air seals.

Table 13 displays the parameters used in the seal analysis. Default values are used for air properties, loss coefficients, and the exit recovery factor. The volume to area ratio is set to zero for a smooth seal analysis. The seal diameter is the current rotor diameter. A seal length of 4 inches is selected. The radial clearances that are considered are (with zero taper) $250,380,510,760,1020 \mu \mathrm{m}(10,15,20,30$, and 40 mils $)$. The inlet pressure is set to $1.83 \mathrm{MPa}$ (265 psia). The back pressure was also varied from .758 $\mathrm{MPa}$ to 1.83 $\mathrm{MPa}$ (110 to 250 psia). A minimum back pressure of $.758 \mathrm{MPa}$ (110 psia) was used because further reduction in back pressure resulted in XLIsotSl code errors for large clearances. It is later shown that further reduction in the back pressure does not significantly affect the conclusions of this analysis. 
Table 13 Parameters for XlsotSl

\begin{tabular}{|c|c|c|}
\hline Reservoir Temperature & 80 & Deg F \\
\hline Seal Diameter & 3.917 & \\
\hline Seal Length & 4 & inches \\
\hline Inlet Clearance & 0.02 & i \\
\hline Exit Clearance & 0.02 & inches \\
\hline Cell Vol to Area Ratio & 0 & iches \\
\hline Inlet Preswirl Ratio & 0 & -- \\
\hline Entrance Loss Coefficient & 0 & -- \\
\hline Exit Recovery Factor & 1 & \\
\hline
\end{tabular}

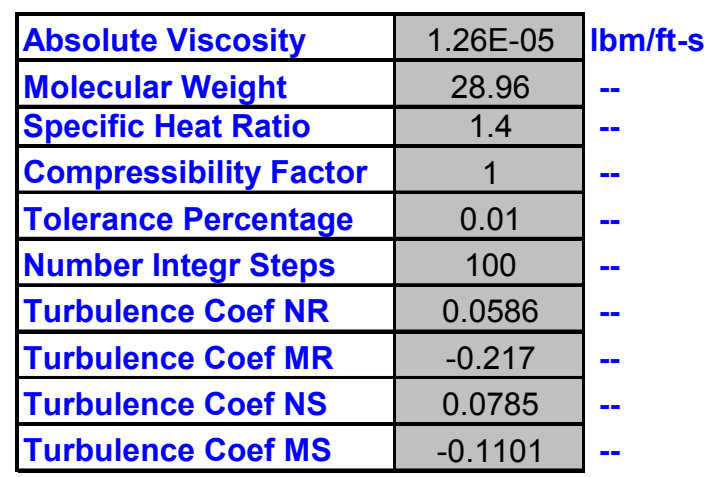

These parameters are used to calculate air flow rates and coefficients. The following figure displays the air flow rate for a single seal. The maximum allowable air flow is $.566 \mathrm{~m}^{3} / \mathrm{s}(1200 \mathrm{SCFM})$, and therefore, the maximum flow for a single seal is $.283 \mathrm{~m}^{3} / \mathrm{s}$ (600 SCFM). The 760 and $1020 \mu \mathrm{m}$ (30 and 40 mil) clearances are only feasible with relatively high backpressure, which would require exit labyrinth seals. The smaller clearances appear to have acceptable flow rates at all back pressures.

Fig. 80 through Fig. 83 display the estimated force levels generated by a single seal. An excitation amplitude of $10 \mu \mathrm{m}(.39 \mathrm{mils})$ is assumed based on previous tests [8]. The damping forces also assume a frequency of $140 \mathrm{~Hz}$, the free-free natural frequency of the rotor. The supply pressure is $1.83 \mathrm{MPa}(265 \mathrm{psia})$. 


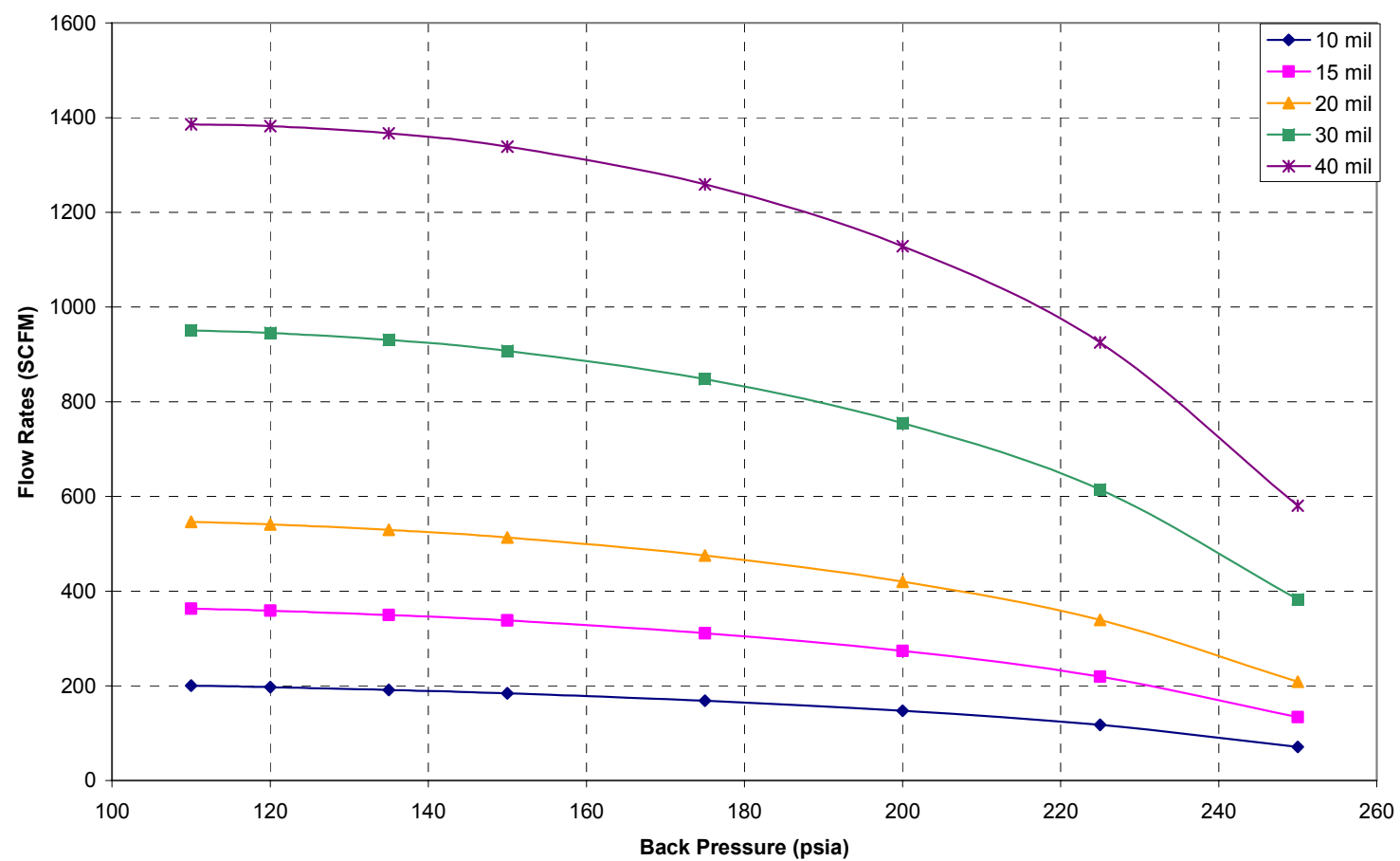

Fig. 80 Air flow rates for a single smooth seal with inlet pressure 1.83 MPa (265 psia)

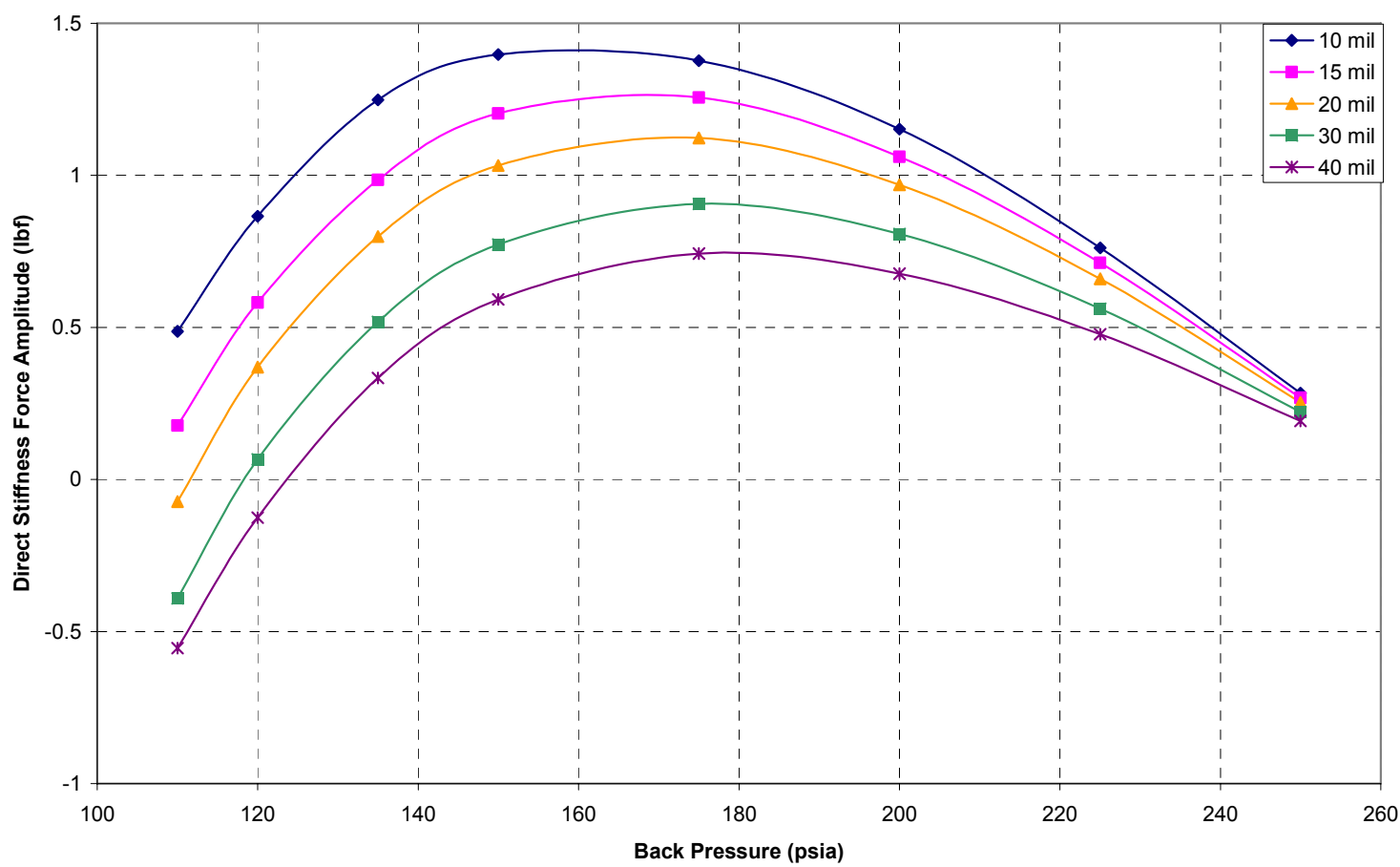

Fig. 81 Air seal average direct stiffness forces for $10 \mu \mathrm{m}(.39 \mathrm{mil})$ shake amplitude 


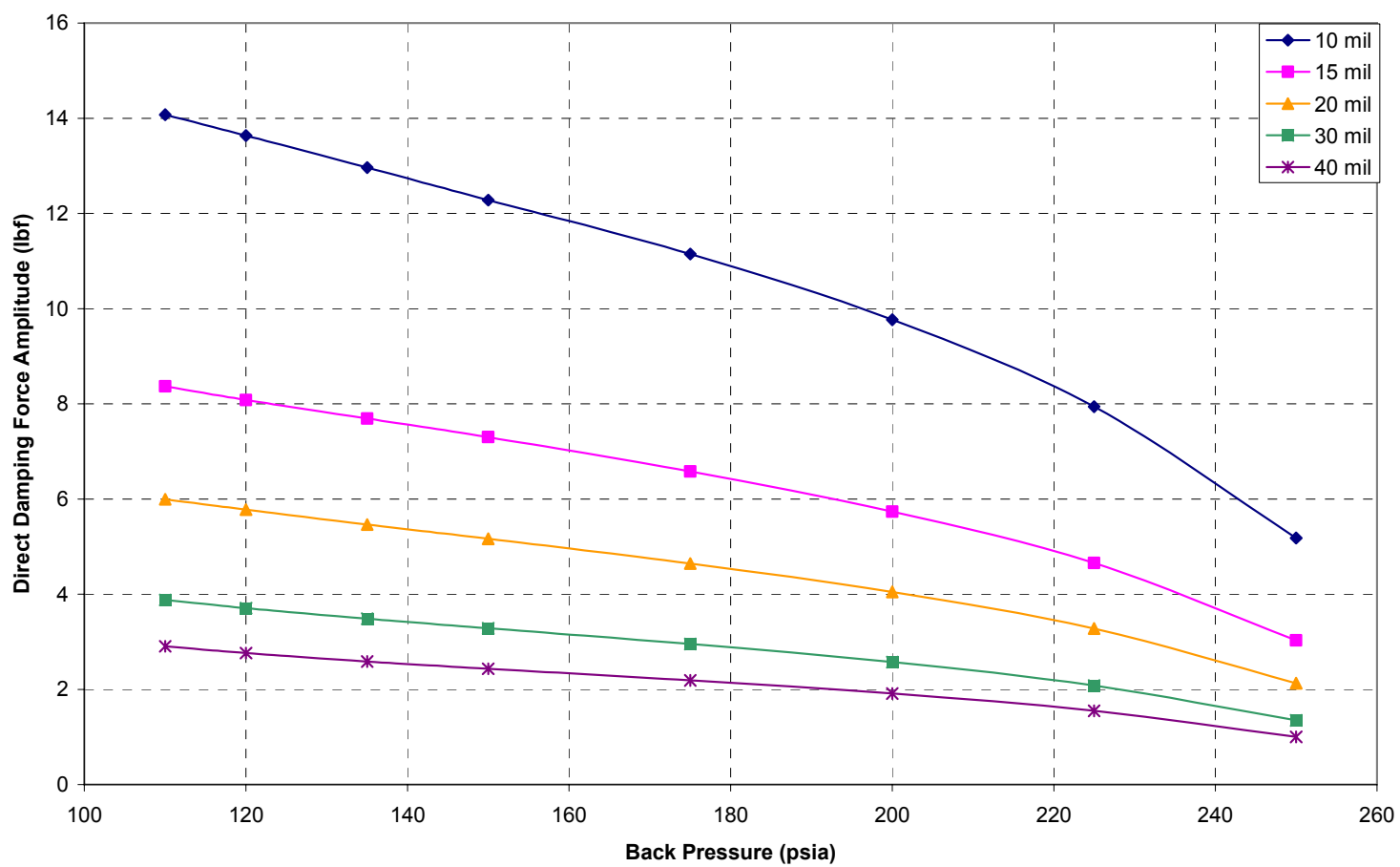

Fig. 82 Air seal average direct damping forces at $140 \mathrm{~Hz}$

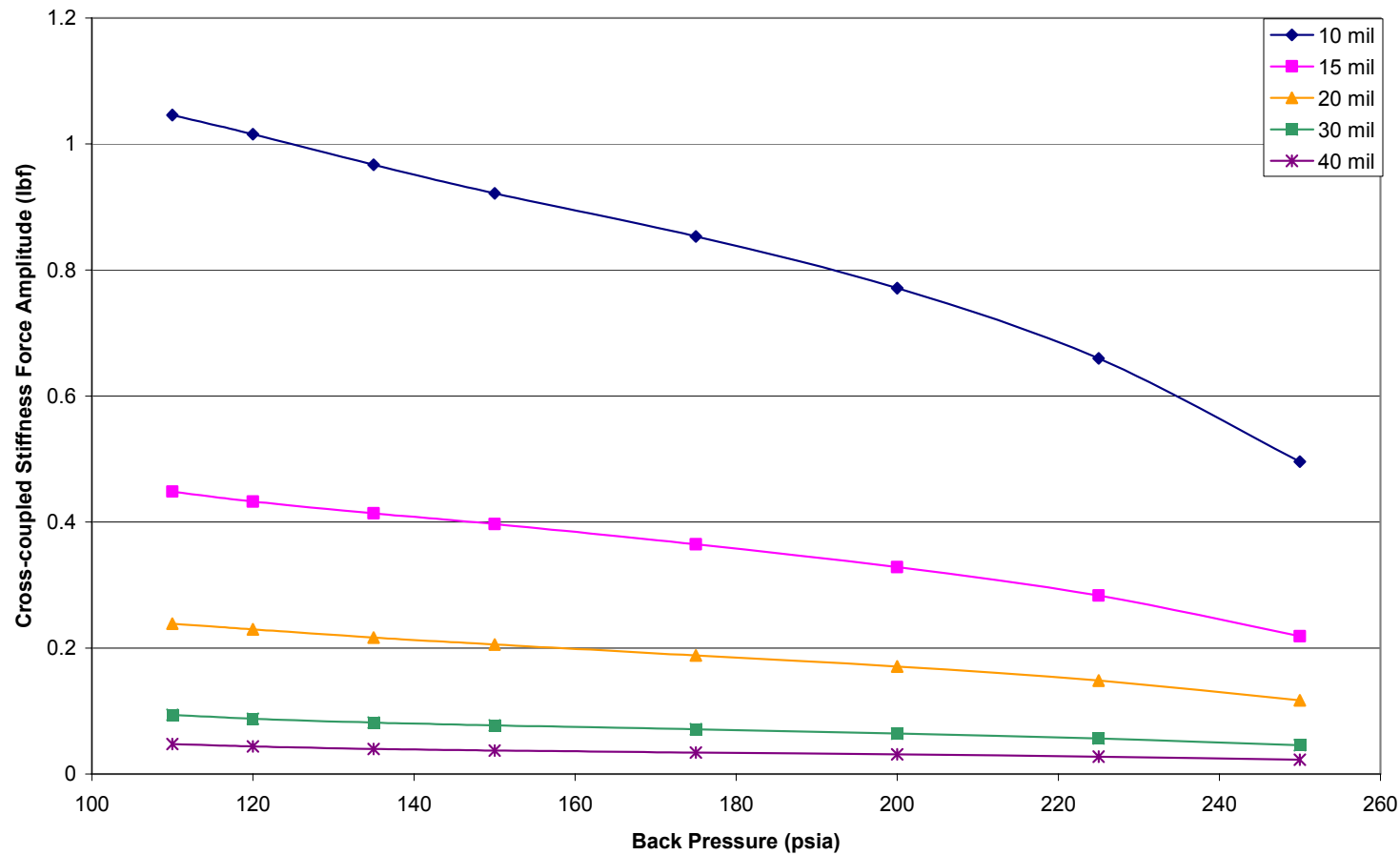

Fig. 83 Air seal cross-coupled stiffness forces for $10 \mu \mathrm{m}(.39$ mil) shake amplitude 
The first conclusion is that a smaller clearance is preferable so long as rotor-seal contact can be avoided. The direct and cross-coupled stiffness terms have a maximum of around 9 to $13 \mathrm{~N}$ ( 2 to $3 \mathrm{lb}_{\mathrm{f}}$ ) for two seals with $250 \mu \mathrm{m}$ (10 mil) clearance. The damping term from two seals has a maximum of $124 \mathrm{~N}\left(28 \mathrm{lb}_{\mathrm{f}}\right)$. The uncertainty of the measurements from previous tests is $0.1 \%$ of load capacity or about $4.4 \mathrm{~N}\left(1 \mathrm{lb}_{\mathrm{f}}\right)$. Therefore, the best possible uncertainty in the seal measurements would be around $50 \%$ for the stiffness terms and 3\% for the damping terms. Additionally, the maxima do not occur at the same back pressure, and a design compromise would result in a reduction of some of the force levels. The primary conclusion is that, given the constraints of $1.83 \mathrm{MPa}$ (265 psia) supply pressure, only the damping term of the air seal can be identified with reasonable accuracy.

\section{Water Seal Analysis}

Back-to-back water seals are also considered as a modification to the magnetic bearing test rig. XLAnSeal, a seal prediction code developed at the Turbomachinery Laboratory, is used to analyze the water seals.

Table 14 displays the parameters used in XLAnSeal. The clearances are 250, 380, 510, $760,1020 \mu \mathrm{m}(10,15,20,30$, and 40 mils $)$. The pressure drop is set to $1.03 \mathrm{MPa}(150$ psia), and the speed is varied from 0 to $8000 \mathrm{rpm}$. The $X L A n S e a l$ values are used for the properties of water, and default code coefficients and factors are used. 
Table 14 Parameters for XLAnSeal

\begin{tabular}{|c|c|}
\hline Seal Diameter & 3.917 \\
\hline Seal Axial Length & 4 \\
\hline Inlet Radial Clearance & 0.04 \\
\hline Exit Radial Clearance & 0.04 \\
\hline Preload & \\
\hline Number of Lobes & 1 \\
\hline Lead Edge Location & 0 \\
\hline
\end{tabular}

\begin{tabular}{|c|c|}
\hline Max Iterations & 199 \\
\hline Momentum Relaxation Facto & 0.9 \\
\hline Pressure Relaxation Factor & 0.6 \\
\hline Temperature Relaxation Fact & 0.9 \\
\hline Rotor Relative Roughness & 0.005 \\
\hline Stator Relative Roughness & 0.01 \\
\hline Moody's Coef Amod & 0.001375 \\
\hline Moody's Coef Bmod & 500000 \\
\hline Moody's Coef Expo & 0.33333 \\
\hline No. Circ. Grid Points & 21 \\
\hline No. Axial Grid Points & 6 \\
\hline
\end{tabular}

\begin{tabular}{|l|c|}
\hline Entrance Loss Coef & 0.2 \\
\hline Exit Seal Coef & 0 \\
\hline
\end{tabular}

\begin{tabular}{|c|c|c|}
\hline Supply Temperature & 80 & ${ }^{0} \mathrm{~F}$ \\
\hline $\begin{array}{l}\text { Selected Lubricant } \\
\text { Water (internal values) }\end{array}$ & & $\nabla$ \\
\hline Viscosity at Tsupply & & \\
\hline Density at Tsupply & & $\mathrm{Ib} / \mathrm{ft}^{3}$ \\
\hline Compressibility & & $\mathrm{in}^{2} / / \mathrm{b}$ \\
\hline Specific Heat & & $\mathrm{BTU} /\left(\mathrm{Ib}^{0} \mathrm{~F}\right)$ \\
\hline Thermal Conductivity & & $B T U /\left(f t-h r^{0} F\right)$ \\
\hline Coef Therm Exp & & $11^{0} \mathrm{~F}$ \\
\hline Temp Visc Coef & & $1 /{ }^{0} \mathrm{~F}$ \\
\hline
\end{tabular}

\begin{tabular}{|c|c|c|}
\hline Inlet Swirl Ratio & 0 & -- \\
\hline X Static Eccentricity & 0 & -- \\
\hline Y Static Eccentricity & 0 & |-- \\
\hline Moment Coef Option & \multicolumn{2}{|c|}{ Do Not Compute } \\
\hline Frequency Analysis Option & \multicolumn{2}{|c|}{ Synchronous Analysis } \\
\hline Constant Shaft Rpm & & spm \\
\hline
\end{tabular}

\begin{tabular}{|l|l|l|}
\hline Static Rotation About X & 0 & radians \\
\hline Static Rotation About Y & 0 & radians \\
\hline Seal Pivot Location & 0 & inches \\
\hline
\end{tabular}

The above parameters are used to calculate flow rate and forces. The following figures display results for a single seal. An available pump is rated for $.0095 \mathrm{~m}^{3} / \mathrm{s}(150 \mathrm{gpm})$ at $1.03 \mathrm{MPa}(150 \mathrm{psi})$. As mentioned previously, $1.03 \mathrm{MPa}(150 \mathrm{psi})$ was used to for calculations, and the acceptable flow rate is $.0047 \mathrm{~m}^{3} / \mathrm{s}(75 \mathrm{gpm})$ or less per seal. A bypass valve can be used to prevent pump damage due to low flow rate.

Fig. 84 displays the water flow rate for a single seal. Fig. 85 through Fig. 88 display the estimated force levels generated by a single seal. An excitation amplitude of $10 \mu \mathrm{m}(.39$ mil) is assumed based on previous tests. The damping and added mass forces also assume a frequency of $140 \mathrm{~Hz}$. 


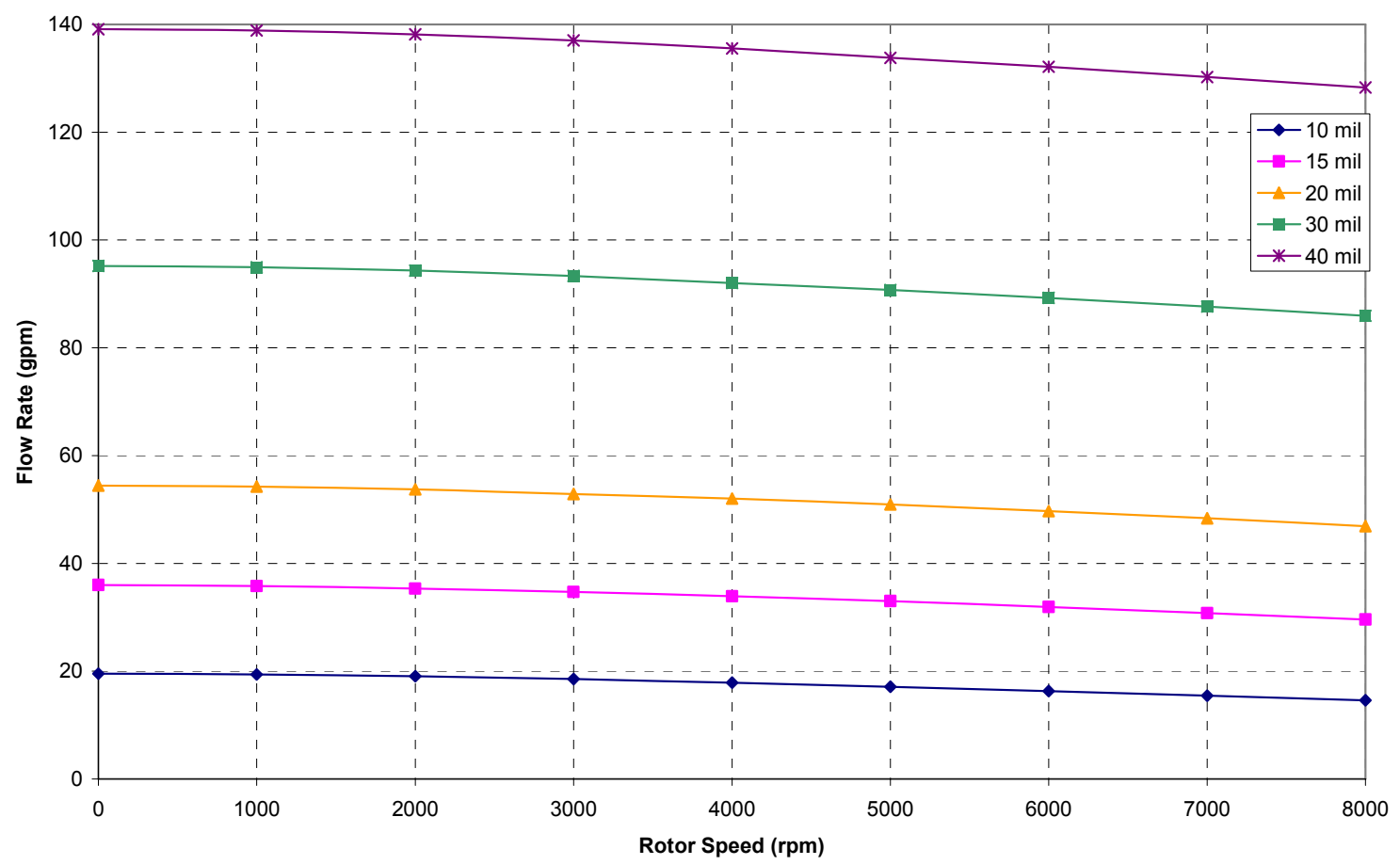

Fig. 84 Water flow rates for a single smooth seal with 1.03 MPa (150 psi) pressure drop

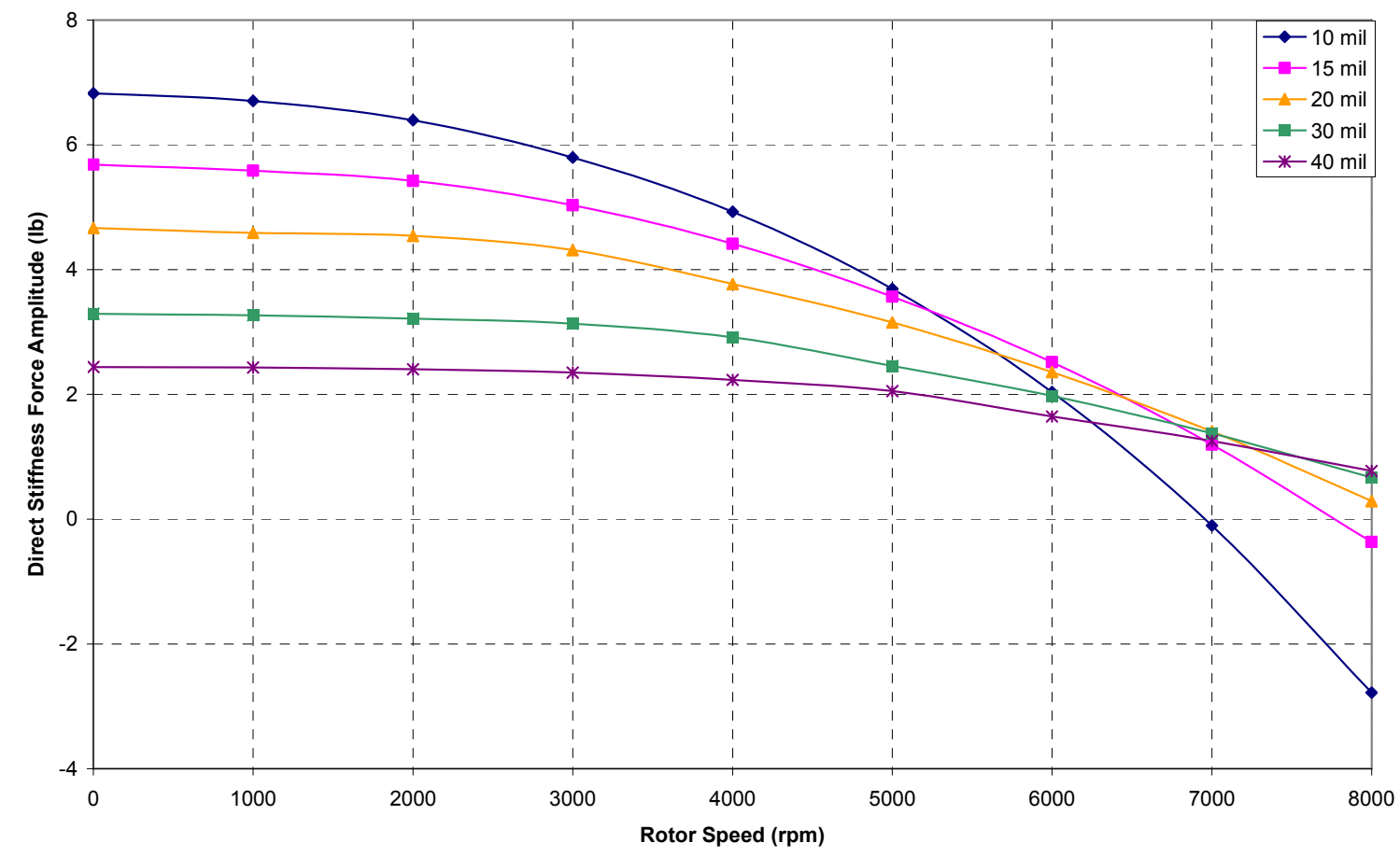

Fig. 85 Water seal direct stiffness forces for $10 \mu \mathrm{m}(.39$ mil $)$ shake amplitude 


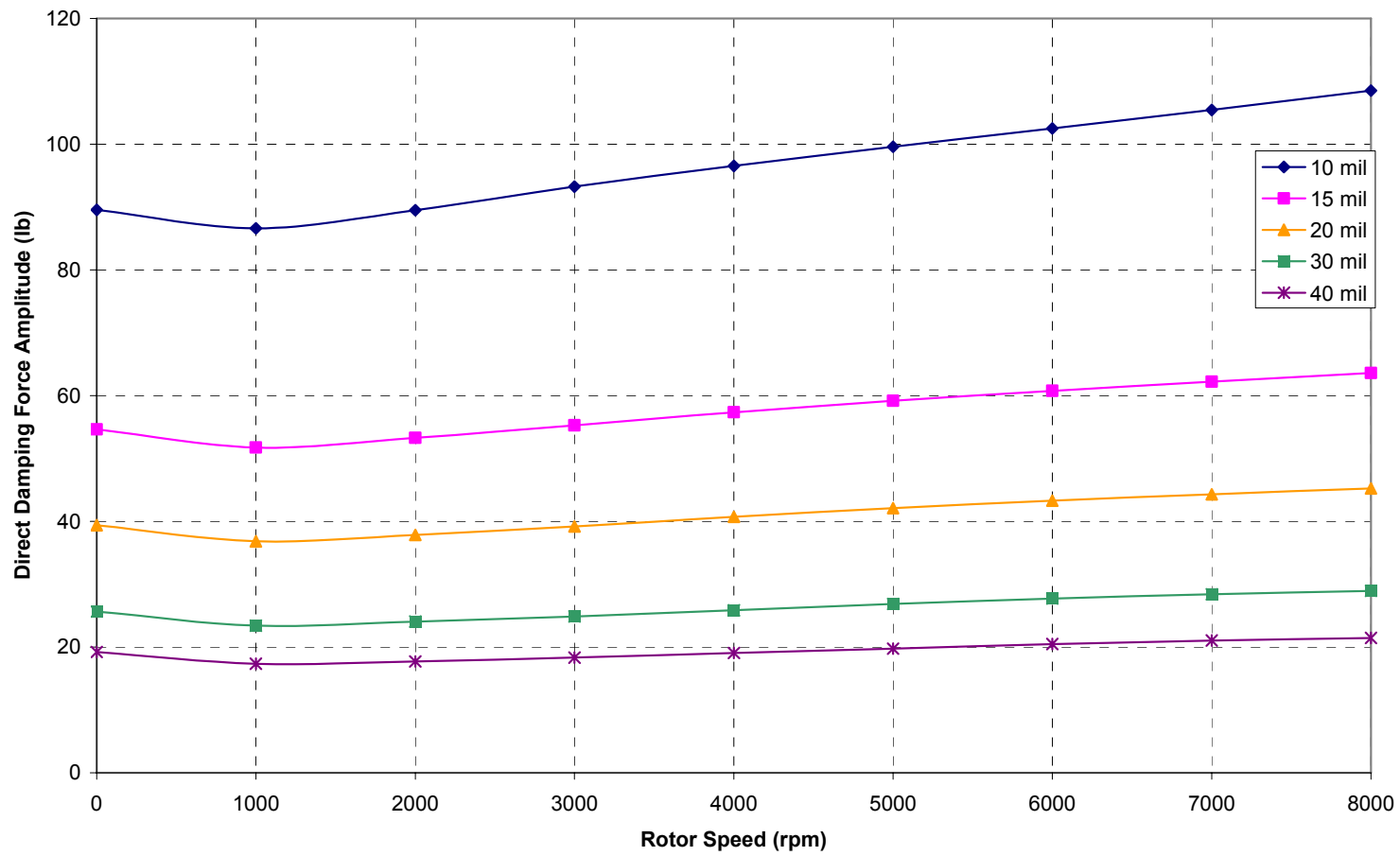

Fig. 86 Water seal direct stiffness forces for $10 \mu \mathrm{m}(.39$ mil) shake amplitude

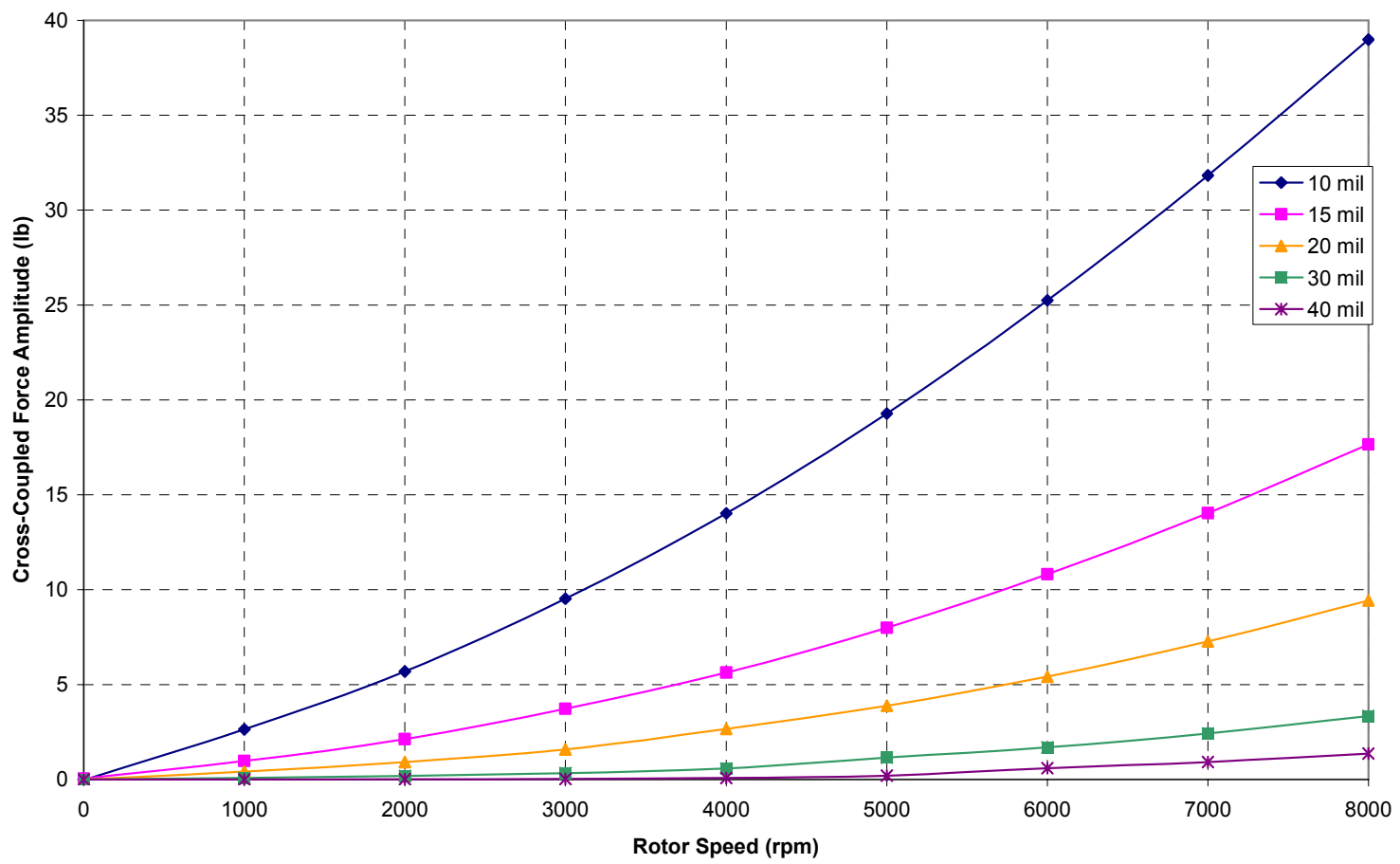

Fig. 87 Water seal cross-coupled stiffness forces for $10 \mu \mathrm{m}(.39$ mil) shake amplitude 


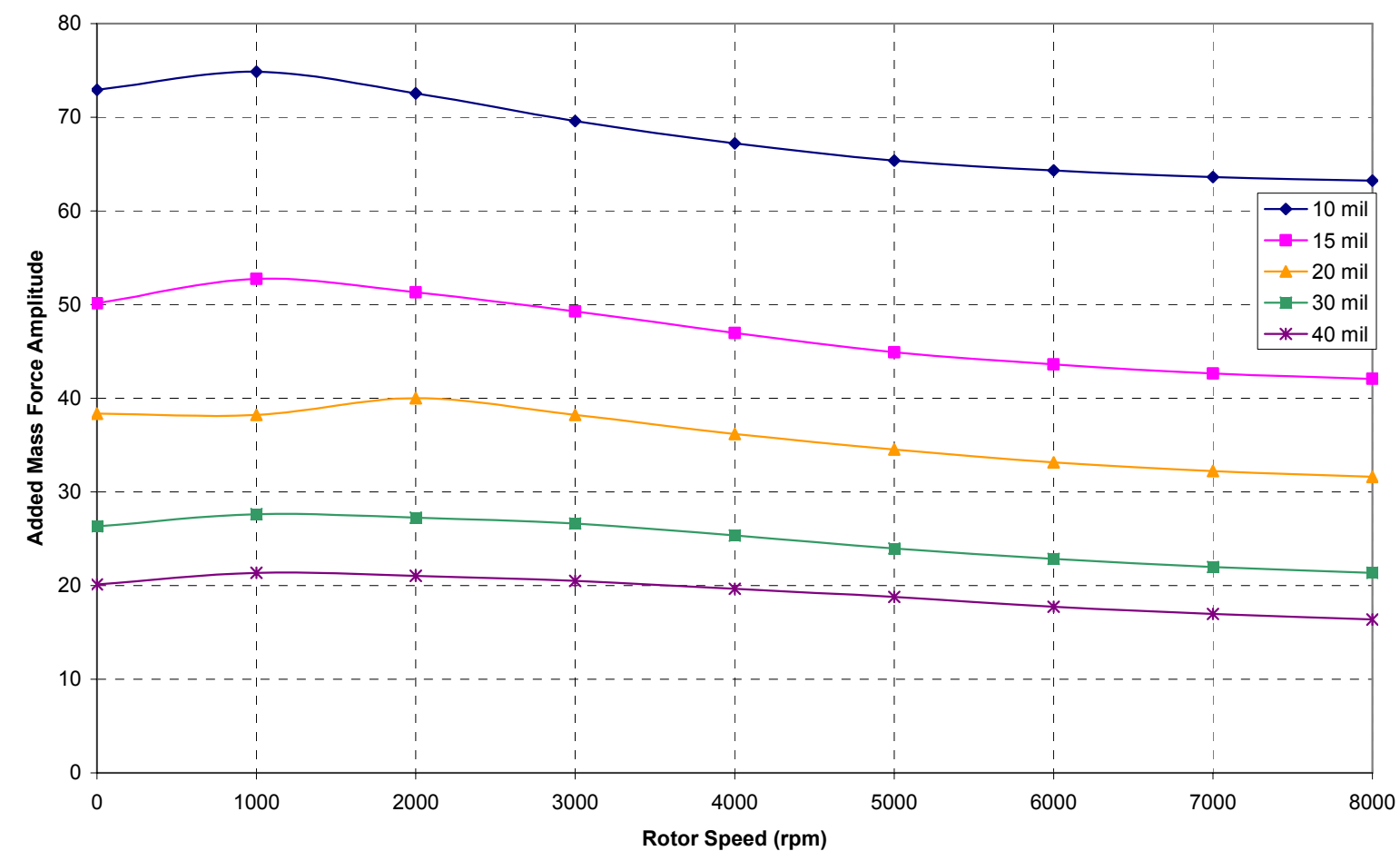

Fig. 88 Water seal added mass forces at $140 \mathrm{~Hz}$

The 760 and $1020 \mu \mathrm{m}$ (30 and $40 \mathrm{mil}$ ) cases require more flow than is achievable with the pump specifications. In addition, reducing the clearance increases the force levels, which produces better signal to noise ratios. For the $510 \mu \mathrm{m}(20 \mathrm{mil}) \mathrm{case}$, the stiffness terms have uncertainties from 5 to $10 \%$, and the damping and added mass terms would have uncertainties of around $1 \%$. Further reduction in the clearance improves the uncertainties.

\section{Seal Selection}

While the water seal clearly offers benefits related to the force levels generated by the seal, it also has drawback such as additional cost and time to completion. The air seal force levels for the damping terms are adequate, and the force levels for the stiffness terms are potentially identifiable as well. The seal design can also include swirl races to increase the cross-coupled stiffness force levels. This analysis indicates that air seals 
will generate sufficient force levels for identification. Accordingly, the air seals are selected for performing parameter identification.

\section{Seal Design FEA for Strength and Sealing}

The purpose of the present finite element analysis (FEA) is to determine the stresses and deflections encounter during operating conditions for the air seal design. The analysis is performed using COSMOS Works.

\section{The Model and Constraints}

The housing and seal model is displayed below. Fig. 89 shows a conceptual model of the air seal hardware. The major components include upper and low housings machined from A36 steel and the seal inserts made from 6061 Aluminum. The significant features are the bore diameter of the seal inserts, and the housing bolt holes.

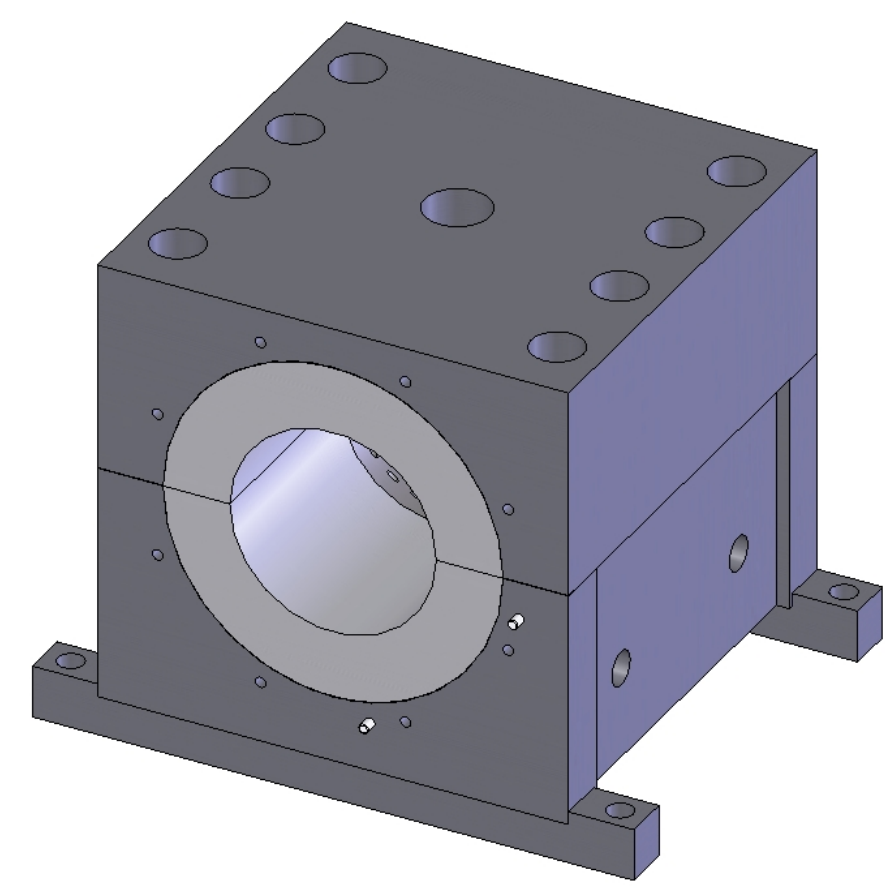

Fig. 89 Air seal and housing assembly 
For the purpose of FEA, the geometry is simplified by removing the smaller bolt holes and pins. The model is also sliced along two vertical planes of symmetry to allow for denser meshing and reduced computational requirements. Two views of the section are displayed below.
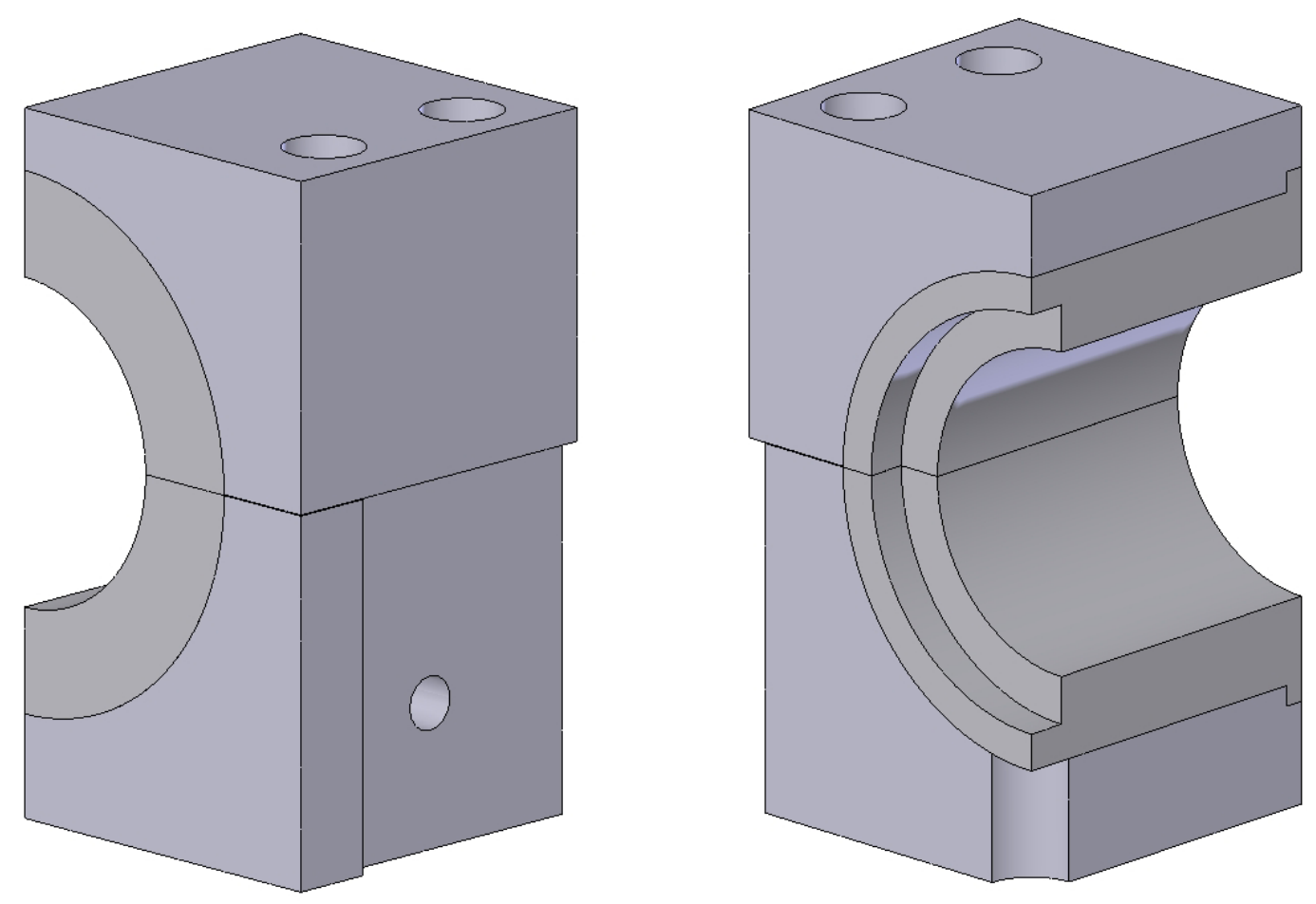

Fig. 90 Simplified air seal and housing for FEA

The mesh is shown in left assembly of Fig. 91. The right assembly shows the planar constraints associated with the two planes of symmetry. These constraints prohibit motion perpendicular to the plane. 

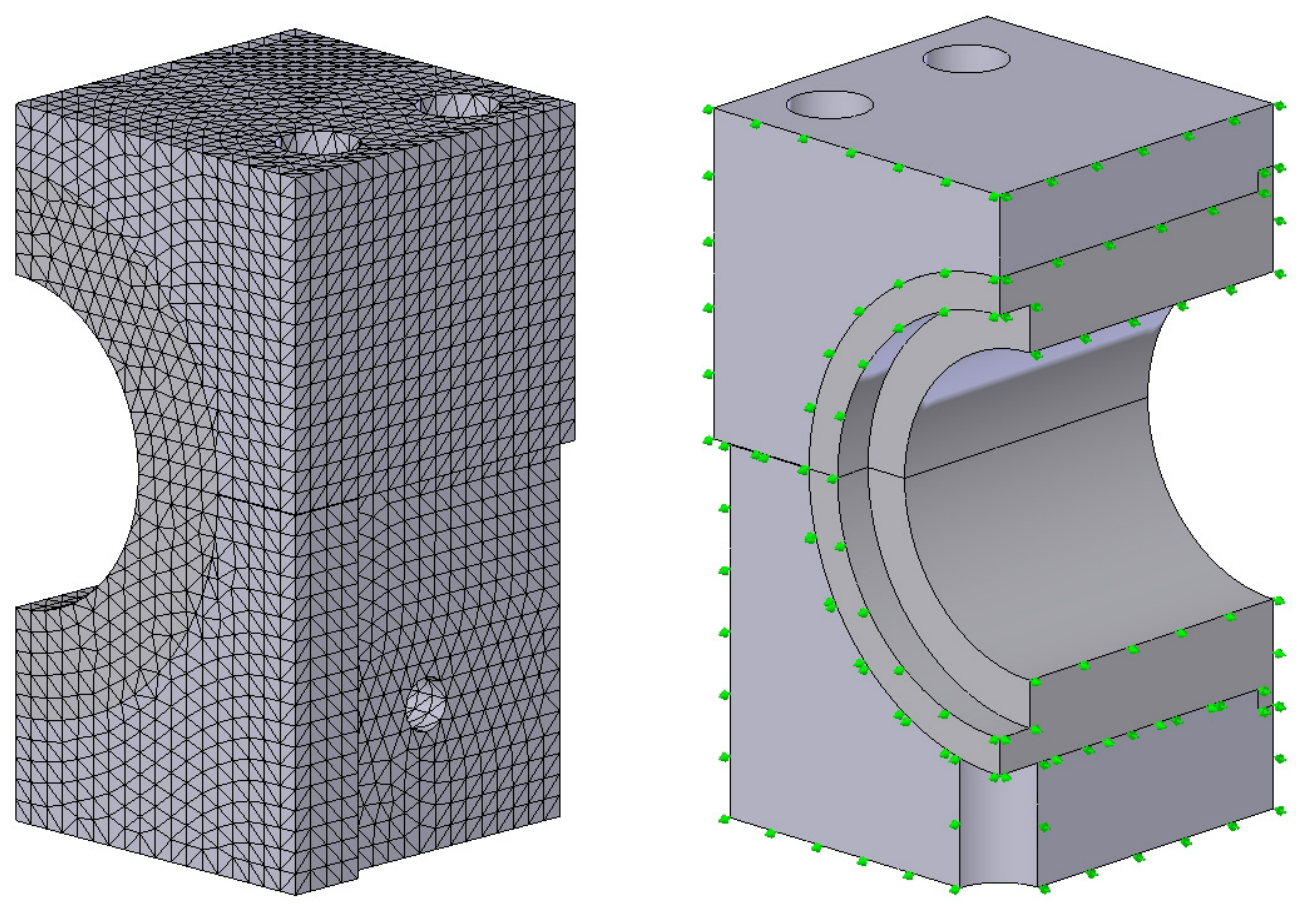

Fig. 91 FEA mesh and symmetry constraints

The model uses default properties from the Solid Works Materials Library. The housing material is 1020 steel (similar properties to A36), and the seal material is 6061 aluminum. COSMOSWorks has several options for the contact interfaces between assembled parts. The option applicable for this situation is "Node to Node" contact. This applies compressive loads between parts and shear loads when friction is include in the analysis.

\section{Results with No Clamp Load}

For the first scenario, the bolts are not torqued to exert a clamp load. The upward facing surfaces of the counterbores are constrained to prevent motion perpendicular to the surface, and the threaded surfaces are constrained to prevent axial motion. A uniform pressure of $1.72 \mathrm{MPa}(250 \mathrm{psi})$ is applied to the inner surfaces of the seals. The constraints and pressure are displayed in the figure below. 

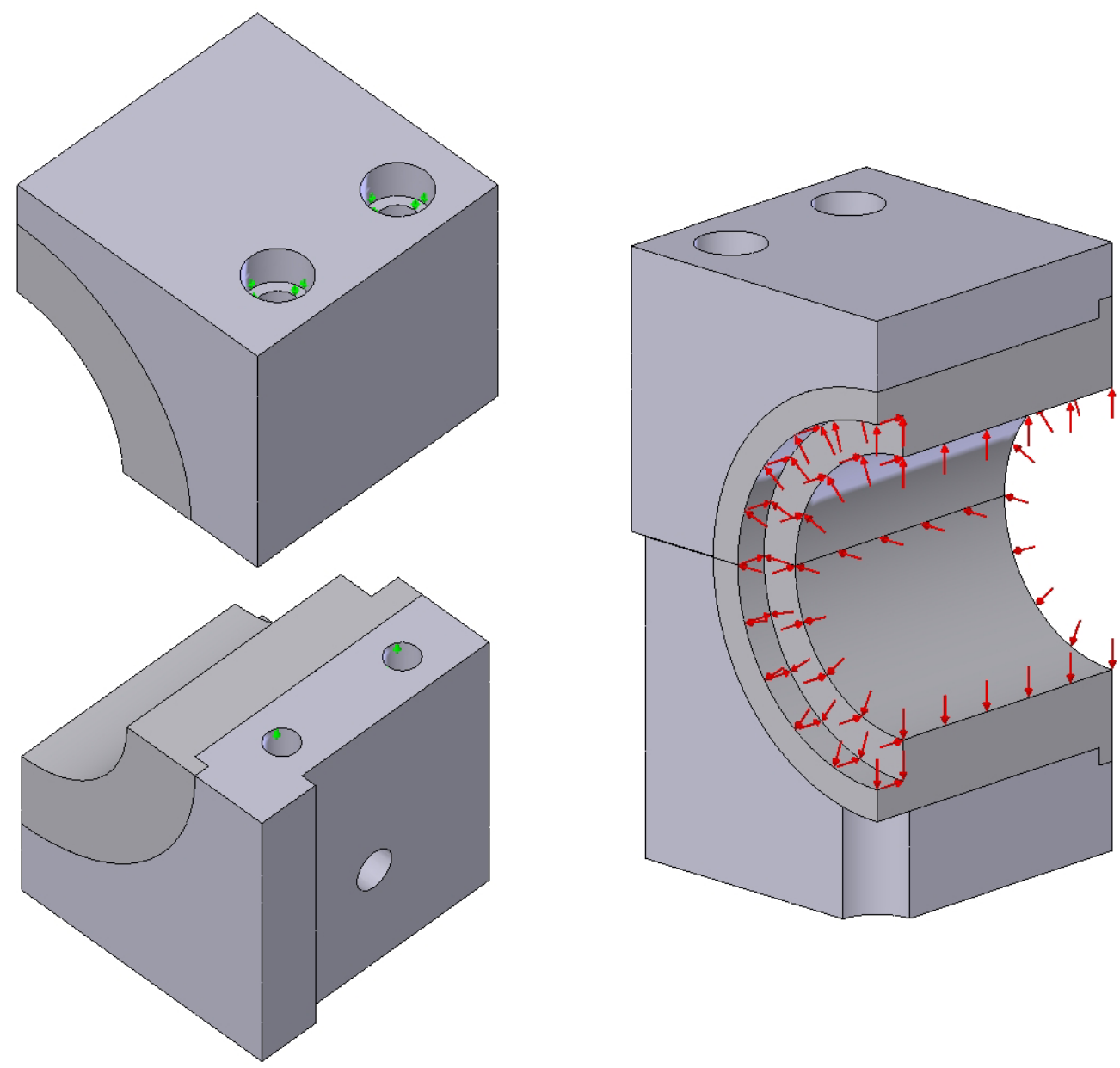

Fig. 92 FEA bolt-hole constraints and pressure load

The FEA is competed and the deflections of the assembly are displayed below. These results show that the seals and housing deflect to allow leakage if the housing bolts are not torqued. The maximum deflection is $3.6 \mu \mathrm{m}(.14 \mathrm{mil})$. 

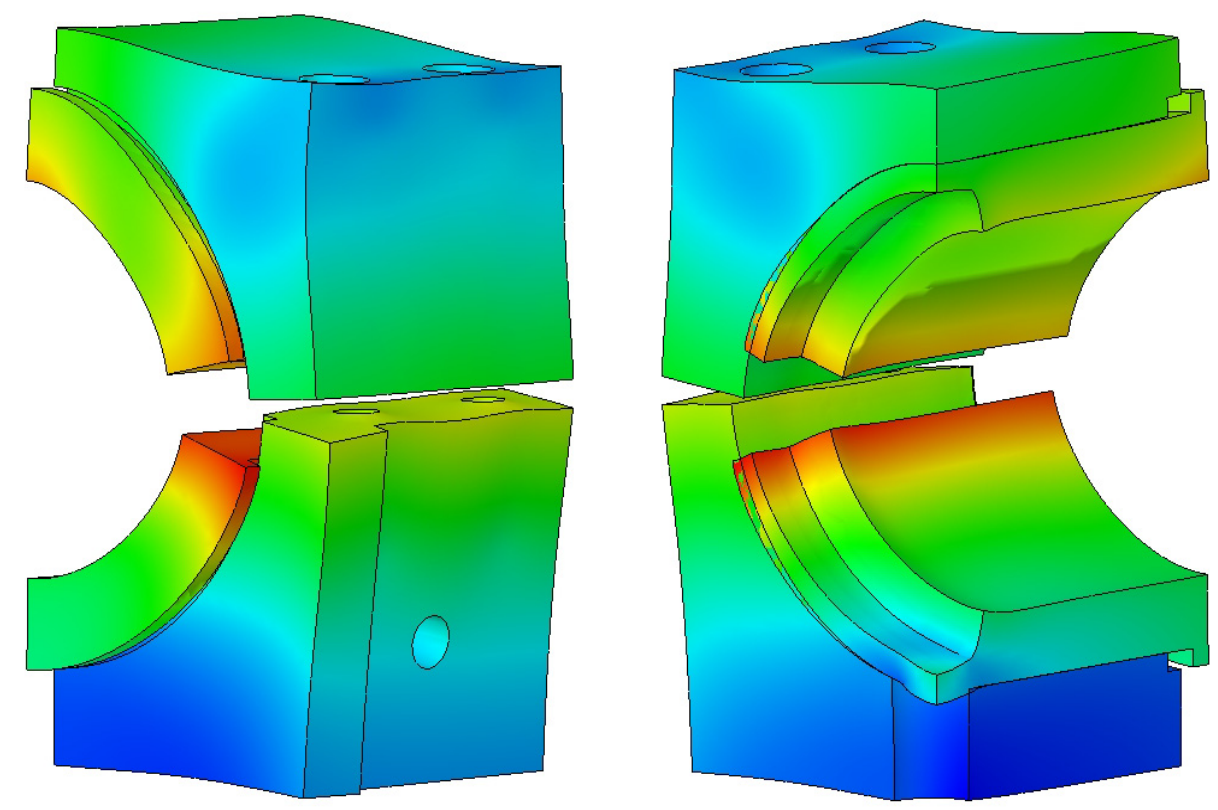

Fig. 93 FEA deflection results with displacement constraints

\section{Results with Clamp Load}

The clamp load can be calculated using equations for the Machinery's Handbook ${ }^{1}$. The applied torque $T$ is equal to the product of the clamping force $F$, the torque coefficient $K$, and the nominal bolt diameter $d$.

$$
T=F \cdot K \cdot d
$$

The torque coefficient is a function of the nominal diameter, the thread pitch $P$, the thread coefficient of friction $\mu_{s}$, the pitch diameter $d_{2}$, the flank angle $\alpha^{\prime}$, the bolt head coefficient of friction $\mu_{w}$, and the equivalent bolt head diameter $D_{w}$.

\footnotetext{
${ }^{1}$ Oberg, Erik, Franklin D. Jones, Holbrook L. Horton, and Hery H. Ryffel. Machinery's Handbook, 26th Edition. Industrial Press Inc.: New York, 2000.
} 


$$
K=\frac{1}{2 d}\left(\frac{P}{\pi}+\mu_{s} d_{2} \sec \alpha^{\prime}+\mu_{w} D_{w}\right)
$$

The equivalent diameter is calculated from the outer diameter of the bolt head $D_{o}$ and the diameter on the bolt head corresponding edge of the bolt head fillet $D_{i}$.

$$
D_{w}=\frac{2}{3}\left(\frac{D_{o}^{3}-D_{i}^{3}}{D_{o}^{2}-D_{i}^{2}}\right)
$$

The flank angle is calculated from the thread half angle $\alpha$ and the thread helix angle $\beta$.

$$
\tan \alpha^{\prime}=\tan \alpha \cos \beta
$$

The thread helix angle is a function of the lead $l$ and the nominal radius $r$.

$$
\tan \beta=\frac{l}{2 \pi r}
$$

The table below contains the parameters for a 5/8-11 UNRC 3A socket head cap screw.

Table 15 Parameters for clamp load calculation

\begin{tabular}{|l|l|l|}
\hline Nominal Diameter & $d$ & 0.625 in. \\
\hline Thread Pitch & $P$ & 0.0909 in. \\
\hline Thread COF & $\mu_{s}$ & 0.15 \\
\hline Pitch Diameter & $d_{2}$ & 0.564 in. \\
\hline Bolt Head COF & $\mu_{w}$ & 0.15 \\
\hline Outer Diameter $=$ & $D_{o}$ & 0.930 in. \\
\hline Inner Diameter & $D_{i}$ & 0.689 in. \\
\hline Thread Half Angle & $\alpha$ & 30 deg. \\
\hline Thread Lead & $l$ & 0.0909 in. \\
\hline Nominal Radius & $r$ & 0.3125 in. \\
\hline
\end{tabular}


The previous equations show that applying a $27 \mathrm{~N}-\mathrm{m}\left(20 \mathrm{ft}-\mathrm{lb}_{\mathrm{f}}\right)$ torque will result in a $8900 \mathrm{~N}\left(2000 \mathrm{lb}_{\mathrm{f}}\right)$ clamp load. These equations are also used to calculate torques that would result in clamp loads of $60 \%$ and $90 \%$ of the proof load. Torques of 230 and 344

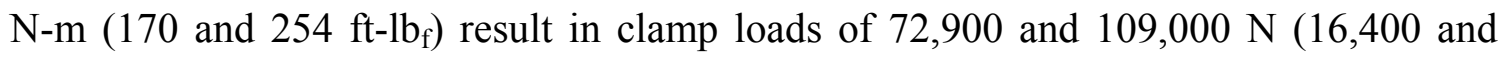
$24,500 \mathrm{lb}_{\mathrm{f}}$ ) respectively. These results agree with the results from Nucor Corporation literature $^{2}$ of 72,500 and 108,000 N (16,300 and 24,400 $\left.\mathrm{lb}_{\mathrm{f}}\right)$.

The following results were obtained using a $8900 \mathrm{~N}$ (2000 lbf) clamp load per bolt applied to the upward facing surface of each counterbore.
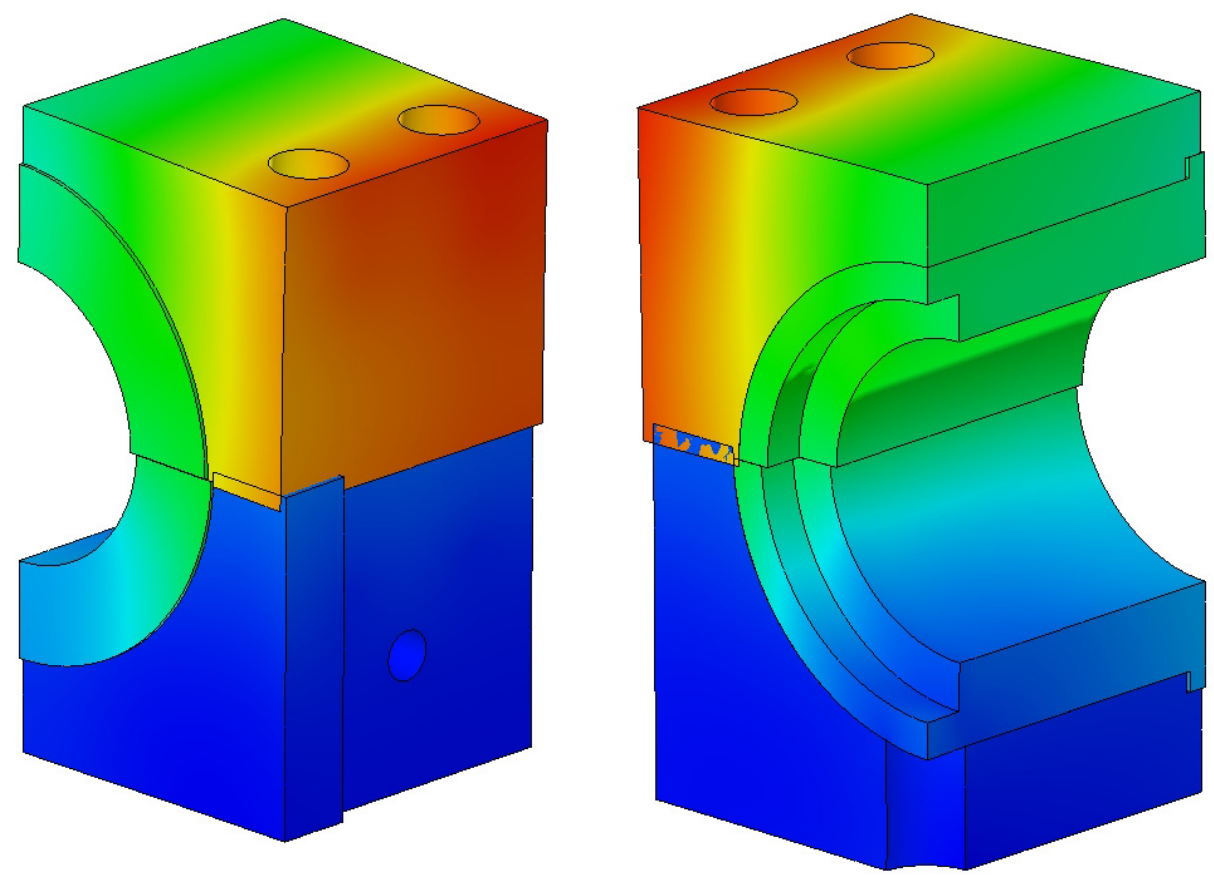

Fig. 94 FEA deflection results with preload

\footnotetext{
${ }^{2}$ Nucor Corporation, 2005, “Cap Screws,” http://www.nucor-fastener.com/pdf/009\%20a.pdf
} 

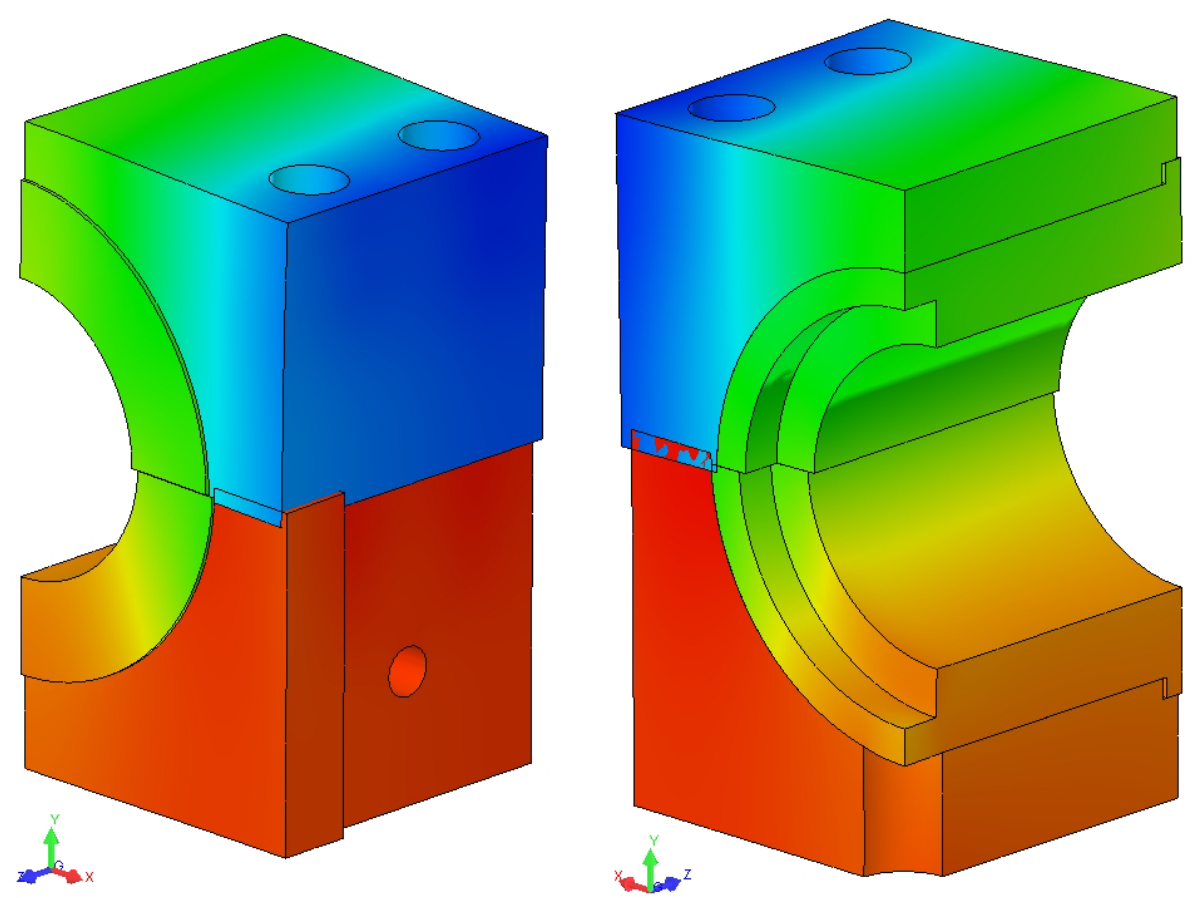

Fig. 95 FEA Y-direction deflection results with preload
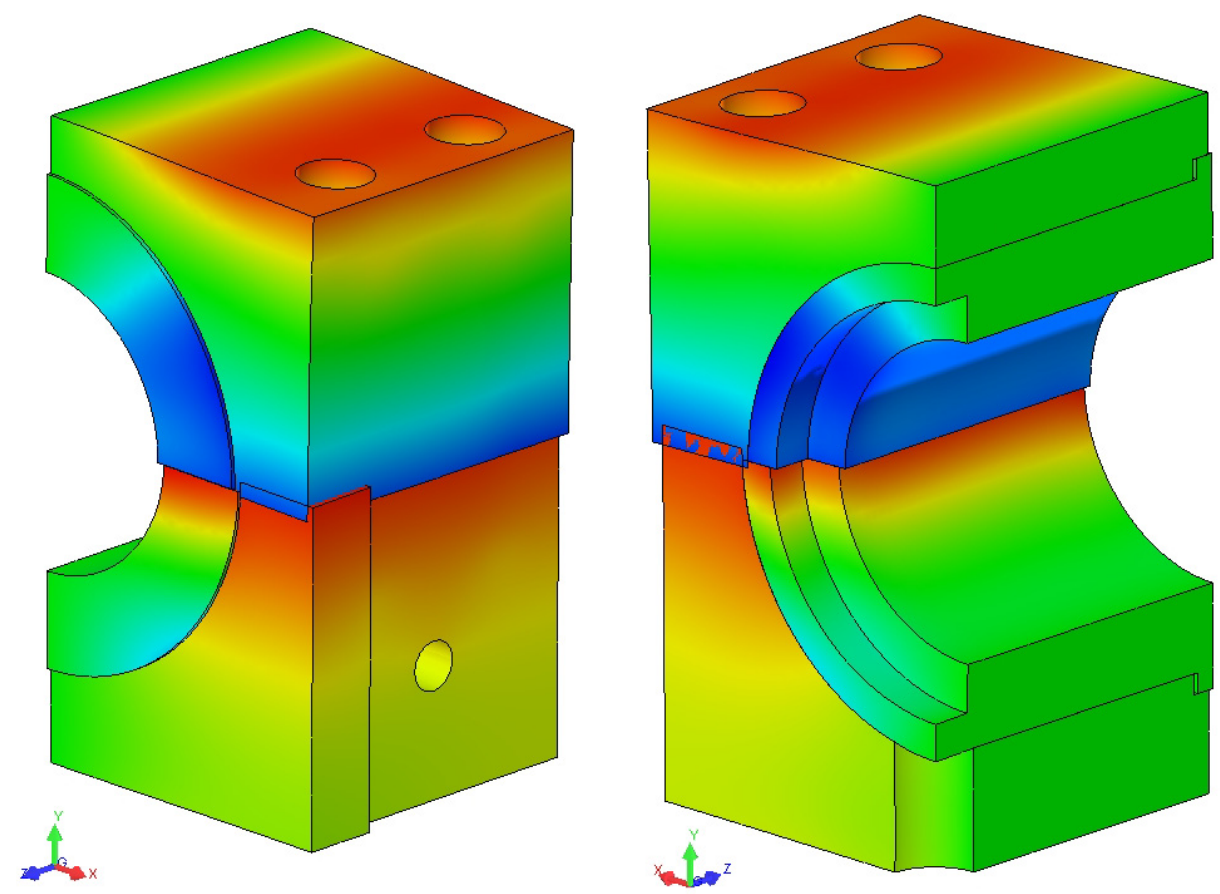

Fig. 96 FEA X-direction deflection results with preload 
It can be seen that the seal halves remain in contact. It should be noted that the housing halves only appear to intersect (because of the amplification factor of the deflection). The clearance between housing halves is much larger than the deflection. The seal surfaces slip relative to one another by roughly $5 \mu \mathrm{m}$ (0.0002 inches). In reality this motion would be limit be the seal pins. This could potentially damage the seal pins. A final analysis is conducted to determine whether this is a critical issue.

\section{Results with Clamp Load and Global Friction}

The final analysis is identical to the previous except for the inclusion of a global friction coefficient. The coefficient of friction is set to 0.15 as a conservative value. The results from this analysis are displayed below.
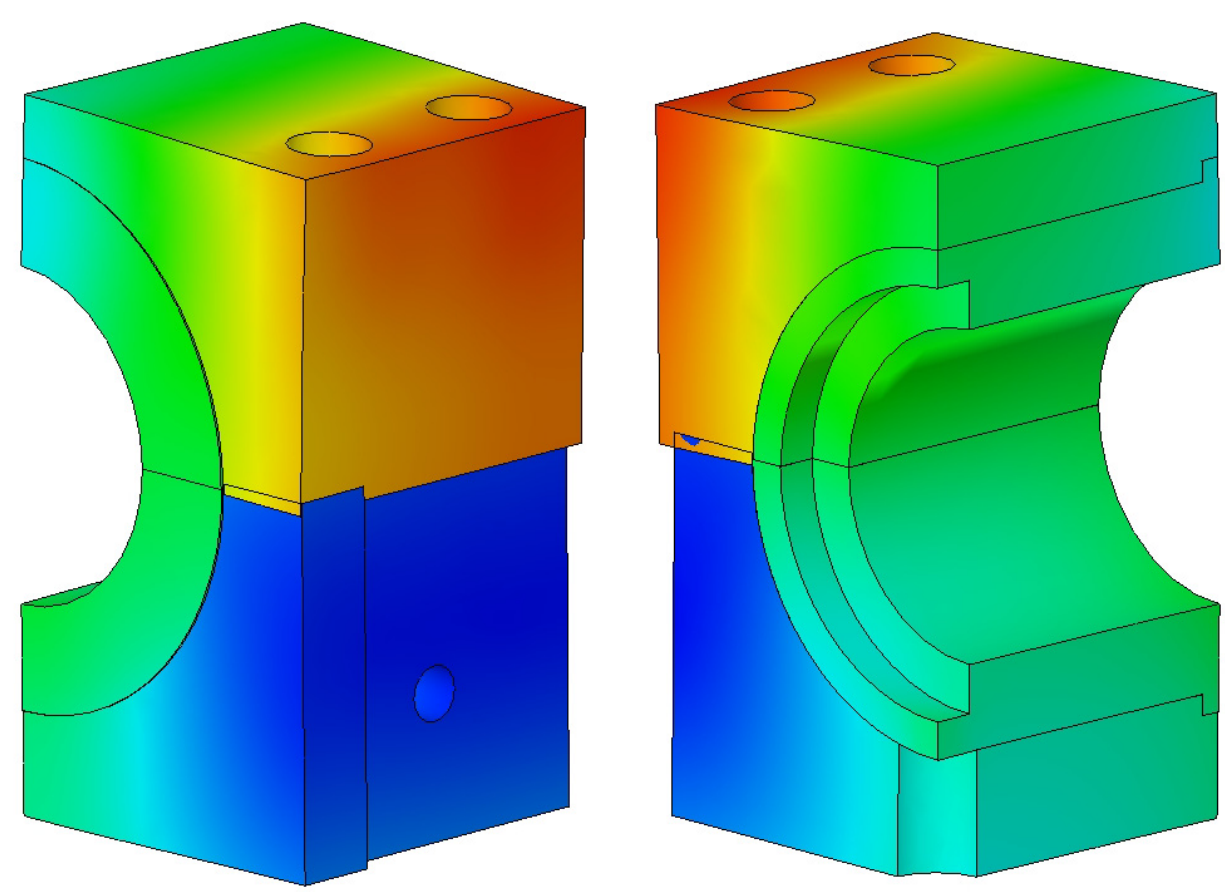

Fig. 97 FEA deflection results with preload and global friction 

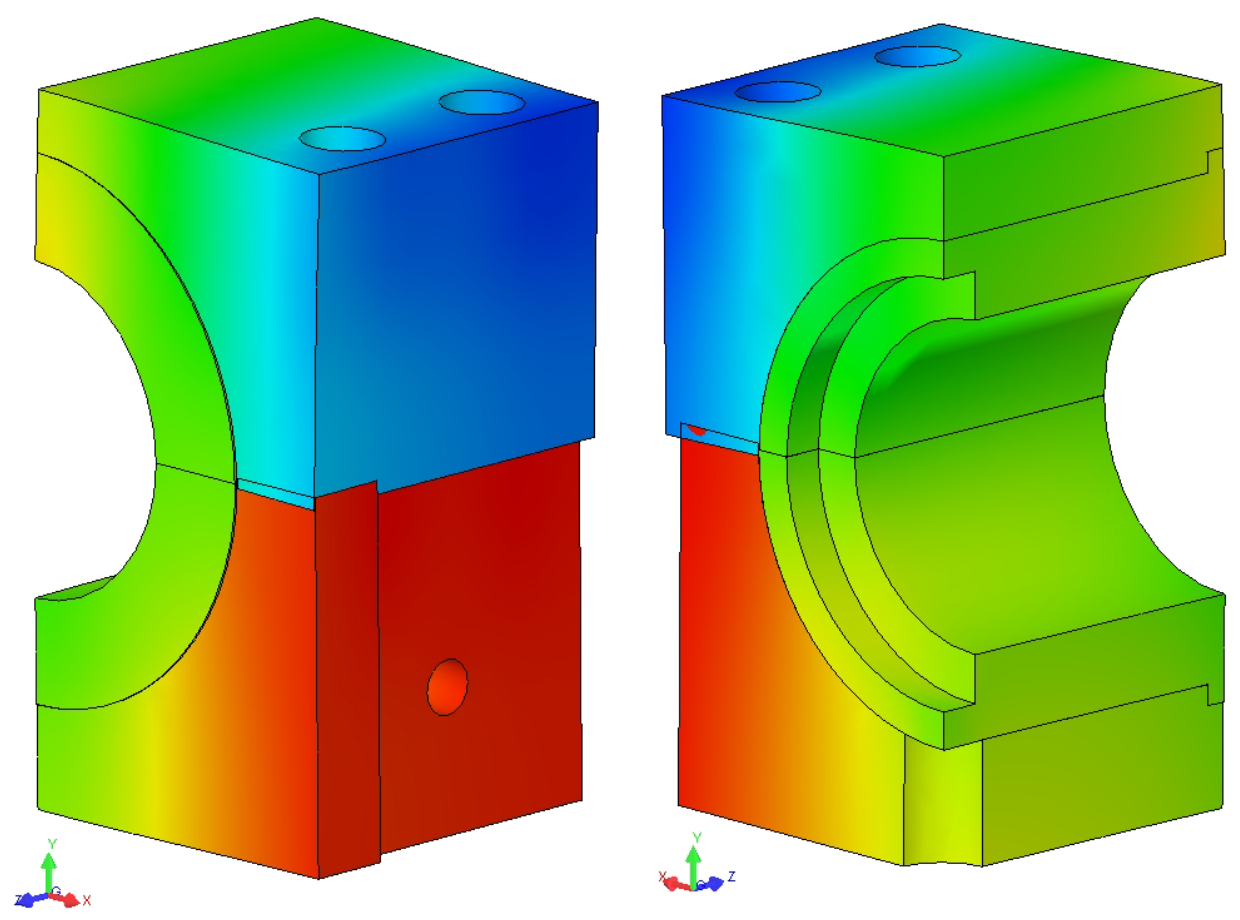

Fig. 98 FEA Y-direction deflection results with preload and global friction
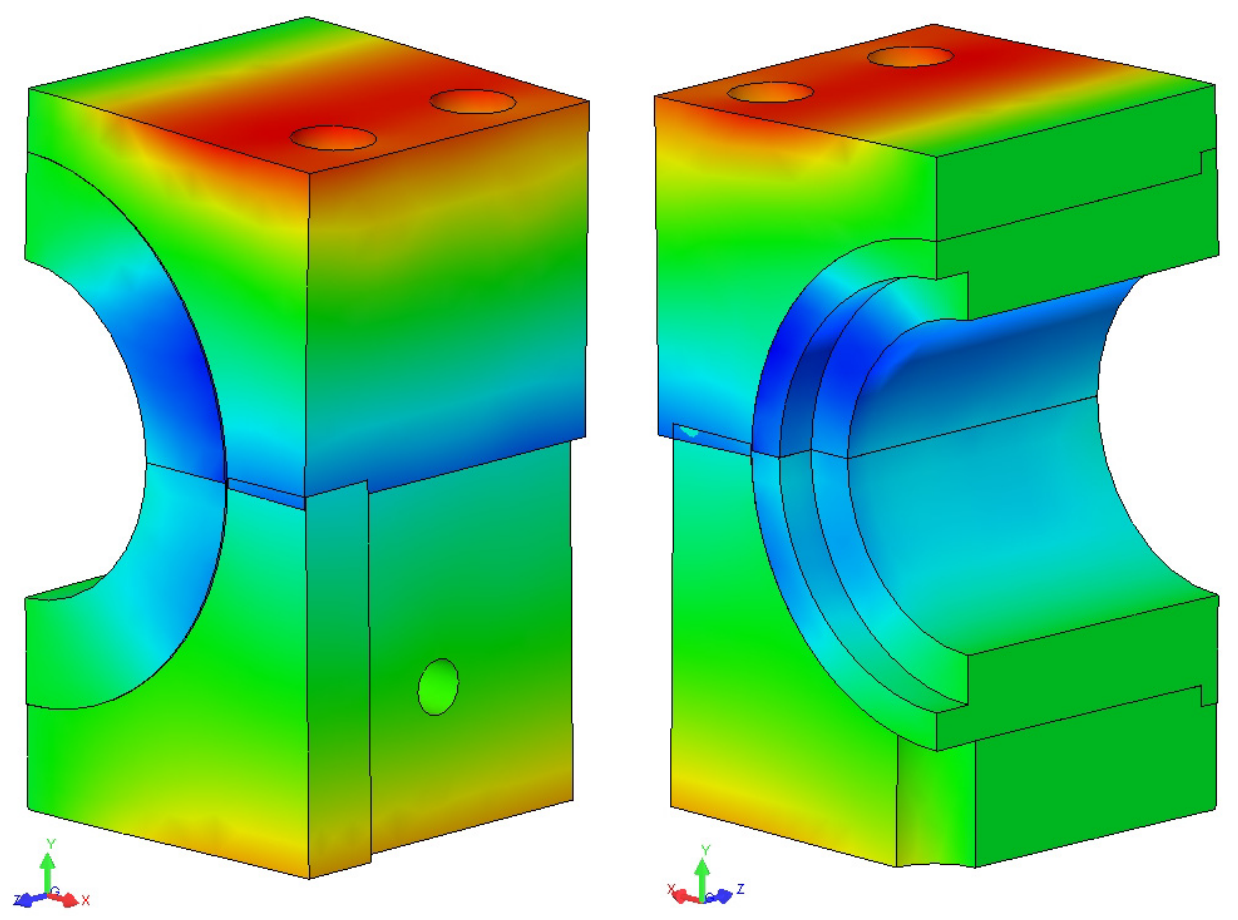

Fig. 99 FEA X-direction deflection results with preload and global friction 
The seals again maintain contact. With the inclusion of global friction, slippage between seals is prevented. The conclusion is that the seal pins will not be damaged.

The von Mises Stresses are displayed below. The maximum stress of $34 \mathrm{MPa}$ (5000 psi) occurs at the counterbores. This value is not considered to be highly accurate because of the limitations of the model geometry. However, the minimum safety factor achieved is 10 , suggesting that the design provides more that sufficient structural integrity.

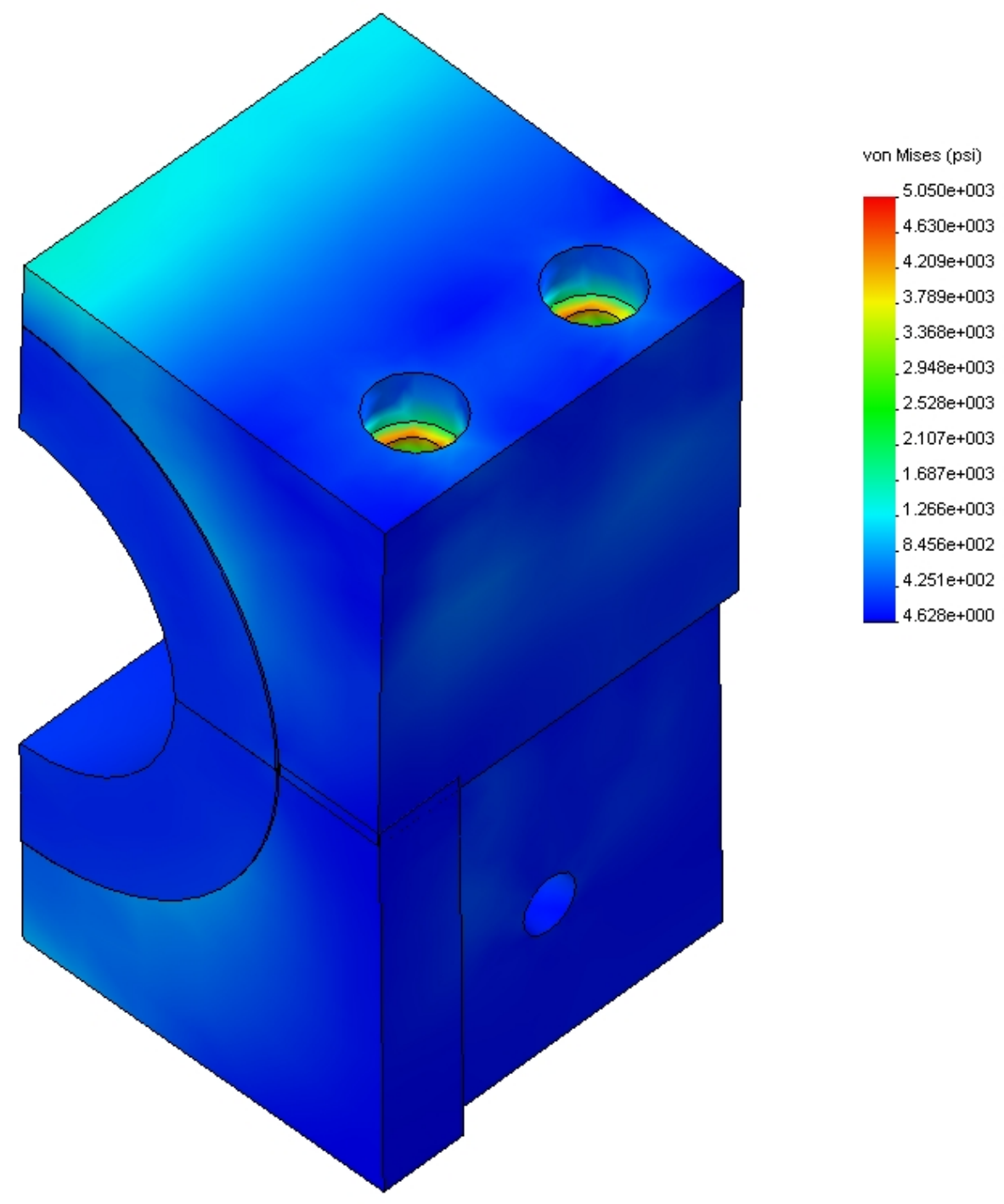

Fig. 100 FEA von Mises stress results with preload and global friction 


\section{Rotordynamic Stability Analysis}

In addition to the FE strength analysis, rotordynamic analysis of the test rig is necessary to ensure stable operation. This analysis predicts the rotordynamic stability of the test rig with the addition of smooth seals.

\section{Seal Models}

Preliminary analysis reveals that a design incorporating only smooth seals will result in choked flow conditions. This would have a negative effect on the ability to predict seal coefficients for the purpose of comparison with test results. Accordingly, the current design now incorporates single-tooth labyrinth seals at the exits of the smooth seals.

A radial clearance of $300 \mu \mathrm{m}$ (0.012 in.) was used for both the smooth and the labyrinth seals. This clearance exceeds the auxiliary bearing clearance of $250 \mu \mathrm{m}(0.010 \mathrm{in}$.) and a bump seal clearance $200 \mu \mathrm{m}(0.008$ in.).

The XLIsotSl software was used to model the smooth seal. Leakage and pressure drop are computed and used in an iterative process with the labyrinth seal predictions. The rotordynamic coefficients are then use to model the smooth seal forces acting on the center of MB test rig rotor.

The XLLaby software computes leakage, pressure drop, and seal coefficients for a seal with a minimum of 2 teeth. The leakage from XLLaby was compared with calculations using the leakage equation for a single-tooth seal. The leakages calculated using these two methods deviated by less than $2 \%$. The labyrinth seal forces are neglected in the test rig model because the seals are expected to function as single-tooth labyrinth seals. By adjusting the smooth seal exit pressure and the labyrinth seal inlet pressure to obtain equivalent flow rates, the following operating conditions were obtained. 
Table 16 Operating conditions for rotordynamic analysis

\begin{tabular}{|l|l|}
\hline Total Air Flow Rate & 350 SCFM \\
\hline Smooth Seal Inlet Pressure & 265 psia \\
\hline Smooth Seal Exit Pressure & 215 psia \\
\hline Smooth Seal Exit Mach Number & 0.22 \\
\hline Labyrinth Seal Inlet Pressure & 215 psia \\
\hline Labyrinth Seal Exit Pressure & 14.4 psia \\
\hline
\end{tabular}

\section{Rotor System Model and Results}

The seal forces from the smooth seal models are applied to the center of FE rotor model. The model displayed below is developed in $X L T R C^{2}$, FE software package developed at the Turbomachinery Laboratory. As shown below, the smooth seal forces are applied 2.5 in. to either side of the midpoint between the two discs.

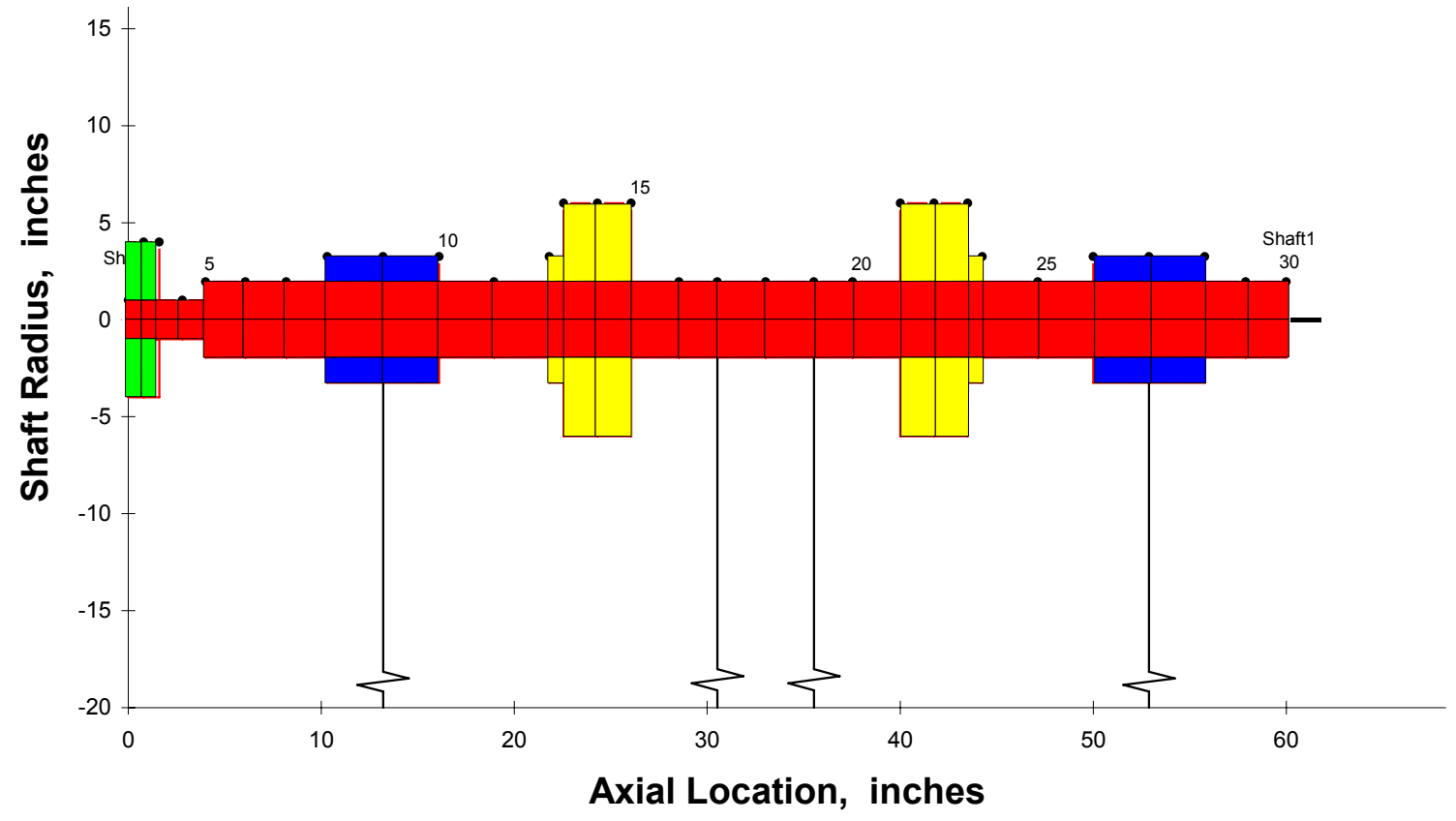

Fig. 101 XLTRC model for rotordynamic analysis 
The eigen-analysis results are interpreted by comparing between the models with and without seals. The case with seals is analyzed for 0 and 0.3 preswirl at the inlet to the smooth seals.

Table 17 Damping ratios and damped natural frequencies for different seal configurations

\begin{tabular}{|c|c|c|c|c|c|c|c|c|c|c|c|}
\hline \multicolumn{4}{|c|}{ Test Rig with No Seals } & \multicolumn{4}{|c|}{ Test Rig with Seals } & \multicolumn{4}{|c|}{ Test Rig with Seals and Preswirl } \\
\hline \multicolumn{2}{|c|}{$10 \mathrm{rpm}$} & \multicolumn{2}{|c|}{$8000 \mathrm{rpm}$} & \multicolumn{2}{|c|}{$10 \mathrm{rpm}$} & \multicolumn{2}{|c|}{$8000 \mathrm{rpm}$} & \multicolumn{2}{|c|}{$10 \mathrm{rpm}$} & \multicolumn{2}{|c|}{$8000 \mathrm{rpm}$} \\
\hline $\begin{array}{l}\text { Freq. } \\
(\mathrm{Hz})\end{array}$ & $\begin{array}{l}\text { Damp } \\
\text { Ratio }\end{array}$ & $\begin{array}{l}\text { Freq. } \\
\text { (Hz) }\end{array}$ & $\begin{array}{l}\text { Damp } \\
\text { Ratio }\end{array}$ & $\begin{array}{c}\text { Freq. } \\
(\mathrm{Hz})\end{array}$ & $\begin{array}{l}\text { Damp } \\
\text { Ratio }\end{array}$ & $\begin{array}{l}\text { Freq. } \\
(\mathrm{Hz})\end{array}$ & $\begin{array}{l}\text { Damp } \\
\text { Ratio }\end{array}$ & $\begin{array}{l}\text { Freq. } \\
(\mathrm{Hz})\end{array}$ & $\begin{array}{l}\text { Damp } \\
\text { Ratio }\end{array}$ & $\begin{array}{l}\text { Freq. } \\
(\mathrm{Hz})\end{array}$ & $\begin{array}{l}\text { Damp } \\
\text { Ratio }\end{array}$ \\
\hline 24.9 & 0.702 & 22.3 & 0.702 & 24.7 & 0.716 & 22.2 & 0.724 & 24.7 & 0.716 & 22.3 & 0.729 \\
\hline 24.9 & 0.702 & 27.9 & 0.690 & 24.7 & 0.716 & 27.6 & 0.698 & 24.7 & 0.716 & 27.7 & 0.689 \\
\hline 53.5 & 0.597 & 52.7 & 0.590 & 52.9 & 0.617 & 51.7 & 0.621 & 52.9 & 0.617 & 51.7 & 0.626 \\
\hline 53.5 & 0.597 & 54.8 & 0.605 & 52.9 & 0.617 & 54.2 & 0.617 & 52.9 & 0.617 & 54.2 & 0.614 \\
\hline 138.1 & 0.036 & 130.9 & 0.039 & 138.8 & 0.053 & 131.8 & 0.060 & 138.8 & 0.053 & 131.9 & 0.062 \\
\hline 138.1 & 0.036 & 145.5 & 0.032 & 138.8 & 0.053 & 146.1 & 0.047 & 138.8 & 0.053 & 146.0 & 0.045 \\
\hline 353.2 & 0.033 & 340.5 & 0.036 & 352.7 & 0.039 & 340.3 & 0.042 & 352.7 & 0.039 & 340.3 & 0.042 \\
\hline 353.3 & 0.033 & 365.3 & 0.031 & 352.7 & 0.039 & 364.6 & 0.036 & 352.7 & 0.039 & 364.6 & 0.035 \\
\hline 632.2 & -0.026 & 600.7 & -0.020 & 634.0 & -0.024 & 603.1 & -0.018 & 634.0 & -0.024 & 603.1 & -0.018 \\
\hline 632.3 & -0.026 & 663.3 & -0.032 & 634.1 & -0.024 & 664.5 & -0.029 & 634.1 & -0.024 & 664.5 & -0.030 \\
\hline
\end{tabular}

The root locus plots for each case are displayed below. The operating speeds range from 10 to $8000 \mathrm{rpm}$.

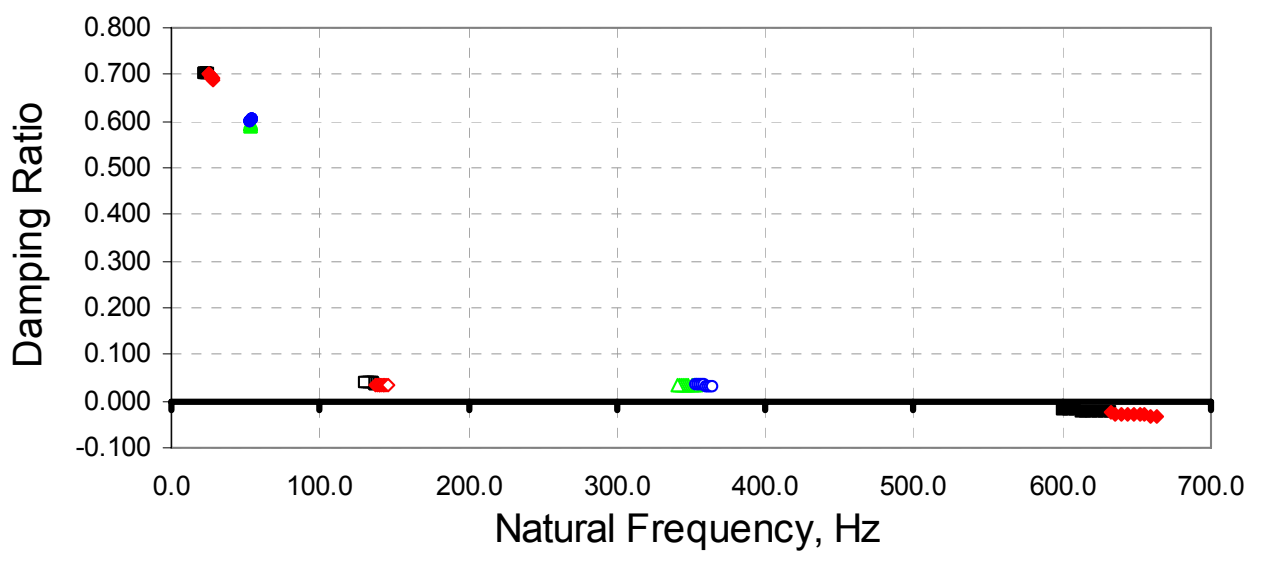

Fig. 102 Rotordynamic root locus plots for no seal configuration 


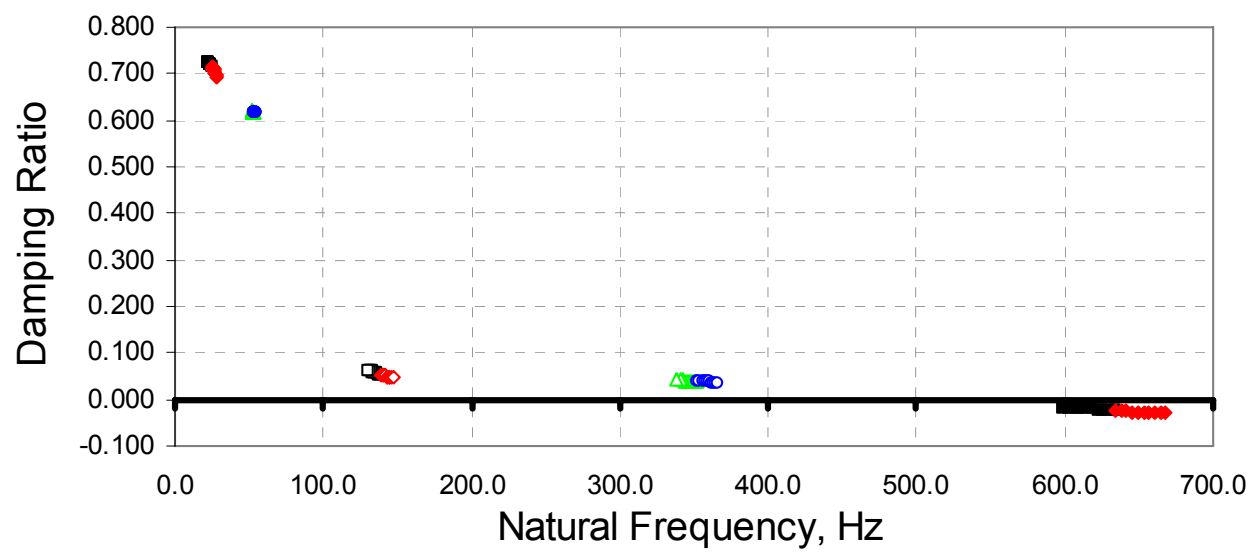

Fig. 103 Rotordynamic root locus plots for seals without preswirl configuration

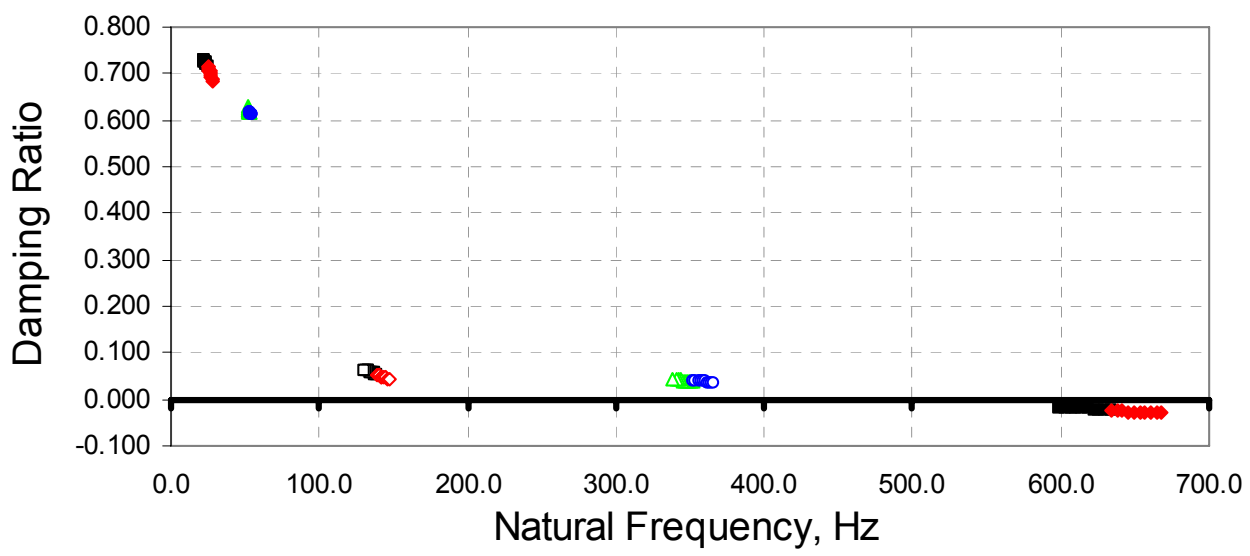

Fig. 104 Rotordynamic root locus plots for seals with preswirl configuration

The final consideration of this analysis is the imbalance response at the seal locations. An imbalance of $36 \mathrm{~kg}-\mu \mathrm{m}(0.05 \mathrm{oz}-\mathrm{in})$ was applied to the rotor at the center location. This imbalance was selected because it is an estimate of the possible imbalance based the balance data of rotor after the most recent servicing. The plot below displays the response of the rotor at the center. 


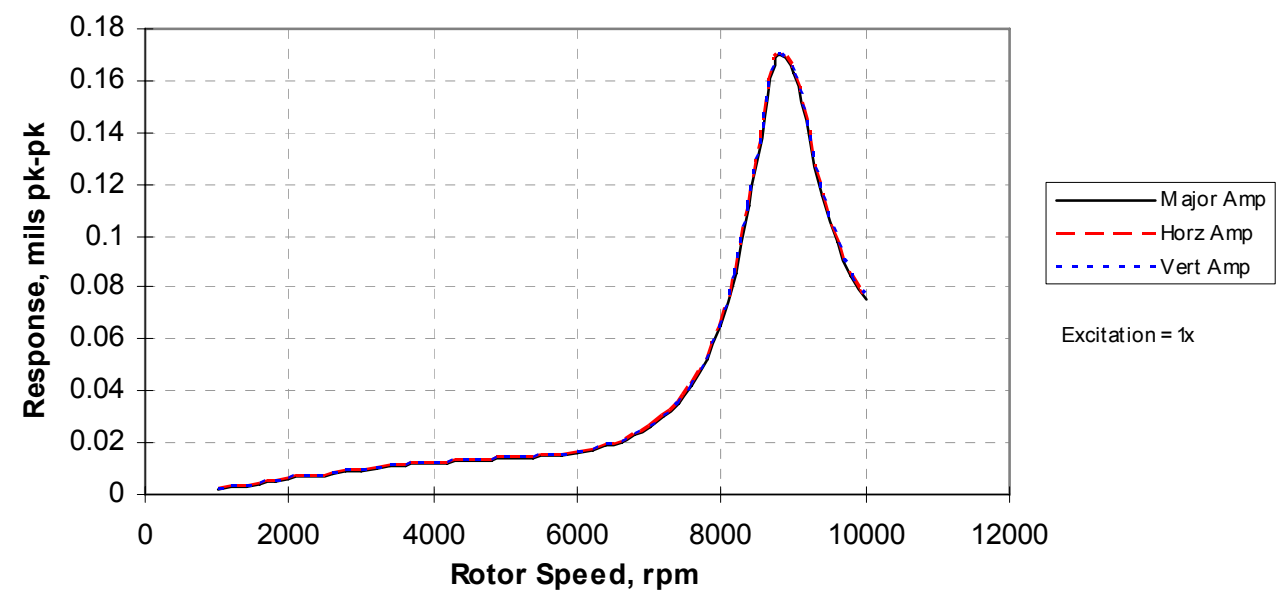

Fig. 105 Imbalance response at rotor center

The following plot is the deflected shape of the rotor at the critical speed $(8800 \mathrm{rpm})$. Note that the maximum achievable rotor speed with the current test rig configuration is $8000 \mathrm{rpm}$.

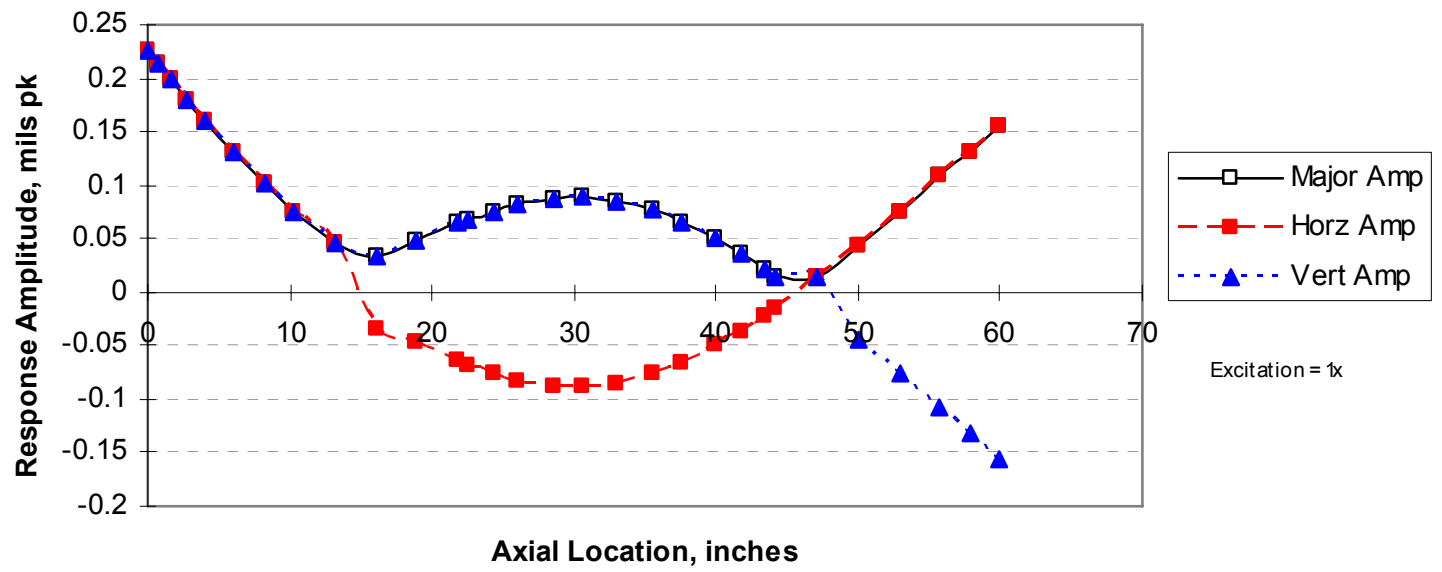

Fig. 106 Deflected shape at the critical speed 


\section{Conclusions}

Neither the seal models nor the rotordynamic analysis of the test rig have produced definitive results as to the exact effects of adding seals to the test rig; however, by comparing results, general conclusions can be surmised.

The leakage predictions for the seals are confirmed by comparison with a leakage equation calculation for a single-tooth labyrinth. The leakage and pressure are therefore considered reasonable predictions. The exit mach number for the smooth seals is 0.2 , indicating that the labyrinth seals fulfill their primary purpose of preventing choked flow in the smooth seals.

Some of the eigenvalues (above $600 \mathrm{~Hz}$ ) of the test rig models contain positive real parts, indicating instability. These values are present in the model for the current setup, which is known to have stable operation. The explanation for these values is unclear. It can be seen from comparing the results with and without seals that the addition of the seals slightly improves the stability over the operating range.

The response plots show a maximum peak-to-peak vibration of $4.3 \mu \mathrm{m}(0.00017 \mathrm{in}$.) at the seals. This is less than $1 \%$ of the seal clearance, and it is therefore not problematic.

To determine the onset speed of instability, the analysis with no preswirl was repeated for higher operating speeds. The root locus plot is shown below. The first transition to a negative damping ratio for frequencies below $600 \mathrm{~Hz}$ occurs at 140,000 rpm. The results also show that the damping ratios do not begin to decrease substantially until $25,000 \mathrm{rpm}$. These results indicate that excessive imbalance response and instability caused by the addition of the seals are not risks for the current operating speed range. 


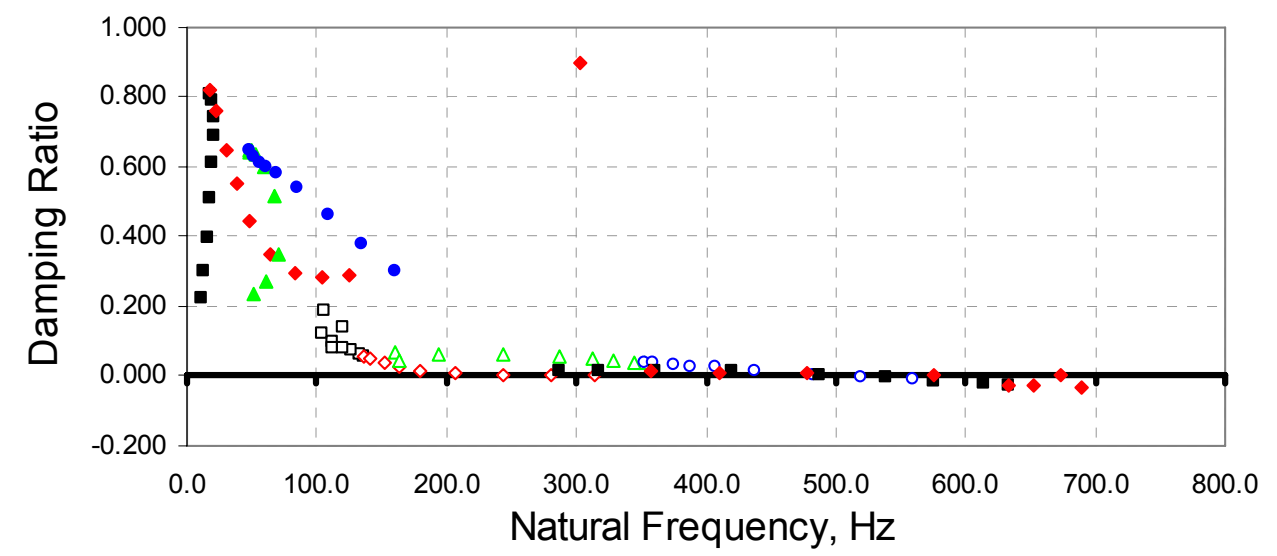

Fig. 107 Rotordynamic root locus plot for seals without preswirl configuration 


\section{VITA}

Name:

Address:

Email Address:

Education:
Zachary Scott Zutavern

7960 Briaridge Rd.

Dallas, TX 75248

lzzutavern@sbcglobal.net

B.S., Mechanical Engineering, Texas A\&M University, 2002

B.A., Physics, Texas A\&M University, 2002

M.S., Mechanical Engineering, Texas A\&M University, 2004

Ph.D., Mechanical Engineering Texas A\&M University, 2006 